\section{ASTHMA \& ALLERGY SIG: POSTER SESSION 1}

MULTIFREQUENCY SIGNALS IDENTIFY EXPIRATORY FLOW LIMITATION USING FORCED OSCILLATION TECHNIQUE

\section{S MAHADEV ${ }^{1,2,3}, \mathrm{CHTUN}^{1}$, CM SALOME ${ }^{2,4}, \mathrm{GG} \mathrm{KING}^{1,2,3,4}$,} R SCHOEFFEL 2,3,4

${ }^{1}$ Department of Respiratory Medicine, Royal North Shore Hospital, St Leonards, NSW 2065, ${ }^{2}$ Woolcock Institute of Medical Research, Glebe NSW 2037, ${ }^{3}$ Northern Clinical School, University of Sydney, NSW 2006, and ${ }^{4}$ CRC for Asthma and Airways, Glebe 2037

Expiratory flow limitation (EFL) during tidal breathing is associated with chronic exertional dyspnoea in COPD. Respiratory system reactance (Xrs) measured by the forced oscillation technique (FOT) can be used to detect EFL with $100 \%$ sensitivity and $100 \%$ specificity against gold standard measures (Dellaca et al. ERJ 2004). The validation study used a $5 \mathrm{~Hz}$ monofrequency oscillation signal. It is unknown whether multifrequency signals are comparable to $5 \mathrm{~Hz}$ in detecting EFL.

Aim To determine if FOT multifrequency signals of $6,11,19 \mathrm{~Hz}$ and 5,11 , $19 \mathrm{~Hz}$ are comparable to $5 \mathrm{~Hz}$ in the detection of EFL in COPD patients.

Methods 25 subjects with greater than 10 pack years smoking history and a clinical history of COPD underwent FOT during 60 seconds of tidal breathing, using $5 \mathrm{~Hz}, 5,11,19 \mathrm{~Hz}$, and $6,11,19 \mathrm{~Hz}$ oscillation signals in random order. The difference between mean inspiratory and expiratory respiratory system reactance $(\Delta \mathrm{Xrs})$ was calculated. Subjects had $E F L$ if the mean $\Delta \mathrm{Xrs}$ was $\geq 2.8 \mathrm{~cm} \mathrm{H} 2 \mathrm{O} . \mathrm{s} . \mathrm{I}^{-1}$ at $5 \mathrm{~Hz}$

Results Mean FEV $\%$ predicted was 76 (range: $31-125) 7$ subjects had EFL on $5 \mathrm{~Hz}$, and of these 6 subjects had EFL on $5,11,19 \mathrm{~Hz}$, and 6 subjects had $\mathrm{EFL}$ on $6,11,19 \mathrm{~Hz}$. Five subjects had EFL on all 3 frequencies tested. Both multifrequency signals had sensitivity $85 \%$ and specificity of $94 \%$ compared to $5 \mathrm{~Hz}$. There was no significant difference in $\Delta \mathrm{Xrs}$ between the three oscillation signals (ANOVA $p=0.18$ ).

Conclusions There was a high degree of concordance between the three oscillation signals used to assess EFL in this study. This study supports the use of multifrequency oscillation signals for the detection of EFL.

Support S Mahadev - Asthma Foundation NSW stipend.

Conflict of Interest No.

\section{UTILITY OF MANNITOL AS CHALLENGE TEST FOR ASTHMA AND EXERCISE-INDUCED ASTHMA IN CHILDREN}

\author{
E AL-ZAYADNEH, B KENNEDY, A MERCHANT, P VAN ASPEREN, \\ H SELVADORI \\ Department of Respiratory Medicine, Children's Hospital at Westmead, \\ NSW, Australia
}

Mannitol has been increasingly used as a challenge test for asthma and exercise-induced asthma in children. As an osmotic agent, it provides an objective measure of airway hyperreactivity.

Aim To assess the frequency of positive mannitol tests in children who were referred with a history of asthma symptoms.

Method Retrospective chart review of children aged 6 to 18 years who were referred for a mannitol challenge tests in the past 2 years with a history of 'asthma symptoms'. 'Asthma symptoms' was defined as parent reported wheeze or shortness of breath on exercise suggestive of EIA.

Results We identified a total of 80 patients who performed mannitol tests. Eleven patients had positive test $(13.7 \%)$. Of these, seven $(63.6 \%)$ had an atopic background and four $(36.3 \%)$ were already being treated with inhaled corticosteroids prior to the test. Of the 69 children with negative mannitol tests, $20(29 \%)$ were being treated with inhaled corticosteroids and $15(21.7 \%)$ were atopic. Mean predicted $\mathrm{FEV}_{1}$ was $(108.1, \pm 16 \mathrm{SD})$, mean percentage of $\mathrm{FEV}_{1}$ drop $(14.5, \pm 3.5 \mathrm{SD})$. Twenty of the patients with negative mannitol tests had exercise tests. The exercise tests demonstrated reduced physical fitness as the cause of reduced exercise ability.

Conclusion This chart review suggests that a history of asthma symptoms does not equate with a positive mannitol challenge test. There are other causes of 'asthma symptoms' that can be defined further with exercise testing. Further data collection and statistical analysis are in process.

Conflict of Interest No.

\section{COMPARISON OF MANNITOL AND METHACHOLINE INHALATION CHALLENGES IN SUBJECTS WITH OCCUPATIONAL ASTHMA}

C LEMIERE, D MIEDINGER, V JACOB, S CHABOILLEZ, C TREMBLAY Hôpital du Sacré-Coeur de Montréal, University of Montreal, Canada

Objective (1) To compare the airway responsiveness assessed using a mannitol inhalation challenge (MAN) and a methacholine inhalation challenge (METH) and to evaluate their respective correlation with airway inflammation in subjects with previously diagnosed occupational asthma (OA). (2) To compare the sputum cell counts and the quality of the sputum sample obtained after inhalation of hypertonic saline and after MAN.

Methods Subjects completed a MAN and a METH 2-7 days apart, in a random order. A sputum sample was obtained after inhalation of hypertonic saline following the METH or during the MAN. MAN was considered positive if the cumulative dose of mannitol inducing a $15 \%$ fall in FEV (PD15) was $^{2}$ $\leq 635 \mathrm{mg}$; a METH was considered positive if the dose of methacholine inducing a $20 \%$ fall in $\mathrm{FEV}_{1}\left(\mathrm{PC}_{20}\right)$ was $\leq 16 \mathrm{mg} / \mathrm{mL}$. Airway responsiveness was also expressed as the dose response slope of METH (DRSMETH) or MAN (DRSMAN) for all subjects.

Results Twenty-two subjects with OA were enrolled. METH identified airway hyperresponsiveness in $68.2 \%$ of cases, MAN in $40.9 \%$ of cases; $p=0.01$. DRSMETH and DRSMAN were significantly correlated $(r=0.7 p<0.001)$. Sputum eosinophils were correlated with DRS MAN (rho $=0.5 ; p=0.009$ ), but not with DRSMETH (rho $=0.2, p=0.5$ ). The quality of the sputum samples obtained after hypertonic saline or MAN was similar.

Conclusions MAN may represent an interesting alternative to METH in measuring airway responsiveness and inducing sputum during the same procedure.

Supported by Asthma in the Workplace Centre (CIHR).

Nomination N/A.

Conflict of Interest No.

Journal Compilation @ 2011 Asian Pacific Society of Respirology

\title{
(3)
}


RELIABILITY AND VALIDITY OF THE SMARTRACK@ DEVICE: A NEW ELECTRONIC MONITOR FOR METERED DOSE INHALERS

JM FOSTER ${ }^{1}$, L SMITH ${ }^{2}$, T USHERWOOD ${ }^{3}$, SM SAWYER ${ }^{4}$, HK REDDEL ${ }^{1}$ ${ }^{1}$ Woolcock Institute of Medical Research, NSW, Australia, ${ }^{2}$ University of Sydney, NSW, Australia, ${ }^{3}$ Sydney Medical School, NSW, Australia, and ${ }^{4}$ Centre for Adolescent Health, Vic., Australia

Background The SmartTrack® (Nexus6, Auckland, NZ) is a new reusable monitoring device with remote upload capability and ringtone reminders for assessing patient usage of pressurized metered dose inhalers. Our aim was to assess its reliability and validity.

Methods Study 1 Bench-test: 10 salmeterol/fluticasone inhalers fitted with SmartTrack $@$ devices were actuated by one researcher two times twice daily for 48 hours. Accuracy of reminder ringtones, data logging and data upload was tested. Study 2 Field-test: Devices were quality control tested prior to dispensing. Asthma patients each field-tested one SmartTrack $\circledast$ for 7 days and recorded all actuations in a paper diary. Data were automatically uploaded by SIM card and compared with patient diaries. Patient-reported ease of use for the device was recorded.

Results Study 1: Nine of 10 devices passed bench-testing. One device failed bench-testing due to failure to log any actuation. Study 2: One device failed pre-dispensing quality control testing (electrical circuit failure); thus, 8 patients (mean age 45 years, 5 females, $\mathrm{FEV}_{1} \%$ pred. $84.1 \pm 23$ ) field-tested the remaining devices. All devices passed field testing, apart from a minor error in two devices (actuation logged during pMDI insertion). Six devices had $100 \%$ accurate actuation logging, and mean accuracy for the 8 devices was $92 \%$. All devices successfully uploaded the inhaler usage data. Patients reported that it was easy to use the device.

Discussion The SmartTrack® has acceptable validity and reliability comparable to that of currently available adherence monitoring devices. The advantages of the device include a customizable ringtone reminder function to enhance or maintain adherence, and remote data uploading which may reduce data loss.

Supported by NHMRC, GlaxoSmithKline (medications).

Nomination None.

Conflict of Interest Yes.

\section{INTERACTIONS BETWEEN TOBACCO SMOKE AND GST GENETIC VARIANTS ON LUNG FUNCTION AND RESPIRATORY SYMPTOMS IN ADULTS FROM GREENLAND}

CM HAYDEN ${ }^{1}$, S CHEOW ${ }^{1}$, J MURDZOSKA ${ }^{1}$, EN SCHULTZ ${ }^{1}$, SK KHOO', G ZHANG ${ }^{1}$, V BACKER ${ }^{2}$, J GOLDBLATT ${ }^{1}$, PN LE SOUËF ${ }^{1}$,

SG DEVADASON ${ }^{1}$

${ }^{1}$ School of Paediatrics and Child Health, University of Western Australia, WA, Australia, and ${ }^{2}$ Department of Internal Medicine, Bispebjerg University Hospital Copenhagen, Denmark

Aim To investigate if tobacco smoke exposure and variants in the GSTP1 (ile105val), GSTM1 (null/non-null) and GSTT1 (null/non-null) genes influence lung function and asthmatic phenotypes in 433 adults living in Nuuk, Greenland.

Methods Genotyping for the 3 GST genes was done on 433 individuals by polymerase chain reaction. Cotinine measurements were done on 244 urine samples to validate questionnaire data on tobacco smoke exposure. Information on asthma and respiratory symptoms was recorded in questionnaires and lung function was measured using spirometry.

Results Genotype frequencies were similar to those previously reported and the cotinine measurements validated the questionnaire data on tobacco smoke exposure. Significant associations were found between the GSTP1 105 ile variant and between a combination of GSTP1 105 ile and GSTM1 null and reduced lung function $(p=0.040$ and $p=0.027)$. Significant associations were found between higher levels of tobacco smoke exposure and both reduced lung function and bronchitis $(p=0.039$ and $p=0.003)$. There were no significant interactions between tobacco smoke exposure and the GST genetic variants studied on lung function and asthmatic phenotypes.

Conclusion Tobacco smoke exposure and the selected GST genetic variants did not have an interactive effect on respiratory health outcomes in the study population. Other GST genes or detoxification pathways may play a more significant role in the study cohort.

Conflict of Interest Nil.

\section{CHARACTERIZING THE SEVERE ASTHMA PHENOTYPE IN AN} AUSTRALIAN SETTING: THE AMAZES STUDY

CG GARSIDE ${ }^{1,2}$, JL SIMPSON ${ }^{1,2}$, IA YANG $^{3}$, AL JAMES $^{4}, \mathrm{~J} \mathrm{UPHAM}^{5}$, PN REYNOLDS ${ }^{6}$, SJ HODGE ${ }^{6}, P^{\prime}$ GIBSON ${ }^{1,2}$, AMAZES STUDY RESEARCH GROUP

${ }^{1}$ Centre for Asthma and Respiratory Disease, The University of Newcastle, NSW, ${ }^{2}$ Department of Respiratory and Sleep Medicine, Hunter Medical Research Institute, NSW, ${ }^{3}$ The Prince Charles Hospital and ${ }^{5}$ Princess Alexandra Hospitals, School of Medicine, The University of Queensland, Brisbane, Qld, ${ }^{4}$ Sir Charles Gairdner Hospital, Perth, WA, and ${ }^{6}$ Department of Thoracic Medicine, Royal Adelaide Hospital and Hanson Institute, Adelaide, $S A$

Introduction Severe asthma leads to an imposing health-care burden and may exhibit significant phenotypic heterogeneity that can influence treatment responsiveness. Severe asthma phenotypes have been described by European and American consortia; however, Australian data are limited. The aim of this study is to characterize the phenotypes of severe asthma in Australia, using clinical, physiological and inflammatory characteristics.

Methods Non-smoking adults $(n=111)$ were assessed for the AMAZES study, a multicentre RCT evaluating add-on azithromycin in severe asthma, in 5 tertiary centres across Australia. Subjects had asthma and poor disease control $(A C Q>0.75)$ despite maintenance ICS/LABA. They underwent clinical assessment, skin allergy test, hypertonic saline challenge and induced sputum cell counts.

Results Subjects with severe asthma had a mean age of 57 years, with high levels of atopy $(85 \%)$ and mild airflow obstruction ( $\mathrm{FEV}_{1} \sim 70 \%$ pred.). There was a high prevalence of co-morbidity and $12 \%$ reported aspirin intolerance. Average BMI was $31 \mathrm{~kg} / \mathrm{m}^{2}$ and $47 \%$ reported working in a dusty job. The inflammatory phenotype was classified as eosinophilic in $44 \%$, neutrophilic in $14 \%$, paucigranulocytic in $30 \%$ and mixed eosinophil/neutrophil in $12 \%$. There was variation across Australia in atopy prevalence, medication use (theophylline), and degree of sputum neutrophilia.

Conclusion Severe asthma in Australia exhibits significant phenotypic heterogeneity, particularly in the airway inflammatory response. The Australian population with severe asthma differs from overseas cohorts with a higher prevalence of overweight, atopy and aspirin intolerance.

Supported by NHMRC.

Conflict of Interest No.
Support ACAM is a collaborating unit of the AlHW and funded by the Australian Government Department of Health and Ageing.

Conflict of Interest No. 
TP-009

\section{GALECTIN-10, A POTENTIAL BIOMARKER OF EOSINOPHILIC} AIRWAY INFLAMMATION IN ASTHMA

\author{
JC CHUA ${ }^{1,2}$, JA DOUGLASS $^{1}$, A GILLMAN ${ }^{1}$, K SYMONS $^{1}$, K DECKERT $^{1}$, \\ RE O'HEHIR ${ }^{1}$, E MEEUSEN ${ }^{2}$ \\ ${ }^{1}$ Department of Allergy, Immunology and Respiratory Medicine, Alfred \\ Hospital \& Monash University, Melbourne, Vic., Australia, ${ }^{2}$ School of \\ Biomedical Sciences, Monash University, Clayton, Vic., Australia
}

Aim To assess the relationship between galectin-10 in sputum with sputum eosinophil measurements and other clinical asthma parameters in asthma. Methods Participants completed an Asthma Control Questionnaire (ACQ), performed spirometry and produced a sputum sample either spontaneously or by sputum induction. Thirty-eight sputum samples were collected: 27 asthmatic, 7 healthy control, 3 bronchiectasis and 1 interstitial pulmonary fibrosis. Sputum eosinophil percentages (of leukocytes counted) and numbers per gram of sputum were measured in each sample. Sputum supernatants were tested for galectin-10 by Western blot and image analysis, semi-quantified against a standard sample and expressed as relative units.

Results Sputum galectin-10 amounts had a strong correlation with sputum eosinophil percentages $(0.695, p<0.001)$, and an even stronger correlation with sputum eosinophil numbers $(0.786, p<0.001)$, but no relationship with sputum neutrophil percentages $(p=0.141)$. Sputum galectin-10 was able to determine sputum eosinophil percentage of $>3 \%$ with a sensitivity of $100 \%$ and a specificity of $88.9 \%$, and had an area under the receiver operating characteristic (ROC) curve of 0.963 . The amount of galectin-10 in sputum had a moderate correlation with both the ACQ $(0.411, p=0.019)$ and the prebronchodilator $\mathrm{FEV}_{1}$ percentage predicted $(-0.406, \mathrm{p}=0.021)$, while sputum eosinophil percentages did not ( $p=0.230$ and 0.379 , respectively).

Conclusion Galectin-10 in sputum has a strong association with sputum eosinophil measurements, providing validation that galectin-10 could constitute an alternative measure of eosinophilic airway inflammation. Conflict of Interest None declared.

\section{GRANZYME B IS INCREASED IN INDUCED SPUTUM BOTH FROM PATIENTS WITH COPD AND NON-EOSINOPHILIC ASTHMA}

S HODGE ${ }^{1,2}, \mathrm{~S}_{\text {RICHARDS }}^{1,2}, \mathrm{JL} \mathrm{SIMPSON}^{3,4}, \mathrm{C}$ GARSIDE $^{3,4}$

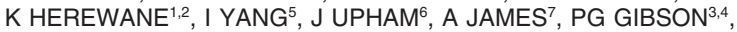
PN REYNOLDS ${ }^{1,2}$; AMAZES STUDY RESEARCH GROUP ${ }^{1}$ Thoracic Medicine, Royal Adelaide Hospital, ${ }^{2}$ Lung Research, Hanson Institute, Adelaide, ${ }^{3}$ Centre for Asthma and Respiratory Disease, University of Newcastle, ${ }^{4}$ Department of Respiratory and Sleep Medicine, Hunter Medical Research Institute, ${ }^{5}$ The Prince Charles Hospital and ${ }^{6}$ Princess Alexandra Hospitals, Brisbane, School of Medicine, University of Queensland, and ${ }^{7}$ WA Sleep Disorders Research Institute, Charles Gairdner Hospital, Perth

CD8+ T-cells may cause airway epithelial cell apoptosis via the granzyme pathway. We have reported increased apoptosis of airway epithelial cells and increased BAL T-cell expression of granzyme B in COPD, with a positive correlation between the two $0^{1,2}$. We hypothesized that increased granzyme $B$ would also be detected in induced sputum from COPD patients, and that a similar increase would also be evident in sputum from patients with the non-eosinophilic form of asthma (NEA).

Methods We investigated T-cell intracellular granzyme $B$ and its inhibitor PI-9, in sputum from control subjects $(n=9)$, and patients with COPD $(n=4)$, NEA $(n=6)$ or eosinophilic asthma (EA) $(n=7)$ and mixed EA/NEA $(n=8)$, using flow cytometry.

Results Significantly increased granzyme $B$ was found in sputum from COPD subjects $26.1 \%(9.2)$ (mean (SEM)) versus control $3.8 \%(1.8), p=0.02$, findings consistent with those previously seen in BAL: COPD $33.6 \%(4.8)$ versus control $8.0 \%(1.3), p=0.02$. Granzyme $B$ was also increased in patients with NEA $(28.0 \%(9.9))$ or EA/NEA (22.0 (9.9)) versus control (3.8\% (1.8)), p $=0.043$ and 0.049 , respectively. There were no significant changes in granzyme B in EA or in PI-9 in any patient group.

Conclusions Induced sputum provides a non-invasive tool for investigating T-cell cytotoxic mediators in the various asthma sub-types. Increased expression of granzyme B by airway T-cells may contribute to increased epithelial cell apoptosis, lung injury and chronic inflammation in NEA.

References

1 Hodge S. Eur Respir J 2005

2 Hodge S. J COPD 2006

Support NHMRC.

Conflict of Interest Nil.

\section{NON-EOSINOPHILIC ASTHMA RESEMBLES COPD WITH SUPPRESSED APOPTOTIC CELL CLEARANCE AND MACROPHAGE RECOGNITION MOLECULES}

S HODGE ${ }^{1,2}$, JL SIMPSON ${ }^{3,4}$, S RICHARDS ${ }^{1,2}$, C GARSIDE ${ }^{3,4}$, I YANG ${ }^{5}$, J UPHAM ${ }^{6}$, A JAMES ${ }^{7}$, PG GIBSON ${ }^{3,4}$, PN REYNOLDS ${ }^{1,2}$, AMAZES STUDY RESEARCH GROUP

${ }^{1}$ Thoracic Medicine, Royal Adelaide Hospital, ${ }^{2}$ Lung Research Laboratory, Hanson Institute, Adelaide, SA, ${ }^{3}$ Centre for Asthma and Respiratory Disease, University of Newcastle, ${ }^{4}$ Department of Respiratory and Sleep Medicine, Hunter Medical Research Institute, NSW, ${ }^{5}$ The Prince Charles Hospital and ${ }^{6}$ Princess Alexandra Hospitals, Brisbane, School of Medicine, University of Queensland, and ${ }^{7}$ WA Sleep Disorders Research Institute, Charles Gairdner Hospital, Perth, WA

Patients with non-eosinophilic asthma (NEA) or COPD have increased numbers of neutrophils in the airways. We have shown a similar defect in the ability of alveolar macrophages (AM) to phagocytose apoptotic cells, in sputum from patients with NEA and COPD. We have also shown that BAL-derived AM from patients with COPD have reduced expression of key macrophage phagocytic recognition molecules. The aim of this pilot study was to investigate the expression of these macrophage markers in induced sputum from patients with eosinophilic asthma (EA, $n=10)$, NEA $(n=13)$, COPD $(n=7)$ and controls $(\mathrm{n}=5)$.

Methods Participants underwent clinical assessment, skin allergy test, hypertonic saline challenge and sputum induction. Macrophage phagocytosis of apoptotic cells, expression of mannose receptor (MR), HSPR (CD91) and PCAM (CD31) was determined using flow cytometry.

Results Phagocytosis was significantly impaired in patients with NEA and COPD. Expression of MR, CD91 and CD31 were decreased in patients with NEA or COPD, but not significantly changed in EA

Conclusion Impaired sputum-macrophage phagocytosis of apoptotic cells in NEA is associated with reduced expression of key macrophage recognition molecules. This defect may contribute to the chronic inflammation and persistent airway neutrophilia that characterizes this asthma subtype. The use of induced sputum as a surrogate for the more-invasive bronchoscopic sampling may provide a tool for investigating the mechanisms for the effect of therapies including azithromycin in lung disease.

Supported by NHMRC
TP-012

\section{THE ASTHMA SNAPSHOT: NATIONAL ASTHMA STATISTICS ONLINE}

MR GALL, AL WEBSTER

Australian Institute of Health and Welfare, Canberra, ACT

The Asthma Snapshot, an online resource, has been developed to provide the latest available national summary statistics regarding asthma, a complement to more detailed AlHW reports.

Methods The Asthma Snapshot includes statistics from the:

- Australian Bureau of Statistics National Health Survey;

- Bettering the Evaluation And Care of Health (BEACH) Survey of General

Practice;

- Pharmaceutical Benefits Scheme;

- IMS Health pharmaceutical data;

- AlHW National Hospital Morbidity Database;

- AlHW National Mortality Database; and the

- AlHW Disease Expenditure Database.

Results The Asthma Snapshot provides the latest national statistics related to commonly asked questions such as:

- Who gets asthma?

- What role do GPs play in treating asthma?

- What role do hospitals play in treating asthma?

- How does asthma affect quality of life?

- How many die from asthma?

- What is the health burden of asthma?

- How much is spent on asthma?

Conclusions The Asthma Snapshot allows quick access to a range of important summary statistics regarding asthma.

Supported by Australian Government Department of Health and Ageing. Conflict of Interest No.

Journal Compilation (C) 2011 Asian Pacific Society of Respirology 
ASTHMA \& ALLERGY SIG: POSTER SESSION 2

TP-013

\section{TRANSFORMING GROWTH FACTOR BETA SYNERGIZES WITH KININS IN INCREASING HUMAN DENDRITIC CELLS MIGRATION}

\section{FOGEL-PETROVIC, NL MISSO, CM BERTRAM, S BALTIC,} PJ THOMPSON

Lung Institute of Western Australia, Centre for Asthma, Allergy \& Respiratory Research, University of Western Australia, Nedlands, Australia

Aim Within the airways, epithelial cells and fibroblasts are significant sources of transforming growth factor $\left(\operatorname{TGF} \beta_{1}\right)$. TGF $\beta_{1}$ induces chemotaxis and the expression of proteins necessary for cell migration. Since increased migration has been associated with development of asthma-like features, we assessed effect of TGF $\beta_{1}$ on dendritic cells (DC) migration and on expression of migration-related kinin receptors and compared migration of DC from asthmatic and healthy patients.

Methods Human monocyte-derived dendritic cells (hMo-DC) were stimulated with TGF $\beta_{1}$ and cell migration was assessed using Trans-well chambers. Expression of kinin receptors was assessed by RT-PCR and flow-cytometry or immunolabeling.

Results TGF $\beta_{1}$ alone acts as chemoatractant for immature hMo-DC but also synergizes with Bradykinin (BK) to increase cell migration. TGF $\beta_{1}$ potentiates effect of Lys-des-Arg ${ }^{9}$-BK (Lda-BK), which decreases CCL19-directed migration of mature hMo-DC. Mo-DC stimulated with TGF $\beta_{1}$ show an increase in the expression of both kinin receptors in a concentration-dependent manner. Kinin receptor expression as well as BK, Lda-BK and CCL5-directed migration is increased in hMo-DC from asthmatics subjects, while CCL19-directed migration is decreased, compared to healthy subjects.

Conclusions In homeostasis, TGF $\beta_{1}$ and BK (released in the lung by a variety of cell types) may cooperate to enhance migration of immature DC. In the inflamed lung tissues (asthma patients), increased levels of BK and TGF $\beta_{1}$ maintained the recruitment of immature DC, while increased formation of Lda-BK decrease mature DC migration, 'trapping' them in the inflamed tissue. These data suggest that TGF $\beta_{1}$ and kinins may cooperate to increase the accumulation of DC that has been observed in the lungs of asthmatic patients. Conflict of Interest No.

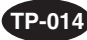

\section{LACK OF EVIDENCE FOR NEUTROPHIL ACTIVATION IN NON- EOSINOPHILIC ASTHMA}

\section{CR BROOKS ${ }^{1}$, CJ VAN DALEN ${ }^{1}$, EE HARDING ${ }^{1}$, IF HERMANS ${ }^{2}$, J DOUWES \\ ${ }^{1}$ Centre for Public Health Research, Massey University, Wellington, New Zealand, and ${ }^{2}$ Malaghan Institute of Medical Research, Wellington, New Zealand}

Aim To investigate sputum neutrophil activation in non-eosinophilic asthma Methods Participants were recruited by advertisement in medical practices and by invitation. Each participant completed an asthma questionnaire and underwent spirometry followed by hypertonic saline $(4.5 \%)$ sputum induction. Sputum samples were processed using standardized methods. Asthma inflammatory phenotype was defined by sputum eosinophil percentage, i.e. noneosinophilic asthma - sputum eosinophils $<2 \%$. Cells were analysed by flow cytometry by staining with fluorescently labelled antibodies specific for CD 45 , CD14, CD16 and CD11b (or relevant isotype control). Supernatant was analysed for myeloperoxidase (MPO), IL-8, MMP-9 and MMP-9/TIMP-1 with commercially available ELISA kits.

Results 54 subjects were recruited and $45(83 \%, M / F=21 / 24$, age 29.5 , 17-62 (years, median, range)) produced a satisfactory sputum sample. Three subjects were excluded (past history of asthma in a control subject (1), current smoking (2)). Twenty-four subjects had asthma (8 eosinophilic (EA), 16 noneosinophilic (NEA)), 18 were healthy controls. There was no statistically significant difference in percentage of sputum neutrophils, neutrophil CD11b expression, levels of sputum supernatant MPO, IL-8, MMP-9 or MMP-9/ TIMP-1, between EA, NEA and control subjects.

Conclusions These results suggest that the neutrophil, while present in sputum, is not differently activated in NEA compared with either EA or control subjects. This brings into question the importance of the neutrophil in contributing to the immunopathogenesis of non-eosinophilic asthma. Supported by Wellington Medical Research Foundation. Conflict of Interest No.

Journal Compilation (C) 2011 Asian Pacific Society of Respirology

\section{IS CHLAMYDOPHYLA PNEUMONIAE INFECTION RELATED TO} NON-EOSINOPHILIC ASTHMA? F WANG ${ }^{1,2,3}, X Y \mathrm{HE}^{2,3}, \mathrm{KJ}_{\text {BAINES }}^{2,3}$, LP GUNAWARDHANA $^{2,3}$,
JL SIMPSON $^{2,3}, \mathrm{~F} \mathrm{LI}^{1}$, PG GIBSON

${ }^{1}$ Department of Pathogenobiology, Norman Bethune College of Medicine, Jilin University, Changchun, China, ${ }^{2}$ Centre for Asthma and Respiratory Disease, University of Newcastle, Callaghan, NSW, Australia, and ${ }^{3}$ Department of Respiratory and Sleep Medicine, Hunter Medical Research Institute, John Hunter Hospital, New Lambton, NSW, Australia

Aim The aim of this study was to examine for an association between inflammatory phenotype and Chlamydophyla pneumoniae infection in adults and children with stable and acute asthma.

Methods Adults with stable asthma $(n=29)$, acute asthma $(n=22)$, healthy adults $(n=11)$, and children with stable asthma $(n=49)$, acute asthma $(n=$ 28 ) and healthy children $(n=9)$ had undergone clinical assessment. Sputum was assessed for inflammatory cells, and DNA was extracted from sputum for Chlamydophyla pneumoniae MOMP gene detection using real-time PCR. Results Asthma phenotype was predominantly eosinophilic in children with acute asthma (50\%) but neutrophilic in adults with acute asthma (82\%). The paucigranulocytic inflammatory phenotype was most common in both adults and children with stable asthma. Chlamydophyla pneumoniae was not detected in $99 \%$ of samples.

Conclusions The pattern of inflammatory phenotypes differs between adult and children with asthma, with eosinophilic disease being more prevalent in childhood asthma, and neutrophilic inflammation being the dominant pattern in adults. The aetiology of neutrophilic asthma may not be due to current active Chlamydophyla pneumoniae infection.

Supported by Chinese Scholarship Council.

Conflict of Interest No.

\section{A NOVEL E3 UBIQUITIN LIGASE LINKS RHINOVIRUS INFECTION TO EXACERBATION OF ASTHMA}

L HATCHWELL $^{1}$, A COLLISON $^{1}$, AP SIQUEIRA ${ }^{1}$, PS FOSTER $^{2}$, N VERRILLS ${ }^{3}$, A DON $^{4}$, P WARK $^{5}$, J MATTES ${ }^{1,6}$

${ }^{1}$ Experimental \& Translational Immunology Group, ${ }^{2}$ Centre for Asthma and Respiratory Diseases (CARD), ${ }^{3}$ Medical Biochemistry, School of Biomedical Sciences and Pharmacy, University of Newcastle, ${ }^{4}$ University of NSW, ${ }^{5}$ Respiratory and Sleep Medicine Unit, John Hunter Hospital, and ${ }^{6}$ Paediatric Respiratory and Sleep Medicine Unit, John Hunter Children's Hospital, Newcastle, Australia

Aim To elucidate rhinovirus-induced inflammatory signals that lead to exacerbation of pre-existing allergic airways disease.

Methods Naïve and allergic House Dust Mite (HDM) challenged mice were treated with either siRNA targeted against an E3 ubiquitin ligase or small molecules that reactivate its specific substrate - a protein phosphatase involved in p38 MAPK phosphorylation. Mice were then infected with rhinovirus (RV) 1B and inflammation, Th2 responses, chemokine release, and airways hyperreactivity was assessed.

Results Inhibition of the E3 ubiquitin ligase or activation of its substrate - a protein phosphatase - attenuated rhinovirus-induced exacerbation by suppressing airways hyperreactivity, recruitment of neutrophils and eosinophils to the airway, as well as lowering levels of Th2 cytokines and chemokines such as exotaxin-1. However, expression of type 1 interferons and viral titres were not affected following intervention. We confirmed expression of the E3 ubiqitin ligase in primary airway epithelial cells derived from asthmatics upon ex vivo RV1B infection.

Conclusion This study reveals how inhibition of a novel E3 ubiquitin ligase or reactivation of its protein phosphatase substrate attenuates rhinovirusinduced exacerbation of allergic airways disease independent of affecting antiviral responses or virus replication. Our experimental studies suggest that inhibition of virus-induced inflammation may be of therapeutic value for rhinovirus-induced asthma exacerbations.

Supported by NHMRC

Conflict of Interest No. 


\section{EFFECTS OF BUDESONIDE AND FORMOTEROL ON INNATE} ANTI-VIRAL IMMUNE RESPONSES IN VITRO

JW UPHAM, ML CARROLL, AJ POH, D KIRKEGAARD, H LI, JM DAVIES

The University of Queensland School of Medicine, and Department of Respiratory Medicine, Princess Alexandra Hospital, Brisbane, Australia

Background Rhinoviruses (RV) often trigger acute exacerbations of asthma. While combination treatment with inhaled steroids and long-acting beta agonists is effective at improving asthma control, there is little known about the effects of these medications on the host response to virus infections. Host immunity involves both structural cells of the lungs (e.g. epithelial cells) and leukocytes recruited from the circulation. The aim of this study was to examine the effects of budesonide and formoterol on innate anti-viral immune responses in blood immune cells.

Methods Blood mononuclear cells from asthmatics and healthy donors were activated with either a Toll-like receptor 7 (TLR7) agonist (imiquimod), or with RV16, for up to 24 hours in the presence of budesonide and formoterol $\left(10^{-6}\right.$ to $10^{-10} \mathrm{M}$ ). Cytokines were measured by qPCR and ELISA.

Results Budesonide inhibited RV16- or TLR7-induced type I interferon signalling and IL-6 and IP-10 synthesis in a dose-dependent manner. Formoterol had little effects on these end points, but enhanced the effects of budesonide when used in combination. RV16-induced type I interferon signalling was reduced in asthmatics compared to healthy donors. Both drugs had similar inhibitory effects on mediator synthesis by cells from asthmatic and healthy donors.

Conclusions Budesonide inhibits in vitro synthesis of pro-inflammatory genes and innate interferon induced by RV16 and TLR7 activation. These effects are amplified by simultaneous exposure to formoterol.

Support The Asthma Foundation of QLD, NHMRC.

Conflict of Interest Budesonide and formoterol were supplied by AstraZeneca.

\section{INVESTIGATION OF INFECTION-INDUCED STEROID- RESISTANT ASTHMA}

JC HORVAT ${ }^{1}$, A-T ESSILFIE ${ }^{1}$, RY KIM ${ }^{1}$, JL SIMPSON ${ }^{1}$, ML DUNKLEY $^{1,2}$, KW BEAGLEY ${ }^{3}$, PG GIBSON ${ }^{1}$, PS FOSTER ${ }^{1}$, PM HANSBRO'

${ }^{1}$ Centre for Asthma and Respiratory Diseases and HMRI, University of Newcastle, Newcastle, NSW, ${ }^{2}$ Hunter Immunology LTD, Newcastle, NSW, and ${ }^{3}$ Institute of Health and Biomedical Innovation, Queensland University of Technology, Kelvin Grove, Qld

Neutrophilic asthma (NA) has been associated with increased bacterial colonization of the airways and increased expression of innate immune factors in the lung. This suggests that infection may play an important role in the pathogenesis of NA. NA is an important health issue as sufferers are resistant to steroid treatment, which is the mainstay of asthma therapy and effective therapies are urgently required. Using mouse models of Chlamydia and Haemophilus influenzae lung infection and ovalbumin (Ova)-induced allergic airway disease (AAD), we have shown how infection may be linked to NA. Both infections suppressed eosinophilic inflammation and T-helper (Th) type 2 responses but increase neutrophilic inflammation and innate and Th1 and/or Th17 responses in AAD.

In the current study, the effectiveness of steroid treatment for the suppression of infection-induced neutrophilic AAD was assessed by treating infected Ovasensitized mice intranasally with dexamethasone during Ova challenge. Whilst dexamethasone treatment suppressed Th2-mediated, eosinophilic AAD in uninfected, Ova-sensitized groups, Chlamydia and Haemophilus-induced neutrophilic AAD were shown to be steroid-resistant.

Our findings correlate with clinical observations which show associations between infection, neutrophilic inflammation and steroid resistance in asthmatics. These models will be utilized to examine the effectiveness of a number of novel therapies for infection-induced neutrophilic AAD and to develop improved treatment strategies for steroid-resistant asthma.

Supported by NHMRC, Asthma Foundation of NSW, HMRI.

Conflict of Interest No.

\section{TP-018}

\section{ANALYSIS OF SYSTEMIC GENE EXPRESSION ACCORDING TO} INFLAMMATORY PHENOTYPE OF ASTHMA

KJ BAINES ${ }^{1,2}$, JL SIMPSON $^{1,2}$, RJ SCOTT ${ }^{3}$, LG WOOD ${ }^{1,2}$, PG GIBSON $^{1,2}$ Priority Research Centre's for ${ }^{1}$ Asthma and Respiratory Disease, and ${ }^{3}$ Information Based Medicine, The University of Newcastle, NSW, Australia, and ${ }^{2}$ Respiratory \& Sleep Medicine, HMRI, John Hunter Hospital, NSW, Australia

Rationale Four inflammatory phenotypes of asthma have been identified including eosinophilic, neutrophilic, mixed granulocytic and paucigranulocytic asthma, based on the presence or absence of sputum granulocytes. The involvement of systemic inflammation in the pathogenesis of inflammatory phenotypes of asthma remains unknown.

Objective This study investigates differences in the whole genome gene expression profile of peripheral blood in inflammatory phenotypes of asthma. Methods Induced sputum and peripheral blood were collected from participants with asthma $(n=38)$. Inflammatory cell counts were performed and inflammatory phenotype assigned based on the eosinophil and neutrophil cutoffs of $3 \%$ and $63 \%$, respectively. RNA was extracted from whole blood, gene expression profiles were generated (Illumina Humanref-8 V2) and analysed using Genespring GX11.

Results Participants with eosinophilic asthma had significantly higher rates of atopy and levels of exhaled nitric oxide. There were 7 genes classified as differentially expressed between the 4 asthma phenotypes including the $\alpha$-defensins (DEFA) 1, 1B, 3 and 4 , neutrophil proteases cathepsin G (CTSG) and elastase (ELA2), and the monocyte/macrophage serine esterase, carboxylesterase 1 (CES1). Expressions of DEFA1, 1B, 3, 4, CTSG and ELA2 were significantly higher in neutrophilic asthma and expression of CES1 was significantly higher in mixed granulocytic asthma. Microarray results of the $\alpha$-defensins and neutrophil proteases were successfully validated using realtime PCR.

Conclusions There is systemic up-regulation of $\alpha$-defensins and neutrophil proteases in neutrophilic asthma, and these molecules play an important role in neutrophil activation and migration. Systemic activation of neutrophils is an important feature involved in the pathogenesis of neutrophilic asthma, which is significantly different to other asthma phenotypes.

Supported by HMRI and Xstrata Coal; The University of Newcastle. Conflict of Interest No.

Conflict of interest $\mathrm{No}$ 


\section{DEVELOPMENT OF A SHEEP MODEL TO STUDY THE VARIABLE RESPONSE TO PEANUT ALLERGENS}

\author{
R BISCHOF ${ }^{1}$, J VAN GRAMBERG ${ }^{1}$, M DE VEER ${ }^{1}$, R O'HEHIR', \\ E MEEUSEN $^{1}$ \\ ${ }^{1}$ Biotechnology Research Laboratories, Monash University, Clayton Vic., \\ Australia, and ${ }^{2}$ Department of Allergy, Immunology and Respiratory \\ Medicine, Alfred Hospital, Melbourne Vic., Australia
}

Aim Develop a sheep model for peanut allergy and to begin to examine the innate immune responses in individual sheep following stimulation with peanut allergen.

Methods Sheep were immunized subcutaneously with peanut (Arachis hypogaea) allergen extract and house dust mite extract (HDM). Peripheral blood was collected pre- and post-immunization to determine allergen-specific immunoglobulin (Ig) levels by ELISA. Immediate and delayed-type hypersensitivity (DTH) responses were also assessed following intra-dermal allergen injection. In naïve sheep, pseudo-afferent lymphatic vessels were cannulated to examine lymph cells and fluid draining the skin following injection of peanut allergen extract.

Results A peanut-specific lgE response was detected in 50\% of immunized sheep; those that were allergic to peanut also had strong IgE responses to HDM. High peanut-specific IgE responses also correlated strongly with immediate and delayed skin hypersensitivity responses to peanut allergen extract. Preliminary lymphatic cannulation studies revealed that peanut antigen is taken up by distinct innate immune cell types.

Conclusions The data presented indicates that a proportion of sheep in this model become allergic to peanut allergen, as evidenced by high peanut allergen-specific serum IgE levels and DTH responses, similar to that seen in humans. Sheep responded similarly to both peanut and HDM allergens, implying that the response may not be based solely on the allergen, but rather the way the immune system is responding to the allergen, consistent with genetic predisposition. Distinct innate immune cell types are involved in allergen uptake and may play a key role in determining the allergic phenotype.

Supported by ARC

Conflict of Interest No.

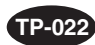

\section{STEREOLOGICAL ESTIMATES OF HYPERPLASIA AND HYPERTROPHY OF AIRWAY SMOOTH MUSCLE - SOURCES OF VARIATION}

R JONES ${ }^{1,2}, \mathrm{~J} \mathrm{ELLIOT}^{1}$, A JAMES A $^{1,2}$

'WA Sleep Disorders Research Institute, Sir Charles Gairdner Hospital, Hospital Avenue, Nedlands, WA, and ${ }^{2}$ School of Medicine and

Pharmacology, University of Western Australia, Crawley, WA

Assessment of airway smooth muscle (ASM) cell size and number requires estimates of cell volume density $\left(\mathrm{N}_{\mathrm{V}}\right)$, volume fraction of muscle $\left(\mathrm{f}_{\mathrm{ASM}}\right)$ within the ASM layer and the volume of ASM per length of airway. Stereological techniques have now become the accepted standard for assessing ASM cell parameters, but sources of variation remain unclear.

Aim To assess sources of variability in the estimation of ASM cell parameters and volume fractions within the ASM layer.

Methods Large and small airways from subjects with and without asthma were examined. Transverse airway sections were cut at $0.5 \mu \mathrm{m}$ and $4 \mu \mathrm{m}$ (Masson's trichrome technique), and $30 \mu \mathrm{m}$ (haematoxylin) and used to estimate ASM cell number and volume, and the volume fraction of muscle $\left(f_{A S M}\right)$ within the layer of ASM. Stereological assessments of the possible sources of variation in these ASM layer parameters were assessed.

Results Increased section thickness overestimated $\mathrm{f}_{\mathrm{ASM}}$ by $<10 \%(0.5 \mu \mathrm{m})$, $20 \%(4 \mu \mathrm{m})$ and $25 \%(30 \mu \mathrm{m})$. Stable variation of $<10 \%$ in $\mathrm{N}_{\mathrm{v}}$ occurred if 40 high-power fields (HPF) were used to estimate $N_{v}$. Variation in the depth of muscle in thick sections of the ASM layer caused up to $10 \%$ overestimation of $N_{v}$. Although the absolute area of the ASM layer varied by up to $30 \%$, variation of $f_{A S M}$ was $<10 \%$ around the airway circumference and along the airway length. $f_{A S M}$ differed significantly between large and small airways.

Conclusion These results suggest that partial thickness HPFs need to be excluded and that $\geq 40 \mathrm{HPF}$ should be used to estimate ASM volume density, that a single $0.5 \mu \mathrm{m}$ section of airway can be used to estimate $\mathrm{f}_{\mathrm{ASM}}$ and that ASM parameters should be compared separately in large and small airways. Grants NHMRC \#446800.

Nominations Nil.

Conflict of Interest Nil.

Journal Compilation (C) 2011 Asian Pacific Society of Respirology

\section{INCREASED AIRWAY SMOOTH MUSCLE CELL SIZE AND NUMBER ARE NOT RELATED TO AGE OR AGE AT ONSET OF ASTHMA}

A JAMES ${ }^{1,2}$, R JONES $^{1}$, T MAUAD $^{3}$, M DOLHNIKOFF $^{3}$, M ABRAMSON $^{4}$,

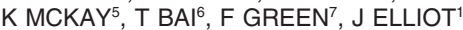

${ }^{1}$ West Australian Sleep Disorders Research Institute, Nedlands, WA, ${ }^{2}$ Medicine and Pharmacology, University of Western Australia, ${ }^{3}$ Sao Paulo University, Sao Paulo, Brazil, ${ }^{4}$ Monash University, Melbourne, Australia, ${ }^{5}$ Children's Hospital at Westmead, Sydney, Australia, ${ }^{6}$ University of British Columbia, Vancouver, BC, Canada, and 'University of Calgary, Calgary, Alta, Canada

The thickness of the airway smooth muscle (ASM) layer is related to severity but not duration of asthma or age (James 2009 ERJ;34:1040). It is unknown if the constituents of the ASM layer change with age.

Aim To investigate the relation of mean ASM cell volume $\left(V_{c}\right)$, total number of cells per mm of airway $\left(\mathrm{N}_{\llcorner}\right)$and fractions of ASM (f $\left.\mathrm{f}_{A S M}\right)$ and extracellular matrix $\left(\mathrm{f}_{\mathrm{ECM}}\right)$ within the ASM layer with age and age at onset of asthma.

Methods Post-mortem tissues from control subjects $(C n=51)$; non-fatal (NFA $n=49)$ and fatal $(F A n=55)$ cases of asthma were used. The volume density $\left(\mathrm{N}_{\mathrm{v}}\right)$ of ASM cell nuclei was estimated on $30 \mu \mathrm{m}$ transverse airway sections (haematoxylin) and mean cell volume $\left(V_{C}=1 / N_{V}\right)$ was calculated, correcting for the volume fraction of ASM within the ASM layer. $\mathrm{f}_{\mathrm{ASM}}$ and $\mathrm{f}_{\mathrm{ECM}}$ were estimated on 0.5- $\mu \mathrm{m}$ thick sections of the same airway (Masson's trichrome). Effects of age on ASM cell parameters and tissue volume fractions were tested using general linear models, correcting for sex and study centre and by comparing age at onset of asthma ( $<16$ vs. $>16$ years).

Results Table shows age at onset for asthma groups in large airways (case means \pm SD)

\begin{tabular}{lcccc}
\hline & $\mathrm{V}_{\mathrm{C}}, \mu \mathrm{m}^{3}\left(\mathrm{x} 10^{4}\right)$ & $\mathrm{N}_{\mathrm{L}}, \mathrm{mm}^{3}\left(\mathrm{x} 10^{4}\right)$ & $\mathrm{f}_{A S M}$ & $\mathrm{f}_{\mathrm{ECM}}$ \\
\hline NFA $<16$ & $3.5 \pm 0.8$ & $17.2 \pm 10.1$ & $0.65 \pm 0.04$ & $0.19 \pm 0.05$ \\
NFA $>16$ & $3.6 \pm 1.2$ & $17.0 \pm 10.4$ & $0.64 \pm 0.07$ & $0.20 \pm 0.05$ \\
FA $<16$ & $3.9 \pm 1.2$ & $27.4 \pm 12.7$ & $0.67 \pm 0.08$ & $0.16 \pm 0.05$ \\
FA $>16$ & $3.9 \pm 1.6$ & $19.3 \pm 11.0$ & $0.67 \pm 0.13$ & $0.14 \pm 0.05$ \\
\hline
\end{tabular}

No significant correlation was seen with age for any ASM cell parameters or tissue fractions. Results were similar for medium and small airways.

Conclusion Size and number of ASM cells and the volume fractions of ASM and ECM within the layer of ASM are not related to age.

Support NHMRC Australia (Grants \#343601; \#446800).

Nomination Nil.

Conflict of Interest No. 
DEEP INSPIRATION PRIOR TO BRONCHOCONSTRICTOR CHALLENGE HAS BENEFICIAL AND DETRIMENTAL EFFECTS ON LUNG MECHANICS IN MICE

\author{
RR WONG ${ }^{1,2}$, AN LARCOMBE $^{1}$, LB FERNANDES ${ }^{2}$, GR ZOSKY $^{1}$, \\ PB NOBLE ${ }^{1,3}$ \\ ${ }^{1}$ Telethon Institute for Child Health Research, Centre for Child Health \\ Research, ${ }^{2}$ Pharmacology and Anaesthesiology Unit, School of Medicine \\ and Pharmacology, and ${ }^{3}$ School of Biomedical, Biomolecular and Chemical \\ Sciences, University of Western Australia, WA, Australia
}

Background In healthy humans, deep inspiration (DI) prior to bronchoconstrictor challenge attenuates the fall in $\mathrm{FEV}_{1}$, a phenomenon dubbed 'bronchoprotection'.

Aim To assess whether the effects of DI prior to bronchoconstrictor challenge are due to: (1) reduced airway narrowing; (2) reduced airway closure; and/or (3) enhanced bronchodilation to DI preceding maximal expiration.

Methods Anaesthetized and mechanically ventilated mice (BALB/c) received two single-dose methacholine $(\mathrm{MCh})$ challenges $(30 \mathrm{mg} / \mathrm{mL})$ that were either preceded by 5 DIs, or by 20 minutes of ventilation without DI. In a separate group of mice, DI was also induced at the peak of constriction after challenge to assess changes in the bronchodilatory response to DI. DI was simulated by mechanical inflation to a transrespiratory pressure of $30 \mathrm{cmH}_{2} \mathrm{O}$. Airway narrowing and bronchodilation to $\mathrm{DI}$ were assessed from changes in airway resistance, and airway closure from the change in tissue elastance, measured by the forced oscillation technique.

Results MCh challenge produced an increase in airway resistance (i.e. airway narrowing) which was enhanced when challenge was preceded by DI $(p<0.001, n=8)$. In contrast, MCh had no effect on tissue elastance after either challenge, suggesting that airway closure did not occur irrespective of the challenge protocol. DI induced after challenge produced immediate bronchodilation which was greater when challenge was preceded by $\mathrm{DI}(\mathrm{p}<0.05$, $\mathrm{n}=8$ ).

Conclusions DI prior to bronchoconstrictor challenge has both beneficial and detrimental effects on lung function in mice. Findings support the possibility that the beneficial effects of prior DI in healthy humans are related to enhanced bronchodilation to DI preceding maximal expiration.

Supported by NHMRC \& University of Western Australia.

Nomination No.

Conflict of Interest No.

TP-025

\section{ISOLATED HUMAN BRONCHI DILATE TO DEEP INSPIRATION} IN AN AMPLITUDE-DEPENDENT MANNER

PK MCFAWN ${ }^{1}, \mathrm{RL}^{\mathrm{J}} \mathrm{JONES}^{2}$, A CAIRNCROSS $^{1}$, HW MITCHELL ${ }^{1}$, AL JAMES ${ }^{2}$, PB NOBLE ${ }^{1,3}$

${ }^{1}$ School of Biomedical, Biomolecular and Chemical Sciences, University of Western Australia, ${ }^{2}$ Department of Pulmonary Physiology, Sir Charles Gairdner Hospital, WA, Australia, ${ }^{3}$ Telethon Institute for Child Health

Research, Centre for Child Health Research, University of Western Australia

Background Deep inspiration (DI) produces a bronchodilator response in healthy humans, but this response is impaired in asthma. Reduced airway compliance in disease could impair the response to DI by limiting the stretch of smooth muscle.

Aim To show that isolated human bronchi dilate to DI in an amplitudedependent manner and that the stretch caused by DI depends on airway compliance.

Methods Bronchi were obtained following lung resection from cancer patients who had normal spirometry $(n=8)$. Lumen narrowing was measured using a servo-control system which set transmural pressure and simulated breathing movements. Bronchi were contracted to carbachol (CCh $\left.3 \times 10^{-6} \mathrm{M}\right)$ during tidal breathing (from 5 to $10 \mathrm{cmH}_{2} \mathrm{O}$, i.e. $\Delta 5 \mathrm{cmH}_{2} \mathrm{O}$ transmural pressure, $0.25 \mathrm{~Hz})$ and inflated to three different amplitudes of $\mathrm{DI}\left(\Delta 10,25\right.$ or $\left.55 \mathrm{cmH}_{2} \mathrm{O}\right)$ applied following contraction.

Results In CCh-contracted airways, all three DI amplitudes produced a transient bronchodilation. Increasing the DI amplitude caused a greater increase in luminal volume during the DI and a greater bronchodilation following the DI $(p<0.05)$. CCh itself cause approximately a $20 \%$ fall in specific compliance $(p<0.05)$, which was reversed by DI $(p<0.05)$. For each DI amplitude, the change in lumen volume during the DI was positively correlated to the specific compliance of the bronchi before DI $(r>0.87, p<0.01)$

Conclusions Isolated human bronchi show a bronchodilation response to DI that is proportional to the expansion of the airway caused by the DI. The amount of stretch produced by a DI depends on airway wall compliance suggesting that increased airway stiffness in disease could suppress the DI response by limiting the stretch of bronchi during lung inflation.

Conflict of Interest None.
DIRECT MEASUREMENT OF AIRWAY NARROWING IN CULTURED WHOLE AIRWAY SEGMENTS - A NEW METHOD TO ASSESS FUNCTIONAL OUTCOMES IN CULTURE

TK ANSELL ${ }^{1}$, PB NOBLE ${ }^{1,2}, \mathrm{HW}$ MITCHELL ${ }^{1}, \mathrm{PK} \mathrm{MCFAWN}^{1}$

${ }^{1}$ School of Biomedical, Biomolecular and Chemical Sciences, University of Western Australia, and ${ }^{2}$ Telethon Institute for Child Health Research, Centre for Child Health Research, University of Western Australia, WA, Australia

Background Asthma is characterized by excessive airway narrowing to contractile stimuli, termed airway hyper-responsiveness (AHR). Changes in airway smooth muscle (ASM) protein expression or mass are possible contributing mechanisms underlying AHR and have been examined using cell culture techniques. However, how these cellular changes to ASM relate to airway narrowing at the level of the whole airway is unclear. We describe a new method to track changes in airway narrowing (responsiveness) in culture.

Methods Whole airway segments (generation 12-17) from sheep lungs were studied prior to (fresh) and after 24 and 48 hours in culture in Dulbecco's Modified Eagle Medium with 2\% bovine serum albumin, 1\% L-glutamine and antibiotics. Airway narrowing was measured from the \% decrease in airway volume under a fixed transmural pressure, using a servo-controlled syringe pump and organ bath apparatus. Cumulative acetylcholine dose-response curves (ACh, $10^{-7} \mathrm{M}-3 \times 10^{-3} \mathrm{M}$ ) were performed to determine maximal response $\left(E_{\max }\right)$ and sensitivity $\left(\mathrm{pD}_{2}\right.$, negative log of $\left.E C_{50}\right)$.

Results Fresh airway segments narrowed strongly and approached closure with an $E_{\max }$ of $89.7 \% \pm 9.5( \pm S E M)$ and $\mathrm{pD}_{2}$ of $4.4 \pm 0.1$. Airway narrowing responses were preserved in culture, with no significant difference in maximal response or sensitivity to $A C h$ after either $24\left(E_{\max } 89.6 \% \pm 4.7, p_{2} 4.8 \pm 0.3\right)$ or 48 hours in culture $\left(E_{\max } 84.4 \% \pm 5.1, \mathrm{pD}_{2} 4.4 \pm 0.3\right)$.

Conclusions The present study has validated a new method allowing changes occurring at the cellular level in culture to be related to changes in airway responsiveness at the whole airway level. Future studies will assess the effects of chronic inflammation in disease on airway responsiveness.

Supported by NHMRC.

Nomination Janet Elder International Travel Awards.

Conflict of Interest No.

\section{ASTHMA \& ALLERGY SIG: POSTER SESSION 3}

THE PREVALENCE OF ASTHMA AND COPD IN A REPRESENTATIVE SAMPLE OF OLDER AUSTRALIANS

JA DOUGLASS ${ }^{1,2,3}$, EA YU $U^{1,2}$, BR THOMPSON $N^{1,2,3}$, GG KING $^{2,4}$, MJ ABRAMSON ${ }^{2,5}$

${ }^{1}$ Department of AIRmed, Alfred Hospital, Vic., Australia, ${ }^{2}$ Cooperative Research Centre for Asthma and Airways, NSW, Australia, ${ }^{3}$ Department of Medicine, Monash University, Vic., Australia, ${ }^{4}$ Woolcock Institute of Medical Research, NSW, Australia, and ${ }^{5}$ Department of Epidemiology and Preventive Medicine, Monash University, The Alfred, Vic., Australia

Aim To estimate the prevalence of asthma and COPD among adults aged 65 years and over.

Methods A representative sample of 5002 randomly selected older people was posted a questionnaire on respiratory symptoms. Non-responders received 2 additional mailouts and were contacted by telephone. Data were entered into SPSS and descriptive statistics are reported as proportions (95\% confidence intervals).

Results Questionnaires were completed by 3161 (70\%) participants, of whom $1760(56 \%)$ were female and the mean $( \pm S D)$ age was $77( \pm 7.4)$. Wheezing at any time in the past 12 months was reported by $18.2 \%(95 \% \mathrm{Cl}$ $16.8-19.5 \%)$. Asthma was reported by $15(13.8-16.3 \%)$ of participants and of these, $88 \%$ confirmed a doctor-diagnosis of asthma $(13,12-14.4 \%$ of all participants). Doctor-diagnosed COPD was reported by $6.5(5.6-7.3 \%)$, and chronic bronchitis by $7.8(6.9-8.8 \%)$. Almost half $(45.2,43.4-46.9 \%)$ had smoked regularly. Phlegm and sputum production in the winter was reported by $15.7(14.4-17 \%)$ of participants.

Conclusions Our findings show almost 1 in 5 people aged 65 years and over report wheeze which may indicate asthma. Surprisingly, in this representative sample, the prevalence of doctor-diagnosed asthma was high at $15 \%$. Doctor-diagnosed COPD was present in $6.5 \%$ of participants, but higher levels of symptomatic bronchitis and sputum production suggests substantial underdiagnosis of COPD in this group.

Supported by The Cooperative Research Centre for Asthma and Airways. Conflict of Interest No.

Journal Compilation @ 2011 Asian Pacific Society of Respirology 


\section{DEVELOPMENT AND VALIDATION OF THE PATIENT ASTHMA CONCERNS TOOL (PACT) TO IDENTIFY THE NEEDS OF OLDER PEOPLE WITH ASTHMA}

\author{
MA CRANE ${ }^{1,2}$, JA DOUGLASS ${ }^{1,3}$, DP GOEMAN ${ }^{1,3}$, NE COUSENS ${ }^{1,2}$, \\ CR JENKINS ${ }^{1,2}$ \\ ${ }^{1}$ CRC for Asthma and Airways, ${ }^{2}$ Clinical Management, Woolcock Institute \\ of Medical Research, Sydney, NSW, Australia, and ${ }^{3}$ Allergy, Immunology \\ \& Respiratory Medicine, The Alfred Hospital\& Monash University, Vic., \\ Australia
}

Aim Tools which assist practitioners to identify patient concerns and tailor consultations to meet their needs can improve the delivery of care and adherence to treatment. This study aimed to test the validity and reliability of a tool designed to identify the particular unmet needs and concerns of older people with asthma.

Methods The Patient Asthma Concerns Tool (PACT) is a 14-item self-completed questionnaire, developed from a pool of items used to investigate the needs of older people with asthma. After pilot testing, the initial survey was administered to a small convenience sample $(n=30)$ and then a communitybased sample $(n=193)$, psychometrically tested, refined and then evaluated by clinicians $(n=30)$.

Results The PACT demonstrated good content and construct validity and was highly rated by the clinicians. It correlated well with the ACQ and adherence scores on related variables and was able to discriminate between poor and well-controlled asthma and on medication adherence. High ACQ scores were well correlated with reporting of moderate-extremely severe symptoms $(p<0.0001)$, poor control $(p=0.002)$, any restriction $(p=0.0003)$, frustration $(p=0.006)$ or emergency GP visit $(p=0.001)$. Reporting of preventer adherence also correlated with adherence scores $(p=0.0005)$. The PACT also showed good reliability with good-excellent Kappa/ICC scores. Internal consistency of factors was high, and the overall Cronbach's alpha was 0.649 . Conclusion The PACT questionnaire was shown to be a valid and reliable tool clinically and psychometrically. Its use in primary care settings should assist health professionals to identify the particular needs and concerns of older patients with asthma and direct tailored asthma management accordingly.

Supported by Co-operative Research Centre for Asthma and Airways. Conflict of Interest No.

\section{A RANDOMIZED CONTROLLED TRIAL OF NEEDS-BASED ASTHMA EDUCATION IN OLDER PEOPLE WITH ASTHMA}

JA DOUGLASS ${ }^{1,2}$, D GOEMAN ${ }^{1,2}$, M CRANE $^{1,3}$, C JENKINS $^{1,3}$

${ }^{1}$ Co-operative Research Centre for Asthma and Airways, Camperdown, NSW, ${ }^{2}$ AlRMed, The Alfred Hospital \& Monash University, Melbourne,

Vic., and ${ }^{3}$ The Woolcock Institute of Medical Research, Glebe, NSW

Aim To develop and evaluate an educational intervention utilizing the self completed Patient Asthma Concerns Tool (PACT) for older people with asthma.

Methods A randomized, placebo-controlled, single-blinded trial of tailored asthma education including device technique and utilizing PACT to address patients' concerns versus brochure-only information for asthma patients over age 55. Measurements of lung function, asthma control (ACQ), asthma related quality of life (AQoL), medication use and adherence score $(A D H)$ were obtained at baseline, 3 and 12 months using standard, validated questionnaires.

Results Sixty-five participants (49F $16 \mathrm{M}$, mean age $68 \pm 8.3$ ) were randomized to the intervention group and $58(40 \mathrm{~F} 18 \mathrm{M}$, mean age $67 \pm 6.4)$ to the control. There were no statistically significant differences between the groups' demographics or baseline measurements. A Wilcoxon Signed Ranks Test used to compare median pair ranking at baseline and 3 months post-intervention revealed a significant improvement in the active, but not the brochure-only information group at 3 months in:

$\mathrm{ACQ}$ mean $\pm \mathrm{SD}=1.5 \pm 1.02$ vs. $1.2 \pm 0.8(\mathrm{p}=0.005)$

AQoL mean $\pm S D=1.1 \pm 0.8$ vs. $0.8 \pm 0.6(p=0.003)$.

$\mathrm{ADH}$ mean $\pm \mathrm{SD}=1.4 \pm 1.3$ vs. $0.6 \pm 0.8(\mathrm{p}<0.001)$.

Conclusion An educational intervention including device technique and addressing the concerns of older people with asthma significantly improved $A C Q, A Q O L$ and ADH scores at 3 months post-intervention.

Australian New Zealand Clinical Trials Registry ACTRN 12609000639224 Supported by The Co-operative Research Centre for Asthma and Airways. Nomination None.

Conflict of Interest No.

Journal Compilation (C) 2011 Asian Pacific Society of Respirology

\section{DIET QUALITY IS POOR IN SEVERE ASTHMATICS COMPARED TO HEALTHY CONTROLS}

BS BERTHON ${ }^{1,2}$, L MACDONALD-WICKS $^{3}$, PG GIBSON ${ }^{1,2}$, LG WOOD ${ }^{1,2}$ ${ }^{1}$ School of Biomedical Sciences and Pharmacy, University of Newcastle, NSW, ${ }^{2}$ Respiratory and Sleep Medicine, Hunter Medical Research Institute, John Hunter Hospital, Newcastle, NSW, and ${ }^{3}$ School of Health Sciences, University of Newcastle, NSW

Introduction Increasing asthma prevalence and changes in environmental exposure suggest that there may be a relationship between asthma and dietary intake. However, to date, few studies have examined how dietary intakes of asthmatics differ from a healthy population.

Aim To measure and compare the dietary intakes of adults with stable asthma and healthy controls.

Methods In a cross-sectional study, dietary intakes calculated from a 186item food frequency questionnaire (FFQ) of adults with stable asthma $(n=110$, age 56 years \pm 15 (SD)) were compared with intakes of healthy controls $(n=$ 65 , age 47 years \pm 17 (SD)) matched for age and body mass index (BMI). Spirometry, airway responsiveness to hypertonic saline, and induced sputum cell counts were also measured.

Results Subjects with severe persistent asthma $(n=59)$ had significantly higher total fat intake than healthy controls (103 \pm 49 (SEM) versus $98 \pm 4$ (SEM) $g /$ day $p=0.014$ ) and significantly lower fibre intakes (32 \pm 11 (SEM) versus $37 \pm 13(\mathrm{SEM}) \mathrm{g} /$ day $p=0.018)$. Lower fibre intake in asthmatic subjects $(n=124)$ was associated with lower \%predicted FEV $1(r=0.41, p=0.004)$, $\% F V C(r=0.43, p=0.015)$ and $F_{E V} / F V C(r=0.44, p=0.048)$. Higher fat intake and lower fibre intake were associated with higher absolute concentrations of sputum eosinophils $(r=0.42, p=<0.001, n=107)$.

Conclusions Subjects with severe persistent asthma have an eating pattern of lower diet quality with higher intakes of fat and lower intakes of fibre than healthy controls, which is related to lower lung function and increased airway inflammation. Factors leading to altered dietary intake in severe asthma require further investigation.

Conflict of Interest No.

\section{SUCCESS IN A WEIGHT LOSS TRIAL IS RELATED TO ASTHMA SEVERITY}

HA SCOTT ${ }^{1,2}$, PG GIBSON ${ }^{1,2}$, ML GARG $^{3}$, JJ PRETTO ${ }^{1,2}$, PJ MORGAN $^{4}$, R CALLISTER ${ }^{3}$, LG WOOD ${ }^{2,3}$

${ }^{1}$ School of Medicine and Public Health, University of Newcastle, NSW, ${ }^{2}$ Department of Respiratory and Sleep Medicine, HMRI, John Hunter Hospital, NSW, ${ }^{3}$ School of Biomedical Sciences and Pharmacy, University of Newcastle, NSW, and ${ }^{4}$ School Education, University of Newcastle, NSW

Introduction Weight loss is recommended for overweight and obese adults with asthma, but can be difficult to achieve for many individuals. The aim of this study was to determine if success in a weight loss program was related to baseline asthma characteristics or food behaviours.

Methods Overweight and obese (BMI $\left.28-40 \mathrm{~kg} / \mathrm{m}^{2}\right)$ adults with asthma were randomized to either a calorie-restricted diet $(n=11)$, physical activity intervention $(n=9)$, or combined caloric restriction and physical activity intervention $(n=10)$ for 10 weeks. Subjects underwent pre- and post-intervention spirometry and weight measurement. Quality of life was determined using the Asthma Quality of Life Questionnaire (AQLQ), and eating behaviours were assessed using the Three-Factor Eating Questionnaire (TFEQ-R18).

Results Median (IQR) weight loss was $6.7(2.3,10.1) \%$ of body weight. Those who lost more weight had a lower baseline FEV 1 /FVC $(r=-0.381, p=$ $0.038)$ and poorer asthma-related quality of life $(r=-0.410, p=0.024)$, including more severe activity limitation $(r=-0.442, p=0.015)$ and asthma symptoms $(r=-0.384, p=0.036)$. There was no relationship between weight loss and baseline uncontrolled eating $(p=0.952)$, emotional eating $(p=0.280)$ or cognitive restraint $(p=0.497)$, measured by the TFEQ-R18.

Conclusions Severity of asthma, when expressed as degree of airflow obstruction and AQLQ, was predictive of weight loss success, but surprisingly, baseline eating behaviour was not. This indicates that asthma severity may be an important motivator for weight loss in adults with asthma.

Supported by CCRE NHMRC Postgraduate Scholarship; HMRI Postgraduate Student Support Package, sponsored by the Greaves family.

Conflict of Interest No. 
TIME SPENT OUTDOORS IS INVERSELY ASSOCIATED WITH REPORTED DIAGNOSES OF ASTHMA AND HAYFEVER IN OLDER AUSTRALIANS

H VALLY ${ }^{1}$, F VERSTER $^{2}$, R LUCAS ${ }^{1}$, NL MISSO ${ }^{3}$, AM HUGHES $^{1}$

${ }^{1}$ The National Centre for Epidemiology and Population Health, The Australian National University, ACT, Australia, ${ }^{2}$ Faculty of Medicine and Heath Sciences, The University of Auckland, New Zealand, and ${ }^{3}$ Lung Institute of Western Australia, The University of Western Australia, Perth, Australia

Introduction Greater exposure to ultraviolet radiation (UV) may increase the risk of allergic disease, but this association has not been investigated using estimates of time spent outdoors by individuals. The aim of this study was to investigate the relationship between self-reported doctor-diagnosed asthma and/or hayfever, and time spent outdoors.

Methods This analysis was based on cross-sectional baseline data from a subsample of the Australian 45 and Up Study, comprising 15678 men and 20155 women aged 45-74 years, living in New South Wales. Participants were randomly selected from the Australian universal health insurance database. Diagnoses of asthma and/or hayfever and the number of hours spent outdoors were derived by questionnaire.

Results In general, the odds of a diagnosis of asthma and/or hayfever decreased with increasing time spent outdoors for both women and men. For example, in women, the adjusted odds ratios for asthma with hayfever were 0.76 (95\% Cl: $0.52-1.13), 0.69(0.47-0.99), 0.66(0.45-0.97)$ and $0.58(0.40-$ 0.83 ) for $1-2,2-3,3-4$ and $>4$ hours spent outdoors on weekends, respectively, compared with $<1$ hour ( $p_{\text {trend }}<0.0001$ ). Time spent outdoors was not associated with a diagnosis of asthma alone in men.

Conclusions There were statistically significant inverse associations between time spent outdoors and diagnoses of asthma, hayfever or asthma with hayfever, in a large population of older Australians. Exposure to UV may protect against the development of allergic diseases, such as asthma and hayfever.

Conflict of Interest No.

\section{ASSOCIATION BETWEEN REPRODUCTIVE FACTORS AND ALLERGIC RHINITIS AND ECZEMA}

MYZ LAU ${ }^{1}$, P SAMARASINGHE ${ }^{1}$, JA BURGESS ${ }^{1}, K^{\prime}$ ADAMS $^{1}$, MJ ABRAMSON $^{2}$, HE WALTERS ${ }^{3}$, SC DHARMAGE ${ }^{1}$, MC MATHESON ${ }^{1}$ ${ }^{1}$ MEGA Epidemiology, University of Melbourne, Vic., ${ }^{2}$ Department of Epidemiology \& Preventive Medicine, Monash University, Melbourne, Vic., and ${ }^{3}$ Menzies Research Institute, University of Tasmania, Hobart, Australia

Background Allergic rhinitis (AR) and eczema are highly prevalent and females are more commonly affected than males in adulthood. Although there have been extensive studies on AR and eczema in females, little is known about the effect of reproductive factors and the development of late-onset AR/ eczema. We examined potential associations between reproductive factors and AR and eczema using the Tasmanian Longitudinal Health Study (TAHS) data.

Methods The TAHS is a population-based cohort study of respiratory disease. Two thousand seven hundred sixty-four $(32.2 \%)$ females from the original 8583 TAHS participants were surveyed in 2004 using postal questionnaire which collected information on reproductive factors such as ever pregnancy, age at first child birth, use of oral contraceptive pills (OCP) and age of starting using the OCP. Logistic regression was used to assess the predictors of AR and eczema and all analyses were mutually adjusted.

Results Of the 2764 participants, $45.4 \%(n=1256)$ had late-onset AR and $35.4 \%(n=978)$ had late-onset eczema. Maternal and paternal atopy were significantly associated with AR $(p<0.0001)$. The risk of developing eczema was decreased significantly with increasing age at first menstruation (OR: 0.94, $95 \% \mathrm{Cl}: 0.89-1.00)$ and the increased age at birth of first child $(0.99,0.97-$ 1.00). A decreased risk in AR was observed with the increasing number of pregnancies $(0.89,0.81-0.98)$. However, the associations between age of starting using OCP and AR/eczema were not significant.

Conclusion Later age at start of menses and later age at first pregnancy were associated with a reduced risk of eczema which may be related to hormonal dysregulation.

Grant Support NHMRC Clifford Craig Foundation of Tasmania and Victorian \& Tasmanian Asthma Foundations.

Conflict of Interest No.

\section{AIRWAY RESPONSIVENESS AT 6 AND 11 YEARS IS ASSOCIATED WITH ASTHMA AT 18 YEARS}

DW COX ${ }^{1,2}$, D MULLANE", ${ }^{1,2}$ SW TURNER ${ }^{1,2}$, G ZHANG ${ }^{2}$, CM HAYDEN $^{2}$, SM STICK ${ }^{1}$, J GOLDBLATT ${ }^{2}$, LI LANDAU ${ }^{2}$, PN LE SOUEF ${ }^{1,2}$

${ }^{1}$ Department of Paediatric Respiratory Medicine, and ${ }^{2}$ School of Paediatrics and Child Health, Princess Margaret Hospital, Perth, Australia

Introduction Asthma is the most common chronic childhood disease in Australia. Increased airway responsiveness (AR) is associated with asthma but not all individuals with increased AR have asthma. The Perth infant asthma follow-up study recruited a birth cohort of individuals who have undergone longitudinal assessments of many factors associated with childhood AR. Our previous work reported an association between increased AR in infancy and asthma at 6 and 11 years.

Aim To look at the relationship of increased AR and asthma in early adulthood at different time points from birth.

Methods Individuals were recruited from among expectant parents attending an antenatal clinic at a local metropolitan clinic. At ages 1, 6 and 12 months and again at 6,11 and 18 years, participants underwent an assessment that included a respiratory questionnaire and determination of AR (as evidenced by dose-response slope (DRS) to histamine using the rapid technique).

Results 253 children were initially recruited and studied in infancy. Two hundred three, 174, 147, 103, 176 and 137 children subsequently had AR assessed at 1, 6 and 12 months, 6,11 and 18 years, respectively. There was a significant relationship between DRS at 6 and 11 years and for both asthma at 18 years $(p=0.001$ and $p<0.001$, respectively) and 'wheeze in the past year' at 18 years $(p=0.03$ and $p=0.029$, respectively). There was no significant relationship between DRS in infancy and asthma at 18 .

Conclusion AR at 6 and 11 years is associated with asthma at 18 years. In this study, there was no significant relationship between AR in infancy and asthma at 18 years.

Supported by NHMRC grant.

Conflict of Interest None.
TP-035

\section{THE ROLE OF POLLEN DURING INFANCY ON CHILDHOOD ASTHMA AND ALLERGIC RHINITIS}

B ERBAS ${ }^{1}$, A LOWE$^{2}$, C LODGE ${ }^{2}$, M MATHESON ${ }^{2}$, D VICENDESE ${ }^{1}$, C HOSKING ${ }^{3}$, D HILL $^{3}$, K ALLEN $^{3}, M^{2}$ ABRAMSON ${ }^{4}$, S DHARMAGE $^{2}$

${ }^{1}$ School of Public Health La Trobe University, Melbourne, Vic., Australia,

${ }^{2}$ School of Population Health, The University of Melbourne, Melbourne, Vic., Australia, ${ }^{3}$ Murdoch Children's Research Institute, Royal Children's Hospital, Melbourne Vic., Australia, and ${ }^{4}$ Department of Epidemiology and Preventive Medicine, Monash University, Melbourne, Vic., Australia

Background Although some studies have found that birth during the pollen season increases the risk of asthma later in life, none have used quantitative measures of pollen load.

Aim To examine the association between measures of pollen exposure in early life, and risk of childhood asthma, allergic rhinitis and eczema.

Method Prior to birth, 620 children with a family history of allergic disease were recruited into the Melbourne Atopy Cohort study (MACS). Ambient pollen concentrations were collected for the 495 children born between October 1991 and June 1994. Logistic regression models were used to examine associations between pollen concentrations, eczema at 2 years and allergic diseases at age $6 / 7$ years.

Results Childhood hay fever was associated with grass pollen observed at birth $(p=0.01), 1$ day after birth $(p=0.04)$ and in the first 6 months of life (OR $=1.1195 \% \mathrm{Cl} 1.02-1.21, p=0.02)$. Children at age $6 / 7$ years had a greater risk of asthma if exposed to higher concentrations of non-grass pollen (predominately weeds and herbs) at birth $(p=0.02)$ as well as in the first 6 months of life $(\mathrm{OR}=1.0395 \% \mathrm{Cl} 1.01,1.06, \mathrm{p}=0.006)$. Eczema at 2 years was not associated with exposure to pollen at birth or in the first 6 months of life.

Conclusion These findings suggest that exposure to high levels of pollen in early life may increase children's risk of asthma and allergic rhinitis at the age of 6-7 years.

Grant Support NHMRC.

Conflict of Interest None.

Journal Compilation (C) 2011 Asian Pacific Society of Respirology 
HUMAN RHINOVIRUS (HRV) C IS AS COMMON IN CHILDREN WITH HRV WHO REQUIRED EMERGENCY TREATMENT FOR AN ACUTE RESPIRATORY ILLNESS AS SYMPTOMATIC SIBLING CONTROLS

PG DAVIS ${ }^{1}$, DW COX ${ }^{1}$, S-K KHOO ${ }^{1}$, J BIZZINTINO ${ }^{1}$, EN SCHULTZ1, W-M LEE ${ }^{2}$, GC GEELHOED ${ }^{1}$, JE GERN ${ }^{2}$, J GOLDBLATT ${ }^{1}$, PN LE SOUEF ${ }^{1}$, IA LAING 1,3

${ }^{1}$ School of Paediatrics \& Child Health, University of Western Australia, ${ }^{2}$ Department of Paediatrics, University of Wisconsin-Madison, Madison, WI, USA, and ${ }^{3}$ Telethon Institute for Child Health Research

The PCAAS has found that $87.5 \%$ of children with acute asthma presenting to the Princess Margaret Hospital for Children Emergency Department (PMH ED) had HRV, of which $69 \%$ were HRV group C. Furthermore, HRVC was associated with more severe attacks. However, the prevalence of HRVC in the community is unknown.

Aim To test the hypothesis that HRVC would be found more often in children requiring emergency treatment for an ARI than sibling controls and determine the impact of days since symptoms began on the prevalence of HRV detection in children with an acute respiratory illness (ARI) and sibling controls (Sibs).

Methods ARI $(n=38)$ had nasal samples collected on presentation to the PMH ED and Sibs with symptoms of a cold $(n=25)$, within 1 week of ARI recruitment. Viral RNA was extracted and reverse transcribed. A two-step PCR of the HRV 5' UTR was used for detection, followed by DNA sequencing for typing.

Results ARI and Sibs were $71 \%$ and $48 \%$ male, $32 \%$ and $20 \%$ asthmatic with mean ages of 3.4 and 4.0 years, respectively. HRV +ve ARI $(n=21$, mean $\pm \mathrm{SD}$ days of symptoms $=4.6 \pm 6.1), \mathrm{HRV}-\mathrm{ve} \mathrm{ARI}(\mathrm{n}=14,8.2 \pm 15.2), \mathrm{HRV}$ +ve Sibs $(n=10,2.8 \pm 2.1)$ and HRV -ve Sibs $(n=15,7.1 \pm 4.7)$. Of the 22 and $10 \mathrm{HRV}+\mathrm{ve}$ ARI and Sibs, $76 \%$ and $70 \%$ had HRVC.

Conclusions HRVC is as common in children who have HRV but do/do not require hospital treatment. Detection of HRV is more likely when the nasal sample is collected soon after the appearance of cold symptoms.

Support NHMRC program grant.

Nomination Nil.

Conflict of Interest Nil.

\section{ABNORMAL VOCAL CORD FUNCTION IN DIFFICULT-TO-TREAT ASTHMA}

K LOW 1 , KK LAU ${ }^{2}$, P HOLMES ${ }^{1}$, M CROSSETT $^{2}$, N VALLANCE ${ }^{3}$, D PHYLAND ${ }^{4}$, K HAMZA $^{5}$, G HAMILTON ${ }^{1,6}$, PG BARDIN ${ }^{1,6}$

${ }^{1}$ Respiratory \& Sleep Medicine, ${ }^{2}$ Diagnostic Imaging, ${ }^{3}$ Surgery, ${ }^{4}$ Speech

Therapy and ${ }^{5}$ Statistics, Monash University and Medical Centre, and

${ }^{6}$ Monash Institute of Medical Research, Melbourne, Australia

Introduction Upper airway dysfunction may make asthma more difficult to control and should be suspected in asthmatics refractory to prescribed medical therapy.

Aim A novel imaging technique, dynamic 320-slice computerized tomography (CT), was used to examine laryngeal behaviour in healthy and asthmatic individuals.

Method Vocal cord movement was imaged using 320-slice CT larynx. Healthy volunteers were studied to develop and validate an analysis algorithm for quantification of normal vocal cord function. Further studies were then conducted in 46 patients with difficult-to-treat asthma. In eight severe asthmatics with abnormal vocal cord movement, asthma outcomes were measured after speech therapy.

Results Vocal cord movement was quantified over the breathing cycle by CT using the ratio of vocal cord diameter to tracheal diameter. Normal limits were calculated, validated and applied to evaluate difficult-to-treat asthma. Vocal cord movement was abnormal with excessive narrowing in 23 of 46 $(50 \%)$ asthmatics and severe in nine $(19 \%)$ patients (abnormal $>50 \%$ of inspiration or expiration time). After speech therapy in a small subgroup, asthma symptoms and morbidity improved.

Conclusion Non-invasive CT larynx quantification of vocal cord movement was achieved. This new approach has identified frequent upper airway dysfunction in asthma with potential implications for disease control and treatment.

Supported by Monash Medical Centre, Emerging Researcher Fellowship Nomination Janet Elder International Travel.

Conflict of Interest No.
TP-037

INVESTIGATION AND CHARACTERIZATION OF PERSISTENT COUGH ASSOCIATED WITH H1N1 2009 INFLUENZA

NM RYAN ${ }^{1,2}$, AE VERTIGAN ${ }^{3}$, J FERGUSON ${ }^{4}$, P WARK ${ }^{1,2}$, PG GIBSON ${ }^{1,2,5}$ ${ }_{1}^{1}$ Priority Centre for Asthma \& Respiratory Diseases, University of Newcastle, ${ }^{2}$ Dept Respiratory \& Sleep Medicine, HMRI, John Hunter Hospital (JHH), Newcastle, ${ }^{3}$ Dept Speech Pathology, JHH, ${ }^{4}$ Hunter Area Pathology Service \& Division of Medicine $\mathrm{JHH}$, and ${ }^{5}$ Woolcock Institute of Medical Research, Sydney, Australia

Aim To investigate the characteristics and mechanisms of chronic cough (CC) following acute respiratory illness from laboratory-confirmed H1N1 2009 influenza.

Methods 136 subjects who had current symptoms and had been tested for H1N1 2009 influenza by PCR assay participated in this study. Twenty-one of those continued onto clinical testing. Investigations to assess cough included symptom questionnaires, hypertonic saline challenge and cough monitoring. Results Of the 136 participants, $43 \%$ tested positive for $\mathrm{H} 1 \mathrm{~N} 1$ and $57 \%$ tested negative for H1N1. H1N1-infected participants were younger and predominantly female. The prevalence of post-H1N1 CC was $9.4 \%$, and for non$\mathrm{H} 1 \mathrm{~N} 1$ infection, $4.9 \%$. Objectively measured cough frequency was 3 times greater; there was a 5-fold increase in cough reflex sensitivity, and greater quality-of-life impairment in the participants with chronic post-infectious cough than the non-cough participants.

Conclusions CC was found to be relatively common, mild in severity and tending to resolution with time. The characteristics of post-H1N1 CC were similar to other post-infectious cough and were associated with cough reflex hypersensitivity.

Supported by NHMRC CCRE in Respiratory and Sleep Medicine

Nomination Janet Elder International Travel Award.

Conflict of Interest No.

Journal Compilation @ 2011 Asian Pacific Society of Respirology

\section{ABNORMAL VOCAL CORD FUNCTION IN ACUTE ASTHMA EXACERBATIONS

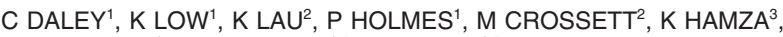 A GRAUDINS ${ }^{4}$, G HAMILTON $^{1,5}$, P BARDIN $^{1,5}$ \\ ${ }^{1}$ Respiratory \& Sleep Medicine, Monash Medical Centre, Vic., Australia, ${ }^{2}$ Diagnostic Imaging, Monash Medical Centre, Vic., Australia, ${ }^{3}$ Statistics, Monash University and Medical Centre, Vic., Australia, ${ }^{4}$ Accident and Emergency Department, Monash Medical Centre, Vic., Australia, and ${ }^{5}$ Monash Institute of Medical Research, Vic., Australia.}

Aim Upper airway dysfunction may accompany acute severe asthma, but this has not been investigated. A novel imaging technique, dynamic 320-slice computerized tomography (CT), was used to examine laryngeal behaviour in acute asthma exacerbation

Methods Patients were studied in the emergency department or as acute inpatients following admission for an acute exacerbation of asthma. Vocal cord movement was imaged by 320 -slice CT larynx and compared to normal vocal cord movement in a healthy cohort.

Results Vocal cord movement was abnormal with excessive narrowing during either inspiration, expiration or both in 11 of 16 cases $(68.7 \%)$ with acute severe asthma. Imaging again revealed that laryngeal dysfunction characterized the movement abnormality, rather than isolated vocal cord dysfunction. Radiation exposure was low and generally $<2$ milli-Sievert.

Conclusion Non-invasive CT larynx quantification of vocal cord movement was effectively achieved in acute severe asthma. We identified frequent upper airway dysfunction in acute severe asthma suggesting that treatment of upper airway obstruction (e.g. using BiPAP) may be merited during asthma exacerbation.

Supported by Nil.

Conflict of Interest No. 
CELL BIOLOGY/IMMUNOLOGY SIG: POSTER SESSION

TP-040

\section{EICOSANOIDS, DO THEY ALTER THE DEPOSITION OF THE EXTRACELLULAR MATRIX IN AIRWAY CELLS?}

D VAN LY, JK BURGESS, JL BLACK, BG OLIVER

The Woolcock Institute - Glebe, NSW, Australia

Aim To determine whether eicosanoids could alter the deposition of extracellular matrix (ECM) proteins and cytokine release from human airway cells. Methods Airway smooth muscle cells (ASM), fibroblasts and epithelial cells were stimulated with leukotrienes $B_{4}, C_{4}, D_{4}, E_{4}$ and the prostaglandins $E_{2}, D_{2}$, $F_{2 \alpha}$ and the $\mathrm{PGI}_{2}$ analogue MRE-269. After 72 hours, culture medium was collected and IL- 6 and IL-8 production and cell deposited ECM proteins tenascin C, fibronectin and perlecan were assessed by ELISA. To determine whether eicosanoids influenced cell proliferation, manual counting of cells in the experiments were carried out before and after stimulation.

Results Neither leukotrienes or prostanoids altered cell proliferation after 3 days of stimulation $(n>5)$. Leukotrienes had no effect on ECM protein deposition or cytokine release from ASM or fibroblasts $(n>5)$. Leukotrienes did not alter either parameters in epithelial cells except leukotriene D4, which increased tenascin C deposition $(n=7, p<0.05)$. Prostanoids induced IL-6 and IL-8 and other various changes in ASM and fibroblasts $(n>5, p<0.05)$ (see below).

\begin{tabular}{llclll}
\hline Cell & Parameter & PGE2 & PGD2 & PGF2a & MRE \\
\hline ASM & Fibronectin & $\downarrow$ & $\downarrow$ (at 10-5M) & - & $\downarrow$ \\
& Tenascin & - & $\downarrow$ (at 10-5M) & - & - \\
\multirow{5}{*}{ Fibroblasts } & Perlecan & $\downarrow$ & $\downarrow$ (at 10-5M) & N/A & - \\
& Fibronectin & $\downarrow$ & $\downarrow$ (at 10-5M) & - & $\downarrow$ \\
& Tenascin & $\downarrow$ & $\downarrow$ (at 10-5M) & - & $\downarrow$ (at 10-5M) \\
& Perlecan & $\downarrow$ & $\downarrow$ (at 10-5M) & N/A & N/A \\
\hline
\end{tabular}

Conclusion Leukotrienes alone did not affect the ECM proteins and cytokines assessed in this study. Prostanoids decreased ECM protein deposition whilst increasing cytokine release without affecting cell proliferation. This study shows that prostanoids may have a more pronounced role on direct ECM remodelling than leukotrienes in airway cells.

Supported by Merck.

Conflict of Interest No.

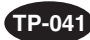

PRO-INFLAMMATORY CYTOKINE PRODUCTION IS IMPAIRED IN RESPONSE TO TLR2 ACTIVATION IN HEALTHY AGEING AND COPD

KM OREO ${ }^{1,2}$, KJ BAINES ${ }^{1,2}$, PG GIBSON ${ }^{1,2}$, PM HANSBRO', $^{1}$ JL SIMPSON1,2

${ }^{1}$ Centre for Asthma and Respiratory Disease, The University of Newcastle, NSW, and ${ }^{2}$ Department of Respiratory and Sleep Medicine, Hunter Medical Research Institute, NSW

Background Toll-like receptor (TLR)2 is an innate immune receptor involved in the initial detection of pathogen-associated molecular patterns. The effect of ageing and chronic obstructive pulmonary disease (COPD) on TLR2 responses and the impact of these innate immune responses in COPD pathogenesis remain unclear.

Hypothesis Expression and activity of TLR2 on peripheral blood mononuclear cells (PBMCs) is increased with healthy ageing and further increased in COPD.

Methods PBMCs from healthy controls $<55$ years and $>55$ years; and participants with COPD ( $n=8$ per group) were cultured with or without Pam3C YSK4 (TLR2 agonist). Cells and supernatants were collected at 24 hours and protein (cytometric bead array or flow cytometry) and gene (real time PCR) expression was examined.

Results TLR2 activation led to increased release of interleukin (IL)-8, 6, $1 \beta$, and tumor necrosis factor (TNF)- $\alpha$. TLR2 gene expression was increased with stimulation; however, cell surface receptor levels were unchanged. There was no difference in the level of TLR2 between the 3 groups. In older people, TLR2 activation resulted in less IL-1 $\beta$ and TNF- $\alpha$ release, but similar release of IL-8 and IL-6. Similar results were seen in COPD. At baseline in COPD, there was up-regulation of TNF- $\alpha$ gene expression compared to the older healthy group; however, the TLR2 cytokine response did not differ between the groups.

Conclusion Healthy ageing is characterized by an impaired systemic proinflammatory cytokine response to TLR2-mediated innate immune activation. This effect persists in COPD and is selective in the cytokine pathways involved. These altered inflammatory mechanisms may affect responses to infection and injury impacting disease pathogenesis and warrant further evaluation.

Supported by NHMRC.

Conflict of Interest No.
AIRWAY EPITHELIUM BARRIER INTEGRITY COMPROMISATION FOLLOWING RHINOVIRAL INSULT

$\mathrm{KLOOI}^{1,2}, \mathrm{KM} \mathrm{LING}^{2,3}, \mathrm{EN}$ SUTANTO 2,3, A LARCOMBE $^{3}, \mathrm{R} \mathrm{FOONG}^{3}$, P RIGBY ${ }^{4}$, SM STICK ${ }^{1,2,3}$, A KICIC $\mathrm{KI}^{1,2,3}$

${ }^{1}$ School of Paediatrics and Child Health, UWA, WA, ${ }^{2}$ Department of Respiratory Medicine, PMH, WA $,{ }^{3} \mathrm{TICHR}, \mathrm{WA},{ }^{4}$ Centre for Microscopy, Characterization and Analysis, UWA, WA

Introduction The function of asthmatic airway epithelium is disrupted facilitating immune and inflammatory responses resulting in epithelial damage. Human rhinovirus (HRV) causes asthma exacerbations in children; however, paucity exists on how it affects barrier function. This study assessed how HRV infection affects epithelial barrier function and integrity in healthy and asthmatic epithelium.

Methods Adult BALB/c mice were intranasally infected with HRV-1B and followed for 21 days. Tight junction (TJ) expression was assessed using immunohistochemistry $(\mathrm{IHC})$ and Western blot analysis. Primary airway epithelial cells from healthy and asthmatic children were assessed for TJ gene and protein expression by qPCR and IHC, respectively.

Results Occludin and zonal occludin-1 (ZO-1) expression was lost and sustained in mice infected with HRV-1B however was not observed in shaminjected mice. Asthmatic airway epithelial cells were found to exhibit elevated basal gene expression levels of TJs (ZO-1, occludin and plakophilin-3 (PKP-3)) but markedly lower corresponding protein levels.

Conclusion HRV-1B compromises barrier function in vivo through sustained loss of TJ proteins. The marked decreased expression of TJ proteins in paediatric asthmatic epithelium may contribute towards increased susceptibility to viral infections. Disparity between gene and protein TJ expression could indicate either post-transcriptional regulation or compensatory effects by other TJ proteins and requires further study.

Supported by Asthma Foundation WA; NHMRC

Conflict of Interest None.
DO INNATE INTERFERONS MODIFY ADAPTIVE IMMUNITY TO RHINOVIRUSES?

JW UPHAM, ML CARROLL, AL PRITCHARD

The University of Queensland School of Medicine, and Department of Respiratory Medicine, Princess Alexandra Hospital, Brisbane, Australia

Background Rhinoviruses (RV) play an important role in acute exacerbations of various respiratory diseases, and this may be related to impaired immunity. Surprisingly, little is known about the determinants of adaptive recall responses to RV in healthy people. This study sought to determine the role of type I and type III interferons and plasmacytoid dendritic cells (pDC) in regulating this process.

Methods Blood mononuclear cells from 26 healthy people were cultured with RV16 for up to 5 days. Cytokines were measured by qPCR and ELISA.

Results RV16 induced robust expression of IFN $\alpha$, IFN $\beta$ and IFN-stimulated genes at early time points, but this was not statistically associated with adaptive immune responses at day 5 (IFN $\gamma$ and IL-13). Blocking endogenous IFNo and IFN $\beta$ with the decoy receptor B18R did not reduce IFN $\gamma$ and IL-13 synthesis, while addition of recombinant IFN $\beta$ or IFN $\lambda 1$ actually inhibited IFN $\gamma$ and IL-13 synthesis. Depletion of pDC resulted in significant reductions of the chemokine IP-10 at 24 hours, but no change in the adaptive cytokines at day 5.

Conclusions RV stimulated IFN $\gamma$ and IL-13 synthesis appears to proceed independently of type I and type III interferons and pDC. Studies in progress are examining alternative methods to augment adaptive immunity to rhinoviruses.

Support The Asthma Foundation of QLD, NHMRC.

Conflict of Interest None.

Journal Compilation @ 2011 Asian Pacific Society of Respirology 
OVER-EXPRESSION OF THE GRANZYME B INHIBITOR, PI-9 AS AN IMMUNE-EVASION MECHANISM IN LUNG CANCER

CCS SORIANO 1,2 , VR MUKARO', G HODGE $1,2,3$, M HOLMES ${ }^{2,3}$, H JERMANN ${ }^{1,2,3}$, D MOFFAT ${ }^{4}$, D MEREDITH ${ }^{4}$, C JURISEVIC ${ }^{5}$, PN REYNOLDS ${ }^{1,2,3}$, S HODGE ${ }^{1,2,3}$

${ }^{1}$ Lung Research Laboratory, Hanson Institute, ${ }^{2}$ University of Adelaide, Adelaide South Australia, ${ }^{3}$ Department of Thoracic Medicine and ${ }^{5}$ Cardiothoracic Surgery, Royal Adelaide Hospital, ${ }^{4}$ Department of Surgical Pathology, SA Pathology

COPD patients have an increased risk of developing lung cancer. $C D 8^{+}$cytotoxic T-cells mount immune responses to cancer via cytotoxic pathways including granzyme B (GrB). However, cancer cells are known to develop immune evasion mechanisms. We therefore hypothesized that lung cancer cells would over-express the GrB-inhibitor, proteinase inhibitor-9 (PI-9) and down-regulate GrB expression by $\mathrm{CD}^{+} \mathrm{T}$-cells as an immune evasion mechanism.

Methods PI-9 expression in primary lung cancer epithelial cells and lung cancer cell lines was assessed using flow cytometry and immunohistochemistry. GrB and PI-9 expression by $\mathrm{CD}^{+}{ }^{+}$-cells in the airway (BAL) and lung tissue/tumour were investigated in subjects with lung cancer and/or COPD and Controls by flow cytometry.

Results In primary lung cancer cells, there was significantly higher $\mathrm{PI}-9$ expression (mean (SEM)) (57.4 \% (3.1)) compared to controls $(27.3 \%(4.9))$. Immunohistochemical staining confirmed these results. Significantly increased $\mathrm{PI}-9$ expression was noted in various lung cancer cell lines, with the more aggressive small cell cancer (SBC-1: $59.2 \%$ (3.6), SBC-3: 81.4\% 93.5)) and the non-small cell lung carcinoma subtype $\mathrm{NCl}-\mathrm{H} 2009$ (68.8\% (12.9)) showing the highest expression versus a control (BEAS-2B cell line $16.7 \%(6.1)$ and primary large airway epithelial cells $24.1 \%$ (18.2)). There was decreased CTL $\mathrm{GrB}$ expression in cancer tissue-derived $\mathrm{CD}^{+} \mathrm{T}$-cells versus non-cancer tissue-derived $C D 8^{+} \mathrm{T}$-cells in patients with COPD versus controls.

Conclusions $\mathrm{Pl}-9$ over-expression by cancer cells and down-regulation of $\mathrm{GrB}$ by lung cancer-associated $\mathrm{CD} 8^{+} \mathrm{T}$-cells suggest an immune-evasion mechanism against GrB-mediated cytotoxicity in lung cancer.

Conflict of Interest None.

\section{MATRIX METALLOPROTEINASE-2 (MMP-2) REGULATES MIGRATION OF TSC2-NULL MOUSE EMBRYONIC FIBROBLASTS}

H YIN NG ${ }^{1,2,3}$, B GG OLIVER ${ }^{1,2,3}$, JK BURGESS ${ }^{1,2,3}$, VP KRYMSKAYA ${ }^{4}$, JL BLACK ${ }^{1,2,3}$, LM MOIR ${ }^{1,2,3}$

${ }^{1}$ Discipline of Pharmacology, The University of Sydney, NSW, ${ }^{2}$ Cell Biology, Woolcock Institute of Medical Research, NSW, ${ }^{3}$ Cooperative Research

Centre for Asthma and Airways, NSW, and ${ }^{4}$ Department of Medicine, University of Pennsylvania, Philadelphia, PA, USA

Aim To investigate whether the inhibition of matrix metalloproteinase-2 (MMP-2) by a non-selective MMP inhibitor (doxycycline) and the specific MMP-2 inhibitor I (olic acid) can regulate cellular migration of TSC2-null mouse embryonic fibroblasts (MEFs), which act as a model for lymphangioleiomyomatosis (LAM) cells, as compared to wild-type MEFs.

Methods Wild-type (TSC2-positive) and TSC2-null MEFs were treated with diluent, doxycycline $(0.1 \mathrm{pg} / \mathrm{mL}-100 \mu \mathrm{g} / \mathrm{mL})$ or olic acid $(0.3-30 \mu \mathrm{M})$ for 24 hours. MMP-2 levels were assessed by zymography and ELISA. Cell migration for 4 hours was measured using a transwell migration assay.

Results Under basal conditions, MMP-2 release and cellular migration was 2.3-fold and 2.9-fold higher, respectively, in TSC2-null MEFs compared to TSC2-positive MEFs (MMP-2 release, TSC2-null $(n=6)$ and TSC2-positive ( $n$ $=6), p<0.05$; cell migration, TSC2-null $(n=10)$ and TSC2-positive $(n=10)$, $\mathrm{p}<0.05$ ). MMP-2 release was reduced in TSC2-null MEFs after 24-hour treatment with doxycycline $(30$ and $100 \mu \mathrm{g} / \mathrm{mL}, \mathrm{n}=6, \mathrm{p}<0.05)$ and with olic acid $(1-30 \mu \mathrm{M}, \mathrm{n}=3, \mathrm{p}<0.05)$. Treatment with doxycycline $(10 \mathrm{pg} / \mathrm{mL}-100 \mu \mathrm{g} / \mathrm{mL}$, $\mathrm{n}=5, \mathrm{p}<0.05)$ or olic acid $(1-30 \mu \mathrm{M}, \mathrm{n}=3, \mathrm{p}<0.05)$ also significantly reduced cell migration of TSC2-null MEFs.

Conclusions The loss of TSC2 up-regulates MMP-2 production that results in increased cell migration. Inhibition of MMP, more specifically MMP-2, may prove to be beneficial in the treatment of LAM.

Supported by LAM Australasia Research Alliance (LARA), CRC for Asthma and Airways, The New Zealand LAM Charitable Trust.

Conflict of Interest No.

\section{EXPRESSION PROFILE OF THE SPHINGOSINE KINASE} SIGNALLING SYSTEM IN THE LUNG OF PATIENTS WITH COPD

F CORDTS $^{1,5,6}$, S PITSON ${ }^{2}$, I GIBBINS ${ }^{1}$, D MOFFAT ${ }^{3}$, D MEREDITH ${ }^{4}$, C JURISEVIC ${ }^{5}$, H JERSMANN ${ }^{5,6}, S^{2}$ HODGE ${ }^{5,6}$, R HABERBERGER ${ }^{1}$

${ }^{1}$ Centre for Neuroscience Flinders Medical Science \& Technology, Flinders University, ${ }^{2}$ Centre for Cancer Biology, and ${ }^{3}$ Department of Surgical

Pathology, SA Pathology, ${ }^{4}$ Cardiothoracic Surgery, Royal Adelaide Hospital, ${ }^{5}$ Lung Research, Hanson Institute, ${ }^{6}$ University of Adelaide, Adelaide South Australia

COPD is a leading cause of death worldwide. Treatments are limited and restricted to symptomatic care. There is an urgent need for new treatment options targeting the inflammation. Tissue damage in COPD is thought to result from an inability of the normal repair processes with accumulation of apoptotic material and impaired clearance of this material by macrophages in the airways. Lung inflammation and macrophage function involves the bioactive sphingolipid sphingosine 1-phosphate (S1P). Multiple studies have showed the involvement of these components in inflammation.

Methods We investigated lung tissue samples from 55 patients (COPD or non COPD controls) undergoing curative lobectomy for lung cancer. We analysed the mRNA expression profile, the sphingosine-kinase (SphK) protein activity and the localization and expression of individual proteins.

Results We show in this study for the first time a comprehensive expression profile of all synthesizing enzymes, receptors and degrading enzymes in the human lung. Correlations between receptor subtypes, degrading enzymes and between S1P receptor subtype 1 were detected. Multivariance ANOVA showed that in COPD, the relative mRNA expression of S1P receptor subtype 5 was reduced.

Conclusion The correlations between receptors and enzymes involved in the sphingosine kinase signalling system in the lung suggest common regulatory mechanisms. S1PR5 is expressed on dendritic and NK cells which are reduced under conditions of COPD. Therefore, our findings of reduced S1PR5 in COPD may provide a novel target for pharmacotherapy.

Support Flinders Medical Centre Foundation.

Conflict of Interest Nil.

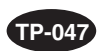

LUNG CANCER CELLS INHIBIT THE PHAGOCYTIC FUNCTION OF ALVEOLAR MACROPHAGE AND U937 CELLS

\section{FC DEHLE ${ }^{1}$, E RANIER ${ }^{2}$, G HODGE ${ }^{1}$, S MISKOVICH ${ }^{1}$, PN REYNOLDS ${ }^{1}$,} $S$ HODGE

${ }^{1}$ Department of Thoracic Medicine, Royal Adelaide Hospital, and Lung Research Laboratory, Hanson Institute, and ${ }^{2}$ SA Neonatal Screening Centre, SA Pathology, Adelaide, South Australia

Lung cancer is responsible for more cancer-related deaths than colon, breast and prostate cancers combined. In patients with COPD and/or lung cancer, we have shown a reduction in lung and airway macrophage function, evident by a reduced ability to phagocytose apoptotic airway epithelial cells and neutrophils. The potential for lung cancer cells to directly inhibit this function (a potential immune evasion mechanism) has not been investigated.

Methods We investigated whether soluble mediators produced by lung cancer cells suppressed macrophage phagocytic function. We harvested 48 hours supernatant from human lung adenocarcinoma (H2009, H1466) and the small cell lung carcinoma (SBC1) cell lines. Alveolar macrophages (AM) were collected from BAL from 4 controls, and 8 subjects with lung cancer. We also investigated the effects on PMA-differentiated U937 macrophage-like cells. AM or U937 cells were treated with cancer cell line supernatants for 24 hours. The cells were then assessed for their ability to phagocytose apoptotic cells and their expression of recognition molecules. To investigate soluble mediators produced by the lung cancer cells, we measured prostanoids, isoprostanes and lipoxygenase by HPLC tandem.

Results Cancer cell supernatant decreased the ability of AM from controls to phagocytose apoptotic cells (\% decrease: SBC 33\%, $p=0.034 ; \mathrm{H} 200917 \%$, $p=0.11 ; \mathrm{H} 146629 \%, p=0.18)$. This was associated with a decrease in expression of complement receptors- 3 and -4 . SBC1 and $\mathrm{H} 2009$ supernatants also significantly reduced phagocytosis in U 937 cells by $58 \%$ and $54 \%$, respectively. There were no significant effects on AM from cancer patients. Changes identified by mass spectrometry in the lung cancer cells included PGE2, PGD2, PGF2a, TXB2 DHA, EPA and AA.

Conclusion A potential immune evasion mechanism in lung cancer may be the inhibition of macrophage phagocytic function via release of soluble mediators by the lung cancer cells.

Supported by ARC.

Conflict of Interest No. 
TP-048

\section{ACTIVATION OF THE PLASMA KALLIKREIN-KININ SYSTEM ON} PLEURAL MESOTHELIAL CELLS

\author{
JF VARANO DELLA VERGILIANA ${ }^{1}$, S LANSLEY ${ }^{2}$, AL TAN ${ }^{2}$, J CREANEY ${ }^{3}$, \\ YCG LEF 2,4 GA STEWART \\ ${ }^{1}$ School of Biomedical, Biomolecular and Chemical Sciences, University of \\ Western Australia, Perth, WA, ${ }^{2}$ Centre for Asthma, Allergy and Respiratory \\ Research, School of Medicine \& Pharmacology, University of Western \\ Australia, Perth, WA, ${ }^{3}$ National Research Centre for Asbestos Related \\ Diseases, School of Medicine \& Pharmacology, University of Western \\ Australia, Perth, WA, and ${ }^{4}$ Respiratory Dept, Sir Charles Gairdner Hospital, \\ Perth, WA
}

Aim To determine if bradykinin, the end product of plasma kallikrein-kinin system (KKS) activation, is present in pleural effusions and whether mesothelial cells can efficiently activate the plasma KKS.

Methods Pleural effusions were obtained from patients with a range of common pleural etiologies and bradykinin concentrations determined by ELISA. Plasma kallikrein formation and bradykinin release were used to assess plasma KKS activation on mesothelial cells. Calcium mobilization was examined by flow cytometry.

Results Pleural fluid bradykinin concentrations did not correlate with disease diagnoses but were elevated in exudative effusions. Pleural mesothelial and mesothelioma cells assembled components of the plasma KKS to form plasma kallikrein and liberate bradykinin in a process dependent on heat shock protein 90. Calcium mobilization was induced in mesothelium-derived cell lines by bradykinin, but not des-arg ${ }^{9}$-bradykinin, confirming downstream actions of kinin formation are likely mediated via the bradykinin receptor subtype 2 .

Conclusions Pleural mesothelial cells support activation of the plasma KKS by a mechanism dependent on HSP90, and may contribute to KKS-mediated inflammation in pleural disease. This may present a potential target for antiinflammatory therapies in serosal inflammation.

Supported by Australian NHMRC, Asthma Foundation of WA.

Conflict of Interest None.

\section{MIRNA-222 AND MIRNA-223 REGULATE MALIGNANT MESOTHELIOMA CELL MIGRATION}

KA BIRNIE, B BADRIAN, SE MUTSAERS

Lung Institute of Western Australia, WA, Australia

Background Malignant mesothelioma (MM) is an aggressive cancer associated with asbestos exposure. MM has a poor prognosis, and currently, there are only limited therapies and treatments for this disease. A popular topic of cancer research includes the role played by MicroRNAs (miRNAs). miRNAs are a family of 21-25 nucleotide single-stranded RNAs which cause sequencespecific post-transcritpional repression to regulate gene expression.

Aim To test the hypothesis that miRNA-223 and miRNA-222 are involved in regulating cell proliferation and motility in MM.

Methods The expression of miRNAs 222 and 223 and their targets (stathmin and $\mathrm{p} 27^{\mathrm{kip} 1}$ ) mRNA was determined using real-time PCR in human and mouse MM cell lines and control mesothelial cells. Stathmin and p2 $7^{\text {kip } 1}$ protein levels were measured by Western blots. The levels of miR-223 and miR-222 were up- or down-regulated within the cells by transient transfection of over-expression and inhibitor constructs. The functional role that these miRNAs have in MM was determined by investigating cell proliferation and motility.

Results miR-223 was expressed at significantly lower levels $(p \leq 0.01)$ in the MM cells compared to control mesothelial cells. In the same cell lines, stathmin RNA and protein were expressed significantly higher $(p \leq 0.05)$. A strong inverse correlation between the levels of miR-223 and stathmin existed. The expression of miR-222 and p27 $7^{\mathrm{kip} 1}$ was variable between the cell lines; however, an inverse correlation was shown between miR-222 and p2 $7^{\text {kip } 1}$ levels. Following over-expression of miR-223, stathmin mRNA and protein expression decreased, confirming miR-223 targets and regulates stathmin in MM. Similarly, inhibiting miR-222 caused an increase in the expression of $\mathrm{p} 27^{\mathrm{kip} 1}$ confirming miR-222 targets and regulates $\mathrm{p} 27^{\mathrm{kip} 1}$ in MM. Over-expressing miR-223 and inhibiting miR-222 did not alter cell proliferation but did cause a decrease in cell migration.

Conclusions miR-223 and miR-222 may play role in regulating cell migration in MM. This is potentially due to their interactions with stathmin and p27 $7^{\mathrm{kip} 1}$. Nomination Nil.

Conflict of Interest No.

\section{TP-049}

\section{IDENTIFICATION AND CHARACTERIZATION OF A NOVEL}

\section{SPLICE VARIANT OF HUMAN KININ B 1 RECEPTOR}

FY CHEAH ${ }^{1,2}, \mathrm{KD}_{\mathrm{BHOOLA}}^{2,3}$, PJ THOMPSON $\mathrm{N}^{1,2,3}$

${ }^{1}$ Cooperative Research Centre for Asthma and Airways, ${ }^{2}$ School of Medicine and Pharmacology, University of Western Australia, Nedlands, WA, and ${ }^{3}$ Lung Institute of Western Australia

Background Kinins have been implicated in airway lung diseases such as asthma and lung cancer by regulating inflammation, cell proliferation and migration. The effect of kinins is mediated through the binding of two receptors, kinin $B_{1}$ and $B_{2}$ receptors $\left(B_{1} R\right.$ and $\left.B_{2} R\right)$. A novel $B_{1} R$ splice variant (SV) resulting in a shorter $5^{\prime}$ untranslated region (UTR) was identified in cultured airway epithelial and fibroblasts as well as in lung carcinoma tissue and leukocytes. This study aims to characterize the functional role of the novel $B_{1} R$ SV in mRNA stability, translation efficiency and receptor expression in cultured airway epithelial cells.

Methods Stability of $B_{1} R$ SV was determined by measuring $B_{1} R$ mRNA levels over time in $\mathrm{H} 2126$ cells after actinomycin D treatment. Translational efficiency of WT and SV 5'UTR was determined by measuring luciferase activity in transfected H2126 cells. Expression of WT and SV transcripts through Q-RTPCR were compared in cells treated with a $B_{1} R$-specific agonist DAKD. Cell-surface receptor expression post-agonist stimulation was quantified using FACS.

Results mRNA stability studies indicated that $B_{1} R$ SV was $\approx 35 \%$ less stable than the WT transcript in H2126 cells suggesting a stabilizing element 5'UTR. Translation efficiency of SV was no different to WT $B_{1} R$. DAKD stimulation increased both WT and SV transcripts early in the time course, although the peak expression of WT and SV differed at 3 hours and 6 hours, respectively. DAKD stimulated cells showed two phases of receptor expression, (1) decrease of cell surface receptor up to 4.5 hours post-stimulation; (2) increase in cell surface $B_{1} R$ after 4.5 hours.

Conclusion This study has identified a novel regulatory mechanism of $B_{1} R$ expression through the production of a SV that alters the 5'UTR. The translation efficiency of $B_{1} R$ is not affected, but the SV was less stable than the WT in $\mathrm{H} 2126$ cells and may play a role in allowing quicker changes in transcription. Agonist-induced up-regulation of transcripts in a time-dependent manner may be important in maintaining a chronic response during inflammation.

Conflict of Interest No.

\section{CHARACTERISTICS OF CHYLOUS FLUID FROM LYMPHANGIOLEIOMYOMATOSIS (LAM) PATIENTS}

LM MOIR $^{1,2}$, M WECKMANN $^{1,2}$, JL BLACK $^{1,2}$

${ }^{1}$ Woolcock Institute of Medical Research, NSW 2037, and ${ }^{2}$ Discipline of Pharmacology, The University of Sydney, NSW, 2006, Australia

Rationale Lymphangioleiomyomatosis (LAM) is a rare and progressive disease affecting predominantly females of childbearing age and for which there is no current treatment. The pathological characteristics of LAM include lesions of smooth muscle-like cells (LAM cells) and an abundance of lymphatic vessels within the lungs. Increased lung lymphangiogenesis is associated with decreased survival rates and chylothorax occurs in $20-40 \%$ of LAM patients. Objective The aim of this study was to investigate the properties of chylous fluid from LAM patients.

Methods Three samples of chylous fluid were obtained from two LAM patients.

Chylous fluid supernatant was assayed for the presence of a range of cytokines, angiogenic proteins and growth factors using ELISA and protein arrays. Chylous-derived cells were cultured and proliferation assessed using the MTT assay.

Results The lymphangiogenic factor VEGF-D was present in chylous fluid $(1665 \pm 332 \mathrm{pg} / \mathrm{mL}, \mathrm{n}=3)$. A range of other proteins including MIF, IP-10, Serpin E1, angiogenin, MMP-9, TIMP-1, TIMP-4, prolactin and VEGF was also detected. However, levels of VEGF-C and PDGF-BB were not detected in chylous fluid. Treatment of chylous-derived cells with chylous fluid induced proliferation.

Conclusion Chylous fluid contains a vast array of proteins which may contribute to the increased lymphangiogenesis associated with LAM.

Supported by LAM Australasia Research Alliance (LARA), Dr Allan Glanville and Ros Pollard.

Conflict of Interest No.

Journal Compilation @ 2011 Asian Pacific Society of Respirology 
DOES BETA2 ADRENOCEPTOR (ADRB2) DENSITY VARY IN CIRCULATING LYMPHOCYTES COMPARED WITH MONOCYTES?

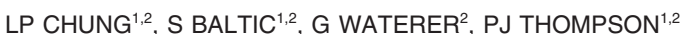 \\ ${ }^{1}$ Lung Institute of Western Australia (Inc) \& CRC Asthma and Airways, and \\ ${ }^{2}$ School of Medicine and Pharmacology, University of Western Australia
}

Circulating lymphocytes are increasingly used as a surrogate cell type to reflect changes in ADR $\beta 2$ density elsewhere in the body, particularly the respiratory system. However, ADR 32 density is non-uniform among lymphocyte subsets and it is unclear if, and the degree to which, ADR $\beta 2$ density varies between individuals.

Aim To assess the extent of variability in ADR $\beta 2$ density on human peripheral blood mononuclear cells (PBMC) including lymphocytes and monocytes. Method PBMC were isolated from blood of 10 healthy subjects by density gradient centrifugation with Ficoll-Paque. Cell surface and total ADR $\beta 2$ of intact and permeabilized lymphocytes (CD14+) and monocytes (CD3+) were measured using anti-ADR $\beta 2$ via FACS. Geometric mean fluorescence (GMF) was used as the indices for ADR $\beta 2$ density per cell.

Result Surface ADRB2 - GMF increased by 3.54- and 4.62-folds over negative controls for lymphocytes and monocytes, respectively. Magnitude of foldchange was not significantly different between these cells $(p=0.16)$, but the distribution of GMF intensity between samples suggests greater variability in ADRß2 density in lymphocytes versus monocytes $(p=0.06)$. Proportion of cells-stained ADRB2-positive was significantly higher in monocytes versus lymphocytes $(71.9 \pm 3.6 \%$ vs. $36.7 \pm 8.5 \%, \mathrm{p}=0.02)$. Total $A D R \beta 2-\mathrm{GMF}$ increased by $12.4 \pm 5.8$ and $9.61 \pm 3.8$-folds for lymphocytes and monocytes, respectively $(p>0.05)$. Proportion of ADR $\beta 2$-positively stained cells were similar between samples (lymphocytes $80 \%$, monocytes $86 \%, p=0.47$ ), but greater variability was observed for lymphocytes (range 27-99\%) versus monocytes (66-100\%).

Conclusions Despite similarities in surface and total ADR $\beta 2$ density, lymphocytes display greater inter-subject variability compared with monocytes. This will have implication in experimental designs and interpretation of changes in ADR $\beta 2$ density in studies using human PBMC as an alternative to primary cells from the organ of interest.

Conflict of Interest No.

\section{THE INTERPLAY BETWEEN PGE2, IL-1 $\beta$ AND HUMAN} AIRWAY EPITHELIUM

S BALTIC, I RANAWEERA, MP DUNN, SEL TEMPLE, PJ THOMPSON

Lung Institute of Western Australia, University of Western Australia,

Perth, WA

PGE2 plays a protective role in asthma by inhibiting airway inflammation. It is predominantly produced by epithelial cells in response to pro-inflammatory stimuli and acts as an autocrine and paracrine mediator. On the contrary, IL-1B is a highly potent cytokine that induces many pro-inflammatory effects in the human airway including activation of the human lung epithelium which promotes production of pro-inflammatory cytokines and chemokines. Airway epithelial cells express all four known PGE2 (E Prostanoid (EP) receptors, but mechanisms underlying the regulation of expression of EP receptors in human lung epithelial cells have remained elusive. Therefore, we investigated whether PGE2, an endogenous protective mechanism of the airways, can modulate IL-1 $\beta$ influence on EP receptor expression in human epithelial cells.

Methods EP receptor mRNA and protein expression was quantified in 16-HBE cells at basal levels and following stimulation with IL-1 $\beta$ or PGE2 alone, or simultaneously, using Real Time RT PCR and FACS analysis, respectively.

Results PGE2 up-regulates all four EP receptors at mRNA level, while IL-1B up-regulates EP1, EP2 and EP4 and does not influence expression of EP3. At protein level, preliminary results show transient increase of EP1 receptors in the presence of PGE2, while IL-1 1 down-regulates this receptor. EP2 and EP4 are up-regulated following stimulation with both stimuli. Importantly, antiinflammatory EP3 receptor is up-regulated only in the presence of PGE2.

Conclusion We show for the first time that PGE2 may influence expression of its own receptors and oppose the effect of IL-1 $\beta$ in human lung epithelial cells. This may in turn alter PGE2 production and autocrine activation with potential implication on the function of epithelial cells, which is important in modulation of immune response in asthma and lung inflammatory diseases. Nomination Nil.

Conflict of Interest No.

Journal Compilation (C) 2011 Asian Pacific Society of Respirology

\section{COPD SIG: POSTER SESSION 1}

\section{COPD IN AUSTRALIA: AN UPDATE ON THE NATIONAL TRENDS}

MR GALL, AL WEBSTER

Australian Institute of Health and Welfare, Canberra, ACT

The epidemiology of COPD at a national level has been analysed to provide an update of the key trends and macro-level factors influencing COPD in Australia, including gender-based comparisons and analysis of disparities between sub-populations.

Methods National-level datasets were analysed including the National Health Survey, National Hospital Morbidity Database and the National Mortality Database. A variety of cross-sectional and trend-based analytical techniques were used to identify relevant factors.

Results The results include clear gender effects and important variations between sub-populations, including effects of remoteness and indigenous and non-indigenous status.

Conclusions While the overall mortality and morbidity related to COPD in Australia is declining, these changes are not uniform across the population. Supported by Australian Government Department of Health and Ageing. Conflict of Interest 
TP-056

PREVALENCE OF RESPIRATORY SYMPTOMS, ILLNESSES AND SPIROMETRIC DIAGNOSES IN THE AUSTRALIAN BOLD STUDY

W XUAN ${ }^{1}$, B TOELLE ${ }^{1}$, T BIRD $^{1}$, M ABRAMSON $^{2}$, B GRAHAM $^{3}$, A JAMES $^{4}$, D JOHNS ${ }^{5}$, G MAGUIRE ${ }^{6}, \mathrm{R}^{2}$ WOOD-BAKER ${ }^{5}$, G MARKS

${ }^{1}$ Woolcock Institute of Medical Research, NSW, ${ }^{2}$ Monash University,

Vic., ${ }^{3}$ Charles Sturt University, NSW, ${ }^{4}$ Sir Charles Gairdner Hospital, WA,

${ }^{5}$ Menzies Research Institute, TAS, and ${ }^{6}$ James Cook University, QId

The Burden of Obstructive Lung Disease (BOLD) Study is an international study designed to measure the prevalence, risk factors and burden of COPD. Data collection using the BOLD protocol has been undertaken at eight sites with inclusion of urban, rural, coastal and inland regions of Australia. Methods A random sample of adults aged $\geq 40$ years was identified. Information on respiratory symptoms and diagnosed COPD were collected by questionnaire. Post-bronchodilator FEV ${ }_{1}$ and FVC were used to define GOLD stage. The (un-weighted) prevalence rates are presented by age groups and sex. Results

\begin{tabular}{|c|c|c|c|c|c|c|}
\hline \multirow[b]{2}{*}{ Prevalence (\%) } & \multicolumn{2}{|c|}{$40-54$ years } & \multicolumn{2}{|c|}{$55-74$ years } & \multicolumn{2}{|c|}{$75+$} \\
\hline & M & $\mathrm{F}$ & M & $\mathrm{F}$ & M & $\mathrm{F}$ \\
\hline Cough on most days & 11.2 & 10.3 & 9.5 & 11.2 & 11.7 & 10.1 \\
\hline Wheeze in the last 12 months & 30.2 & 32.4 & 29.2 & 29.3 & 25.9 & 21.7 \\
\hline Ever diagnosed with COPD & 3.3 & 6.7 & 8.7 & 8.2 & 11.7 & 9.3 \\
\hline GOLD stage $1+$ & 8.3 & 6.3 & 21.3 & 14.9 & 41.6 & 31.0 \\
\hline GOLD stage $2+$ & 4.7 & 3.2 & 10.6 & 8.8 & 19.9 & 16.3 \\
\hline $\begin{array}{l}\mathrm{BD} \text { reversibility }\left(\mathrm{FEV}_{1} \geq 12 \% \text { of }\right. \\
\text { pre-bronchodilator and } \geq 200 \mathrm{~mL} \text { ) }\end{array}$ & 5.3 & 3.8 & 6.5 & 3.6 & 5.6 & 4.7 \\
\hline Ever smoked & 55.3 & 57.1 & 60.1 & 41.2 & 61.7 & 34.9 \\
\hline
\end{tabular}

Conclusions Self-reported wheeze in the last 12 months is very common in adults over 40 years. In the younger age group (40-54 years), many people with wheeze did not have airflow obstruction or reversible spirometry at the time of test.

Support National Health and Medical Research Council.

Conflict of Interest No.

ASSOCIATION BETWEEN CHANGE OF FEV 1 AMONG COPD PATIENTS AND AMBIENT ULTRAFINE PARTICLE NUMBER CONCENTRATIONS

TLJC SIRITUNGA ${ }^{1,2}$, A LEWIS $^{3}$, M DENNEKAMP $^{1}$, MJ ABRAMSON ${ }^{1}$

${ }^{1}$ School of Public Health \& Preventive Medicine, Monash University,

Melbourne, ${ }^{2}$ Ministry of Health, Sri Lanka, and ${ }^{3}$ Dept of Allergy, Immunology \& Respiratory Medicine, The Alfred, Melbourne, Vic.

Aim To determine whether there is any association between change in FEV among COPD patients and ambient ultrafine particle number concentrations in Melbourne.

Methods Participants with mild to moderate COPD were asked to measure their FEV 1 using a portable electronic spirometer (PiKO) two times a day (morning and evening) for consecutive 7 days. The same procedure was repeated on average 9 months later. Ambient ultrafine (diameter $<0.1 \mu \mathrm{m}$ ) particle number concentrations were measured for the same period using an ultrafine condensation particle counter and Micro-Orifice Uniform Deposit Impactor.

Results 108 participants (mean (SD) age 66.2 (7.6) years, 72 males and 36

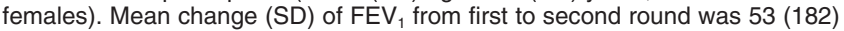
$\mathrm{mL}$. Mean change (SD) of the ultrafine particulate number concentration for the same period was $1096(3953) \mathrm{cm}^{-3}$. There were positive associations between $\mathrm{FEV}_{1}$ change and age (regression coefficient $4.39,95 \% \mathrm{Cl}-0.2$ to 8.97), smoking status $(2.82,-54.35$ to 60.0$)$ and male sex $(2.89,-77.3$ to 71.51). There was a weak association between decreasing FEV change and $_{1}$ increasing ultrafine particle concentration change $(-0.005,-0.014$ to 0.003$)$. None of these achieved statistical significance.

Conclusions The negative results are probably due to lack of power from the small sample size, low ultrafine concentrations, participants with normal lung function and lack of considerations of other pollutants.

Supported by NHMRC.

Nomination No.

Conflict of Interest Nil.
TP-058

\section{THE RELATIONSHIP BETWEEN EMPHYSEMA EXTENT AND VENTILATION HETEROGENEITY}

S TIMMINS ${ }^{1,2,3,4}$, G KING ${ }^{1,2,3,4}$, C SALOME ${ }^{1,3,4}$, R SCHOEFFEL $^{1,2,3}$, C WALSH ${ }^{1,3,4}$, N BEREND $^{1,3,4}$

${ }^{1}$ Woolcock Institute of Medical Research NSW, ${ }^{2}$ Department of Respiratory Medicine Royal North Shore Hospital NSW, ${ }^{3}$ Department of Medicine

University of Sydney NSW 2050, and ${ }^{4}$ CRC for Asthma and Airways NSW

Introduction The extent of emphysema could increase ventilation heterogeneity independently of its effects on airway narrowing. The aim of this study was to examine the relationship between emphysema extent on computed tomography scans (CT), and airway narrowing and ventilation distribution in COPD.

Methods 27 subjects with COPD underwent CT scanning, spirometry, DLCO and nitrogen washout by single and multiple breath techniques. Closing capacity $(\mathrm{CC} / \mathrm{TLC} \%)$, slope of phase III $\left(\Delta\right.$ phase $\left._{\text {III }}\right)$ and indices of ventilation distribution conductive (Scond) and diffusion-dependent airways (Sacin) were derived from washouts. Helical CT scans were performed at TLC. Emphysema extent was measured as low attenuation areas $<-910 \mathrm{HU}$ using Osirix program, expressed as \% of CT total lung volume.

Results Subjects were of mean (range) age 70 years (55-85), BMI 27.3 (17.5-42.7), $\mathrm{FEV}_{1}$ of 57 (34-92\%) \%predicted and DLCO of 50 (29-78) \%predicted. Emphysema extent was $22.7 \%(0.1-54.1)$. Geometric mean $(\mathrm{Cl})$ $\Delta$ phase $_{\| I}$ was 8.1 (6.8-9.6), Sacin was increased at $0.553 \mathrm{~L}^{-1}(0.477-0.641)$ and CC/TLC\% was $46 \%$ (44-48). Emphysema extent correlated with $\mathrm{FEV}_{1} /$ FVC $(r=-0.56, p=0.002)$, DLCO $(r=-0.63, p<0.001)$, BMI $(r=0.49, p=$ $0.009), \Delta$ phase $_{\| \mid}(r=0.46, p=0.017)$, and Sacin $(r=0.56 p=0.003)$. In multiple regression analysis, emphysema extent was predicted by $F E V_{1} / F V C$ and $\Delta$ phase $_{\text {III }}$ (model $\left.\mathrm{r}^{2}=0.46, \mathrm{p}=0.0005\right)$.

Conclusions The extent of emphysema increases the heterogeneity of ventilation independently of any effects on overall airway narrowing.

Supported by Australian Lung Foundation Webster Memorial Award, CRCAA.

Conflict of Interest No.
TP-059

RETINAL PHOTOGRAPHY AS A NON-INVASIVE MEASURE OF MICROVASCULAR DISEASE IN PATIENTS WITH COPD

S CHEW, AF HUTCHINSON, A WONG, CF MACDONALD, JA SAVIGE Department of Medicine, University of Melbourne (Northern), Vic, Australia

Introduction Early detection of patients with mild to moderate COPD at increased risk of cardiac events may improve management and reduce mortality as well as decrease health-care costs. However, no currently available biomarkers effectively predict individuals at cardiac risk. Retinal photography allows non-invasive visualization of the microvasculature and may represent a surrogate for demonstrating cumulative damage to the cardiac vasculature.

Aim This study describes the prevalence of retinal changes in COPD patients and examines whether retinal microvascular changes discriminate between COPD patients with and without known cardiac disease.

Methods One hundred fifty-one patients with COPD and 151 age- and gender-matched controls were studied. Patients completed a questionnaire and underwent retinal photography with a non-mydriatic retinal camera. Clinical details noted included the presence of traditional risk factors, known cardiac disease and current medications. Retinal photographs were coded and examined by two independent observers for abnormalities and microvascular changes. The association between previous cardiac disease and microvascular changes in cases and controls was examined using univariate and multivariate logistic regression models.

Results Patients with COPD had more severe microvascular changes compared with controls (OR 2.95, 95\% Cl 1.42-6.16, $\mathrm{p}=0.004$ ). Retinal microvascular changes were associated with current cardiac disease in patients with COPD (OR 8.30, 95\% Cl 3.60-19.11, $\mathrm{p}=0.000$ ) after controlling for traditional risk factors.

Conclusion Retinal photographs may represent a screening method for microvascular risk in patients with COPD.

Conflict of Interest No.

Journal Compilation (C) 2011 Asian Pacific Society of Respirology 
INJECTABLE VACCINES FOR PREVENTING PNEUMOCOCCAL INFECTION IN PATIENTS WITH COPD - A SYSTEMATIC REVIEW

\author{
JAE WALTERS ${ }^{1}$, S SMITH $^{2}$, P POOLE ${ }^{3}$, RH GRANGER ${ }^{1}$, \\ R WOOD-BAKER ${ }^{1}$ \\ ${ }^{1}$ Menzies Research Institute, University of Tasmania, Hobart, ${ }^{2}$ University of \\ Tasmania, Hobart 7001, and ${ }^{3}$ University of Auckland, Auckland, \\ New Zealand
}

Aim Pneumococcal vaccination is recommended for at-risk populations, including COPD, to prevent pneumococcal disease and complications. Recommendations in COPD guidelines are based on observational studies or trials not confined to COPD. We evaluated the efficacy of injectable pneumococcal vaccines in COPD.

Methods Databases were searched in a Cochrane review update for randomized trials of injectable polyvalent pneumococcal vaccines in adults with COPD. Two reviewers performed data extraction, data entry and study quality assessment.

Results Seven studies that randomized 1709 previously unvaccinated, immuno-competent participants with COPD to treatment or control were included. Five studies used 23-valent and 2 used 14-valent vaccines; 4 studies used a placebo control and 3 had adequate allocation concealment. Mean ages of participants ranged from 58 to 78 years. Primary outcomes: neither the reduction in likelihood of developing pneumonia, based on clinical or radiological diagnosis, from 6 studies $(n=1372)$ (OR $0.72,95 \% \mathrm{Cl} 0.51$ to 1.01), nor the reduction in likelihood of an acute exacerbation of COPD from 2 studies $(n=216$ ) (OR $0.58,95 \% \mathrm{Cl} 0.30$ to 1.13 ) achieved statistical significance. Secondary outcomes: there were no statistically significant effects of vaccination on reduction in hospital admissions or emergency department visits (1 study). There was no reduction in all-cause mortality (OR 0.94; 95\% $\mathrm{Cl} 0.67$ to 1.33 ) or deaths from cardio-respiratory causes (OR $1.07 ; 95 \% \mathrm{Cl}$ 0.69 to 1.66 ) for periods up to 48 months post-vaccination.

Conclusions Some benefit for injectable pneumococcal vaccines in COPD is possible but no statistically significant effects were found in this review, possibly due to inadequate numbers. Further randomized trials of adequate size are needed to confirm vaccine effectiveness in COPD, as suggested by observational studies.

Supported by Australian Government Dept of Health and Ageing.

Conflict of Interest No.

\section{IMPLEMENTATION OF COPD GUIDELINES IS LOW FOR PULMONARY REHABILITATION AND EXACERBATION PLANS: PILOT DATA}

\section{K JOHNSTON ${ }^{1}$, K GRIMMER-SOMERS ${ }^{1}$, M YOUNG ${ }^{2}$, R ANTIC ${ }^{3}$}

${ }^{1}$ International Centre for Allied Health Evidence, University of SA, SA, Australia, ${ }^{2}$ Transitional and Community Service, Royal Adelaide Hospital, $S A$, Australia, and ${ }^{3}$ Department of Thoracic Medicine, Royal Adelaide Hospital, SA, Australia.

Aim To examine the implementation of, and barriers and enablers to, six high-evidence recommendations for COPD management, in COPD hospital inpatients.

Method Observational, mixed methods study in consecutive COPD patients admitted to a tertiary hospital. Demographic, disease and admission characteristics are recorded. Implementation (or not) of smoking cessation, pulmonary rehabilitation, long-term oxygen use if hypoxaemic, medication use, vaccinations and plans for future exacerbations are determined from medical records and patient interviews. Interviews with medical officers examine their perspectives on recommendation implementation.

Results Of pilot data in 10 COPD patients (mean (SD) age 76(10) years, length of stay $7(6)$ days), 2 were current smokers and 4 had severe COPD (6 moderate). Highest levels of implementation were flu vaccination (completed by GPs, $n=10$ ), medication (but not spacer) use, and oxygen use if hypoxaemic (investigated and implemented in all suitable, $n=4$ ). Pulmonary rehabilitation was discussed with half of the patients, but only 2 severe patients with long length of stay accepted further rehabilitation. Exacerbation plans were in place for 1 patient, and newly initiated in 2 patients. Doctor interviews $(n=8)$ confirmed pulmonary rehabilitation was considered mostly for severely unwell patients, and use of exacerbation plans was inconsistent.

Conclusion Pilot data suggest pulmonary rehabilitation is offered and accepted by a small subset of COPD patients. Findings from this pilot will inform planned larger observational studies, and in turn, experimental studies to improve COPD care.

Supported by International Centre for Allied Health Evidence Seeding Grant.

Conflict of Interest No.

\section{PREDICTED 20-MINUTE WALK GOAL IS INACCURATE IN PATIENTS WITH CHRONIC OBSTRUCTIVE PULMONARY DISEASE (COPD)}

M ROBERTS ${ }^{1}$, T ROBINSON ${ }^{1,2}$

${ }^{1}$ Respiratory Ambulatory Care Service, and ${ }^{2}$ Ludwig Engel Centre for Respiratory Research, Westmead Hospital, NSW

\section{AN EVALUATION OF THE ROLE AND POTENTIAL BENEFITS OF THE CLINICAL PHARMACISTS INVOLVEMENT IN RESPIRATORY OUTPATIENT CLINIC}

T TSE ${ }^{1}$, T ALTHUNIAN ${ }^{2}$, J BURROWS ${ }^{2}$, I YANG ${ }^{1}$, P MASEL ${ }^{1}$

${ }^{1}$ Thoracic Department, The Prince Charles Hospital, Qld, and ${ }^{2}$ Pharmacy

Department, The University of Queensland, QId

Aim To evaluate the role and potential benefits of clinical pharmacists' involvement in respiratory outpatient clinic.

Method This study assessed the clinical significance of pharmacist interventions made in the respiratory outpatient clinics. The assessment was made by a multidisciplinary independent panel and by patients' respiratory physicians. The impact of pharmacist consultation services on medication compliance and patient satisfaction was evaluated. Finally, the number of patients with issues around medication knowledge was determined and clinical pharmacist cost was calculated.

Results A total number of 30 interventions were made among 28 patients. High- and extreme high-risk interventions were found by panel $(0-3.33 \%$ extreme and $3.57-13.33 \%$ high-risk interventions) and patients' respiratory physicians ( $3 \%$ extreme and $27 \%$ high-risk interventions). Additionally, clinical pharmacist involvement was associated with many benefits such as: improvement in medication compliance, high level of patient satisfaction and identification of patients with issues in medication knowledge.

Conclusion Clinical pharmacist interventions were estimated to prevent extreme and high risks that might happen due to drug-related problems. Clinical pharmacy consultation was associated with positive impact on other important measured outcomes.

Conflict of Interest No.

Journal Compilation (C) 2011 Asian Pacific Society of Respirology
Aerobic exercise training in the form of supervised 20-minute walks (20MW) reduces exertional dyspnoea in patients with COPD. 20MW goal (20MWG) distances, aiming for a training effect, are generated from a baseline submaximal test (6-minute walk (6MWD), where $20 \mathrm{WG}=0.8 \times 6 \mathrm{MWD} / 6 \times 20$.

Aim To compare 20MWG with actual initial 20MW achieved and to examine the predictors of 20MWG achievers (GA).

Methods Retrospective review of 404 patients, $58 \%$ male, age $68 \pm 9$ years (mean \pm SD), FEV $12 \pm 17 \%$ predicted, who completed pulmonary rehabilitation (PR). Patients were assessed at baseline and post-completion of PR. Initial 20MWG was calculated from the best of two 6MWD at initial assessment and GA were defined as patients who achieved their 20MWG at their first visit to PR.

Results For the group, there was a statistically significant but not clinically significant difference between 20MWG and actual 20MW achieved (1025 \pm $320 \mathrm{~m}$ vs. $1086 \pm 398 \mathrm{~m}, \mathrm{p}<0.05$, paired $t$-test). The 264 patients $(65 \%)$ who achieved their 20MWG exceeded the goal by $182 \pm 141 \mathrm{~m}$, whereas the 140 patients who did not achieve their 20MWG fell short by $173 \pm 168 \mathrm{~m}$. There was no significant difference between GA and non-GA in age or lung function, but GA had a higher initial GMWD, with fewer rests, lower dyspnoea score and lower HR at start and finish ( $\mathrm{p}<0.05$, unpaired $t$-test). GA were also more likely to have a clinically significant response to PR, measured by 6MWD, compared with non-GA (mean change $63 \mathrm{~m}$ vs. $37 \mathrm{~m}, \mathrm{p}<0.05$, chi-square). Conclusion 20MW goals as currently calculated either significantly underestimate or overestimate actual 20MW achieved. It may be that in non-GA, the 6MWD is functioning as a true maximal test and these are a group of patients who are truly ventilatory-limited, rather than deconditioned.

Conflict of Interest Nil. 
COPD SIG: POSTER SESSION 2

TP-064

RAGE POLYMORPHISMS DO NOT CONTRIBUTE TO CHRONIC OBSTRUCTIVE PULMONARY DISEASE

SAI RUPA BASKAR, B SHELTON, S BALTIC, C MITRPANT, PJ THOMPSON

Lung Institute of Western Australia, University of Western Australia, Perth, WA

The receptor for advanced glycation end products (RAGE) is a key candidate for promoting a self-perpetuating cycle of inflammation and thereby is a major contributor to numerous chronic disease states. The potential of RAGE to function as a switch converting a transient inflammatory response such as one generated by cigarette smoke to sustained cellular dysfunction allows it to act as a mediator for ongoing inflammation in chronic obstructive pulmonary disease (COPD). Although the molecular mechanisms regulating RAGE expression have not been fully elucidated, altered RAGE activity arises from polymorphisms within the RAGE gene and its promoter. Three polymorphisms in the RAGE promoter $(-374 \mathrm{~T} / \mathrm{A},-429 \mathrm{~T} / \mathrm{C}$ and a $63 \mathrm{bp}$ deletion from -407 to -345 ) increase transcriptional activity and RAGE expression. The RAGE G82S allele results in an increased ligand-binding affinity and activation of the inflammatory mediators with subsequent up-regulation of inflammatory response. The aim of this pilot cross-sectional study was to investigate the relationship between three known RAGE polymorphisms (-374T/A, 63 bp deletion, G82S) and COPD and disease severity.

Methods Genomic DNA was isolated from peripheral blood lymphocytes. PCR and TaqMan assays were used to genotype the three RAGE polymorphisms in 67 COPD patients, 70 healthy non-smokers and 70 healthy smokers. $\mathrm{FEV}_{1}$ was measured in all subjects. Disease severity was defined using GOLD guidelines.

Results There was no statistically significant association between $63 \mathrm{bp}$ deletion and COPD $(p=0.61),-374 \mathrm{~T}>\mathrm{A}$ and COPD $(p=0.58)$, G82S and COPD $(p=0.42)$.

Conclusion No association was found between the $-374 \mathrm{~T}>\mathrm{A}, 63$ bp deletion and G82S polymorphisms and COPD, disease severity or FEV ${ }_{1}$ and $F E V_{1} /$ FVC. Future studies with greater sample sizes are required.

Nomination Nil.

Conflict of Interest No.

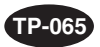

\section{EFFECT OF CIGARETTE SMOKE EXTRACT ON NEUTROPHILS} ISOLATED FROM HUMAN PERIPHERAL BLOOD

H ZHONG ${ }^{1}$, M TOOZE $^{1}$, LG WOOD ${ }^{1,2}$, JL SIMPSON ${ }^{1,2}$, PAB WARK $^{1,2}$

${ }^{1}$ Centre for Asthma and Respiratory Disease, Hunter Medical Research

Institute, University of Newcastle, NSW, and ${ }^{2}$ Department of Respiratory and Sleep Medicine, John Hunter Hospital, NSW

Aims Increased numbers of neutrophils are found in the lungs of COPD patients, which contribute to airway inflammation. While cigarette smoke exposure is the major risk factor for COPD, it is unclear how cigarette smoke modifies neutrophil function and activity. This study aimed to assess the effect of cigarette smoke extract (CSE) on neutrophils in an in vitro model.

Methods Neutrophils were isolated from peripheral blood donated by volunteers using Percoll density gradient centrifugation. Neutrophils were seeded in 24 well plates $\left(10^{6}\right.$ cells/well), exposed to different concentrations of CSE $(10 \%, 1 \%)$ and monitored at 2,4 and 18 hours. At each time point, viability of neutrophils was measured by trypan blue exclusion and supernatant was collected for measurement of CXCL8 release by ELISA (R\&D Systems). Results

\begin{tabular}{llll}
\hline Neutrophil Viability (\%) & \multicolumn{2}{c}{ CXCL8 Concentration $(\mathrm{pg} / \mathrm{mL})$} \\
\hline Groups & Time point & \\
& 2 hours & 4 hours & 18 hours \\
$10 \%$ CSE & $94.4 \pm 11$ & $90.3 \pm 3.0$ & $79.9 \pm 1.5$ \\
$1 \%$ CSE & $95.1 \pm 1.5$ & $91.0 \pm 2.0$ & $89.6 \pm 1.8$ \\
Media & $100 \pm 0$ & $97.3 \pm 1.6$ & $94.6 \pm 1.2$ \\
Groups & Time point & & \\
& 2 hours & 4 hours & 18 hours \\
$10 \%$ CSE & 9.409 & 32.929 & 446.583 \\
$1 \%$ CSE & 8.915 & 18.841 & 65.195 \\
Media & 6.164 & 6.47 & 59.9 \\
\hline
\end{tabular}

Conclusions In neutrophils exposed to CSE, viability is maintained and CXCL8 release increases with increasing dose of CSE. We conclude that cigarette smoke stimulates an inflammatory response by neutrophils, which would contribute to the inflammatory burden in the airways in COPD.

Supported by Chinese Scholarship Council.

Conflict of Interest No.
TP-066

\section{SOLUBLE RAGE IS DEFICIENT IN NEUTROPHILIC ASTHMA AND COPD}

M SUKKAR ${ }^{1}$, LG WOOD ${ }^{2}$, M TOOZE ${ }^{2}$, J SIMPSON², VM MCDONALD ${ }^{2,3}$, P GIBSON 2,3, P AB WARK ${ }^{2,3}$

${ }^{1}$ Respiratory Research Group, Faculty of Pharmacy, The University of Sydney, ${ }^{2}$ Centre for Asthma and Respiratory Disease, Hunter Medical Research Institute, University of Newcastle, and ${ }^{3}$ Department of Respiratory and Sleep Medicine John Hunter Hospital, Newcastle

Introduction The receptor for advanced glycation end products (RAGE) mediates neutrophil trafficking and is implicated in the pathogenesis of chronic airways disease. We determined whether changes in airway and systemic levels of soluble RAGE (which acts as a receptor decoy to limit RAGE activation) and RAGE ligands are related to neutrophilic inflammation in asthma and COPD.

Methods Bronchial lavage (BL) fluid from subjects with moderate-severe persistent asthma or COPD, and healthy controls were analysed for neutrophils, total sRAGE (cleaved and secreted), secreted sRAGE (esRAGE) and the RAGE ligands HMGB1 and serum amyloid A (SAA). Systemic levels SRAGE and esRAGE were also determined in asthmatic and COPD subjects.

Results Subjects with neutrophilic asthma or COPD had undetectable levels of BL SRAGE, while levels of SRAGE in asthma or COPD without neutrophilia were similar to controls (433.4 (866.2); 211.1 (293.6); 445.3 (451.3) pg/mL (sd), respectively). Systemic levels of SRAGE in asthmatic and COPD subjects with sputum neutrophilia (409.5 (215.2) and 285.5 (216.3) pg/mL (sd), respectively) had significantly lower levels of sRAGE compared to those without neutrophilia (462.4 (165.9) and 496.9 (274) pg/mL (sd), respectively). There was a strong positive correlation between esRAGE and total SRAGE in both the airways and systemically suggesting that the dominant source of RAGE is the secreted isoform. BL levels of HMGB1 were similar in all subject groups, while SAA was below detectable levels.

Conclusion We have shown that deficiency in sRAGE in asthma and COPD is selectively associated with the presence of neutrophilic airway inflammation. Neutrophilic airways disease might be amenable to treatment by alleviating deficiencies in soluble RAGE.

Conflict of Interest No.
COMPARING FACTOR VIII WITH COLLAGEN IV FOR IMMUNOSTAINING OF VESSELS

A SOLTANI, D REID, SS SOHAL, S WESTON, HK MULLER, $R$ WOOD-BAKER, EH WALTERS

Menzies Research Institute, University of Tasmania, Hobart, TAS

Introduction Factor VIII (F8) and collagen IV (C4) antibodies are used for quantifying vessels in tissue sections. We compared these two antibodies for vessels staining in bronchial biopsies (BB) in COPD.

Methods BB from 7 healthy non-smokers $(\mathrm{H}-\mathrm{N})$ and 28 COPD subjects were stained for both antibodies. Number, area and mean vascular size (MVS) (surface area/vessel number) of vessels in the lamina propria (LP) to the depth of $150 \mu \mathrm{m}$ were measured and compared between the two antibodies and are reported as median (range).

Results Number of vessels was not significantly different between the two methods of staining. In COPD and H-N, vascular area $\left(\mu \mathrm{m}^{2} / \mu \mathrm{m}^{2}\right.$ of $\left.\mathrm{LP} \times 100\right)$ stained with F8 was less than that with C4 $(4.1(1.8-8)$ vs. $6(2-10.3), p<0.01$ and $3.9(2.6-8.7)$ vs. $6.8(3.7-8.9), p<0.05)$. MVS with F8 was less than that with $\mathrm{C} 4$ in COPD and H-N (112 (60-316) vs. $204(110-457), \mathrm{p}<0.01$ and 70 $(45-198)$ vs. $183(67-286), p<0.05)$. The COPD group had higher MVS compared to $\mathrm{H}-\mathrm{N}$ with F8 staining $(112(60-316)$ vs. $70(45-198), \mathrm{p}<0.05)$ but not C4.

Conclusions $\mathrm{F} 8$ and $\mathrm{C} 4$ probably stain somewhat different structures in $\mathrm{BB}$ sections from both control and COPD subjects; F8 stains smaller and probably younger vessels. Higher MVS with F8 but not C4 in the LP of COPD group compared to H-N suggests predominance of older vessels in this compartment. Further investigation of angiogenic activity in COPD is needed.

Supported by NHMRC.

Conflict of Interest NIL. 


\section{C-REACTIVE PROTEIN (CRP) LEVELS FLUCTUATE WIDELY IN STABLE COPD: IMPLICATIONS FOR DEFINING AND PREDICTING EXACERBATIONS}

MA THOMPSON ${ }^{1,2}$, GP ANDERSON ${ }^{2}$, AF HUTCHINSON $^{1}$, LB IRVING ${ }^{1}$ ${ }^{1}$ Department of Respiratory \& Sleep Medicine, Royal Melbourne Hospital, Parkville, Vic., and ${ }^{2}$ University of Melbourne Dept. Medicine \& Pharmacology, Parkville, Vic.

Introduction Previous studies have shown that C-reactive protein levels increase at the onset of some COPD exacerbations; however, there is limited data on the normal fluctuation in CRP levels in stable patients.

Aim To investigate within patient variation in CRP levels to determine the magnitude of normal day-to-day fluctuations in stable patients and the correlation with patients' perception of symptom severity.

Methods Early morning CRP levels were measured on days 1, 35 and 6 from 22 patients from the Melbourne COPD Cohort (GOLD Category II-IV) who identified themselves as stable. Patients recorded daily symptom scores including: Borg dyspnoea scale at rest, severity of wheeze, cough, dyspnoea, change in sputum colour or volume, night-time waking and the presence of viral symptoms. CRP levels were measured by the clinical pathology service and using a point-of care device. Variation in CRP levels in stable COPD and correlation between change in CRP levels and symptoms were analysed. Results Sixteen of the 22 patients were stable for 7 days and 6 experienced an exacerbation during the study observation period. Mean CRP level on day 1 was $8 \mathrm{mg} / \mathrm{L}$ range 0.6 to $32 \mathrm{mg} / \mathrm{L}$. Mean within patient variation in CRP over 1 week was $3.03 \mathrm{mg} / \mathrm{L}$ range 0.27 to $22 \mathrm{mg} / \mathrm{L}$ in stable patients and in AECOPD patients $4.62 \mathrm{mg} / \mathrm{L}$, range 1.8 to $17.8 \mathrm{mg} / \mathrm{L}$. A marked increase in CRP levels correlated with increased symptoms in $80 \%$ of patients.

Conclusion Fluctuations in CRP levels are non-specific for the onset of an AECOPD with considerable variations in levels when patients are stable. Conflict of Interest No.

\section{VALUE OF DAILY SYMPTOM MONITORING FOR PREDICTION OF SEVERE EXACERBATIONS OF COPD}

\author{
JAE WALTERS ${ }^{1}, K_{\text {WILLS }}^{1}$, L BLIZZARD ${ }^{1}$, A ROBINSON ${ }^{1}$, P TURNER $^{2}$, \\ R WOOD-BAKER ${ }^{1}$, EH WALTERS \\ ${ }^{1}$ Menzies Research Institute, and ${ }^{2}$ University of Tasmania; Hobart 7001 , \\ Australia
}

Aim Patient-completed diaries monitoring changes in key symptoms in COPD are often used to recognize acute exacerbations (AE) both to prompt additional treatment and monitor treatment efficacy. We assessed diary compliance and the predictive value of major symptoms of AEs which required hospital attendance.

Methods Inpatients recruited during an AE of COPD completed daily paper or web-based diaries for 12 months, recording changes from their stable state for: breathlessness, cough, sputum, subjective 'wellness', physical activity and use of reliever (7-point scale, mid-pt $=$ no change). The predictive value of current and lagged symptom scores was compared for each and between symptoms. Diagnostic accuracy was assessed by area under the curve (AUC) and at specific cut-points.

Results In 55 participants (18M, 37F) with mean age $67 \pm 10$ and mean $\mathrm{FEV}_{1} \%$ predicted $35 \pm 14$, there were 116 such $\mathrm{AEs}$ involving 32 patients. Duration of diary keeping was shorter with lower education attainment $(p=$ 0.05 ), but compliance did not vary for other demographic or clinical factors. Daily compliance while diaries were being kept was $85 \%$. Excluding the current day, the best predictor was the distributed lag score over 6 days, sputum changes giving the strongest signal; relative risk $1.65(95 \% \mathrm{Cl} 1.21$ to 2.23$)$ with most of the signal in the 2 days prior to the AE. Little was gained by combining symptoms. The predictive value was moderate $\mathrm{AUC}=0.67$.

Conclusions Compliance with symptom diaries in severe COPD is surprisingly good. However, with only a weak signal for an impending AE requiring hospital attendance up to 48 hours before and for lagged symptom scores over 6 days before, with low positive predictive values, the utility of keeping daily symptom diaries for raising alerts for impending severe AEs in COPD is questionable.

Supported by DHHS Tasmania \& Commonwealth DOHA.

Conflict of Interest No.

\section{TP-069}

OPTIMUM DURATION OF CORTICOSTEROID THERAPY FOR ACUTE EXACERBATIONS OF CHRONIC OBSTRUCTIVE PULMONARY DISEASE

\section{JAE WALTERS ${ }^{1}$, W WANG ${ }^{2}$, C MORLEY ${ }^{2}$, A SOLTANI $^{1}$, R WOOD-BAKER $^{1}$} ${ }^{1}$ Menzies Research Institute, and ${ }^{2}$ University of Tasmania; Hobart, Australia

Aim Current guidelines recommend systemic corticosteroid treatment for acute exacerbations (AE) of COPD to reduce treatment failure and shorten recovery; however, they do not define the optimum duration of treatment. Intermittent corticosteroid use is cumulatively associated with adverse effects; thus, shorter treatment could reduce risks. We compared short ( $\leq 7$ days) versus longer ( $>7$ days) systemic corticosteroid (SCS) treatment for $\mathrm{AE}$ of COPD in a systematic review.

Methods We searched databases for randomized controlled trials of SCS for AE COPD. Two reviewers performed data extraction and assessed study quality.

Results Seven studies with 288 inpatient participants were identified; 3 published as abstracts for which data were not available did not contribute to meta-analyses. No study specified diagnostic criteria for COPD and only one specified AE criteria. Short course treatment varied between 3-7 days and longer duration 10-15 days; 5 studies used oral prednisolone (dose $30 \mathrm{mg}, 4$ studies, 1 tapered dose) and 2 studies used intravenous SCS treatment. Mean ages of participants ranged from 64 to 73 years. Primary outcomes: likelihood of treatment failure did not differ by duration of treatment (odds ratio $0.82 ; 95 \%$ $\mathrm{Cl} 0.24$ to 2.79) (3 studies, $\mathrm{n}=146$ ). $F E V_{1}$ did not differ significantly when measured up to 7 days (Mean difference (MD) $-0.07 \mathrm{~L} ; 95 \% \mathrm{Cl}-0.19$ to 0.05 ) or after 7 days (MD $-0.02 \mathrm{~L} ; 95 \% \mathrm{Cl}-0.10$ to 0.06 ) (4 studies, $n=197$ ). Secondary outcomes: limited data ( 1 study) precluded meta-analysis for readmission or mortality. The likelihood of an adverse event (4 studies, $n=102)$ was not significantly lower for shorter SCS (OR $0.58 ; 95 \% \mathrm{Cl} 0.14$ to 2.40 ).

Conclusions We found no significant differences between short ( $\leq 7$ days) and longer ( $>7$ days) corticosteroid therapy for AE of COPD. This has implications for clinical practice and may reduce adverse effects for patients, shorten hospital admissions and reduce costs, but more studies are needed to confirm these findings.

Supported by Australian Government Dept of Health and Ageing.

Conflict of Interest No.

Journal Compilation (C) 2011 Asian Pacific Society of Respirology

\section{EXPLORING FACTORS THAT INFLUENCE THE SELF- MANAGEMENT OF EXACERBAITONS IN PATIENTS WITH CHRONIC OBSTRUCTIVE PULMONARY DISEASE \\ T TSE ${ }^{1}$, Z HASSAN², I YANG ${ }^{1}$, J BURROWS ${ }^{2}$, J SERGINSON', $L_{\text {MCCATHY }}$ \\ ${ }^{1}$ Thoracic Department, The Prince Charles Hospital, Qld, and ${ }^{2}$ Pharmacy Department, The University of Queensland, Qld}

Aim To explore factors which influence the self-management of exacerbations in patients with COPD.

Methods A pilot cross-sectional study was undertaken to assess patients' compliance with their action plan and their action taken prior to an admission. Patients were interviewed during an admission to hospital for exacerbation of COPD. The effect of pulmonary rehabilitation on patients' knowledge of COPD was also assessed.

Results $70 \%$ of patients were provided with a written action plan, and $25 \%$ with a verbal action plan. In response to an exacerbation, more than $70 \%$ of the patients stated that they used their action plan. However, where action plans were not adequately utilized, patients delayed seeking medical attention and failed to initiate oral prednisolone and antibiotics during an exacerbation despite being prescribed an emergency supply of these medications. Pulmonary rehabilitation had a positive outcome towards enhancing the patients' knowledge of COPD. Clinical pharmacists have limited involvement in terms of COPD and smoking cessation education.

Conclusion The need to offer a thorough self-management program along with providing a more comprehensive written action plan will encourage patients to start early treatment and follow their action plans. Encouraging collaboration between the HCP and patients encourages self-management through discussing and agreeing on goals of treatment and developing a personalized written action plan.

Conflict of Interest No. 

A SYSTEMATIC REVIEW

TA SMITH $^{1}$, L LAM $^{2}$, PM DAVIDSON ${ }^{3}$, CR JENKINS $^{4}$, J INGHAM ${ }^{1}$

${ }^{1}$ Cunningham Centre for Palliative Care, Sydney NSW, ${ }^{2}$ Notre Dame University, Sydney NSW, ${ }^{3}$ Curtin University, Sydney NSW, and ${ }^{4}$ Woolcock Institute, Sydney NSW

Context Dyspnoea is a common symptom in COPD and increases during exacerbations. When respiratory failure supervenes, and assisted ventilation is required, non-invasive ventilation (NIV) is the treatment of choice.

Objective To determine if NIV relieves dyspnoea in inpatients with acute respiratory failure due to exacerbations of COPD.

Data Sources English language randomized controlled trials (RCTs) published prior to August 2010 were identified using Medline, Embase, CINAHL, PsychINFO and PubMed. Additional studies were identified by reviewing the reference list of included studies. Search terms included NIV, NIPPV, NPPV, Bilevel CPAP, BiPAP, artificial ventilation, COPD and randomized controlled trial.

Study selection RCTs comparing usual medical care (UMC) to UMC plus NIV and measuring dyspnoea at relevant time points were included. Abstracts for potentially relevant articles were extracted by one author. These were assessed by a second author to ensure inclusion criteria were met. Articles were reviewed to determine if dyspnoea was measured and appropriate statistical analysis reported.

Results The search yielded 327 individual articles. Four articles met the review criteria. Three articles find that NIV relieved dyspnoea to a statistically significant level and two suggested that the relief of dyspnoea is clinically significant.

Discussion In spite of the common use of NIV to relieve dyspnoea, little work has analysed efficacy in terms of this patient-reported outcome. While our results may suggest NIV relieves dyspnoea, reporting or methodological flaws in several articles limit the strength of the conclusions that may be drawn. These limitations make the conclusion that NIV relieves dyspnoea contentious.

Conclusion Despite over two decades of studies investigating NIV, the therapeutic impact on breathlessness is poorly described. Understanding the impact of NIV on patient-reported outcomes is of critical importance in clinical care. Conflict of Interest None.

\section{TP-073}

ACUTE RECOVERY FROM COPD EXACERBATIONS MEASURED USING FOT

C DIBA ${ }^{1,2,3}$, S TIMMINS ${ }^{1,2,3,4}$, CM SALOME $E^{1,2,3}$, FQ WEN $^{1,5}$, N BEREND $^{1,2,3}$ GG KING ${ }^{1,2,3,4}$

${ }^{1}$ Woolcock Institute of Medical Research NSW 2050, ${ }^{2}$ CRC for Asthma NSW $2050,{ }^{3}$ The University of Sydney NSW 2050, ${ }^{4}$ Department of Respiratory Medicine and Department of Nuclear Medicine Royal North Shore Hospital NSW 2065, and ${ }^{5}$ West China Hospital, West China School of Medicine, Sichuan University, China

Spirometric measures are difficult to perform in COPD patients, especially during an exacerbation. The forced oscillation technique (FOT) is ideal for measuring lung function in COPD patients as measurements are made during tidal breathing, it is effort independent and measures both respiratory system resistance (Rrs) and reactance (Xrs). It has been suggested that FOT parameters may be more sensitive at tracking with recovery from a COPD exacerbation than spirometry.

Aim To compare the rate of recovery from a COPD exacerbation measured by FOT and spirometry.

Methods 28 COPD subjects hospitalized for a COPD exacerbation had daily spirometry and FOT $(6 \mathrm{~Hz})$ measurements made for the duration of their hospital stay. Rate of change in FOT and spirometric parameters was calculated for individual patients as the slope of the regression between length of stay (days) and Rrs, $\mathrm{Xrs}_{\mathrm{r}} \mathrm{FEV}_{1}$ and FVC, expressed as a percentage of admission values.

Results Mean $\pm 95 \% \mathrm{Cl}$, age was $61.0 \pm 3.0$ years and $\mathrm{FEV}_{1} / \mathrm{FVC}$ was 0.45 \pm 0.04 . There was no significant difference in mean \pm SD slope between $\mathrm{FEV}_{1}$ (2.20 \pm 5.87$), F V C(0.69 \pm 4.79)$, Rrs $(-0.89 \pm 12.31)$ and Xrs $(-2.10 \pm 12.31)$ $(p=0.24)$. There was no significant difference between admission and discharge values for FEV $(0.81 \pm 0.40$ vs. $0.82 \pm 0.37 \mathrm{~L}, \mathrm{p}=0.07)$, FVC $(1.77 \pm$ 0.55 vs. $1.76 \pm 0.51 \mathrm{~L}, \mathrm{p}=0.46)$, Rrs $\left(6.28 \pm 2.94\right.$ vs. $5.77 \pm 2.43 \mathrm{cmH}_{2} \mathrm{O} /(\mathrm{L} /$ sec), $\mathrm{p}=0.16)$ and $\mathrm{Xrs}\left(-4.31 \pm 2.82\right.$ vs. $\left.-3.83 \pm 2.00 \mathrm{cmH}_{2} \mathrm{O} /(\mathrm{L} / \mathrm{sec}), \mathrm{p}=0.14\right)$. Conclusion Change in lung function with exacerbation resolution was highly variable among patients. However, as a group, there was no significant difference in the rate of recovery from a COPD exacerbation measured by FOT and spirometry.

Support CRC for Asthma and Airways.

Conflict of Interest No.

\section{CYSTIC FIBROSIS SIG: POSTER SESSION}

TP-074

WHERE DOES IT GO? DYNAMICALLY TRACKING THE FATE OF FLUID FROM LUNG INSTILLATIONS IN MICE

M DONNELLEY ${ }^{1,2,3}, \mathrm{~K} \mathrm{SIU}^{4,5}$, A JAMISON $^{6}$, D PARSONS ${ }^{1,2,3,7}$

${ }^{1}$ Respiratory \& Sleep Medicine, Women's \& Children's Hospital, ${ }^{2}$ Paediatrics \& Reproductive Health and ${ }^{3}$ Centre for Stem Cell Research, University of Adelaide; ${ }^{4}$ School of Physics, ${ }^{5}$ Centre for Synchrotron Science and ${ }^{6}$ Division of Biological Engineering, Monash University, Melbourne, and ${ }^{7}$ Women's \& Children's Health Research Institute

Introduction In mice, the most direct lung dosing method delivers the agents directly into the trachea. For our cystic fibrosis gene-therapy studies, we deliver fluids - an airway pretreatment followed by a lentiviral vector - directly into the mouse trachea to target conducting airways. Despite using standardized delivery techniques, we see substantial variability in the amount and location of gene transfer.

Aim The aim of this experiment was to use synchrotron X-ray imaging to track the dynamics of fluid doses delivered into the live mouse trachea.

Methods Four nembutal anaesthetized C57BI/6 mice were imaged on the BL20B2 beamline at the SPring-8 synchrotron. Mice were intubated and ventilated at $80 \mathrm{br} / \mathrm{min}$ with 1 image captured per breath. After 1 minute of baseline, a $15-\mu \mathrm{L}$ sample of iodine-based contrast fluid (a surrogate for our airway pretreatment or gene-vector) was delivered over 30 seconds. Following 20 minutes of data collection, an additional $15 \mu \mathrm{L}$ bolus was delivered over 3.6 seconds. Image capture continued for a further 10 minutes. Frame differencing was used to reveal fluid motion.

Results Substantial dose losses may occur upon delivery into mouse trachea via immediate retrograde fluid motion. The speed of bolus delivery into lung may also influence the relative targeting of conducting airways and deep lung.

Conclusion Our findings suggest the need for, and permit, much greater attention to the specifics of the dosing setting - animal orientation, dose volume, dosing speed - and enable improvements in dosing technique design. Support NH\&MRC, Australian Synchrotron ISAP, http://www.cure4cf.org, JASRI.

Conflict of Interest Nil.
OPTIMAL DELIVERY OF CYSTIC FIBROSIS (CF) THERAPY FROM COMMONLY RECOMMENDED NEBULIZERS

LK LIEW ${ }^{1}$, CL GANGELLL ${ }^{2}$, EN SCHULTZ ${ }^{1}$, S MINOCCHIERI ${ }^{1}$, JT BURCHELL', SG DEVADASON ${ }^{1}$

${ }^{1}$ School of Paediatrics and Child Health, University of Western Australia, Perth, WA, and ${ }^{2}$ Division of Clinical Sciences, Telethon Institute for Child Health Research, Perth, WA

Introduction Use of efficient nebulizers can enhance the quality of life of CF patients by reducing the treatment time and improving drug delivery efficiency. The aim of this study was to determine which commonly recommended nebulizer was optimal for delivery of the most commonly used therapies to CF.

Methods Seventeen children with CF (8-17 years) were recruited. Delivery of three commonly used CF therapies ( $7 \%$ hypertonic saline $(4 \mathrm{~mL}, 0.07 \mathrm{~g} /$ $\mathrm{mL})$, Tobramycin $(4 \mathrm{~mL}, 20 \mathrm{mg} / \mathrm{mL}$ ) and Pulmozyme $(2.5 \mathrm{~mL}, 1 \mathrm{mg} / \mathrm{mL}))$ by two vibrating membrane nebulizers, the eFlow rapid and the Aeroneb Go, and a jet nebulizer LC Sprint Junior with PariBoy SX $(5.1 \mathrm{~L} / \mathrm{min})$ were tested. For each drug-nebulizer combination (in random order), each child was asked to inhale through an inspiratory filter, and drug delivery to the filter was measured. Pulmozyme was quantified using an enzymatic activity assay, tobramycin was measured using HPLC and hypertonic saline was measured using conductivity. Total nebulization time was recorded.

Results The results showed that there was no difference in the amount of drug delivered to patients when the 3 nebulizers were compared for all three therapies $(p>0.05)$. However, the nebulization time for the eFlow rapid was significantly shorter than that for the Aeroneb Go and LC Sprint Junior. Similarly, the nebulization time for Aeroneb Go was shorter than that for the LC Sprint Junior ( $p>0.01)$ for all therapies).

Conclusion Overall, there were no significant differences between nebulizers in delivered dose for three forms of CF therapy, due to inter-patient variability. Despite this, both vibrating membrane nebulizers had shorter nebulization times than the LC Sprint Junior, with the eFlow rapid delivering drug in the shortest time.

Conflict of Interest Nil.

Journal Compilation @ 2011 Asian Pacific Society of Respirology 


\section{SUPERIOR VENA CAVA SYNDROME DUE TO TOTAL IMPLANTABLE VENOUS ACCESS DEVICES IN CYSTIC FIBROSIS}

\author{
D SMITH ${ }^{1}$, D REID ${ }^{1}$, A TAl $^{1}$, P MASEL $^{1}$, R SLAUGHTER ${ }^{2}$, V MOORE $^{1}$, \\ $S$ BELL ${ }^{1}$ \\ Departments of ${ }^{1}$ Thoracic medicine and ${ }^{2}$ Radiology, The Prince Charles \\ Hospital, QId
}

Introduction As the life expectancy of patients with cystic fibrosis (CF) increases, treatment-related morbidity is increasingly recognized. Totally implantable venous access devices (TIVADs) offer reliable long-term central venous access but are associated with recognized complications including venous thrombosis. Superior vena cava syndrome (SVCS) however has been rarely reported in this setting. We report a single CF Centre's experience of SVCS associated with TIVADs.

Methods Retrospective review of episodes of SVCS in patients with CF and a TIVAD attending the adult CF Centre, Prince Charles Hospital, Queensland.

Results Between February 2008 and December 2009, five episodes of SVCS occurred in 60 patients with TIVADs from a clinic population of 251 patients. All of the affected patients were female, with moderately severe lung disease (Mean FEV 1 predicted $45.8 \%$ ). No patients had a recognized thrombophilia. Four TIVADs were inserted at a centre different to our own, three were on oestrogen-based contraception, and two suffered with dehydration at presentation. SVCS treatment consisted of anticoagulation (5), line removal (4), angioplasty (2), thrombolysis (1), SVC reconstruction at transplantation (1).

Conclusion TIVADS have a relatively low complication rate, but female gender, oestrogen-based contraception and hydration status appear to be significant risk factors for TIVAD-related thrombosis.

Conflict of Interest No.

\section{DIFFERENCES IN LONGEVITY OF LENTIVIRAL LUCIFERASE EXPRESSION IN AIRWAYS OF NORMAL AND CYSTIC FIBROSIS MICE}

P CMIELEWSKI ${ }^{1,2}$, D ANSON ${ }^{3}$, D PARSONS ${ }^{1,2,4,5}$

${ }^{1}$ Respiratory \& Sleep Medicine, ${ }^{2}$ Department of Paediatrics, ${ }^{4}$ Centre for Stem Cell Research, University of Adelaide, ${ }^{3}$ SA Pathology, Women's \& Children's Hospital, CYWHS, and ${ }^{5}$ Women's \& Children's Health Research Institute,

SA, Australia

Noninvasive bioluminescence imaging has allowed for rapid in vivo quantification of long-lasting gene transfer in experimental animals. We are testing the longevity of a single nasal delivery of our lentiviral (LV) gene transfer system in mouse airways.

Methods Normal (C57BI/6) and cystic fibrosis (CF) mice received a nasal bolus of lysophosphatidylcholine (LPC) or a control (PBS) pretreatment 1 hour prior to delivery of a LV vector containing the reporter-gene luciferase (LVLuc). Another control group received LPC 1 hour prior to an empty vector (LV-MT). Bioluminescence was measured at 1 week, 1, 3, 6, 9, 12, 15, 18, 21 and 24 months post-LV dosing to assess gene transfer.

Results Normal mice: Mice that received LPC/LV-Luc treatment had significantly greater gene transfer compared to the two control groups at all time points $(p<0.05, R M A N O V A)$. No luminescence was detected in mice treated with LPC/LV-MT. Unexpectedly, luciferase activity was also detected in the lung. There was no difference in lung luminescence between the LPC and PBS pretreated mice that received LV-Luc. CF mice: A statistically significant increase in nasal luminescence persisted for up to 6 months following LPC/ LV-Luc ( $p<0.05$, RM ANOVA). Similar to normal mice, there was no statistical difference in lung luminescence between mice that received LPC and PBS LV-Luc.

Conclusions Lentiviral luciferase gene expression was significantly improved in mouse nasal airways using LPC pretreatment in both strains. However, the longevity of transduction was reduced in CF mice, which may, in part, be due to reduced animal numbers at the later time points tested. Supported by NH\&MRC.

Conflict of Interest No.

\section{TP-077}

\section{THE EFFECTS OF NINTENDO-WII@ EXERCISE TRAINING IN} ADULTS WITH CYSTIC FIBROSIS

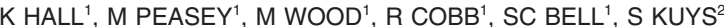

${ }^{1}$ Adult Cystic Fibrosis Centre, The Prince Charles Hospital, Brisbane, Queensland, and ${ }^{2}$ Allied Health Research Collaborative, Metro-North Health District, Queensland/Griffith Health Institute, Griffith University, Queensland

Background The Nintendo-Wiiß facilitates exercise-based programs that may be considered novel, fun and potentially motivating. Objective exercise outcomes using the Wii have yet to be reported in the cystic fibrosis (CF) adult population.

Aim To investigate Nintendo-Wiiß exercise training compared with standard exercise in adult CF patients whilst hospitalized for treatment of a pulmonary exacerbation.

Methods A within-subjects, randomized cross-over study. Adult CF participants received two 15-minute exercise treatment sessions within a 48-hour period, at least 1 day apart, during the last days of hospitalization. Wii exercise consisted interval training with games such as boxing, dancing and track exercises. Standard exercise consisted of interval training on treadmill or cycle ergometer at $60-80 \%$ of heart rate maximum.

Results 19 participants completed the study (mean (SD) age 27 (7) years, $47 \%$ females), with a mean $\mathrm{FEV}_{1} \%$ of $51(21) \%$. During exercise, no difference was found between groups in average heart rate $(p=0.98)$, oxygen desaturation $(p=0.46)$, Borg Rate of Perceived Exertion $(p=0.39)$ or Modified Borg for dyspnoea $(p=0.61)$. On VAS $(0-10)$, participants reported the Wii program to be more enjoyable $(p<0.01)$ and less fatiguing $(p=0.05)$. Participants rated both exercise sessions as equally effective $(p=0.25)$.

Conclusions This study suggests that a Nintendo-Wiiß exercise session provides an equivalent cardiovascular demand to a standard exercise session in an inpatient adult CF population. Greater enjoyment levels and lower fatigue levels reported during Nintendo-Wiiß training may have a positive influence on adherence to exercise. Further study into the long-term effects of NintendoWiiß training needs to be undertaken.

Conflict of Interest Nil.

Journal Compilation @ 2011 Asian Pacific Society of Respirology

\section{CITRATE'S EFFECTS ON AIRWAY ION TRANSPORT DIFFER FROM OTHER CALCIUM CHELATORS AND DICARBOXYLIC ACIDS}

P MIDDLETON ${ }^{1,2}, \mathrm{H}^{\text {HOUSE }}{ }^{1}$

${ }^{1}$ Ludwig Engel Centre for Respiratory Research, Westmead Millennium Institute, Westmead NSW, and '2University of Sydney at Westmead Hospital, NSW

Introduction Ion transport is important to maintain the airway epithelial surface, as shown by the disease cystic fibrosis (CF) which is characterized by decreased $\mathrm{Cl}^{-}$secretion and increased $\mathrm{Na}^{+}$absorption. We have previously shown that the CF airway can develop $\mathrm{Cl}^{-}$responses when the surface is nominally calcium free (Middleton et al. AJRCCM 2003; 168: 1223-1226).

Aim To determine the effects of citrate on the nasal potential difference (NPD) with and without amiloride pretreatment, and to compare these effects with other clinically relevant calcium chelators and dicarboxylic acids.

Methods NPD was measured using standard techniques (ERJ 1994; 7: 2050 ) in CF and non-CF subjects. The nasal PD response to citrate, oxalate, malate, succinate and fumarate (all $10 \mathrm{mM}$ ) was compared with the calcium chelators EDTA and EGTA.

Results Citrate decreased the basal NPD by $\sim 2 \mathrm{mV}$, but in the presence of amiloride, citrate increased the PD by $\sim 2 \mathrm{mV}$. With amiloride/low $\mathrm{Cl}^{-}$pretreatment, citrate increased NPD by 5-6 mV, which suggests that citrate increased $\mathrm{Cl}^{-}$secretion. In contrast, the other dicarboxylic acids and calcium chelators exhibited little response.

Conclusion The combination of these responses suggests that citrate exerts complex effects on airway ion transport, most likely dual effects of decreased $\mathrm{Na}^{+}$absorption and increased $\mathrm{Cl}^{-}$secretion.

Grant Support NH\&MRC and Cystic Fibrosis Australia.

Nomination Nil.

Conflict of Interest None. 
TP-080

\section{VALIDATION OF THE INTERNATIONAL PHYSICAL ACTIVITY} QUESTIONNAIRE IN ADULTS WITH CYSTIC FIBROSIS

TM RASEKABA ${ }^{1,3}$, BM BUTTON $^{1,2,4}$, JW WILSON ${ }^{2,4}$, AE HOLLAND $^{1,3}$ ${ }^{1}$ Dept. Physiotherapy, ${ }^{2}$ AlRmed, The Alfred Hospital, ${ }^{3}$ La Trobe University, and ${ }^{4}$ Monash University, Melbourne

Aim To assess the validity of the International Physical Activity Questionnaire (IPAQ) in CF adults by comparing energy expenditure measured by the $I P A Q$ versus the accelerometer.

Methods With ethics approval, suitable successive adult patients with CF attending the Alfred CF Outpatient Clinic were recruited. All participants wore an accelerometer (Actigraph GT1M) around the waist for 7 days of awake time, at the end of which, they completed the IPAQ. Criterion validity of the IPAQ was assessed by comparing the IPAQ weekly energy expenditure (EE) in kilocalories (kCal) with weekly EE (kCal) from the accelerometer using spearman correlations and Bland-Altman procedures.

Results Thirty participants ( $53 \%$ females) completed the assessment: mean $(\mathrm{SD})$; age $=29(7)$ years, $\mathrm{FEV} 1 \%$ predicted $=61(25) \%, \mathrm{BMI}=22(3) \mathrm{kg} \cdot \mathrm{m}^{-2}$. The median (range) EE: IPAQ = $3695(113,19573) \mathrm{kCal}, \mathrm{GT} 1 \mathrm{M}=1669$ $(355,5443) \mathrm{kCal}$. Spearman correlations of $\mathrm{FEV}{ }_{1} \%$ predicted with $\mathrm{EE}$ were GT1M EE $r=0.68, p<0.001$; IPAQ EE $r=0.28, p>0.05$. Correlation of the IPAQ EE with accelerometer EE was moderate $(r=0.46, p=0.010)$. There was a trend towards higher EE measured by the IPAQ than measured by the accelerometer (Wilcoxon signed ranks test: $z=-3.4, p=0.001$ ).

Conclusion The IPAQ underestimates physical activity for patients with lower energy expenditure activities and overestimates for those with higher energy expenditure activities in adults with CF. The IPAQ would be a useful screening tool for exercise prescription and monitoring of physical activity longitudinally, but more quantifiable methods for assessment such as the accelerometer should be used in research.

Conflict of Interest None.

\section{CLONAL COMPLEX PSEUDOMONAS AERUGINOSA IN HORSES: A MODEL OF CF INFECTION?}

TJ KIDD 1,2 , JS GIBSON 3 , S MOSS 3 , RM GREER ${ }^{3}$, RN COBBOLD ${ }^{3}$,

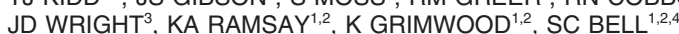

${ }^{1}$ Queensland Children's Medical Research Institute, ${ }^{2}$ Schools of Medicine and ${ }^{3}$ Veterinary Science, UQ, QId, and ${ }^{4}$ Thoracic Medicine, TPCH, QId, Australia

Infectious endometritis associated with Pseudomonas aeruginosa $(\mathrm{Pa})$ is an important equine disease resulting in reduced fertility and decreased foal drop. Previous typing studies of equine $P a$ report clonal heterogeneity, suggestive of sporadic acquisition, and small clusters of indistinguishable strains. Aim We performed molecular typing of a large sample of genital $P a$ isolates from horses in S-E Qld.

Methods Thoroughbred genital tract $P a$ isolates submitted to UQ Vet Diagnostic Lab during 2005-2009 (screening or infection suspected) were studied. ERIC-PCR fingerprint analysis was performed. Isolates producing indistinguishable fingerprints were allocated to the same ERIC-PCR type. MLST was performed on a subset of 24 isolates.

Results Overall, 2700 genital (clitoral or uterine) swabs from 2040 mares and 45 urethral fossa swabs from 18 stallions located on 16 stud farms were processed. Pa was identified in genital cultures from 93 of the 2040 (4.6\%) mares but from none of the 18 stallions. Six clusters involving $\geq 2$ mares were detected. Cluster-A was observed amongst isolates collected from $42(45 \%)$ mares from 13 studs and each year. Cluster-B isolates were present in 5 mares from 2 studs during 2005-2007. Clusters C-to-F each contained isolates from 2 mares from 1 or 2 studs.

Conclusions Overall, $57 \%$ of mares harbouring $\mathrm{Pa}$ had clonally related strains. However, we found no evidence of horizontal transmission between stallions. These data raise the possibility of transmission via environmental or other sources. Alternatively, specific strains may have trophism for the reproductive tract of horses. The finding of a dominant strain amongst thoroughbred mares in a geographic region has interesting parallels with recent evidence of the spread of highly prevalent clonal strains in cystic fibrosis clinics.

Supported by NHMRC, ACFRT.

Nomination Nil.

Conflict of Interest Nil.

\section{TP-081}

\section{PREVALENCE AND IMPACT OF INCONTINENCE IN ADULT MEN WITH CYSTIC FIBROSIS}

AT BURGE ${ }^{1}$, AE HOLLAND ${ }^{1,2,3}$, M SHERBURN $^{4}$, JW WILSON ${ }^{5,6}, \mathrm{~N} \mathrm{COX}^{3}$ TM RASEKABA ${ }^{1}$, R MCALEER ${ }^{7}, J_{\text {MORTON }}^{7}$, BM BUTTON ${ }^{1,5,6}$ ${ }^{1}$ Physiotherapy Dept, The Alfred Vic., ${ }^{2}$ Institute for Breathing and Sleep, Austin Health Vic., ${ }^{3}$ School of Physiotherapy, La Trobe University Vic., ${ }^{4}$ School of Physiotherapy, University of Melbourne Vic., ${ }^{5}$ Dept of Allergy, Immunology and Respiratory Medicine, The Alfred Vic., ${ }^{6}$ Faculty of Medicine, Nursing and Health Sciences, Monash University Vic., and ${ }^{7}$ Dept of Respiratory Medicine, Monash Medical Centre Vic.

Aim To investigate the prevalence and impact of incontinence in adult men with cystic fibrosis (CF) as compared with age- and sex matched control subjects.

Methods Men with CF were recruited through outpatient clinics and control subjects through advertisements to complete standardized questionnaires relating to respiratory symptoms, bladder and bowel function, mood and physical activity levels. Demographic data were collected from medical records for the CF group.

Results Seventy-four men with CF participated (mean (SD) age 29.9 (7.4) years). Forty-nine men volunteered as controls (33.5 (9) years), and were well matched in terms of physical activity levels. $11 / 74$ (15\%) in the CF group and $4 / 49(8 \%)$ in the control group had reported episodes of urine leakage. In the men with CF, there was no difference in lung function between men with episodes of leak and those with no history of leak (FEV $\%$ predicted $62(26) \%$ vs. $63(24) \%, p=0.943)$. Anxiety levels were higher in men from both groups with episodes of leak compared to those with no history of leak (Hospital Anxiety and Depression anxiety score 9.3 (4.1) vs. 4.6 (3.8), p < 0.001). Depression scores were also higher in men with episodes of leak compared to those with no history of leak (6.4 (4.4) vs. 2.1 (2.9), $p<0.001)$.

Conclusions Urinary incontinence in men with CF is not associated with disease severity, as measured by lung function. Anxiety and depression levels were higher in men with leakage of urine.

Conflict of Interest No.

\section{BONE HEALTH IN CHILDREN WITH CYSTIC FIBROSIS}

TP-083

V SIMOVIK ${ }^{1}$, M JAKSIC ${ }^{1,2}$, J TATE1 ${ }^{1}$, T CUNDY², CA BYRNES, 1,2

${ }^{1}$ Starship Children's Hospital, Auckland, New Zealand, and ${ }^{2}$ Department of Paediatrics, University of Auckland

Background With increasing life expectancy, the Cystic Fibrosis Foundation (USA) annual report 2008 highlighted the emergence of bone disease in patients with cystic fibrosis (CF).

Aim To investigate the bone mineral status of children and adolescents with $\mathrm{CF}$ and to explore the relationship between bone mineral density (BMD) and anthropometric and clinical parameters including height, body mass index (BMI), lung function tests and vitamin D levels (25-hydroxyvitamin D) in the CF centre at Starship Children's Hospital, New Zealand.

Methods BMD of the lumbar spine was assessed by dual X-ray absortiometry between January 2007 and December 2009. The results of 35 subjects with CF (21 males) with a mean age of 14.6 years (range $10-18.8$ years) were collected. Anthropometric data (height, BMI), forced expiratory volume in 1 second as percent predicted $\left(\% F_{E V}\right)$ and vitamin levels were assessed and related to $B M D$.

Results BMD in our subjects was low in $25.7 \%$ and very low in $8.6 \%$ when adjusted for age, sex and height (difference in BMD g/cm ${ }^{2}$ in the lumbar spine L1-L4). There was a strong positive relationship between the lumbar areal BMD (aBMD) and BMI Z scores $(p<0.0001)$, aBMD and \% FEV 1 z scores ( $p$ $<0.003$ ), and aBMD Z scores and vitamin D levels $(p<0.03)$.

Conclusions BMD was normal in the younger and well-nourished subjects with normal or mild reduction of $\mathrm{FEV}_{1}$. Low BMD appeared to evolve during adolescence with decreasing $\mathrm{BMI}$ and reduction in lung function. This will lead to ongoing bone disease in early adulthood. It is a further indication to maintain optimal nutritional status and maximize lung health.

Conflict of Interest No.

Journal Compilation (C 2011 Asian Pacific Society of Respirology 


\section{NUTRITION IN ADULTS WITH CYSTIC FIBROSIS (CF):} 10 YEARS ON - HAS IT CHANGED?

\section{K HERD, AG MATSON, SC BELL}

Adult CF Centre, The Prince Charles Hospital, Brisbane, Australia

Malnutrition in $\mathrm{CF}$ is associated with poorer pulmonary function and is an independent risk factor of survival.

Aim To compare the nutritional status of the adults attending an Adult CF Centre in 2009 with 1999.

Method Retrospective audit of 260 patients (48 excluded, incomplete data) including demographics, nutritional status, pancreatic enzyme replacement therapy (PERT) usage, glucose tolerance and dietetic review.

Results The mean age of the clinic population increased from 26.8 to 29.8 years. Mean (SD) BMl increased from $1999\left(21.1 \pm 3.0 \mathrm{~kg} / \mathrm{m}^{2}\right)$ to 2009 $(22.5 \pm 3.8)(p=0.0017)$. In $2009,89 \%$ of the clinic population was taking PERT with a mean dose of $4315 \pm 2782 \mathrm{IU}$ lipase $/ \mathrm{kg} /$ day. The proportion of patients with abnormal glucose tolerance has increased from $12 \%$ to $26 \%$ (p $=0.004$ ). Oral supplement use has increased from $26 \%$ to $45 \%$, yet enteral feeding remained stable $(12 \%-1999,8 \%-2009)$. This occurred during period of increased annual dietetic review of the patients attending the clinic from $73 \%$ in 1999 to $90 \%$ in 2009 ( $p=0.0001)$.

Discussion Over a 10-year period, an improvement in mean BMI reflects improvement in nutritional status. Prevalence of abnormal glucose tolerance has increased; this is likely due to commencing a screening program (2000). Use of oral supplements has increased and is higher than identified in the recent DAA survey of nutrition practices of CF Dietitians (18\%). Annual review by the CF Dietitian has increased despite a twofold increase in the CF population may be attributable to a stable and experienced workforce. Current service provision of 1.1 FTE per 260 patients is $55 \%$ of the recommended staffing level (2.0FTE) as per Cystic Fibrosis Standards of Care, Australia 2008.

Supported by Nil.

Nomination Nil.

Conflict of Interest Nil.

POSTER WITHDRAWN

\section{EXTENDED CULTURE ADDS LITTLE TO THE IDENTIFICATION OF POTENTIAL PATHOGENS IN ADULT PATIENTS WITH STABLE NON-CYSTIC FIBROSIS BRONCHIECTASIS}

\author{
A ABBOTT ${ }^{1}$, E CHEUNG ${ }^{2}$, L MORGAN ${ }^{1}$ \\ ${ }^{1}$ Respiratory Medicine Concord Repatriation General Hospita, NSW 2050 \\ and ${ }^{2}$ Infectious Diseases Concord Repatriation General Hospital NSW 2050
}

Aim To characterize the microbial colonization of a group of stable adults with non-CF bronchiectasis using an extended culture protocol.

Methods Sputum was collected over an 18-month period from clinically stable patients. Standard semi-quantitative bacterial culture was extended to 7 days with the addition of fungal and mycobacterial culture as routine.

Results 61 specimens of spontaneously expectorated sputum were collected from 28 patients; mean age 62 years (21-87 years); mean (SD) FEV 1 FVC ratio $63 \%$ (22\%); $21 / 28$ never smokers; $19 / 28$ on inhaled or oral corticosteroids. The bacteria identified were $P$. aeruginosa (21\% of specimens), $H$. influenzae $(18 \%), H$. parainfluenzae $(5 \%)$, Acinetobacter baumanii $(2 \%)$, Enterobacteriaceae (6\%). Commensals only were identified in $43 \%$ of specimens. Fungi included Candida species (20\%), Aspergillus fumigatus (5\%) and Penicillium species $(2 \%)$. Non-tuberculous mycobacteria (NTMB) were grown in $11 \%$ of specimens: M. gordonae $(7 \%), M$. intracellulare $(2 \%)$ and $M$. lentiflavum $(2 \%)$. The NTM identified were all considered non-pathogenic. Only the mycobacteria were identified after day 2.

Conclusion Microorganisms with potential pathogenicity are frequently identified in adult patients with non-cystic fibrosis bronchiectasis who are not experiencing an acute exacerbation. All these organisms were identified using a standard short culture protocol. The extended regimen, which was costly, did not identify any unusual or unexpected pathogens. It was rare for patients to be colonized with fungi. This study suggests there is limited value in requesting extended culture for bacterial pathogens, including looking for fungi or NMTB in this stable patient group as this adds little to the empiric antibiotic choice for infective exacerbations.

Conflict of Interest None.
DETECTION OF VIRAL AND BACTERIAL RESPIRATORY PATHOGENS IN CHILDREN WITH CYSTIC FIBROSIS

S STELZER-BRAID ${ }^{1,2}$, H ALSUBIE $^{3}$, A NEILSEN ${ }^{3}$, H JOHAL $^{1}$, A STELLER ${ }^{1}$, ER TOVEY ${ }^{4}, K_{\text {MCKAY }}^{3}$, P VAN ASPEREN ${ }^{3}$, WD RAWLINSON ${ }^{1,2}$

${ }^{1}$ Virology Research Laboratory, Prince of Wales Hospital, NSW, Australia,

${ }^{2}$ School of Medical Sciences, The University of New South Wales, NSW, Australia, ${ }^{3}$ Respiratory Department, Children's Hospital Westmead, NSW, Australia, and ${ }^{4}$ The Woolcock Institute of Medical Research, The University of Sydney, NSW, Australia

Introduction Respiratory infections are of fundamental importance in determining the morbidity and mortality associated with cystic fibrosis (CF) as such infections can lead to progressive and fatal obstructive lung disease. Using polymerase chain reaction (PCR) to detect such infections has advantages over previous studies that used relatively insensitive traditional detection methods and could have underestimated viral prevalence.

Methods Viral and bacterial multiplex PCRs were developed for detection of respiratory pathogens important for children with CF. Nasal brush samples were collected from CF patients who were symptomatic or asymptomatic for acute respiratory illness $(n=56)$. Sputum and exhaled bioaerosols via a novel mask sampler were collected from a subset $(n=16)$.

Results As expected, almost all $(81 \%)$ sputum samples were positive for bacteria. Detection of bacteria in the upper respiratory tract was lower (12.5\%). Data from nasal samples indicated strong association of viral pathogen presence, particularly rhinovirus, with exacerbation of disease. Results also showed good evidence for rhinovirus infection in the lower respiratory tract. The novel mask sampler is promising as a non-invasive sampling tool.

Conclusions Our results demonstrate the importance of pathogens in exacerbations. Early detection and understanding the development of bacterial and viral infections in CF patients is important in clinical decision-making as more and better antiviral and antibiotic agents become available.

Supported by NH\&MRC \#571380.

Conflict of Interest $\mathrm{No}$. 
INTERVENTIONAL PULMONOLOGY SIG: POSTER SESSION

\section{TP-088

\begin{abstract}
UNEXPECTED HIGH VIRAL YIELD FROM BAL IN ACUTE PNEUMONITIS IN IMMUNOSUPPRESSED HAEMATOLOGY
\end{abstract} PATIENTS \\ DN DELLER, DI FIELDING \\ Respiratory Medicine, The Royal Brisbane Hospital, Queensland, Australia}

Aim To determine the factors affecting microbiological yield from bronchoalveolar lavage (BAL) in patients with suspected pulmonary infection and haematological malignancy or following stem cell transplantation at a tertiary bone marrow transplant centre.

Methods A retrospective 12-month audit of patients with pulmonary infiltrates or febrile neutropenia with haematological malignancy or post-stem cell transplant who underwent BAL for microbiological diagnosis. Data were obtained on microbiological yield, radiographic appearances, current antimicrobial therapy, the presence and duration of neutropenia and complication rate.

Results Of the 48 BAL procedures performed, a clinically significant microbiological result was obtained in $33 \%$ of cases $(16 / 48)$. Of these positive results, 31\% (5/16) were exclusively viral pathogens, $19 \%(3 / 16)$ were fungal, $25 \%(4 / 16)$ were bacterial and polymicrobial infection was observed in $25 \%$ $(4 / 16)$ of cases. A high proportion of patients had commenced anti-microbial treatment empirically, with $88 \%(42 / 28)$ receiving broad spectrum antibacterial treatment and $56 \%(27 / 48)$ receiving treatment doses of antifungal agents prior to bronchoscopy. In $23 \%$ (11/48), the results of the BAL changed the patients therapy. The presence and duration of neutropenia or radiological appearances were not reliable discriminators of specific infective aetiologies. Complication rates were low and included fevers in 25\% (12/48), hypoxia 8\% (4/48), small volume haemoptysis in $2 \%(1 / 48)$, atrial fibrillation in $2 \%(1 / 48)$ and pneumothorax in $2 \%(1 / 48)$.

Conclusion Whilst BAL remains a safe and important tool in establishing a microbiological diagnosis in immunosuppressed patients with pulmonary infiltrates, a clinically significant yield and changes to patient treatment occur in the minority of cases. Clinicians should have a high degree of suspicion of viral infective aetiology when treating this population of patients.

Conflict of Interest No.

\section{INTERCOSTAL CATHETER TREATMENT OF PNEUMOTHORAX} AND PLEURAL EFFUSION: AN AUDIT

GW DON 1 , PJ WU 1 , JR WHEATLEY ${ }^{1,2,3}$, TD ROBINSON ${ }^{1,2}$

${ }^{1}$ Department of Respiratory \& Sleep Medicine, Westmead Hospital, ${ }^{2}$ Ludwig Engel Centre for Respiratory Research, Westmead Millenium Institute, and ${ }^{3}$ University of Sydney at Westmead Hospital, Westmead, NSW 2145, Australia

Aim To examine the outcomes and complications of intercostal catheter (ICC) treatment of pneumothoraces (primary (PP) and secondary (SP)) and effusions (malignant (ME) and parapneumonic (PE)).

Methods Retrospective review of all ICCs in admitted patients in a respiratory unit over 15 months. Data collected included type of pneumothorax or effusion, ICC type, insertion details, complications (major and minor) and outcome (success defined as resolution of pneumothorax or effusion with single tube insertion).

Results 88 patients required ICC treatment. Forty-six ICCs were used in 38 patients with pneumothorax: PP 18; SP 14; iatrogenic 5; hydropneumothorax 1. Complication rate was $45 \%(12 \%$ major) and was significantly less in PP $(35 \%)$ compared with SP $(60 \%), p<0.05$, chi-square. Success rate for pneumothorax ICC drainage was $44 \%$ (significantly higher for PP (72\%) compared with SP $(20 \%), p<0.05)$. Fifty-eight ICCs were used in 50 patients with pleural effusions: ME 35, PE 11, other 4. Complication rate was 57\% (13\% major) and was significantly higher in ME $(67 \%)$ compared with PE $(36 \%), p<0.05$. Success rate for effusion ICC drainage was $58 \%$ (significantly less in ME $(49 \%)$ compared with PE $(81 \%), p<0.05)$. Small bore ICCs (gauge $<16 \mathrm{Fr}$ ) were used for $91 \%$ of pneumothoraces and $66 \%$ of effusions. Tube size did not significantly influence complication or success rate for either pneumothoraces or effusions.

Conclusions Compared with PP, ICC treatment of SP was less successful and more likely to be associated with complications. Similarly, compared with $\mathrm{PE}$, intervention for ME with ICC was less successful and had a higher complication rate. We conclude that ICC intervention is most successful for PP and $\mathrm{PE}$, and speculate that SP and ME should have early surgical intervention.

Supported by Nil.

Nomination Nil.

Conflict of Interest No.

\section{AUDIT OF LARGE SPONTANEOUS PNEUMOTHORAX MANAGEMENT}

U DIBIA 1 , D SMITH ${ }^{2,3}, \mathrm{P} \mathrm{MASEL}^{2,3}$

Departments of ${ }^{1}$ Internal and ${ }^{2}$ Thoracic Medicine, The Prince Charles Hospital, Brisbane Qld, and ${ }^{3}$ School of Medicine, University of Queensland, Brisbane, Qld

Introduction Spontaneous pneumothorax is a common condition. Current management guidelines recommend large pneumothoraces are managed by primary intercostal catheter insertion. We report a single centre's experience in the management of large spontaneous pneumothorax.

Methods Retrospective audit of cases of spontaneous pneumothoraces managed at the Prince Charles Hospital between 1 January and 31 December 2009. Patient demographics, co-morbidities, presenting symptoms, examination findings, radiology, management and complications were reviewed. Results Forty-two patients (36 male, 6 female) experienced 61 episodes of spontaneous pneumothorax. Chest pain and dyspnoea were the most commonly reported symptoms (50) $82 \%$. There were forty-two (69\%) episodes of large pneumothorax ( $\geq 20 \%$ of hemithorax). Management of large pneumothoraces consisted of: observation, (15) Seldinger ICC (17) and Large bore ICC (10). Complications occurred in three patients with Seldinger ICC (2 vasovagal, 1 hydro-pneumothorax) compared to none with large bore ICC. Outcomes were similar for patients managed by observation compared to ICC insertion. All recurrent cases $(26 \%)$ were referred for consideration of surgical pleurodesis.

Conclusion Patients with large pneumothorax managed by observation recovered similarly to those treated with ICC, suggesting a higher threshold for ICC insertion should be considered in the future.

Grant Support Nil.

Conflict of Interest No.

\section{PILOT STUDY OF PLEURAL ULTRASOUND TRAINING ASSESSMENT TOOL AND ULTRASOUND MANIKIN}

\section{S ERIGADOO ${ }^{1}$, D MGRATH ${ }^{2}, \mathrm{~K}$ STEINKE ${ }^{2}$, J COUCHER ${ }^{3}, \mathrm{~K} \mathrm{DAVIES}^{4}$, D FIELDING ${ }^{1}$}

${ }^{1}$ Thoracic Medicine Royal Brisbane Hospital, Qld, Australia, ${ }^{2}$ Radiology Department, Royal Brisbane Hospital, ${ }^{3}$ Radiology Department, Princess Alexandra Hospital, Qld, and ${ }^{4}$ Australian Institute of Ultrasound, Gold Coast, Qld, Australia

Aim A pilot study of an instrument of pleural ultrasound training in thoracic physicians after a pleural ultrasound course. The instrument was tested for inter-observer agreement and also its ability to be used in a patient compared to a dedicated manikin.

Methods All chest physicians (4) were novices in ultrasound and underwent a dedicated 3-day training course in pleural ultrasound at The Australian Institute of Ultrasound. They were assessed 3 months later by 2 radiologists and one senior ultrasonographer using a specially designed pleural ultrasound training assessment tool (USGT-SAT) on both a subject with pleural effusion and a dedicated ultrasound manikin.

Results The mean scores, out of a maximum of 100 , obtained by the each of the 4 participants for the manikin were 95.0, 87.7, 89.3 and 84.3 , respectively, while the scores for the patient was $87.3,85.0,94.0$ and 86.7 , respectively. The mean scores of the participants as a group for manikin were $89 \pm$ 7.2 and for the patient as $88.3 \pm 8.11$. There was general agreement between the 3 examiners with mean combined participant scores of $91.5,88.8$ and 87.0 in the manikin, respectively, and mean score of $95.8,81.3$ and 87.8 in the patient.

Conclusions This pilot study shows ranges of scores for design of future validation studies of the USGT-SAT. Test performance by the chest physicians after a short course in pleural ultrasound was generally good and results for the use of the manikin as an alternative to patients in pleural ultrasound training are encouraging. Further studies with larger sample size are required.

Supported by Nil.

Nomination Nil.

Conflict of Interest No.

Journal Compilation (C 2011 Asian Pacific Society of Respirology 


\section{FLEXIBLE BRONCHOSCOPY TO INTERVENTIONAL PULMONOLOGY: A NURSING PERSPECTIVE}

\section{SM FOWLER, L HOLSWORTH, TJ WILLIAMS}

Department of Allergy, Immunology and Respiratory Medicine, The Alfred, Melbourne, Vic., Australia

Since the first commercial availability in 1968, flexible bronchoscopy has evolved from a simple 'look see' procedure to a more complex multifaceted one. Today, flexible bronchoscopy is a tool used for diagnostic procedures, surveillance, delivery of therapy and clinical trials. Increasingly, it involves utilizing expensive purpose built equipment in complex diagnostic procedures. This evolution requires a specific knowledge base and skill set to safely perform the procedure and care for the equipment. This now mandates additional training by nursing and medical staff to develop and maintain the required skills. Medical staff now rely on their nurses to assist in the full range of procedures. Thus, the nurses must keep abreast of modern trends and techniques. The modern bronchoscopy suites team is an integrated one, with specific roles, defined to each member. The procedures performed will reflect local needs and expertise.

Just as bronchoscopy has evolved into the speciality of interventional pulmonology, so must bronchoscopy suite nursing be accepted as a specialized area of nursing with a credentialed 'Special Interest Group' to promote, educate and develop the subject as more therapeutic and diagnostic procedures evolve. This will allow nurses involved in bronchoscopy to be respected, recognized and accepted for their unique knowledge and abilities.

Conflict of Interest Nil.

\section{TRANSTHORACIC PNEUMONOSTOMY - EFFECT ON LUNG VOLUME AND MECHANICS}

IT LING ${ }^{1,2}$, B SINGH ${ }^{1}$, MJ PHILLIPS 2

Departments of ${ }^{1}$ Pulmonary Physiology and ${ }^{2}$ Respiratory Medicine, Sir Charles Gairdner Hospital, WA, Australia

Background Transthoracic pneumostomy (TP) is a novel treatment for patients with severe emphysema that aims to deflate the lung and improve function.

Aim To assess the effect of unilateral TP on the volume of each lung and mechanical properties of the lungs.

Methods Subjects were recruited for a multicentre trial of TP (see ACTRN $12608000408381)$. In parallel with the main protocol, we measured (1) the volume of each lung in the erect posture using PA and lateral CXRs with the methods of Pierce et al. Thorax 1979 and in the supine posture using CT volumetric analysis; (2) dynamic hyperinflation from the change in inspiratory capacity (IC) with exercise, and (3) the static volume-pressure relationship of the lung by oesophageal manometry. Minimum follow-up was 6 months.

Results In the six subjects recruited, compared to plethysmography, lung volume was overestimated by CXR (mean difference $+3.4 \%$, range -16.6 to +17.3 ) and underestimated but more closely correlated by CT (mean difference $-5.5 \%$, range -14.9 to -1.9 ). Based on $\mathrm{CT}$, the volume of the treated lung decreased in all patients after TP (mean $-17.6 \%$, range -6.3 to -28.1 ) whilst that of the untreated lung did not change (mean $-1.4 \%$, range -6.3 to +5.1 ). In 5 patients with available data, TP reduced dynamic hyperinflation during exercise (mean $-180 \mathrm{~mL},-8.4 \%$ of IC, range $+0.7 \%$ to $-17.9 \%$ ). Lung mechanics were performed in 4 patients. Low lung elastic recoil prior to TP and an increase in elastic recoil after TP were associated with greater reductions in lung volume and greater improvements in exercise tolerance.

Conclusions Supine chest CT provided reasonably accurate estimates of plethysmographic lung volume. Unilateral TP deflated the lung and there was no evidence of significant compensatory hyperinflation of the contralateral lung. TP also reduced dynamic hyperinflation. Measurement of lung elastic recoil may help select patients who are likely to benefit from TP.

Support and Conflict of Interest Nil.

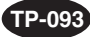

\section{COMPLICATIONS OF SMALL BORE INTERCOSTAL CHEST CATHETERS}

S GUPTA, JP WILLIAMSON, P BUCHANAN, S SRIKANTHA, PW COLLETT Department of Respiratory Medicine, Liverpool Hospital, NSW, Australia

Background and Objectives Limited data are available on complications associated with small bore intercostal chest catheters (ICC) inserted via the Seldinger technique.

Methods We performed a retrospective chart review of all adult patients who had an ICC over a 24-month period within a tertiary hospital respiratory service. We noted patient demographics, details surrounding chest drain insertion including image guidance and subsequent inpatient events.

Results Over a 24-month period, there were 102 small-bore ICC insertions, of which 28 were image-guided. Mean patient age was 58 years, males com prised 69/102. Forty drains were inserted for pneumothoraces, 25 for malignant effusions, 18 for parapneumonic effusions, 6 for transudates and 13 fo undiagnosed exudative effusions. Mean duration of drainage was 4.8 days There were no life-threatening complications. Three of the chest drains fell out and 7 became blocked. Six pneumothoraces were noted, all following insertion without direct image guidance; none required further intervention. Local infection occurred in 1 patient. Insertion details were not documented in 9 patients. Conclusion Insertion of small-bore ICCs via the Seldinger technique appears to be a safe method of draining pneumothoraces and pleural effusions. Image guidance may reduce complication rate of this procedure. Documentation of drain insertions could be improved.

Conflict of Interest $\mathrm{Nil}$

Journal Compilation (C) 2011 Asian Pacific Society of Respirology

\section{A REVIEW OF OUTCOMES FOLLOWING PLEURAL ASPIRATION}

G PALNITKAR ${ }^{1}$, M HIBBERT $^{1}$, A GILL $^{2}$, B HARRIS

${ }^{1}$ Department of Respiratory Medicine, and ${ }^{2}$ Department of Anatomical

Pathology, Royal North Shore Hospital, Sydney, NSW, Australia

Rationale Pleural effusions are frequently encountered in clinical practice, and often require aspiration for diagnostic and/or therapeutic purposes. Use of radiological guidance varies, despite current guidelines recommending routine use of ultrasound. Furthermore, concerns exist regarding the downskilling of thoracic medicine trainees due to the increased use of interventional radiology. As a precursor to developing a procedural pleural ultrasound service, we performed a retrospective case review of our current practice.

Methods Patients who had pleural fluid sent to pathology between January 2008 and December 2009 were identified on an existing database. Patient records were reviewed and details regarding the drainage procedure and outcomes were recorded. Information on patient location, method of procedure and performing clinician were also collected.

Results To date, 22 pleural fluid aspirations in 21 patients have been identified. Overall, $68 \%$ of aspirations were carried out on the ward and $18 \%$ in the radiology department. Two procedures occurred in the endoscopy suite on outpatients, and one in the emergency department. Fifty percent of procedures were performed using an intravenous cannula for drainage and $45 \%$ utilized a pigtail catheter. All procedures occurring in the radiology department were performed under ultrasound guidance by a radiologist or radiology registrar. Of the remaining procedures, $56 \%$ were performed by medical registrars and $61 \%$ were performed with ultrasound marking. Six complications occurred following 5 procedures: 4 pneumothoraces, 1 vasovagal and 1 tube blockage. There were significantly more pneumothoraces in patients who did not have an ultrasound marking ( 3 of 7 without marking, 1 of 15 with marking, $p=0.03$ ). None of the complications required further intervention.

Conclusion These preliminary data suggest ultrasound marking significantly reduces pneumothorax incidence, supporting the establishment of a pleural ultrasound service. This is likely to have the added benefit of improved training for thoracic medicine trainees.

Conflict of Interest 
TP-096

\section{POSTURE INFLUENCES PATIENT COMFORT, COUGH RATE} AND SEDATIVE REQUIREMENT DURING BRONCHOSCOPY

IT LING, F PICCOLO, SA MULRENNAN, MJ PHILLIPS

Department of Respiratory Medicine, Sir Charles Gairdner Hospital, WA Australia

Aim To investigate differences between semi-recumbent and supine posture in terms of cough rate, degree of oxygen desaturation, oxygen supplementation, increase in pulse rate and sedative use during the initial phase of bronchoscopy.

Methods Consecutive patients $(n=69)$ undergoing diagnostic bronchoscopy at an endoscopy unit were recruited for this observational cohort study. The posture was determined by the bronchoscopist's usual practice. Patient age, gender, \% predicted $\mathrm{FEV}_{1}$ and $\mathrm{FVC}$, indication, pulse and oxygen saturation were recorded. The initial phase was defined as the time from bronchoscopy insertion to visualization plus lignocaine instillation of both distal main bronchi. Cough rate, peak pulse, nadir oxygen saturation ( $\mathrm{SpO} 2)$, range of oxygen supplementation and sedation use during the initial phase were recorded. A post-procedure questionnaire was administered to the patient and the attending nurse.

Results 36 patients had bronchoscopy in the semi-recumbent posture and 33 in the supine posture. Three of 5 bronchoscopists performed in both postures. There were no significant differences in age, gender, smoking status and spirometry between the two groups. The semi-recumbent postures resulted in significantly less cough rate (mean (SD) 3.6 (2.3) vs. $6.3(4.8)$ coughs $/ \mathrm{min}, \mathrm{p}=0.004)$ and less fentanyl use $(60$ (16) vs. $76(22) \mathrm{mcg}, \mathrm{p}=$ $0.001)$ in the initial phase. There were no significant differences in the nadir $\mathrm{SpO} 2$, fall in $\mathrm{SpO} 2$, oxygen supplementation or increase in pulse rate between the two groups. Nurse perception of patient discomfort was lower in the semirecumbent position (23 (21) vs. 39 (28) $\mathrm{mm}$ on $100 \mathrm{~mm}$ visual analogue scale, $p=0.01$ ), and there was a trend towards less patient-perceived cough during the procedure in the semi-recumbent group (28 (25) vs. $40(28) \mathrm{mm}$ on $100 \mathrm{~mm}$ visual analogue scale, $p=0.06$ )

Conclusions Bronchoscopy performed in the semi-recumbent posture results in less cough and sedative requirement, and may improve patient comfort.

Support and Conflict of Interest Nil.

\section{UTILITY OF BRONCHOSCOPY IN PATIENTS WITH HAEMATOLOGICAL MALIGNANCY AND NEW LUNG INFILTRATES}

\section{S SRIKANTHA, JP WILLIAMSON, GB MARKS, S GUPTA, PW COLLETT Department of Respiratory medicine, Liverpool Hospital, Liverpool, NSW,} Australia

Introduction Pulmonary infiltrates in immunocompromised patients with haematological malignancy have a diverse aetiology and are a major source of morbidity. A specific diagnosis and targeted therapy may optimize outcomes and reduce the cost of treatment. The diagnostic value of fibreoptic bronchoscopy (FOB) and the influence of timing of the procedure are unclear.

Aim To determine the yield of FOB, its impact on antibiotic therapy and the influence of early vs late timing in this patient population.

Methods We conducted a retrospective review of immunosuppressed patients with underlying haematological malignancy and new pulmonary infiltrates who underwent FOB over a 30-month period. The outcomes of early ( $E B, \leq 2$ days from initial respiratory consultation) and late ( $L B, \geq 3$ days) FOB were compared using Fisher's exact test.

Results Thirty-eight FOBs, including 6 bronchial or transbronchial biopsies, were performed in 33 patients (males 21). There were 19 patients who received $\mathrm{EB}$ and 19 who received $\mathrm{LB}$. A specific diagnosis was obtained from 13 procedures (34\%), including 11 infections (6 in EB vs. 5 in $L B, p=1.0$ ) and 4 non-infective diagnoses ( $1 \mathrm{~EB}$ vs. $3 \mathrm{LB}, \mathrm{p}=0.6$ ) based on histology. FOB findings from 17 procedures $(45 \%)$ (11 EB vs. $6 \mathrm{LB}, p=0.2$ ) resulted in modification of antibiotic therapy. There were no procedure-related severe complications.

Conclusions FOB is a useful diagnostic procedure which influences diagnostic and therapeutic decisions in this patient group. Although early procedures tended to be more likely to change antibiotic therapy than late procedures, the difference was not significant.

Conflict of Interest None.
TP-097

\section{CAPSULE BRONCHOSCOPY: AN UNUSUAL FOREIGN BODY IN} THE LUNG

G SIMPSON

Cairns Base Hospital, Cairns, QId, Australia

Capsule endoscopy is increasingly performed in gastroenterology to investigate possible small intestinal bleeding. The capsule endoscope is swallowed and then takes photographs every 4 seconds for 8 hours during its transit through the gastrointestinal tract. The images are downloaded by a radio link and the capsule is then passed normally and disposed of. In the present case, the capsule endoscope was inhaled and lodged in the bronchus intermedius. This was only recognized when the images from the capsule download were examined. Removal of the capsule was effected with a fibreoptic bronchoscope using an ERCP balloon and Roth basket. This is believed the only capsule bronchoscopy so far reported. Capsule endoscopes are large $(23 \mathrm{~mm}$ $\times 11 \mathrm{~mm}$ diameter) and smooth. This case report shows the images from the capsule endoscope and describes the methods necessary to remove this unusual foreign body from the lung.

Support Nil.

Conflict of Interest Nil.
BRONCHIAL ARTERY EMBOLIZATION AS A DEFINITE TREATMENT MODALITY IN A PATIENT WITH MASSIVE HAEMOPTYSIS

ZA USMANI, A ROY, R SEBBEN, SI AZIZ, I NIKITINS

Department of Respiratory Medicine, The Queen Elizabeth Hospital, SA, and Department of Radiology, The Queen Elizabeth Hospital, SA

Introduction Conservative management of massive haemoptysis carries a mortality rate of $50 \%-100 \%$. Mortality rates for surgery performed for massive haemoptysis range from $7.1-18.2 \%$ and it increases up to $40 \%$, when the surgery is undertaken as an emergency procedure.

Case A middle-aged woman with bronchiectasis presented with eight episodes of $30-50 \mathrm{~mL}$ of frank haemoptysis. CT chest revealed grossly enlarged right bronchial artery and bronchoscopy revealed several abnormal submucosal vascular channels in the bronchus intermedius, correlating with the $C T$ findings. Right bronchial artery embolozation (BAE) was performed with a record of 18 Micro coils, the procedure was uncomplicated. At 6 months followup, the patient did not report any further episodes of haemoptysis and follow-up CT chest and bronchoscopy revealed shrinkage of the abnormal right bronchial artery and disappearance of abnormal vascular channels.

Conclusion In expert hands and in selected patients, BAE could be considered as a safer option for definite treatment of massive haemoptysis.

Journal Compilation @ 2011 Asian Pacific Society of Respirology 
RESEARCH BRONCHOSCOPIC BIOPSY IN ADULT BRONCHIECTASIS SUBJECTS IS ASSOCIATED WITH SIGNIFICANT BLEEDING RISKS

\author{
DJ SERISIER ${ }^{1,2}$, M MARTIN $^{1}$, SD BOWLER $^{1}$ \\ ${ }^{1}$ Respiratory Medicine, Mater Adult Hospital, QId, Australia, and ${ }^{2}$ School of \\ Medicine, University of Queensland, QId, Australia
}

Background Bronchoscopy with endobronchial biopsy (EB) is now an integral component of the research evaluation of airway diseases. There are no published safety data for EB in adult non-CF bronchiectasis.

Methods A subgroup of subjects enrolled in the Bronchiectasis and Low Dose Erythromycin Study (BLESS) a randomized controlled trial of long-term prophylactic erythromycin (ANZCTRN12608000460303) underwent bronchoscopy with bronchoalveolar lavage $(\mathrm{BAL})$ and $\mathrm{EB}$ performed by a single operator.

Results Ninety-nine bronchoscopies were performed (BAL alone in 10) in 41 subjects. Of 89 procedures with EB, 4 (4.5\%) were associated with very significant bleeding $(>100 \mathrm{~mL}$ either at time of EB or several days post-procedure) and a further $3(3.4 \%)$ with immediate moderate bleeding (25-50 mL) One subject had a history of prior significant haemoptysis. In the four subjects with very significant bleeding, immediate bleeding of $>150 \mathrm{~mL}$ occurred in 2 subjects, $40 \mathrm{~mL}$ in one subject and $25 \mathrm{~mL}$ in one. Immediate bleeding was controlled uneventfully. Three of the 4 subjects subsequently developed significant haemoptysis ( $>100 \mathrm{~mL}) 4$ to 6 days post-bronchoscopy without intervening haemoptysis, with one subject developing massive haemoptysis $(>300 \mathrm{~mL})$ on day 4 post-bronchoscopy. Further research EBs were ceased. In one of the 3 subjects with 'delayed rebleeding', repeat bronchoscopy confirmed the biopsied lobe as the bleeding site. Haemoptysis settled in all 3 subjects within 36 hours with simple conservative measures.

Conclusions In contrast to the experience in asthma and COPD, research EB in adults with non-CF bronchiectasis is associated with a significant risk of bleeding, of potentially life-threatening magnitude in $4.5 \%$ of cases. Of particular concern was the observation of sudden onset delayed rebleeding developing up to 6 days post-EB in spite of early local control. Histopathological evaluation will clarify the potential contributions of airway wall vascularity and inflammation to these events.

Conflict of Interest No.

LUNG CANCER SIG: POSTER SESSION

TP-101

DETERMINANTS OF LATENCY PERIODS OF LUNG CANCER (LC) AND MALIGNANT MESOTHELIOMA (MM) IN FORMER WORKERS AND RESIDENTS EXPOSED TO CROCIDOLITE AT WITTENOOM, WESTERN AUSTRALIA

P ABOAGYE-SCARFO', A REID ${ }^{1,2}, \mathrm{~N}^{1}$ OLSEN ${ }^{1}$, L SAMUELS $^{1}$, P FRANKLIN ${ }^{1}, N$ DE KLERK ${ }^{1,3}$, AW MUSK ${ }^{1,4}$

${ }^{1}$ School of Population Health, UWA, Crawley, WA, ${ }^{2}$ Centre for Medical Research, UWA, Crawley, WA, ${ }^{3}$ Telethon Institute of Child Health Research, Subiaco, WA, ${ }^{4}$ Sir Charles Gairdner Hospital, Nedlands, WA

Aim To estimate the determinants of latency periods of LC and MM in people exposed to blue asbestos (crocidolite) mined and milled at Wittenoom, WA from 1943 to 1966 . The town closed in 2006.

Subjects Workers and residents cohorts were established from employment records, electoral rolls, medical fund contributions and school and medical attendance records. Five hundred eight LCs and $439 \mathrm{MMs}$ with exposure estimates were documented between 1953 and May 2010.

Methods Incident cases were obtained from the Australian and WA Mesothelioma and Cancer Registries and Death Registries. Exposure was calculated using measures of dustiness in the industry and the town for the period of employment or residence of each case. Latency (time from first exposure to diagnosis) by sex, age, smoking status, exposure variables and worker or resident status was estimated. Multivariate linear regression modelling examined the determinants of latency.

Results The mean latency periods of $32.4(S D=11.4)$ years for $L C$ and 36.8 $(S D=9.2)$ years for MM have increased linearly. Increased duration of exposure was associated with reduced latency for MM after adjustment for age at first exposure and age at diagnosis but not significantly for LC. Age at diagnosis was strongly associated with latency length for both LC and MM ( $p<$ 0.001). Smoking, sex, cumulative exposure (log $\mathrm{f} / \mathrm{mL}$-year) and status at Wittenoom were not related to latency. Latency for LC with increasing age at first exposure declined faster than for MM.

Conclusions Age at diagnosis is associated with reduced shorter latency of MM and LC. Duration of exposure is associated with shorter latency of MM. Supported by NHMRC Australia.

Conflict of Interest No.

Journal Compilation (C) 2011 Asian Pacific Society of Respirology

\section{HEDGEHOG PATHWAY IN MALIGNANT MESOTHELIOMA}

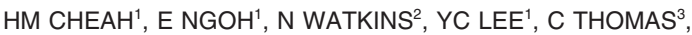
S MUTSAERS

${ }^{1}$ Lung Institute of WA, ${ }^{3}$ PathWest Laboratory of Medicine, ${ }^{2}$ Monash University, Vic.

Malignant mesothelioma (MM) is an aggressive cancer which is often associated with exposure to asbestos and SV40. This disease has a high latency period and a low survival rate. Therefore, new strategies for therapeutic intervention must be developed. Recent studies have shown that developmental pathways including the Hedgehog $(\mathrm{HH})$ pathway are associated with various types of cancers. The aberrant activation of key Hedgehog pathway proteins has been shown to contribute to cancer progression. However, the role of this pathway in $\mathrm{MM}$ has yet to be explored. We hypothesize that aberrant activation of the $\mathrm{HH}$ pathway is a contributing factor for the development of MM. The mRNA expression of $\mathrm{HH}$ pathway genes; Sonic Hedgehog $(\mathrm{SHH})$, Patched -1 (PTCH-1), Smoothened (SMO) and GLI-1 were examined in MM cell lines and tumour tissues by RT-PCR and qRT-PCR. HH pathway proteins and mRNA expression and distribution were then observed in the tumours by immunochistochemistry and in situ hybridization. We used real-time superarrays to examine the change in expression of a panel of key $\mathrm{HH}$ pathway genes by activating and inhibiting the pathway. We showed that the key $\mathrm{HH}$ pathway genes are expressed in both the cell lines and tissue samples. Upon stimulation with the ligand $\mathrm{SHH}$, there was an increase in expression of Indian Hedgehog $(\mathrm{IHH})$ and $\mathrm{SHH}$ in most of the mouse and human cell lines that we looked at. Interestingly, for the transcription factor GLI-2, there was a significant decrease in both mouse and human cell lines. Inhibiting this pathway increased the expression of PTCH in the mouse and human cell lines. The expression and up-regulation of key $\mathrm{HH}$ pathway components in $\mathrm{MM}$ at baseline and following stimulation suggests a role for the pathway in MM. Supported by NH\&MRC. Conflict of Interest No.

\section{AN AGGRESSIVE SURGICAL POLICY CAN BE ASSOCIATED WITH GOOD OUTCOMES IN STAGE 1 NON-SMALL CELL LUNG CANCER}

\section{Y DJAVADKHANI, P FLYNN, MJ PETERS, L MORGAN,} NEPEAN MULTIDISCIPLINARY LUNG CANCER GROUP Nepean Hospital, Kingswood, NSW

Aim To assess overall survival of patients following resection for Stage 1 NSCLC at a centre that has substantially greater resection rates than the NSW average.

Methods A retrospective audit of those patients who underwent lung resection for Stage 1 NSCLC at Nepean Hospital between January 2005 and February 2008.

Results 57 patients (33M:24F), mean age 68 (range 51-85) underwent resection. There were 2 pneumonectomies, 2 bilobectomies and 9 segmentectomies, one involving chest wall resection. The remaining procedures were lobectomies. There was one perioperative death from respiratory failure. Actuarial overall survival at 12 months was $84 \%$, at 24 months, $76 \%$ and at 5 years $47 \%$. Survival was not influenced by histology or age.

Conclusion In our institution, we have an agreed aggressive approach to resection of Stage $1 \mathrm{NSCLC}$ and our resection rate is $87 \%$. This pro-surgical policy is associated with good perioperative and long-term overall survival. Conflict of Interest No. 
TP-104

\section{INDWELLING PLEURAL CATHETERS SIGNIFICANTLY REDUCED HOSPITAL ADMISSIONS IN PATIENTS WITH MALIGNANT PLEURAL EFFUSIONS}

E FYSH $^{1,2}$, E GEELHOED ${ }^{1}$, P KENDALL $^{3}$, P BREMNER $^{3}$, G WATERER ${ }^{4}$, $\mathrm{J}^{2}$ LEONG $^{4}$, M MILLWARD $^{1}$, AW MUSK ${ }^{1,5}$, S MOREY ${ }^{5}$, G LEE $^{1,2}$

${ }^{1}$ University of Western Australia, ${ }^{2}$ Lung Institute of Western Australia, ${ }^{3}$ Fremantle Hospital, ${ }^{4}$ Royal Perth Hospital, and ${ }^{5}$ Sir Charles Gairdner Hospital, Perth, Australia

Introduction Malignant pleural effusions (MPEs) are common, although their management varies widely. Providing ambulatory care to minimize hospitalization is a key goal for patients with MPEs. Indwelling pleural catheters (IPCs) are a new treatment strategy that allows outpatient fluid drainage. We hypothesized that MPE patients managed with IPCs require fewer hospital admissions.

Methods A prospective, multicentre, non-randomized study involving all three major respiratory centres in Western Australia. Patients diagnosed to have MPEs were prospectively followed, and admissions were recorded. In the absence of accepted guidelines for IPC use, the choice of treatments (thoracentesis, IPC, pleurodesis) was decided by clinicians in-charge. All complications were recorded. Bacterial cultures of pleural fluid were performed monthly for patients with IPCs.

Results Of 54 patients with MPE, 21 were managed with IPCs and 33 by other methods (10 talc pleurodesis and 23 repeated thoracentesis). Mean follow-up was 73 days. IPC-treated patients had significantly fewer days in hospital (median, 25-75th percentiles) at $4.0(2.5-17.0)$ days over those who received other treatments at 10.0 days $(5.5-18.5), p=0.045$. Admission times solely for MPE-related causes were also significantly lower: 2.0 (1.0-6.0) versus $10.0(4.0-15.5)$ days, $p<0.001$. Four IPCs became colonized with coagulase-negative Staphylococci. No patients developed empyema.

Conclusion IPCs significantly reduce hospital admissions in MPE patients. Funded by State Health Research Advisory Council (Department of Health, Western Australia).

Conflict of Interest No.

\section{TP-105}

COMPARISON OF HISTOLOGICAL SUBTYPING BETWEEN DIAGNOSTIC SAMPLES AND RESECTED NON-SMALL CELL LUNG CANCER (NSCLC)

HM GALLAGHER ${ }^{1}$, EE DUHIG ${ }^{2}$, IA YANG ${ }^{1}$, RV BOWMAN ${ }^{1}$, BE CLARK $^{2}$, HM MARSHALL', KM FONG

'Thoracic Medicine, The Prince Charles Hospital and The University of Queensland, and ${ }^{2}$ Anatomical Pathology, The Prince Charles Hospital, Qld

Aim To determine the concordance of histological subtyping of NSCLC in diagnostic samples to the gold-standard lung resection specimens.

Methods We have so far evaluated 301 consecutive subjects who underwent curative surgery for primary NSCLC at The Prince Charles Hospital between the years 2006 and 2010. Many of these had workup at other institutions. One hundred forty-seven had Queensland health electronic record of positive preoperative diagnostic sampling. We correlated the final NSCLC WHO histological subtype with the subtypes diagnosed by samples prior to surgery including sputum, fiberoptic bronchoscopy (FOB) and trans-thoracic needle aspiration (TTNA). The resection subtype was set as the reference standard, and concordance was compared.

Results Of the 301 cases of resected NSCLC, 147 had malignancy on diagnostic sampling pre-resection, as shown in the Table:

\begin{tabular}{lcc}
\hline Resected Subtype & FOB concordant/FOB + ve & TTNA concordant/TTNA + ve \\
\hline Adenocarcinoma & $17 / 29(58 \%)$ & $23 / 39(59 \%)$ \\
Squamous cell & $21 / 27(78 \%)$ & $9 / 19(47 \%)$ \\
carcinoma (SCC) & & \\
Other & $6 / 19(32 \%)$ & $2 / 14(14 \%)$ \\
\hline
\end{tabular}

Discussion Of resected NSCLC with positive pre-resection diagnostic samples, the concordance between cytological subset classification of diagnostic samples to resection samples was moderate. Concordance was higher for SCC bronchoscopy samples. There was no difference in concordance between FOB and TTNA in this limited sample size; a larger cohort is being examined. NSCLC subset mutation testing and treatment decisions based on small diagnostic samples may result in potential misclassification of NSCLC subtype.

Supported by NHMRC Practitioner Fellowship (KF).

Conflict of Interest No.
POSTER WITHDRAWN 
THE PATTERN OF CARE FOR MALIGNANT PLEURAL MESOTHELIOMA (MPM) PATIENTS COMPENSATED BY THE NSW DUST DISEASES BOARD

\author{
S KAO ${ }^{1,2,3}$, N VAN ZANDWIJKK $^{1,3}$, S CLARKE ${ }^{2,3,4}$, J VARDY ${ }^{2,3}$, P CORTE $^{5}$, \\ C CLARKE ${ }^{1}$ \\ ${ }^{1}$ Asbestos Diseases Research Institute, NSW, ${ }^{2}$ Department of Medical \\ Oncology, Sydney Cancer Centre, NSW, ${ }^{3}$ University of Sydney, NSW, \\ Australia, ${ }^{4}$ Department of Medical Oncology, Royal North Shore Hospital, \\ NSW, and ${ }^{5}$ Department of Respiratory Medicine, Royal Prince Alfred \\ Hospital, NSW
}

Aim To determine the pattern of diagnosis and outcome of MPM patients and to assess the geographical variation of these patients in Australia.

Methods Consecutive consenting applicants for compensation at the Dust Diseases Board (DDB) from March 07 to 09 were included. Information were collected from the DDB and where necessary, the treating physicians and hospitals. Overall survival (OS) from diagnosis was determined by the Kaplan Meier method.

Results 159 patients were included: median age 71 years (range 45-91); $93 \%$ male; $65 \%$ living in major cities versus $35 \%$ in regional areas; $62 \%$ rightsided MPM; $60 \%$ epithelial subtype. Median time from asbestos exposure to diagnosis was 51 years (range 11-72). Median time from first symptoms or investigations to diagnosis was 6 weeks (range $0-140$ ). All patients had at least one chest X-ray and CT scan and $18 \%$ had PET scan. A variety of procedures led to the diagnosis: $75 \%$ thoracoscopy, $15 \%$ thoracotomy, $4 \%$ radiology-guided, $2 \%$ chest wall biopsy and $2 \%$ medical pleuroscopy, with $4 \%$ having had cytology alone. Median number of diagnostic immunohistochemical stains used was 7 (range $0-18$ ), with calretinin (94\%) the most commonly used mesothelial marker and carcinoembryonic antigen (CEA; 72\%) the most common carcinoma marker. Median OS for the cohort was 11.7 months $(95 \%$ $\mathrm{Cl}$ : 9.1-14.2), with no statistical difference in OS between major city and regional patients ( 11 vs. 12.5 months, respectively, $p=0.35$ ).

Conclusions MPM appeared to affect mainly the elderly, and thoracoscopy was the most common diagnostic procedure. OS did not differ between Australian major city and regional patients and was comparable to the largest phase III trial in MPM.

Supported by Dust Diseases Board Research Grant.

Conflict of Interest No.

TP-109

MURINE LUNG TUMOUR DEVELOPMENT IS EXACERBATED BY DEREGULATED GP130 SIGNALLING

A MILLER, S RUWANPURA, L MCLEOD, P BARDIN, N WATKINS, BJ JENKINS

Monash Institute of Medical Research, Monash University, Melbourne, Victoria

Rationale Lung cancer is the leading cause of cancer death worldwide. It is well established that cigarette smoking is linked to emphysema and lung cancer, and smokers with emphysema are at an increased risk of developing lung cancer. Notably, recent epidemiological studies have indicated that emphysema can predispose to lung cancer irrespective of pack-year smoking history. Although inflammation has been proposed as a common mechanism linking these two diametrically opposed diseases, the conceptual inter-relationship between inflammation, emphysema and lung cancer has been poorly investigated because existing experimentally induced and genetically modified animal models for lung cancer occur in the absence of emphysema.

Method We have utilized a newly identified mouse model (gp130 $1 / \mathrm{F})$ of spontaneous lung inflammation and emphysema in two well-established lung cancer models. The gp $130^{\mathrm{F} / \mathrm{F}}$ mouse is characterized by deregulated cytokine signalling via gp130, the critical co-receptor for the interleukin (IL)-6 cytokine family, leading to hyper-activation of STAT3, a potent pro-inflammatory and oncogenic latent transcription factor. In 2 separate studies, we exposed $\mathrm{gp} 130^{\mathrm{FF}}$ mice to a cigarette-derived carcinogen (NNK), and crossed them with the genetically susceptible KRas(G12D) strain of mice.

Results In both NNK- and KRas(G12D)-induced lung cancer models, the lungs of $\mathrm{gp} 130^{\mathrm{F} / \mathrm{F}}$ mice were highly predisposed to hyperplasia and tumour formation. Increased levels of cellular proliferation were observed in hyperplastic and tumour lesions, as well as surrounding areas, of these mice. These observations were verified at the molecular level by gene expression profiling of tumour-bearing lung tissue.

Conclusions These studies provide unique insights into the importance of interactions between the gp130 signalling axis and factors that predispose to lung tumourigenesis in emphysema.

Support NHMRC

Conflict of Interest No.

Journal Compilation (C) 2011 Asian Pacific Society of Respirology

\section{INCIDENCE RATES OF LUNG CANCER (LC) AND MALIGNANT MESOTHELIOMA (MM) IN THE WITTENOOM COHORTS 50 YEARS AFTER LAST EXPOSURE TO CROCIDOLITE}

AW MUSK ${ }^{1,2}$, P ABOAGYE-SCARFO ${ }^{1}$, A REID $^{1,4}$, N OLSEN ${ }^{1}$, L SAMUELS ${ }^{1}$, P FRANKLIN1, N DE KLERK ${ }^{1,3}$

${ }^{1}$ School of Population Health, UWA, Crawley, WA, ${ }^{2}$ Sir Charles Gairdner Hospital, Nedlands, WA, ${ }^{3}$ Centre for Medical Research, UWA, Crawley, WA, and

${ }^{4}$ Telethon Institute of Child Health Research, Subiaco, WA

Aim To estimate changes in incidence rates of LC and MM between 1943 and 2006.

Background Crocidolite (blue asbestos) was mined and milled at Wittenoom West Australia from 1943 to 1966.6500 men and 500 women were employed and 5000 people lived in the town to December 2006 (not employed). All have been followed for cancer incidence and deaths to December 2006.

Subjects Workers and residents cohorts were established from employment records, electoral rolls, medical fund contributions, school and medical attendance records between 1953 and May 2010. Five hundred eight LCs and 439 MMs with exposure estimates were included.

Methods Incident cases of LC and MM were identified from the Australian and West Australian Cancer, Mesothelioma, and Death Registries. Rates were calculated for 5-year intervals. Exposures to crocidolite (fibre/mL.yrs) for all members of the cohorts were based on measures of dustiness in the industry and the town for the periods of employment and residence. Smoking rates have been obtained by periodical questionnaires.

Results Average time since first exposure was 50 years. The incidence rates of LC and MM per 1000 person-years have continued to increase from 19 $(95 \% \mathrm{Cl}=13,29)$ and 0 in 1966 to $26(95 \% \mathrm{Cl}=22,30)$ and $28(95 \% \mathrm{Cl}=$ 24,33 ) in 2006. Smoking was a significant risk factor for LC with current smokers at higher risk $(\mathrm{HR}=14.86,95 \% \mathrm{Cl}=7.57,29.66)$, but there was no significant association between smoking and MM. Every unit increase of log (fibre/mL.yrs) increased the rate of LC and MM. After adjusting for time since first exposure, age and sex, the increase in rate of MM was significant $(p<$ 0.001 ) but the increase in rate of LC was not significant.

Conclusion LC and MM rates remain excessive and dose-related. MM rates continue to rise in these cohorts 50 years since first exposure to crocidolite. Supported by NHMRC Australia.

Conflict of Interest No.

\section{TP-111}

SOCS6: A POTENTIAL TUMOUR SUPPRESSOR GENE INVOLVED IN THE RECURRENCE OF LUNG SQUAMOUS CELL CARCINOMA

KB SRIRAM ${ }^{1,2}$ JE LARSEN ${ }^{2}$, SM SAVARIMUTHU FRANCIS ${ }^{1,2}$, CM WRIGHT' ${ }^{1}$, BE CLARKE ${ }^{1}$, EE DUHIG ${ }^{1}$, NK HAYWARD ${ }^{3}$, RV BOWMAN 1,2, IA YANG ${ }^{1,2}$, KM FONG $^{1,2}$

${ }^{1}$ The Prince Charles Hospital, ${ }^{2}$ University of Queensland, and ${ }^{3}$ Queensland Institute of Medical Research, Brisbane, Qld

Genes with aberrant DNA copy number may be involved in the early recurrence of surgically resected primary lung squamous cell carcinoma (SCC) Aim To use array comparative genomic hybridization (aCGH) and identify recurrence SCC-specific genes that have DNA copy number aberrations.

Methods aCGH was performed using Agilent CGH 44B (Agilent Technologies Inc) microarrays on DNA from fresh frozen tumour tissue obtained from 33 non-recurrence SCC subjects (no recurrence of disease at 36 months) and 25 recurrence SCC subjects (recurrence of disease within 18 months). aCGH data were analysed using the Genomic Identification of Significant Targets in Cancer (GISTIC) algorithm. Validation of the DNA copy number of candidate genes was performed using quantitative PCR (q-PCR).

Results GISTIC identified that deletions in 18q22.3 occurred more frequently in recurrence SCCs compared to non-recurrence SCCs $(52 \%$ vs. $24 \%$, p < 0.001). Within 18q22.3, 7 genes, SOCS6, DOK6, RTTN, CYB5A, C18orf55, NETO1 and CCDC102B, had lower DNA copy number in recurrence SCCs ( $\mathrm{p}$ $<0.05$ for all 7 genes). q-PCR of the candidate genes in the training cohort, demonstrated that only SOCS6 as having a lower DNA copy number in recurrence SCCs compared to non-recurrence SCCs $(p=0.003)$. Furthermore, in an independent cohort consisting of 22 non-recurrence SCCs and 9 recurrence SCCs, SOCS6 had a lower DNA copy number in the recurrence SCC samples $(\mathrm{p}=0.006)$.

Conclusion SOCS6 appears to be a putative tumour suppressor gene that is deleted particularly in recurrence SCCs.

Supported by NHMRC Medical Postgraduate Scholarship.

Nomination Janet Elder International Travel Award.

Conflict of Interest No. 
OELD/POPULATION HEALTH SIG: POSTER SESSION

TP-112

PREPAREDNESS OF HEALTH-CARE FACILITIES FOR AN INFLUENZA PANDEMIC - PROTECTING THE HEALTH-CARE WORKERS

AL HOLLINGWORTH

Respiratory Medicine, St Vincent's Hospital, NSW 2010, Australia

Aim To assess the preparedness of hospitals with respect to protecting health-care workers (HCWs) during a pandemic.

Methods A self-administered questionnaire was performed between November 2009 and January 2010, and a scoring system was developed to provide a quantifiable measure of preparedness.

Results A total of 12 hospitals in NSW, Australia, were approached - six regional hospitals (RHs) and six tertiary referral centres (TRCs). The study was extended to assess three hospitals in England, allowing a limited comparison between the hospitals in Australia that had faced the initial wave of the H1N1 ('swine flu') pandemic and the hospitals in the UK that had more time to prepare for the outbreak. Response rates were $66 \%$ from the TRCs, $33 \%$ from the $\mathrm{RHs}$ and $100 \%$ from the English hospitals. The overall preparedness scores were relatively high, with a median total score (adjusted) of 50.75 out of 70 . The demographic that scored the highest Total was tertiary referral centres in Sydney. All English hospitals scored below the median. However, the range of scores across hospitals was quite narrow (45.1-57.1 adjusted). Scores were generally high for the areas of Preparedness, Infection control, Education and Training. Scores for Vaccination were more variable. The category that consistently demonstrated the lowest scores was that of Psychosocial Welfare and Assistance, despite this found in previous research to be an integral part of that which HCWs have identified as important.

Conclusions Given their integral role in pandemic response, protecting HCWs must be a priority as part of any pandemic preparedness plan. This goes beyond protection from infection, extending into aspects of physical and psychological wellbeing. Identifying these issues and addressing them is the key to maximizing staff support and morale, and minimizing staff absenteeism at such a crucial time.

Conflict of Interest No.

THE ASSOCIATION BETWEEN REFLUX AND RESPIRATORY SYMPTOMS IS INDEPENDENT OF BODY MASS INDEX AND OBSTRUCTIVE SLEEP APNOEA IN BUSSELTON ADULTS

SA MULRENNAN ${ }^{1}$, MW KNUIMAN², ML DIVITINI², DJ CULLEN ${ }^{3}$, M HUNTER ${ }^{3}, \mathrm{~J} \mathrm{HUI}^{3}$, AW MUSK ${ }^{1,3}$, AL JAMES $^{3,4}$

${ }^{1}$ Dept of Respiratory Medicine and ${ }^{3}$ Busselton Population Medical Research Foundation, ${ }^{2}$ School of Population Health, University of Western Australia, Crawley, WA, and ${ }^{4}$ Dept of Pulmonary Physiology/West Australian Sleep Disorders Research Institute, Sir Charles Gairdner Hospital

Aim To describe the relationship of respiratory and reflux symptoms within the general population and relate this to the possible confounding factors of body mass index (BMI) and obstructive sleep apnoea (OSA).

Methods Data from a cross-sectional health survey, performed in Bussleton, West Australia in 2005-2007, were used to examine the relative effects of BMI and OSA on the relationship between respiratory and reflux symptoms. Questionnaire data included information on asthma, cough, wheeze, dyspnoea and GORD symptoms. GORD symptoms were categorized as never, monthly or less often and weekly or more often. BMI, risk of OSA defined according to the Berlin questionnaire, spirometry and airway hyperresponsiveness to methacholine were also recorded. Logistic regression models obtained odds ratios for the associations between each GORD symptoms, various respiratory symptoms, BMI and OSA.

Results Average age was 55 years and recent wheeze was reported in $23 \%$ and cough and phlegm in $23 \%$. Twelve percent were current smokers. AHR was present in $12 \%$ and OSA in $23 \%$. GORD symptoms occured in $50 \%$ and frequent symptoms (weekly or more often) were present in $5-10 \%$. There were strong positive associations between GORD symptoms and cough/phlegm, breathlessness, chest tightness and wheeze in the last 12 months. Odds ratios increased with increasing frequency of reflux $p \leq 0.001$. There was no effect of obesity or OSA on the relationship between respiratory and GORD.

Conclusion Cough and phlegm, breathlessness, chest tightness and wheeze (ever or recent) are all strongly associated with symptoms of GORD. This relationship is amplified with increasing frequency of GORD symptoms indicating a dose-response relationship between reflux and respiratory symptoms. Obesity and OSA do not affect the association between GORD and respiratory symptoms.

Supported by NH\&MRC grant (ID: 353532).

Conflicts of Interest Nil.
ACUTE EXPOSURE TO DIESEL EXHAUST PARTICLES ELICITS DOSE-DEPENDENT PULMONARY INFLAMMATION AND IMPAIRMENT OF LUNG FUNCTION IN NAÏVE BALB/C MICE

AN LARCOMBE ${ }^{1,2}$, CE BOYLEN ${ }^{1,2,3}$, PD SLY ${ }^{4}$, GR ZOSKY $Y^{1,2}$

${ }^{1}$ Telethon Institute for Child Health Research, ${ }^{2}$ Centre for Child Health Research, University of Western Australia, Perth, WA, ${ }^{3}$ Murdoch University, Perth, WA, and ${ }^{4}$ Queensland Children's Medical Research Institute, University of Queensland, Brisbane, Qld

Aim Diesel exhaust particles (DEP) produced from the combustion of diesel fuel are the major contributor to particulate pollution in urban areas. Previous studies show significant impacts on lung function as a result of chronic DEP exposure; however, the effects of a single acute exposure in a healthy lung are unknown. This study aimed to examine the effects of a single instillation of DEP on pulmonary inflammation and lung function and to determine the role sex and dose play in these responses.

Methods Mice were intranasally inoculated with 10,30 or $100 \mu \mathrm{g}$ DEP. At predetermined time points, mice were euthanized and bronchoalveolar lavage taken for analysis of inflammatory cells, cytokines and phagocytosis of DEP by alveolar macrophages. Lung function was measured 6 and 24 hours postinoculation with $100 \mu \mathrm{g}$ DEP using the forced oscillation technique and plethysmography.

Results The recruitment of inflammatory cells (macrophages and neutrophils) was dose dependent and similar between male and female mice with the greatest response being measured in mice inoculated with $100 \mu \mathrm{g}$ DEP. A single dose of $100 \mu \mathrm{g}$ DEP was sufficient to induce impairments in lung function at FRC, and the volume dependence of lung mechanics in female, but not male, mice.

Conclusions We have shown that acute exposure to DEP elicits dose- and sex-dependent pulmonary inflammation and impairment of lung function in naïve BALB/c mice.

Supported by NH\&MRC, Murdoch University.

Conflict of Interest No.

\section{ACUTE DIESEL EXHAUST PARTICLE EXPOSURE INCREASES VIRAL TITRE ASSOCIATED WITH INFLUENZA BUT DOES NOT EXACERBATE INFLAMMATION OR DEFICITS IN LUNG FUNCTION}

RE FOONG ${ }^{1,2}$, CE BOYLEN ${ }^{1,2}$, AN LARCOMBE ${ }^{1,2}$, GR ZOSKY $Y^{1,2}$

${ }^{1}$ Telethon Institute for Child Health Research, and ${ }^{2}$ Centre for Child Health Research, University of Western Australia, WA, Australia

Introduction Diesel exhaust particles (DEP) make up the bulk of particulate matter in urban areas. High ambient levels of particulate matter are associated with increased hospitalization due to respiratory disease. We aimed to determine if exposure to DEP exacerbates responses to acute viral infection.

Methods Adult female BALB/c mice were inoculated with $100 \mu \mathrm{g}$ DEP or control 3.75 days after infection with $10^{4.5}$ plaque forming units (pfu) of influenza A/Mem71 (or control). Six hours after DEP inoculation, lung volume (TGV) and lung mechanics were measured by plethysmography and the forced oscillation technique, respectively. Bronchoalveolar lavage fluid was collected to assess cellular inflammation and cytokine levels.

Results Viral titre was significantly higher in influenza-infected mice exposed to DEP compared to those exposed to influenza alone $(p=0.04)$. Both DEP $(p=0.03)$ and influenza infection $(p<0.001)$ alone significantly increased cellular inflammation; however, there was no difference between mice exposed to both DEP and influenza compared to those exposed to influenza alone ( $p$ $=0.42$ ). A similar pattern was found in levels of cytokines in the bronchoalveolar lavage (TNF- $\alpha$, MCP-1, IL-6, IFN- $\gamma$ ). Specific airway resistance, specific tissue damping, specific tissue elastance and hysteresivity were significantly increased in influenza infected mice ( $p<0.001$ in all cases). None of these parameters were influenced by DEP exposure alone ( $p>0.33$ in all cases) and there was no additive effect of DEP on lung function ( $p>0.22$ in all cases) in influenza-infected mice.

Conclusions DEP increases viral titre but is not sufficient to physiologically exacerbate pre-existing respiratory disease caused by influenza infection in mice.

Supported by NHMRC.

Conflict of Interest No. 
THE RISK OF AIRBORNE INFLUENZA TRANSMISSION IN PASSENGER CARS

LD KNIBBS', L MORAWSKA', SC BELL ${ }^{2}$

ILAQH, ${ }^{1}$ QUT, Brisbane, and ${ }^{2}$ Thoracic Medicine, The Prince Charles

Hospital, Brisbane, Australia

Influenza is a common cause of mortality, morbidity and loss of productivity Aerosol transmission is likely to contribute to human-to-human spread (e.g. houses, schools, aircraft).

Aim The risk of transmission in car travel is unknown, and we performed a measurement- and model-based investigation to address this.

Methods Air exchange rate measurements were performed using sulphur hexafluoride $\left(\mathrm{SF}_{6}\right)$. Three distinct ventilation settings were assessed in two hatchbacks with windows closed. Triplicate measurements were performed when the cars were stationary and at 60 and $110 \mathrm{~km} / \mathrm{h}$. Gammaitoni and Nucci's equations were used to model to predict infection risk. We used an infectious quanta generation rate of 67 quanta per hour in our simulations. Infection risk was calculated for each combination of ventilation setting, speed and trip time.

Results Outdoor air exchange showed large variability related to car age and design. During high ventilation setting, air exchange was similar to having a window partly opened. Under the low ventilation condition when air was recirculated, infection risks in Car A ranged from 31 to $68 \%$ for a $90-$ minute trip at speeds ranging from 25 to $100 \mathrm{~km} / \mathrm{h}$. In Car B, risks were approximately $99 \%$ in all cases. Under the medium and high ventilation settings, risk at 90 minutes ranged from 20 to $35 \%$ and 16 to $21 \%$.

Conclusions Transmission risks in cars were strongly influenced by vehicle characteristics and ventilation. Risk reductions can be achieved by not recirculating air.

Supported by NHMRC, ARC

Nomination Nil.

Conflict of Interest Nil.

\section{OLIV SIG: POSTER SESSION}

\section{PULMONARY HYPERTENSION PREDICTS HYPOXAEMIA DURING AIR TRAVEL IN PATIENTS WITH INTERSTITIAL LUNG DISEASE}

TJ CORTE ${ }^{1,2}$, G KEIR $^{1}$, D CRAMER ${ }^{1}$, S WARD ${ }^{1}$, C NELSON $^{1}$, D SHRIKRISHNAPALASURIYAR ${ }^{1}$, SJ WORT ${ }^{1}$, AU WELLS ${ }^{1}$

${ }^{1}$ Royal Brompton Hospital, London, UK, and ${ }^{2}$ Royal Prince Alfred Hospital, Sydney, Australia

Background In interstitial lung disease (ILD), neither pulmonary function nor $\mathrm{SpO}_{2}$ at sea level accurately predict hypoxaemia during air travel. We hypothesize that pulmonary hypertension $(\mathrm{PH})$ may contribute to hypoxaemia during air travel.

Methods All ILD patients undergoing hypoxic challenge testing (HCT) prior to air travel (January 2001-June 2010) were identified $(n=272)$. We studied 133 patients with concurrent pulmonary function testing and echocardiography. Patients breathed gas mixtures $\left(15 \% \mathrm{O}_{2}\right.$ in nitrogen) for 20 minutes or until equilibration. $\mathrm{SpO}_{2}$ was monitored and arterial blood gases taken before and after testing. Hypoxic response was defined as $\mathrm{PaO}_{2}<6.6 \mathrm{kPa}$ or $\mathrm{SpO}_{2}$ $<85 \%$. The relationship between hypoxic response to $\mathrm{HCT}$ and $\mathrm{PH}$ was explored. PH was considered present at echocardiography when systolic pulmonary arterial pressure $\geq 40 \mathrm{mmHg}$.

Results Mean FVC was $71 \pm 22 \%$; mean DLco was $42 \pm 14 \%$; mean $\mathrm{PaO}_{2}$ $10.5 \pm 1.2 \mathrm{kPa}$, and $32(24 \%)$ patients had $\mathrm{PH}$ on echocardiography. Following $\mathrm{HCT}$, the mean $\mathrm{PaO}_{2}$ was $6.8 \pm 0.8 \mathrm{kPa}$, and $60(45 \%)$ patients had a hypoxic response to HCT. Hypoxic response to HCT was independently associated with $\mathrm{PH}(\mathrm{OR} 2.5 ; \mathrm{Cl} 1.00,6.43 ; \mathrm{p}<0.05)$ and functional impairment (composite physiologic index, CPI: OR 1.07: $\mathrm{Cl} 1.04,1.10 ; \mathrm{p}<0.0001$ ) following adjustment for age and gender. The end-test $\mathrm{SpO}_{2}$ correlated with both $\mathrm{CPI}(\mathrm{R}=$ $-0.48 ; p<0.00001)$ and right ventricular systolic pressure at echocardiography $(\mathrm{R}=-0.28 ; \mathrm{p}=0.01)$.

Conclusion Hypoxic response to HCT is associated with PH and independent of the underlying ILD severity.

Conflict of Interest None.
ROOM VENTILATION AND THE RISK OF AIRBORNE INFECTION TRANSMISSION IN A TERTIARY HOSPITAL

LD KNIBBS ${ }^{1}$, L MORAWSKA ${ }^{1}$, P GRZYBOWSKI ${ }^{1,2}$, S BELL $^{3}$ ILAQH, ${ }^{1}$ QUT, Brisbane, ${ }^{2}$ Faculty of Chemical and Process Engineering, Warsaw University of Technology, Poland, and ${ }^{3}$ Thoracic Medicine, The Prince Charles Hospita ${ }^{3}$, Brisbane, Australia

Outdoor air ventilation is a key mechanism controlling the airborne spread of several diseases. However, ventilation guidelines for hospitals are not typically based on preventing infection transmission.

Aim We sought to assess the effectiveness of current ventilation rates on infection risks for influenza, tuberculosis (TB) and rhinovirus within three distinct rooms in a major tertiary hospital in Australia.

Methods The rooms targeted were a Lung Function Laboratory, negative pressure isolation room in the Emergency Department and an Outpatient Consultation Room. Air exchange measurements were performed in each by using $\mathrm{CO}_{2}$ decay, and the proportion of outdoor air supplied was determined by $\mathrm{CO}_{2}$ mass-balance at the air handling unit. Gammaitoni and Nucci's infection risk model, based on the traditional Wells-Riley model, was then employed to model scenarios typical of those experienced by patients.

Results Current outdoor air exchange rates in the Lung Function Laboratory and Isolation Room were appropriate, and infection risks for all modelled scenarios were $<3.6 \%$. Influenza risk for patients entering the OPD room after an infectious patient departed ranged from 3.6 to $20.7 \%$ depending on the occupancy time of the susceptible and infectious patient.

Conclusions In the absence of definitive guidelines, air exchange measurements combined with modelling afford a useful means of assessing, on a case-by-case basis, the suitability of room ventilation at preventing airborne transmission.

Supported by NHMRC.

Nomination Nil.

Conflict of Interest Nil.

\section{TP-119}

PREVENTING EPITHELIAL-MESENCHYMAL TRANSITION IN AIRWAY EPITHELIAL CELLS FROM LUNG TRANSPLANT PATIENTS

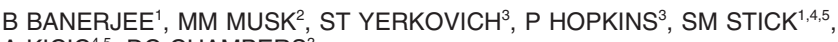
A KICIC $^{4,5}$, DC CHAMBERS ${ }^{3}$

${ }^{1}$ University of WA, WA, ${ }^{2}$ WA Lung Transplant Unit, RPH, WA, ${ }^{3}$ Queensland Centre for Pulmonary Transplantation \& Vascular Disease, QId, ${ }^{4}$ TICHR, WA, ${ }^{5}$ Department of Respiratory Medicine, PMH, WA

Introduction Lack of treatments for post-transplant obliterative bronchiolitis (OB) is mainly due to the poor understanding of its pathogenesis and lack of small airway models. Epithelial-mesenchymal transition (EMT) may play a central role and could be crucial to developing treatment drugs. We hypothesize that EMT induction may be prevented by pharmacologically available compounds.

Methods Primary cultures of small and large airway epithelial cells (SAEC and LAEC) were established and EMT induced by adding TGF $\beta 1(50 \mathrm{ng} / \mathrm{mL})$ $(\mathrm{n}=6)$. Azithromycin $(1-100 \mu \mathrm{M})$, mycophenolate $(0.05-5 \mathrm{mg} / \mathrm{L})$ and RAD001 $(0.01-1 \mathrm{ng} / \mathrm{L})$ were then added and expression of epithelial (ZO-1, Ck-19) and mesenchymal markers (Eda-Fn, Vim) measured via Western blot as well as MMP 2 and 9 activity via zymography.

Results Significantly lower increase in mesenchymal markers and lower decrease in epithelial markers, compared to controls was noted for azithromycin and mycophenolate indicating suppression of EMT. MMP 2 and 9 activity increase was also significantly suppressed. Azithromycin suppressed EMT to a greater extent compared to mycophenolate, but was equally effective in both small and large airway epithelia. RAD001 appeared to have no effect.

Conclusions Azithromycin and mycophenolate are both effective in preventing EMT and thus have potential for the clinical treatment of $\mathrm{OB}$.

Supported by ABN Foundation.

Conflict of Interest None. 
DIFFERENTIAL EXPRESSION OF PRO-INFLAMMATORY CYTOKINES BY INTRAEPITHELIAL T CELLS FROM GRAFT, TRACHEA OR NATIVE LUNG DISTINGUISHES SEVERITY OF ACUTE REJECTION

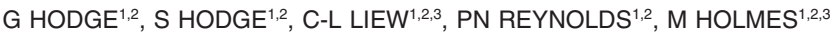
${ }^{1}$ Lung Research, Hanson Institute, ${ }^{2}$ Department of Thoracic Medicine, Royal Adelaide Hospital, and ${ }^{3}$ South Australian Lung Transplant Service, Adelaide

T-cell pro-inflammatory cytokines are associated with acute lung transplant rejection. We have previously shown compartmentalization of production of these cytokines in bronchial intraepithelial T cells (IET) obtained by bronchial brushings from stable lung transplant patients. During acute rejection episodes, no significant differences in IET cytokines were observed between stable and rejecting patients due to broad cytokine variability between patient groups. To overcome this limitation, we hypothesized that there would be increased graft pro-inflammatory IET cytokines compared with native lung or trachea during acute rejection.

Methods Cell cultures from stable patients, patients with evidence of acute rejection and BOS and healthy controls were stimulated and intracellular cytokines determined using multiparameter flow cytometry.

Results There was a significant increase in graft IET-cell IFN $\gamma$ and TNF $\alpha$ in the lungs of patients with acute rejection compared with IET cells obtained from the native lung or trachea, but no changes were noted between other patient groups. There was a significant correlation between increased graft IET-cell TNF $\alpha$ compared with trachea and lungs and acute rejection grade. Conclusions Differential expression of pro-inflammatory cytokines by IET cells from graft, trachea or native lung distinguishes severity of acute rejection. Improved monitoring response using this assay or therapeutic targeting of these pro-inflammatory cytokines may reduce acute lung transplant rejection.

Supported by NHMRC

Conflict of Interest Nil.

\section{PREVALENCE OF REDUCED CARBON MONOXIDE TRANSFER FACTOR IN THE ABSENCE OF AN IDENTIFIABLE CAUSE}

J WILES, J GOLDIN, B JENNINGS, D SMALLWOOD, L IRVING

Department of Respiratory and Sleep Medicine, Royal Melbourne Hospital, Parkville, Victoria, Australia

Aim To determine the prevalence of reduced carbon monoxide transfer factor (DLCO $\leq 70 \%$ predicted) in subjects undergoing pulmonary function testing (PFTs) and to determine whether a cause has been identified.

Methods A clinical audit of all subjects undergoing PFTs at Royal Melbourne Hospital from 1 August 2007 to 31 August 2009 who have a DLCO $\leq 70 \%$ in the setting of normal spirometry. Medical records and investigations including transthoracic echocardiogram (TTE), high-resolution commuted tomography (HRCT), ventilation/perfusion (V/Q) scans were reviewed to determine whether a cause for the reduced DLCO was established. Where a cause was not clear, subjects were invited to participate in a telephone interview to evaluate symptoms and to undergo repeat PFTs. Subjects with a persistently reduced DLCO were invited to undergo further investigation with TTE, HRCT and V/Q scan. Preliminary Results PFT results from 2412 subjects were reviewed. Subjects with $\mathrm{FEV}_{1} / \mathrm{FVC}<70, \mathrm{FEV}_{1}<80 \%$ predicted and $\mathrm{FVC}<80 \%$ predicted were excluded. Three hundred seventy subjects $(15 \%)$ had an isolated reduction in DLCO. 149/370 (40\%) of these subjects underwent TTE with 34/149 (23\%) demonstrating an elevated right ventricular systolic pressure (RVSP). In all cases where there was an elevated RVSP an identifiable cause was found. $8 / 34(24 \%)$ of these subjects subsequently identified as having Pulmonary Arterial Hypertension (PAH) and commenced appropriate therapy and $7 / 34(21 \%)$ identified as having $\mathrm{PAH}$ where treatment was not commenced. There were $221 / 370(60 \%)$ of subjects who appeared not to have undergone a TTE. Further evaluation of medical records of subjects who had not undergone TTE and those with normal TTE is continuing. Review of subjects HRCT, $V / Q$ scans and right heart catheterizations is currently proceeding.

Conclusions Preliminary results suggest that a significant proportion of subjects with isolated reduction of DLCO on PFTs do not undergo TTE which is an important investigation in determining the cause for the reduced DLCO. When a TTE is performed and demonstrates an elevated RVSP, a cause for the elevated RVSP is identified.

Sponsor Actelion Pharmaceuticals Australia Pty Ltd

Conflict of Interest No.

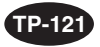

BOS IS ASSOCIATED WITH INCREASED PERIPHERAL BLOOD NKT-LIKE AND NK CELL GRANZYMES, PERFORIN AND TH1 PRO-INFLAMMATORY CYTOKINES

G HODGE ${ }^{1,2}$, S HODGE ${ }^{1,2}$, C-L LIEW'1,2,3, PN REYNOLDS ${ }^{1,2}$, M HOLMES $^{1,2,3}$ ${ }^{1}$ Lung Research, Hanson Institute, ${ }^{2}$ Department of Thoracic Medicine, Royal Adelaide Hospital, Adelaide, and ${ }^{3}$ South Australian Lung Transplant Service

Background T-cell pro-inflammatory mediators are associated with acute lung transplant rejection. We have previously shown that BOS was associated with lack of immunosuppression of T-cell pro-inflammatory cytokines and increased T-cell granzyme B in peripheral blood. Recently, we also showed that NKT-like cells are a major source of pro-inflammatory cytokines and granzymes in the blood of stable lung transplant patients. We hypothesized that BOS may be associated with lack of immunosuppression of these proinflammatory mediators in blood NK and NKT-like cells.

Method Granzyme/perforin profiles from stable patients, patients with evidence of BOS and healthy controls were determined and blood cultures stimulated and intracellular cytokines determined using multiparameter flow cytometry.

Results There was a significant increase in the percentage of NK cells expressing granzymes and perforin in BOS patients compared with stable patients and controls. There was an increase in the percentage of T, NK and NKT-like cells producing IFN $\gamma$ and TNF $\alpha$ in BOS compared with stable patients. There was a significant correlation between increased NK IFN $\gamma$ and TNF $\alpha$ and FEV

Conclusions BOS is associated with increased peripheral blood NKT-like and NK cell granzymes, perforin and Th1 pro-inflammatory cytokines. Therapeutic targeting of these pro-inflammatory mediators and monitoring response using this assay may reduce BOS.

Supported by NHMRC.

Conflict of Interest Nil.

\section{A CASE REVIEW STUDY OF INVESTIGATING FOR PULMONARY EMBOLISM IN PREGNANCY}

P HULLAH, B HARRIS, M HIBBERT

Respiratory Medicine, Royal North Shore Hospital, NSW, Australia

Rationale Pulmonary embolism (PE) is the leading cause of maternal mortality in the developed world. Consequently accurate diagnosis of PE is critical. This must be tempered by the potential radiation risk of investigations to the mother and foetus. We performed a retrospective case review to determine the incidence of PE in pregnant patients investigated for this condition. Demographic information, the diagnostic algorithm utilized and the diagnostic yield of investigations were obtained.

Method Pregnant women who underwent Ventilation Perfusion (VQ) scanning or Computed Tomography Pulmonary Angiogram (CTPA) at our institution between January 2005 and January 2010 were identified by an internal database audit. In addition to demographic data, information about the diagnostic pathway and final diagnosis were collected. In cases where PE was not diagnosed, the medical records were reviewed for any subsequent events up until the date of delivery.

Results During the five-year period, $28 \mathrm{VQ}$ scans and 20 CTPAs were performed on 46 pregnant women. The average gestation at investigation was 26 weeks. Only one patient had a previous history of venous thrombo-embolism. 50\% underwent Doppler ultrasound of the lower limbs prior to VQ or CTPA. Overall the incidence of PE was $2 \%$, diagnosed by VQ scan. Otherwise the VQ scans were normal in $46 \%$, low probability in $42 \%$ and non-diagnostic in $8 \%$ cases. CTPA was non-diagnostic in $2 \%$ of cases. All other CTPA studies demonstrated no emboli. Almost $50 \%$ of scans were done after hours $(50 \%$ VQ and $40 \%$ CTPA). No patients without PE were felt to have had the PE missed up to the time of delivery.

Conclusions The overall incidence of $\mathrm{PE}$ in patients being investigated was extremely low at $2 \%$. During this study period slightly more VQ studies were performed than CTPAs, with each test having similar diagnostic rates. Only $50 \%$ of patients had undergone venous Doppler prior to undergoing radiationexposing investigations.

Nomination Nil.

Conflict of Interest None.

Journal Compilation (c) 2011 Asian Pacific Society of Respirology 


\section{RO-52 ANTIBODIES AND IDIOPATHIC INTERSTITIAL PNEUMONIA}

\section{Z BHIKOO, D MILNE, M WILSHER}

Green Lane Respiratory Services, Auckland City Hospital, Auckland District Health Board, Auckland, New Zealand

Introduction Anti-Ro-52 antibodies have been associated with idiopathic interstitial pneumonia (IIP) in one small series $(n=4)$. We hypothesize that Ro-52 antibodies, just like myositis antibodies, can serve as a marker of undifferentiated connective tissue disease (CTD) with interstitial pneumonia as the primary phenotypic manifestation. The aim of this study was to examine the characteristics of patients with Ro-52 and IIP.

Methods Retrospective study identifying patients with IIP and Ro-52 positivity, but negative for CTD and/or myositis antibodies, presenting between June 2005 and June 2010. Data relating to demographics, diagnosis, pulmonary function tests, length of follow-up and outcome were obtained. All HRCT images were reviewed by an independent expert radiologist (DM).

Results 10/17 Ro-52 positive subjects fulfilled criteria ( 7 male, median age 64 (39-83), 8 European, 5 never smoked). 8/10 had Ro-52 titers above 40 and 2 in the intermediate (30-40) range. Three patients had Raynauds phenomenon; there were no other CTD features. 7/10 patients had HRCT diagnosis of NSIP and 3/10 organizing pneumonia; 7/10 had extensive fibrosis. Mean (SD) \% predicted baseline FVC 66(20), DLCO 46(16). Median length of follow-up was 29 months. All patients were treated and 8 were considered overall stable at last follow-up, one had declined and one died of respiratory failure.

Conclusion This study confirms an association between Ro-52 positivity and interstitial pneumonia in the absence of defined connective tissue disease, suggesting an autoimmune basis for the interstitial lung disease in this group of patients. A larger cohort is required to determine the true significance of this observation.

Conflict of Interest Nil.

\section{TP-125}

\section{IMPACT OF NEBULISED COLISTIN ON SPUTUM CLEARANCE OF PSEUDOMONAS AERUGINOSA IN NON-CYSTIC FIBROSIS} BRONCHIECTASIS

NS HARUN ${ }^{1}$, K SANDERS ${ }^{2}$, A STUART ${ }^{2}$, CL STEINFORT

${ }^{1}$ Department of Respiratory Medicine, Barwon Health, Vic., Australia, and

${ }^{2}$ Department of Clinical and Biomedical Sciences, Barwon Health,

Vic., Australia

Aims Nebulized colistin is used to treat recurrent exacerbations of bronchiectasis due to Pseudomonas aeruginosa, a major pathogen regarded as difficult to eradicate. This case-control study aimed to establish if long-term colistin use could clear $P$. aeruginosa from the sputum of adults with non-cystic fibrosis bronchiectasis, and if so, whether colistin could be ceased in these patients. Secondary outcomes included effects of colistin on quality of life (QoL), symptom control, admission rates, lung function and tolerability.

Methods (1) Sputum was collected in 42 bronchiectasis patients with $P$ aeruginosa. Clearance rates in those on colistin were compared with a contro group not on colistin. (2) Colistin patients cleared of $P$. aeruginosa ceased treatment. Sputum was re-cultured at day 7 and 28 to detect recurrence. (3) A questionnaire assessing QoL, symptom control, and admission rates was performed on 32 patients. Outcomes were compared before and after colistin use. Long-term colistin side-effects and lung function were also assessed. Results (1) $58 \%(n=14 / 24)$ of colistin patients cleared $P$. aeruginosa from sputum compared with $44 \%(n=8 / 18)$ in the controls $(p=0.372)$. (2) $38 \%(n$ $=3 / 8$ ) of patients ceasing colistin remained free of $P$. aeruginosa at day 28 . (3) There was no difference in frequency of breathlessness, sputum production or QoL scores between the groups $(p>0.05)$. The colistin group had lower FVC (1.46 vs. $2.26 \mathrm{~L}, \mathrm{p}=0.023)$ and higher admission rates $(42 \%$ vs. $12 \%, p$ $=0.049$ ). On colistin, $69 \%$ of patients reported reduction in sputum frequency, breathlessness and improvement in QoL. Fifty percent reported decreased admission rates. There were no colistin side effects.

Conclusions Clearance of $P$. aeruginosa in sputum is possible. Clearance rates were similar in those with more severe bronchiectasis treated with colistin compared with stable patients not on colistin, and may suggest suppression of $P$. aeruginosa by colistin in this severe group. There are benefits of colistin on QoL, symptom control and admission rates. Continued sputum clearance after colistin cessation is achievable in some patients. Nebulized colistin use is well tolerated.

Nomination Janet Elder Travel Award.

Conflict of Interest No.

Journal Compilation (C 2011 Asian Pacific Society of Respirology

\section{COMMUNITY ACQUIRED RESPIRATORY VIRAL INFECTION IS A} RISK FACTOR FOR DEATH AFTER LUNG TRANSPLANTATION

A BURKE, T DANIELS, P HOPKINS, S YERKOVICH, D CHAMBERS Qld Centre for Pulmonary Transplantation \& Vascular Disease, The Prince Charles Hospital. Brisbane, QId

Background Community acquired respiratory viral (CARV) infections are believed to contribute to morbidity and mortality after lung transplantation, but previous studies have not conclusively established the evidence base in this area.

Patients and Methods A prospective cohort study was performed at a single centre from August 1998 to March 2010 ( $n=201$ lung transplant recipients). CARV infection (human metapneumovirus (hMPV), respiratory syncytial virus (RSV), influenza A (Flu A), influenza B (Flu B), adenovirus and parainfluenza virus (PIV)) was confirmed using polymerase chain reaction (PCR) of upper (nasopharangeal swab) and/or lower (bronchoalveolar lavage) respiratory tract secretions. CARV infection and BOS were included as segmented time-dependent covariates in a Cox proportional hazards model with death as the outcome variable.

Results 87 patients ( $43 \%$ of the total cohort) had a total of 147 separate CARV episodes: 42 PIV, 26 hMPV, 37 RSV, 26 Flu A, 10 Flu B, and 6 adenovirus. Infection with either RSV or hMPV was associated with an increased risk of death ( $p<0.001 \mathrm{HR} 3.6,95 \%$ confidence interval, 1.86-6.98), and the effect persisted after multivariate analysis. BOS was also a risk factor for acquiring hMPV or RSV infection ( $p=0.023$ OR $2.13,95 \%$ confidence interval, 1.11-4.11).

Conclusions Infections with hMPV and RSV, but not other CARVs, are associated with an increased likelihood of death. The presence of BOS is a risk factor for symptomatic infection with hMPV and RSV.

Conflicts of Interest None.

\section{HOSPITAL VENOUS THOMBOEMBOLISM PREVENTION PROGRAM}

S KARUNARATHNE, S BASTIAMPILLAI, J STANFIELD, CL LIEW Department of Thoracic Medicine, Royal Adelaide Hospital, South Australia

Background Venous thromboembolic events (VTE) in hospitalized medical patients cause a significant percentage of in-hospital morbidity, mortality and cost. There were 14716 VTE cases in 2008, (8253 PE, 6262 DVT) causing $\$ 1.72$ billion financial cost. Chemical (LMWH/ Unfractionated heparin) and mechanical (Graded compression stockings/ Intermitted pneumatic compressions) prophylaxis have been shown to be effective in the prevention of VTEs. However, use of such agents is suboptimal in hospital patients. This study aims to determine whether a dedicated multidisciplinary education and reinforcement program improves the use of appropriate VTE prophylaxis.

Methods Prior to the education programme, we audited a 30 bed general thoracic medical ward including patients with general medical conditions, lung cancer, chronic obstructive pulmonary disease, lung transplant and Cystic Fibrosis. Our multidisciplinary research team developed and implemented an education program over 3 months, using posters, leaflets and oral presentations to increase awareness and promote adherence to VTE prophylaxis guidelines for health care staff involved in direct patient management. Following completion of the program, we reaudited the same 30 bed ward.

Results Prior to the education program, a total of 21 patients (mean age 60 $\pm 10)$ were identified as appropriate for VTE prophylaxis. Of these $14(66 \%)$ were on appropriate VTE prophylaxis. The post education audit showed 22 out of $23(96 \%)$ patients were on appropriate VTE prophylaxis. $(p=0.013)$. Conclusion An effective multi-faceted educational program can improve delivery of appropriate VTE prophylaxis, leading to improved outcomes in hospitalized patients.

Supported by Sanofi Aventis.

Conflict of Interest Nil. 
BONE DENSITY IN LUNG TRANSPLANT PATIENTS: A 10 YEAR REVIEW

S BASTIAMPILLAI, MD HOLMES, MJ KELLY, CL LIEW

Department of Thoracic Medicine, Royal Adelaide Hospital, South Australia

Introduction Osteoporosis is common in patients with end-stage lung disease, due the underlying disease, immobility, hypoxia, prior medications. Following lung transplantation (LTx), bone loss is accelerated; osteoporosis and non-traumatic fractures are associated with morbidity. The purpose of this study is to determine the prevalence of osteoporosis and osteopenia in recipients prior to LTx and assess trends in bone loss post-LTX.

Methods Between January 2000 and January 2010, 70 South Australian patients received LTx. Bone mineral density (BMD) was assessed by dualenergy X-ray absorptometry (DEXA). Electronic databases and medical records were retrospectively analysed to determine BMD by lumbar spine $\mathrm{T}$-score, femur T-score, immunosuppression dosage and therapeutic medication intervention.

Results Pre-LTx DEXA was available in 43 of 70 pts (61\%). 37 out of 70 (53\%) patients had post-LTx DEXA within a mean time of 20 months post-LTx. Mean age at LTx was 44 years. Male:female. 38:32 patients. Immunosuppression consisted of prednisolone, calcinuerin inhibitor and cell cycle inhibitor. Mean dose of prednisolone at time of post-LTx BMD was $12 \mathrm{mg}$ (range 5-50 mg). Average pre-LTx lumbar T score was -1.29 and femur T score -1.32 . $70 \%$ of patients who had pre-LTx DEXA had T scores within the osteopenic or osteoporotic range. Average lumbar and femur $\mathrm{T}$ scores fell to -1.86 and -1.87 , respectively $(p<0.05)$, a significant difference despite the majority of patients with osteoporosis receiving combinations of calcium, vitamin D and bisphosphonate. Pre LTx mean lumbar and femur T scores in pts aged $<50$ years was -0.99 and -1.04 , respectively, and in pts over 50 years, mean lumbar and femur $T$ scores was -1.57 and -1.56 .

Conclusions Osteoporosis or osteopaenia is present in the majority of patients pre-LTx highlighting the need for early intervention. BMD worsens post-LTx despite intervention with combinations of calcium, vitamin D and bisphosphonate. Age is an important predictive factor for BMD in patients prior transplant.

Grant Support Nil.

Conflict of Interest No. \\ PAEDIATRIC SIG: POSTER SESSION}

\section{ARE MATERNAL ASTHMA EXACERBATIONS DURING PREGNANCY RELATED TO IMPAIRED INFANT GROWTH IN THE FIRST SIX MONTHS OF LIFE?}

J MATTES ${ }^{1,2,3}$, V MURPHY $Y^{2,4}, \mathrm{~K} \mathrm{MCLAUGHLIN}^{2,4}, \mathrm{~K} \mathrm{STEEL}^{2,4}$, H POWELL ${ }^{2,4}$, P GIBSON ${ }^{2,4}$

${ }^{1}$ Experimental\&Translational Immunology Group, ${ }^{2}$ Priority Research Centre for Asthma and Respiratory Disease, The University of Newcastle, NSW, Australia, ${ }^{3}$ Paediatric Respiratory Sleep Unit, John Hunter Children's Hospital, and ${ }^{4}$ Department of Respiratory \& Sleep Medicine, HMRI, John Hunter Hospital, NSW, Australia

Objective To determine the impact of maternal asthma exacerbations during pregnancy upon growth and respiratory illnesses in early life.

Methods Infants born to mothers with and with asthma have a clinical assessment at 6 and 12 months of age. Parents fill in a standardized health questionnaire each 6 months as well as prospective diary.

Results Analysis of data from 120 infants followed up to date shows that wheezing is more common in infants born to asthmatic as compared to nonasthmatic mothers at 6 months of age $(12 \%$ vs. $40 \%$; $p<0.01)$. Weight was lower in infants born to mothers with asthma as compared to those without asthma at 6 months of age $(8.0 \mathrm{~kg}$ vs. $7.5 \mathrm{~kg}$; p < 0.05). Impairment of weight gain was most pronounced in 6 months old infants from asthmatic mothers that had exacerbations during pregnancy as compared those that had stable asthma $(7.7 \mathrm{~kg}$ vs. $7.2 \mathrm{~kg}$; $\mathrm{p}<0.05)$.

Conclusions Asthma exacerbations during pregnancy may impair the infant's normal growth in the first six months of life, which could have important implications upon healthy lung development in early life.

Support NHMRC Fellowship, Hunter Medical Research Institute (HMRI), Hunter Children's Research Foundation.

Conflict of Interest Nil.
PULMONARY TOXICITY OF BIOLOGICAL ANTI-INFLAMMATORY AGENTS - A SYSTEMATIC REVIEW

BS RAO ${ }^{1}$, JEFF BOWDEN ${ }^{1}$, KARIN NYFORT-HANSEN ${ }^{2}$

${ }^{1}$ Respiratory Department, Flinders Medical Centre, SA, and ${ }^{2}$ Pharmacy

Flinders medical Centre/Repatriation General Hospital, SA

Introduction The anti-rheumatic anti-inflammatory biological agents in clinical use are abatacept, anakinra, adalimumab, etanercept, infliximab and rituximab. A variety of pulmonary side-effects have recently been reported for these agents and the purpose of this review is to compile the various reported pulmonary toxicities and their prevalence

Methods We performed a search of databases OVID MEDLINE $®$ and EMBASE of the English literature up to August 2010 using the MESH terms of abatacept, anakinra, rituximab, adalimumab, etanercept, infliximab and respiratory tract disease with limits to include only human studies or case reports. In addition case reports of respiratory adverse effects reported to the Australian Drug Reaction Advisory Committee (ADRAC) were obtained in order to identify the most common pulmonary reactions reported with each individual agent

Results Using the search criteria defined above 110 and 237 articles were identified in the OVID MEDLINE and EMBASE database respectively. The majority of ADRAC reports were associated with rituximab $(n=108)$ and infliximab $(n=107)$, followed by adalimumab $(n=43)$ and etanercept $(n=43)$. Various pulmonary side-effects including interstitial lung disease associated with anti-inflammatory agents were identified.

Discussion From the articles reviewed, details about the duration between onset of treatment and incidence of pulmonary side effects, diagnosis, treatment options and outcome of patients were extracted and are presented here. Conclusion This comprehensive systematic review hopes to improve the awareness about the serious and potentially life-threatening pulmonary sideeffects of this group of agents.

Conflict of Interest No.

\section{AREA UNDER THE REACTANCE CURVE: REFERENCE VALUES} AND DISEASE DETECTION IN CHILDREN BORN PRETERM

SJ SIMPSON ${ }^{1}$, PD SLY ${ }^{1}$, P FRANKLIN ${ }^{2}$, E LOMBARDI $^{3}$, C CALOGERO $^{3}$ $M^{2}$ PALUMBO $^{4}$, GL HALL ${ }^{1,5}$

${ }^{1}$ Telethon Institute for Child Health Research, Perth, WA, ${ }^{2} \mathrm{SPACH}$, University of WA, ${ }^{3}$ Anna Meyer University Hospital for Children, Italy, ${ }^{4}$ Belcolle Hospital, Italy, and ${ }^{5}$ Respiratory Medicine, Princess Margaret Hospital for Children, Perth, WA

Introduction The forced oscillation technique (FOT) is effort independent and thus ideal for young children. The area under the reactance curve $(A X)$ has been proposed to amplify clinically relevant signal by taking advantage of any shape change in the reactance (Xrs) curve below the resonant frequency. This study aimed to develop reference values for resistance (Rrs), Xrs and AX in a large healthy population of children, and determine if AX conferred any additional clinical benefit when examining disease in children born preterm.

Methods Impedance spectra were obtained in 760 healthy children (335 male), aged less than 13 years and with height less than $160 \mathrm{~cm}$ using a commercial device (I2M, Chess Medical, Belgium). AX was calculated in 647 of these children between $6 \mathrm{~Hz}$ and the resonant frequency. Backwards stepwise linear regressions identified the best predictors of $A X$, and Xrs and Rrs at $8 \mathrm{~Hz}$ $\left(\mathrm{Xrs}_{8}, \mathrm{Rrs}_{8}\right)$, and $\mathrm{Z}$ scores were generated. Z scores were calculated for 156 children born preterm, of which 87 received a neonatal diagnosis of bronchopulmonary dysplasia (BPD). Chi squared tests examined the difference in proportion of children born preterm (with and without BPD) with abnormal Z scores for each FOT variable.

Results All FOT variables were predicted by height $(p<0.001)$ and sex. Mean (SD) Z scores for preterm children with and without BPD for Rrs 8 (0.59 (0.88); $0.58(1.03)), \mathrm{Xrs}_{8}(1.05(0.68) ; 0.61(0.82))$ and $\mathrm{AX}(0.84(0.70) ; 0.51$ $(0.98))$ were all significantly different $(\mathrm{p}<0.001)$ from the healthy population. The number of children born preterm with abnormal $Z$ scores was not significantly different when comparing $\mathrm{AX}, \mathrm{Rrs}_{8}$ and $\mathrm{Xrs}_{8}$.

Conclusions While $\mathrm{AX}$ is able to detect respiratory disease in preterm children with and without BPD, it is no more sensitive than Xrs or Rrs.

Supported by PMH Foundation, NHMRC, Asthma Foundation WA, CaRiVit, NGO 'Solidarietà e Servizio' Viterbo.

Conflict of Interest No.

Journal Compilation @ 2011 Asian Pacific Society of Respirology 


\section{LUNG FUNCTION OUTCOMES IN CHILDREN BORN $\leq 32$ WEEKS GESTATION}

\author{
KM LOGIE ${ }^{1}$, SJ SIMPSON ${ }^{2}$, J GIBBONS ${ }^{3}$, AC WILSON $^{1}$, JJ PILLOW WI, $^{3}$ \\ GL HALL ${ }^{1,2}$ \\ ${ }^{1}$ School of Paediatrics and Child Health and ${ }^{3}$ School of Women's and \\ Infants' Health, University of Western Australia, and ${ }^{2}$ Telethon Institute for \\ Child Health Research, Perth, WA
}

Introduction Standardization of current treatments such as surfactant steroid and ventilation use in the 1990s has meant a shift in the respiratory pathology of preterm birth particularly, bronchopulmonary dysplasia (BPD). Consequently we now lack thorough understanding of current respiratory outcomes of preterm birth.

Aim To quantify lung function outcomes in children born $\leq 32$ weeks gestation both with a neonatal diagnosis of BPD (requiring $\geq 28$ days supplemental oxygen) and those without a neonatal diagnosis of BPD.

Methods Children aged 9 to 11 years performed lung function tests, including; forced oscillation, static lung volumes, spirometry and gas transfer ( $\left.D L_{c o}\right)$. Past and recent respiratory symptoms were recorded and a chest CT and maximal cardiopulmonary exercise test were also performed.

Results To date 32 term control children and 68 children born $<32$ weeks gestation, 42 with a neonatal diagnosis of BPD, have been studied. Static lung volumes were not different between the groups. Children with BPD had significantly reduced $D L_{c o}(p=0.025)$, forced expiratory volume in the first second $\left(F E V_{1}\right)(p<0.001)$ and forced expiratory flow $\left(F F_{25 \%-75 \%}\right)(p<0.001)$. Multivariate analysis determined that the decrease in $\mathrm{FEV}_{1}$ and $\mathrm{FEF}_{25 \%-75 \%}$ was more strongly associated with gestational age $(p=0.014)$ than neonatal lung disease (days on $\mathrm{O}_{2}, \mathrm{p}=0.055$ ).

Conclusions Pre-pubertal children born preterm with a diagnosis of BPD, exhibit reduced lung function. Specifically lower $\mathrm{FEV}_{1}, \mathrm{FEF}_{25 \%-75 \%}$ and $\mathrm{DL}$ despite static lung volumes being comparable to term controls.

Supported by NHMRC, Princess Margaret Hospital Foundation.

Conflict of Interest None.

\section{PREDICTORS OF POST-OPERATIVE SORE THROAT IN INTUBATED CHILDREN}

A CALDER ${ }^{1}$, M HEGARTY $^{1}$, TO ERB ${ }^{2}$, BS VON UNGERN-STERNBERG ${ }^{1,3}$ ${ }^{1}$ Department of Anaesthesia, Princess Margaret Hospital for Children, Perth, Australia, 'Division of Anaesthesia, University Children's Hospital, Basel, Switzerland, and ${ }^{3}$ School of Medicine and Pharmacology, University of Western Australia, Perth, Australia

Aim To investigate factors influencing the incidence of sore throat in children undergoing elective day case surgery with an endotracheal tube (ETT).

Methods 500 children (3-16 years) were prospectively studied. The choice of the ETT (cuffed or uncuffed) was left to the attending anaesthetist who was unaware of the purpose of the study. In the cases cuffed ETT use, the cuff was inflated to the clinical end point of loss of an audible leak as determined by the anaesthetist. Cuff pressure (CP) was then measured using a calibrated cuff manometer. Chi-square testing and stepwise logistic regression were used to determine predictors of post-operative sore throat.

Results $111(22 \%)$ of children developed a sore throat, $19(4 \%)$ a sore neck and $5(1 \%)$ a sore jaw. The ETT CP, the use of uncuffed ETTs and ENT surgery were univariate predictors of post-operative sore throat. Overall, $19 \%$ of patients with cuffed ETTs complained of sore throat compared with $37 \%$ of those intubated with an uncuffed ETT. In patients with cuffed ETT, no patient complained of a sore throat when the $\mathrm{CP}$ was $0-10 \mathrm{cmH} 2 \mathrm{O}$. The incidence of sore throat increased as CP increased; $4 \%$ at $11-20 \mathrm{cmH} 2 \mathrm{O}, 20 \%$ at $21-30 \mathrm{cmH} 2 \mathrm{O}, 68 \%$ at $\mathrm{CP} 31-40 \mathrm{cmH} 2 \mathrm{O}$ and $96 \%$ at $\mathrm{CP}>40 \mathrm{cmH} 2 \mathrm{O}$. Conclusions The use of uncuffed ETTs as compared with cuffed ETTs, ear, nose and throat surgery and CP were predictors of post-operative sore throat We demonstrated that CPs of ETTs were closely related to the development of sore throat with higher pressures increasing the likelihood. Therefore, when using cuffed ETTs, CP should be routinely measured intra-operatively. Conflict of Interest No.

\section{TP-133}

GAS TRANSFER, PULMONARY CAPILLARY BLOOD VOLUME, AND PULMONARY MEMBRANE DIFFUSION IN CHILDREN BORN PRETERM

JTD GIBBONS ${ }^{1}$, KM LOGIE ${ }^{1}$, AC WILSON ${ }^{1}$, JJ PILLOW ${ }^{2}$, GL HALL $^{3}$ ${ }^{1}$ School of Paediatrics and Child Health, University of Western Australia, WA, Australia, ${ }^{2}$ School for Women's and Infants' Health, University of Western Australia, WA, Australia, and ${ }^{3}$ Telethon Institute for Child Health Research, WA, Australia

Introduction Survivors of preterm birth born with bronchopulmonary dysplasia (BPD) in the pre-surfactant era of neonatal care (classical BPD) have a reduced pulmonary gas transfer capacity. There is, however, little data to describe gas transfer in preterm infants with BPD in the post-surfactant era (new BPD).

Objective Assess gas transfer using carbon monoxide diffusing capacity $\left(\mathrm{DL}_{\mathrm{co}}\right)$ and its components, pulmonary capillary blood volume $(\mathrm{Vc})$ and pulmonary membrane diffusion $\left(D_{M}\right)$, in contemporary survivors of preterm birth. Method Gas transfer was assessed using single-breath $\mathrm{DL}_{\mathrm{co}}$ in children aged 9 to 11 years and born $<32$ weeks gestation with $B P D(P B, n=15)$ and without $B P D(P T, n=20)$, and in term born controls $(T C, n=27)$. $D L_{c o} Z$ scores were calculated. $\mathrm{D}_{\mathrm{M}}$ and $\mathrm{V}_{\mathrm{C}}$ were determined in $12 \mathrm{~PB}, 12 \mathrm{PT}$ and $25 \mathrm{TC}$ children.

Results The mean (SD) $D L_{c o} Z$ score for the PB group was $-0.58(0.89)$ differing significantly from $0(p=0.03)$ while the PT and TC groups $(0.01(1.02)$ and $-0.12(0.94)$, respectively) did not $(p>0.05)$. $D_{M}$ was lower in the PB group than the PT and TC groups, with no difference between PT and TC groups. Differences in $D_{M}$ were not significant after adjusting for lung size. There were no differences in $V_{c}$ between groups.

Conclusion Gas transfer is reduced in survivors of preterm birth with new $B P D$. The tendency for reduced $D_{M}$ and not $V_{c}$ in children with new BPD suggests that impaired gas transfer may be a result of alterations in the alveolar membrane rather than pulmonary vascular function.

Supported by NHMRC, Raine Foundation and PMH Foundation. Conflict of Interest No.

Journal Compilation (C) 2011 Asian Pacific Society of Respirology

\section{IMPROVING ENROLMENT AND RETENTION OF MAORI AND PACIFICA FAMILIES IN RESPIRATORY RESEARCH}

\section{MOBBERLEY ${ }^{1,2}$, S KAKE $^{1}$, H FAASALELE $^{3}$, J PATERSON ${ }^{4}$,} CA BYRNES ${ }^{1,2}$

${ }^{1}$ Department of Paediatrics, University of Auckland, ${ }^{2}$ Starship Children's Hospital, ${ }^{3}$ Pacific Island Support Unit, and ${ }^{4}$ Kiatewhai Auckland City Hospital, Auckland

Background Bronchiectasis is common in indigenous populations such as Alaska Natives, Australian Aboriginal, and New Zealand Maori and Pacifica. As part of an international collaborative interventional study, we sought the participation of Maori and Pacifica families - groups difficult to engage in research in the past.

Aim To engage, enrol and retain children from Maori and Pacifica families from Auckland in a 2-year research study.

Methods A randomized controlled trial to determine whether azithromycin is superior to placebo in reducing exacerbations seeking to enrol children aged 12 months to 8 years with bronchiectasis. The enrolment procedure was modified to a process deemed more appropriate to these cultures: (1) Request to defer the decision of enrolment until the process had been completed. (2) A minimum of 3 meetings; initial invitation, discussion in the home with the extended family, invitation to the extended family to participate in the day of enrolment. (3) Appointment of a 'whanau worker' (family worker) to sit with the family and empower them to get all the information they seek prior to enrolment.

Results Of 39 families approached, $38(97 \%)$ children (median age 5.4 years, range $0.8-8.9$ years) enrolled with $26 \%$ Samoan, $23 \%$ Tongan, $16 \%$ Maori and $35 \%$ mixed Maori/Pacifica heritage. After 1-year retention was 34 (89\%) with 1 exiting the study after 1 month with new non-pulmonary disease, and 3 exiting after 1 year, 2 moving outside study area.

Conclusions These are high enrolment and retention figures reported in this population. We believe that following a prolonged procedure for enrolment, involving the extended family and appointing a worker to sit 'alongside' the family will improve their understanding of a research project and allow them to feel more comfortable about participating.

Conflict of Interest No.

Support Auckland Medical Research Foundation \& NZ Health Research Council. 


\section{THE EFFECTS OF GROWTH HORMONE ON SLEEP DISORDERED BREATHING IN CHILDREN WITH PRADER-WILLI SYNDROME}

M VANDELEUR $^{1}$, MJ DAVEY $^{1}$, GM NIXON $^{1,2}$

${ }^{1}$ Melbourne Children's Sleep Centre, Monash Children's, Southern Health, Melbourne, Australia, and ${ }^{2}$ The Ritchie Centre, Monash Institute of Medical Research, Monash University, Melbourne, Australia

Aim Growth hormone (GH) is licensed for children with Prader-Willi Syndrome (PWS) to improve linear growth and body composition. There have been case reports of sudden death in patients with PWS on GH occurring mainly during sleep. The aim of this study was to evaluate the impact of commencement of GH on sleep parameters in a cohort of children with PWS.

Methods We retrospectively identified all children with PWS referred for overnight polysomnography (PSG) between 2003-2010 to evaluate for sleep disordered breathing, pre and post commencement of GH. Data were compared with paired Student's $t$-test.

Results Since 2003, 24 children with PWS have been referred for consideration of GH treatment. 10/24 children were diagnosed with OSA on the initial study and GH was deferred in 8 cases. To date 11 patients with paired data have been reviewed. The mean age was 5.4 years (range .25-15.6 years). The mean obstructive apnoea hyponoea index (OAHI) did not change significantly between post $(1.7 \pm 1.9 /$ hour $)$ and pre GH $(0.7 \pm 1 /$ hour $)(p=0.3)$ however the mean central apnoea hyponoea index (CAl) significantly improved post GH ( $2.6 \pm 1.5 /$ hour $)$; pre GH $(3.6 \pm 4.2 /$ hour $)(p=0.05)$. In 3 cases OSA was not present at baseline nor post GH (3-4 months) but developed later during treatment (7 months-3.25 years).

Conclusion In this cohort of children with PWS the OAHI pre and post GH was similar but there was a significant improvement in the CAl. Three children developed significant OSA after 7 months -3.25 years on $\mathrm{GH}$. This study highlights that OSA may develop late in GH treatment. Children should be monitored for symptoms of OSA throughout GH treatment and PSG repeated if symptoms develop.

Supported by Nil.

Conflict of Interest No.

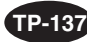

\section{ROLE OF UPPER RESPIRATORY VIRUSES IN EXACERBATIONS} OF NON-CYSTIC FIBROSIS BRONCHIECTASIS IN CHILDREN

N KAPUR ${ }^{1}$, IM MACKAY ${ }^{2}$, IB MASTERS ${ }^{1}$, TP SLOOTS ${ }^{2}$, AB CHANG ${ }^{1}$

'Department of Respiratory Medicine, Royal Children's Hospital, Qld, and

${ }^{2}$ Queensland Paediatric Infectious Diseases Laboratory, SASVRC, QId

Respiratory viral infections often lead to exacerbations of chronic respiratory diseases such as asthma and COPD though there is no similar data in noncystic fibrosis (CF) bronchiectasis. The objectives of our study were to (1) determine the point prevalence and identify viruses associated with exacerbations and (2) evaluate clinical and investigational differences between viral infection positive and negative exacerbations in children with non-CF bronchiectasis.

Methods A cohort of 69 children (median age 7 years; 33 boys) with non-CF bronchiectasis was prospectively followed for 900 child-months. Polymerase chain reaction for 16 respiratory viruses was performed on nasopharyngeal aspirates collected during 77 paediatric pulmonologist defined exacerbations. Data on clinical, parent cough-specific quality of life (PC-QOL), systemic markers (CRP, IL6, procalcitonin, amyloid-A, fibrinogen) and lung function parameters were also collected.

Results Respiratory viruses were detected during 37(48\%) exacerbations: picornavirus in 24 episodes [Human-rhinovirus (HRV) in 20, enterovirus in 4]; Human Bocavirus in 4; adenovirus, Human meta-pneumovirus, influenza A, respiratory syncytial virus, parainfluenza 3 and 4 in two each; coronavirus and parainfluenza 1 and 2 in one each. Viral co-detections occurred in $6(8 \%)$ exacerbations. Among 14 genotyped HRV's, more HRV-A's $(n=9)$ were identified than HRV-C's $(n=2)$. Children with proven viral infections were more likely to have fever (OR $3.8,95 \% \mathrm{Cl} 1.1-13.3$ ), wheeze and/or crackles (OR 3.4, $95 \% \mathrm{Cl} 1.3-8.7$ ) and raised CRP (OR 4.8, 95\% Cl 1.8-12.9) when compared with virus negative exacerbations. There were no other statistically significant differences.

Conclusions Respiratory viruses are commonly found during pulmonary exacerbations in children with non-CF bronchiectasis. HRV-A is the most frequently detected virus. Time sequenced cohort studies during stable state, exacerbations and recovery periods are needed to determine the importance of viral infections and their possible interaction with bacteria.

Supported by ANZ Trustees scholarship.

Conflict of Interest None.

Nominations None.

\section{RANDOMIZED CONTROLLED TRIAL TO REDUCE MORDIBITY OF BRONCHIOLITIS IN YOUNG CHILDREN ADMITTED TO ROYAL DARWIN HOSPITAL IN THE NT}

G MCCALLUM ${ }^{1}$, L VERSTEEGH ${ }^{1}$, C MACLENNAN $^{2}$, C WILSON $^{1}$, S PIZZUTTO' ${ }^{1}$, PS MORRIS ${ }^{1,2}$, AB CHANG $^{1,3}$

${ }^{1}$ Child Health Division, Menzies School of Health Research, NT,

${ }^{2}$ Department of Paediatrics, Royal Darwin Hospital, NT, and ${ }^{3,1}$ Respiratory

Medicine, Royal Children's Hospital, Brisbane, Qld

Aim Bronchiolitis is the most common reason for hospital admission for infants globally (1). The use of macrolides for treating bronchiolitis in nonaffluent settings remains controversial but potentially beneficial. In our region readmission with lower respiratory illness in young children (particularly indigenous children) remains high. This RCT aims to determine if a single dose of azithromycin reduces the morbidity of young children with bronchiolitis.

Methods Double blinded RCT. Young children $\leq 18$ months admitted to Royal Darwin Hospital $(\mathrm{RDH})$ diagnosed with bronchiolitis are eligible. Children are given a single dose $(30 \mathrm{mg} / \mathrm{kg})$ of either azithromycin/placebo. Primary outcome is length of stay for respiratory disease. Secondary outcomes are duration of oxygen use and readmission for respiratory illness in 6-month period.

\begin{tabular}{|c|c|c|c|c|c|c|}
\hline \multirow{2}{*}{$\begin{array}{l}\text { Mean }_{\text {hrs }} \\
\text { (SD) }\end{array}$} & \multirow{2}{*}{$\begin{array}{c}\text { Indigenous } \\
(\mathrm{n}=42)\end{array}$} & \multirow{2}{*}{$\begin{array}{c}\text { Non- } \\
\text { Indigenous } \\
(n=16)\end{array}$} & \multicolumn{4}{|c|}{ Medication groups } \\
\hline & & & A & $\mathrm{B}$ & C & $\mathrm{D}$ \\
\hline $\mathrm{O}_{2}$ & $61.2(68.4)$ & $44.9(34.4)$ & $72.2(104.6)$ & $52.2(34.0)$ & 44.3 & 54.4 (38.3) \\
\hline LOS & $91.3(94.7)$ & $59.8(35.4)$ & $119.7(147.0)$ & 70.4 & $61.7(21.9)$ & $71.8(37.1)$ \\
\hline
\end{tabular}

Results To date 58 children enrolled, $34 \%$ RSV+ve. Median age 5.7 months. Fifty percent have had at least one co-morbidity. Readmission rate $=18 \%$.

Conclusion Co-morbidities are high in this population. Antibiotics have the potential to help reduce the impact of additional respiratory burden.

Supported by Financial Markets Foundation for Children \& Channel 7 Foundation.

Conflict of Interest None

Reference

(1) Chang AB, Chang CC, O'Grady K, Torzillo PJ. Lower respiratory tract infections. Pediatr Clin North Am 2009 Dec; 56 (6):1303-21.

TP-139

AN UNUSUAL INHALED FOREIGN BODY IN TWO CHILDREN: A CASE SERIES

A WITHERS ${ }^{1}$, D COX ${ }^{1}$, F LANNIGAN ${ }^{2}$, S STICK $^{1}$

${ }^{1}$ Department of Paediatric Respiratory Medicine, Princess Margaret Hospital, Perth, Australia, and ${ }^{2}$ Department of Paediatric ENT Surgery, Princess Margaret Hospital, Perth, Australia

Introduction Foreign body inhalation is a relatively common presentation in young children, especially less than 3 years of age. Early recognition remains a critical factor in the treatment of foreign body inhalation in children. Inhaled foreign bodies in children are most often organic material, with seeds and peanuts being the most common items. On review of the literature, there are very few case reports of inhaled metal screws. We report two unusual cases of inhaled metal screws that presented to our service.

Case Presentation Both cases presented to our emergency department with wheeze, respiratory distress and fever. Foreign body inhalation was not considered as a cause for their symptoms until the object was identified on chest $x$-ray. Both foreign bodies were removed successfully but one child required invasive ventilation in our intensive care unit post removal. Both children made a full recovery. Interestingly, both metal screws came from flat pack furniture purchased from a well known international home products store.

Conclusion Foreign body inhalation must always be considered as a cause of respiratory distress in a child. With the increase in the number of flat pack furniture in Australian home's, we believe parents must be warned of the potential danger of loose metal screws to young children.

Supported by None.

Conflict of Interest None.

Journal Compilation (c) 2011 Asian Pacific Society of Respirology 


\section{COUGH IS POORLY REPORTED BY CARERS OF ABORIGINAL CHILDREN}

MJ MOREY ${ }^{1,2}$, AC CHENG $^{1,3}$, AB CHANG $^{1,4}$

${ }^{1}$ Menzies School of Health Research, Charles Darwin University, NT, ${ }^{2}$ Faculty of Medicine, Dentistry and Health Sciences, University of Melbourne, Vic., ${ }^{3}$ Department of Epidemiology and Preventive Medicine, Monash University, Vic., and ${ }^{4}$ Department of Respiratory Medicine, Royal Children's Hospital, Qld

Aims and Objectives To determine the reliability of a 24-hour history of reported cough presence and quality (wet/dry) compared to objectively recorded cough, and evaluate factors that may influence cough reporting. Methods A digital recorder was attached to 47 Indigenous children for 24 hours during admission at the Royal Darwin Hospital. After recording, carers reported their child's cough details. Cough counts objectively measured were compared with details of cough reports by carer (cough present or absent, quality wet or dry and cough severity determined by visual analogue scale and verbal category descriptive score).

Results Reporting of cough by Indigenous carers' is poor $(\mathrm{K}=0.17,95 \% \mathrm{Cl}$ $-0.15,0.49)$ and still poor when a higher cough threshold was used ( $\mathrm{K}=0.57$, $95 \% \mathrm{Cl} 0.28,0.88)$. Carers' evaluation of wet cough disagreed with clinician's evaluation $(\mathrm{K}=-0.24,95 \% \mathrm{Cl}-0.58,0.09)$. Subjective reporting of cough severity correlated to objective cough rates but not well $\left(r_{\mathrm{s}}=0.41\right.$ to $0.44,95 \%$ $\mathrm{Cl} 0.11,0.67$ ). None of the factors examined (sex, age of carer, smoking history of carer, relationship to child, concerns of the carer) was associated with reliability of cough reporting.

Conclusions The unreliability of reporting of cough highlights the need for community education on the importance of cough to overcome the perception that having a cough is normal, so as to improve health-seeking behaviour for early detection and treatment of respiratory disease. Also health professionals should not rely solely on reported history in these settings.

Conflict of Interest None.

\section{TP-141}

THE DIAGNOSTIC YIELD OF FLEXIBLE BRONCHOSOPY WITH BRONCHIAL ALVEOLAR LAVAGE IN THE MANAGEMENT OF PAEDIATRIC PATIENTS WITH FEBRILE NEUTROPENIA

JKW WONG, GJ SMITH, A TAI

Department of Respiratory Medicine and Sleep Medicine, Women's and Children's Hospital, SA, Australia

Aim To determine the role of flexible bronchoscopy with bronchial alveolar lavage (BAL) in the management of patients with febrile neutropenia.

Methods A retrospective analysis was made of the number of patients admitted with febrile neutropenia at a single institution who underwent bronchoscopy plus BAL from years 2004 to 2010. Computer database plus patient case notes were reviewed to establish clinical symptoms and signs, radiological findings, antimicrobial treatment and mean duration to bronchoscopy following admission.

Results A total of 668 episodes of febrile neutropenia were recorded years 2004 to 2010. Seven patients (3 males and 4 females) were referred for bronchoscopy plus BAL. The mean age was 10.1 years (age range $4-14$ years) and all had been diagnosed with acute lymphoblastic leukemia. All patients had at least cough as a clinical symptom along with radiological findings. All patients had been on broad spectrum antibiotics at the time of bronchoscopy. The mean duration from admission to time of bronchoscopy was 216 hours (9 days) with a standard deviation of 139 hours. Of the seven patients one patient yielded a positive result on BAL. This did not result in a change in management as the patient improved clinically before the result of the BAL was confirmed.

Conclusion In this retrospective case series the diagnostic yield of flexible bronchoscopy plus BAL in children with febrile neutropenia was low. Prospective studies plus early timing towards bronchoscopy and BAL should be conducted to further define its role in the management of febrile neutropenic patients.

Conflict of Interest Nil.

Journal Compilation (C 2011 Asian Pacific Society of Respirology

\section{MULTICENTRE RCT USING STANDARDIZED MANAGEMENT ALGORITHM FOR TREATMENT OF CHRONIC COUGH IN CHILDREN}

C WILLIS ${ }^{1}$, CF ROBERTSON ${ }^{2}$, PP VAN ASPEREN ${ }^{3}$, PS MORRIS ${ }^{4}$ JM MARCHANT ${ }^{1}, \mathrm{~N} \mathrm{GLASGOW}^{5}$, L TEOH ${ }^{6}$, IBMASTERS ${ }^{1}$, CM MELLIS $^{7}$, LI LANDAU ${ }^{8}, A B$ CHANG $^{1,4}$

Departments of Respiratory Med at ${ }^{1}$ Royal Children's Hospital, Brisbane,

${ }^{2}$ Royal Children's Hospital, Melbourne, ${ }^{3}$ Children's Hospital Westmead, Sydney, ${ }^{6}$ The Canberra Hospital, Canberra, and ${ }^{4}$ Menzies School of Health Research, Darwin, ${ }^{8}$ University of Western Australia, Perth, ${ }^{7}$ University of Sydney, ${ }^{5}$ Australian National University, Canberra

Cough in children is a common symptom. Data on causes of chronic cough in young children have previously been published by our units. However, differences in underlying diagnosis by age at presentation have not been assessed. We present the 'time to cessation' of cough in our multicentre RCT using a standardized management algorithm in newly referred children with chronic cough ( $>4$ weeks) from 7 Australian centres.

Methods Parents completed validated cough diary and cough specific QOL (PC-QOL) at recruitment and at cessation of cough. The diagnosis made by the treating physician was based on 2006 TSANZ Position Statement.

Results The median (range) age of the 226 children recruited was 3.3 years $(0.2-14.2) ; 131(58 \%)$ were boys. Median (IQR) PC-QOL post treatment of 6.5 $(5.1,6.9)$ improved significantly $(p=0.0001)$ from $3.8(2.8,5.0)$ at enrolment. The median (IQR) duration of cough at recruitment was 16 weeks $(8.0,48.5)$ and 'time to cessation' of cough after application of the management algorithm was 3 weeks $(1.0,7.0)$. There was no significant difference $(p=0.615)$ in median (IQR) 'time to cessation' of cough among the three age cohorts: $<2$ years $(n=74,32.7 \%)$ was 2.5 weeks $(0.8,7.0) ; 2-6$ years $(n=93,41.2 \%)$ was 3 weeks $(1.0,7.0)$; and $>6$ years $(n=59,26.1 \%)$ was 3 weeks $(3.0,7.0)$. There was also no significant difference in the final primary diagnosis among the three age cohorts $(p=0.09)$. The most common diagnoses were protracted bacterial bronchitis ( $n=95,42 \%)$, asthma/reactive airways disease $(n=38$, $16.8 \%)$, tracheobronchomalacia $(n=17,7.5 \%)$ and bronchiectasis $(n=16$, $7.1 \%) .76$ children (33.6\%) had more than one diagnosis.

Conclusions The aetiology and 'time to cessation' of chronic cough in children managed in accordance to a standardized pathway were similar among the three age groups. It is likely that our previous findings in very young children are also applicable to older children.

Supported by NHMRC grant number 490321.

Conflict of Interest None.

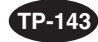

RISK FOR DEVELOPMENT OF BRONCHIECTASIS IN PATIENTS WITH PROTRACTED BACTERIAL BRONCHITIS

D WURZEL ${ }^{1}$, JM MARCHANT ${ }^{1}$, IB MASTERS ${ }^{1}$, S YERKOVICH $^{2}, \mathrm{~J} \mathrm{UPHAM}^{3}$, AB CHANG ${ }^{1}$

${ }^{1}$ Royal Children's Hospital, Brisbane, Qld Children's Medical Research Inst, ${ }^{2}$ Prince Charles Hospital, Brisbane and ${ }^{3}$ Princess Alexandra Hospital, Brisbane, Australia

Protracted Bacterial Bronchitis (PBB) is a common cause of persistent cough in children. It is characterized by moist cough lasting $>4$ weeks which resolves with 2 weeks of antibiotic therapy, and absence of specific indicators suggesting an alternate diagnosis. Recurrent episodes of PBB may be antecedent to later development of bronchiectasis.

Aim Longitudinal follow-up (over a 2-year period) of children with PBB to determine the: (1) proportion of children with recurrent PBB ( $\geq 3$ episodes per year) (2) proportion of children who develop bronchiectasis 3) airway impedance.

Methods Prospective cohort study involving monthly follow-up with caregivers. Two years post enrolment, children undergo clinical and lung function assessment (FOT). Presence of bronchiectasis is determined by physician review and radiological confirmation (when indicated). The frequency of PBB episodes is recorded over the study period.

Results Of 166 children recruited to the cohort study to date, $64 \%(107 / 166)$ were male. The median age at recruitment was 23 months (IQR 12, 55). 80\% of children had recurrent PBB. Of the 20 children who have had 2-year clinical follow-up, 9 were able to perform FOT and 22\% (2/9) showed abnormalities (reactance above normal range.) 20\% (4/20) with PBB have had subsequent physician diagnosis of bronchiectasis or CSLD.

Conclusion The burden of cough in children with PBB 2 years after diagnosis remains high. Ongoing clinical follow-up of this cohort of children with PBB should provide further insight into the likelihood of progression from PBB to CSLD and bronchiectasis.

Support Financial Markets Foundation for Children (for project), Allen \& Hanburys and QCMRI (for DW), NHMRC (for JU and AC).

Conflict of Interest None. 
PLEURAL FLUID NUCLEIC ACID TESTING ENHANCES PNEUMOCOCCAL SURVEILLANCE IN CHILDREN

R STRACHAN, A CORNELIUS, G GILBERT, A MARTIN, T MCDONALD, G NIXON, S RANGANATHAN, R ROSEBY, H SELVADURAI, G SMITH, M SOTO-MARTINEZ, S SURESH, L TEOH, K THAPA, C WAINWRIGHT, A JAFFÉ ON BEHALF OF THE AUSTRALIAN RESEARCH NETWORK IN EMPYEMA (ARNIE)

Sydney Children's Hospital, Randwick, Sydney

Introduction National Streptococcus pneumoniae (SP) serotype surveillance reports only culture positive cases from sterile sites but the yield from culture is low. Polymerase chain reaction (PCR) is more sensitive in detecting $\mathrm{SP}$ in culture negative samples.

Aim To determine whether enhanced molecular surveillance in childhood empyema provides additional SP serotype information compared to national surveillance methods.

Methods Pleural fluid from children with empyema underwent culture and PCR to identify SP-targeting autolysin $(I y t A)$ and multiplex PCR to identify SP serotypes. National surveillance data were obtained from the National Notifiable Diseases Surveillance System (NNDSS) for the same time period and age groups.

Results Empyema: 89 children, 48 male, median age 3.5 (range 0.6-15.5) were recruited from 1 April 08 for 12 months. SP was cultured in 9/74 (12.2\%) in blood and $5 / 84(9.5 \%)$ in pleural fluid. SP was identified by PCR in $43 / 79$ (54.4\%). Serotypes: $1, n=4(8.9 \%) ; 3, n=9(22.2 \%) ; 19 A, n=12(26.7 \%)$; 7F7A, $n=1(2.2 \%) ; 9 V / 9 A, n=1(2.2 \%) ; 22 F / 22 A, n=2(4.4 \%)$; non-typeable, $\mathrm{n}=4(8.9 \%)$. One subject had 2 serotypes and in11 a serotype could not be established. NNDSS: 361 SP culture positive cases were reported. Serotypes: $1, n=8(2.2 \%) ; 3, n=13(3.6 \%) ; 19 A, n=131(36.3 \%) ; 7 F 7 A, n=8(2.2 \%) ;$ $9 \mathrm{~V} / 9 \mathrm{~A}, \mathrm{n}=3(0.8 \%) ; 22 \mathrm{~F} / 22 \mathrm{~A}, \mathrm{n}=17(4.7 \%)$; non-typeable, $\mathrm{n}=30(8.3 \%)$. Other serotypes were reported in 151 SP positive cases. Significant differences between empyema and NSDSS data were identified for serotypes 1 ( $p$ $<0.05)$ and $3(\mathrm{p}<0.005)$.

Conclusions The proportion of serotypes 1 and 3 were significantly higher in empyema fluid using PCR. This disease model provides additional serotype information to national surveillance data. This has important implications in monitoring replacement serotypes following the introduction of new vaccines.

Funded by GlaxoSmithKline, Belgium.

Conflict of Interest Yes.

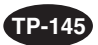

\section{INHALED CORTICOSTEROIDS FOR SUB-ACUTE COUGH IN} CHILDREN - A COCHRANE SYSTEMATIC REVIEW

S ANDERSON-JAMES ${ }^{1}$, JM MARCHANT ${ }^{2}$, K-AF O'GRADY $^{2}$, JP ACWORTH ${ }^{3}$, CT TURNER ${ }^{4}$, AB $^{\text {CHANG }}{ }^{1}$

${ }^{1}$ Queensland Children's Respiratory Centre, ${ }^{2}$ Queensland Children's Medical Research Institute and ${ }^{3}$ Department of Emergency Medicine, Royal Children's Hospital, Brisbane, QId, Australia, and ${ }^{4}$ School of Nursing and Midwifery, University of Queensland, Brisbane, Qld, Australia.

Cough is the most common symptom presenting to doctors. Paediatric cough is associated with significant morbidity for both children and their parents. The symptom of cough is associated with airway hyper-reactivity and is a dominant symptom of airway inflammation. Inhaled corticosteroids (ICS) can reduce airway inflammation and hyper-reactivity. The objective of this review was to evaluate evidence for the efficacy of ICS in reducing the severity of cough in children with sub-acute cough (defined as cough duration of 2-4 weeks).

Methods Search was conducted by the Cochrane Airways Group using Cochrane methodology. All randomized controlled trials (RCTs) comparing ICS with a control group for treatment of sub-acute cough in children were considered for inclusion. Search results were analysed using pre-determined criteria for inclusion.

Results Two studies were eligible for inclusion in the review, however there were limitations in that the participants of both these studies were infants, post acute bronchiolitis illness, and cough duration at start of study treatment was ill-defined. 98 children were included in the meta-analysis. There was no significant difference between groups in proportion of children 'not cured' (primary outcome measure), with a pooled OR of 0.61 (95\% Cl $0.24,1.55$ ) (using intention to treat analysis).

Conclusions There is currently no evidence to support the use of ICS in sub-acute cough in children. However, this systematic review is limited by the small number of studies available for analysis and the quality and design of these studies. Further well-designed RCTs are required to support or refute the efficacy of treatment with ICS in children with sub-acute cough.

Supported by Queensland Children's Medical Research Institute (QCMRI). Conflict of Interest No.
PULMONARY PHYSIOLOGY \& SLEEP SIG: POSTER SESSION

\section{RESPIRATORY FUNCTION AND VENTILATORY RESPONSE IN SUBJECTS WITH PARKINSON'S DISEASE}

H GIDDINGS ${ }^{1}$, L SECCOMBE ${ }^{1}$, P ROGERS ${ }^{1}$, A CORBETT ${ }^{2}$, E VEITCH ${ }^{1}$

${ }^{1}$ Thoracic Medicine and ${ }^{2}$ Neurology, Concord General Hospital, NSW,

\section{Australia}

Recent theories on the pathophysiology of Parkinson's disease (PD) emphasize early brainstem involvement. Furthermore various respiratory function abnormalities have been reported without consistent pattern. We sought to study the effects of idiopathic PD on respiratory function and ventilatory response to hypercapnoea and hypoxia.

Methods Patients with a diagnosis of PD but no known respiratory disease were recruited. Subjects underwent lung function testing including respiratory muscle strength, ventilatory response to hypercapnoea (with central respiratory drive $\left.\left(\mathrm{P}_{100}\right)\right)$ and a hypoxic simulation $\left(\mathrm{FIO}_{2} 15 \%\right)$. PD severity was assessed by standardized scales; the Hoehn \& Yahr ( $\mathrm{H} \& \mathrm{Y})$, and unified Parkinson's disease rating scale (UPDRS). A linear correlation was used to identify associations between respiratory function, ventilatory responses and disease severity.

Results 19 subjects (17 males) were studied (mean (SD) age 66 (8) years). The UPDRS (motor examination) was 19 (8) and H \& Y 2.5 (0.5). Lung function was normal in $16(84 \%)$ ( 1 mild obstruction, 2 mild restriction). Maximum inspiratory pressure was reduced in $8(42 \%)\left(64(28) \mathrm{cmH}_{2} \mathrm{O}\right)$, and maximum expiratory pressure was reduced in $9(47 \%)\left(99(54) \mathrm{cm} \mathrm{H}_{2} \mathrm{O}\right)$. The response to hypoxia was normal. Four subjects were unable to complete the hypercapnoeic challenge. $7 / 15(47 \%)$ had a reduced ventilatory response to hypercapnoea which was predominately attributable to a reduced central respiratory drive, as $11 / 15(73 \%)$ had an abnormal $P_{100}$ response. No associations were observed between disease severity markers, respiratory function or ventilatory responses.

Conclusion Subjects with mild to moderate PD had predominantly normal respiratory function. However, nearly half had reduced respiratory muscle strength and a dulled ventilatory response to hypercapnoea due to a reduced central respiratory drive. These observations may have implications for management strategies for PD patients with respiratory symptoms or infections. Conflict of Interest No.

\section{IS THE CPAP IMPLEMENT STUDY A DINOSAUR?}

\section{S KAUL, D LANGTON, G BRAUN, D PANETTIERI, V LEBLANC} Department Thoracic Medicine/Sleep, Frankston Hospital, Vic

Once obstructive sleep apnoea (OSA) is diagnosed, a CPAP implementation sleep study is traditionally performed to determine the pressure required to control the upper airway. However, since modern CPAP machines store sophisticated control data we reasoned it may equally be possible to commence CPAP via a 'best guess' iterative approach without compromising OSA control or compliance.

Aim To compare the outcomes at 3 months of patients commencing CPAP after best guess with those commencing CPAP after a CPAP implementation sleep study.

Methods We retrospectively reviewed the records of all patients referred by respiratory physicians to our CPAP clinic between March 2008 and March 2010, and the two methods of starting CPAP were compared. Data collected included age, sex, BMI, respiratory disturbance index (RDI), CPAP pressure commenced, final pressure at 3 months, CPAP usage data and CPAP clinic contacts.

Results 287 patients were identified, aged $59 \pm 13$ years, $66 \%$ male, BMI $36.4 \pm 8.1$, with severe OSA, RDI $44 \pm 25.183$ commenced CPAP via best guess and 107 after a CPAP sleep study. The starting pressures in both groups were similar, $9.6 \pm 3.0$ versus $8.8 \pm 1.1 \mathrm{cmH} 2 \mathrm{O}$. In those patients continuing to use CPAP at 3 months, there were no differences between the groups for final pressure, numbers of patients changing pressure, control of OSA with CPAP, and hours CPAP used per day. In the best guess group however, significantly more patients were continuing to use CPAP at 3 months, $87 \%$ versus $71 \%(p=0.004)$.

Conclusion This study demonstrates that it may no longer be necessary to perform CPAP implementation sleep studies routinely and this will save hospital bed days.

Conflict of Interest Nil. 


\section{A HYPOXIC INHALATION TEST IS UNNECESSARY IF OXYGEN SATURATION AFTER MODERATE EXERCISE IS $95 \%$ OR HIGHER}

IT LING, B SINGH, AL JAMES, DR HILLMAN

Department of Pulmonary Physiology, Sir Charles Gairdner Hospital, WA, Australia

Background The hypoxic inhalation test (HIT) accurately predicts hypoxaemia during air travel but is only available in specialized lung function laboratories.

Aim To determine whether an abnormal HIT can be predicted by simple and widely available measures of respiratory function.

Methods Retrospective analysis of all patients who had a HIT between Jan 2005 and Apr 2010 and measurements of ventilatory capacity and single breath transfer factor (DLCO) within 6 months of HIT. Prior to HIT, oxygen saturation $\left(\mathrm{SpO}_{2}\right)$ was measured after moderate exercise (stepping for 2 minutes). The relationship between $\mathrm{HIT} \mathrm{SpO}$ and resting room air $\mathrm{SpO}_{2}$, post-exercise room air $\mathrm{SpO}_{2}$, DLCO and ventilatory capacity were examined. Mixed effects linear regression was performed and significant variables $(5 \%$ level) were retained to for the final model.

Results 137 patients were identified: 70 had chronic obstructive pulmonary disease (COPD), 43 had interstitial lung disease (ILD) and 20 had extra-pulmonary restriction (EPR). Post-exercise room air $\mathrm{SpO}_{2}$ correlated best with HIT SpO ${ }_{2}$ overall $(r=0.69, p<0.01)$ and in all disease subgroups except COPD (Resting $\mathrm{SpO}_{2} \mathrm{r}=0.72$ vs. Post-exercise $\mathrm{SpO}_{2} \mathrm{r}=0.70$, both $\mathrm{p}<0.01$ ). All patients who had a post-exercise $\mathrm{SpO}_{2}$ of $\geq 95 \%$ also had a HIT $\mathrm{SpO}_{2}$ of $>85 \%(\mathrm{n}=56(41 \%))$. Linear regression identified $\mathrm{FVC} \%$ predicted, resting $\mathrm{SpO}_{2}$, and post-exercise $\mathrm{SpO}_{2}$ as independent predictors of HIT $\mathrm{SpO}_{2}$. HIT $\mathrm{SpO}_{2} \leq 85 \%$ was associated with DLCO $<65 \%$ predicted and $<50 \%$ predicted in COPD and ILD, respectively, and with FVC $<40 \%$ predicted in EPR.

Conclusions In patients with chronic respiratory impairment, ventilatory capacity correlates poorly with HIT $\mathrm{SpO}_{2}$. Post-exercise $\mathrm{SpO}_{2}<95 \%$ may be used to select patients who require assessment by HIT. HIT $\mathrm{SpO}_{2}$ can be estimated from $\mathrm{FVC} \%$ predicted, resting $\mathrm{SpO}_{2}$, and post-exercise $\mathrm{SpO}_{2}$ using a linear regression model.

Support and Conflict of Interest Nil.

\section{CHARACTERISTIC PATTERNS OF OXYGEN DESATURATION IN SLEEP DISORDERED BREATHING}

R NANKERVIS ${ }^{1,2}$, C ZAPPALA ${ }^{1,2}$, S MORRISON $^{1,2}$

${ }^{1}$ Rivercity Sleep Laboratories, Brisbane, Australia, and ${ }^{2}$ Department of

Thoracic Medicine, Royal Brisbane and Women's Hospital, Brisbane, Australia

Aim We hypothesized that different types of sleep disordered breathing may be associated with specific changes in the oxygen saturation (SpO2) trace. This study aims to characterize the $\mathrm{SpO} 2$ trace in obstructive sleep apnoea, central sleep apnoea and Cheyne-Stokes respiration.

Methods Overnight polysomnographic studies were selected from the sleep database from January to March 2010 that demonstrated either an obstructive or central respiratory disturbance index of greater than 30 events per hour. Cheyne-Stokes respiration cases were selected from studies undertaken from October 2009 to June 2010. The SpO2 trace in each study was analysed to determine the gradient of desaturation (DS) and resaturation (RS) and the magnitude of the nadir in the $\mathrm{SpO} 2$ for each event. We used the ratio of DS to RS as a primary outcome variable. DS/RS was then averaged for each patient. One way ANOVA was used to determine the difference in mean DS/ RS ratio between respiratory event types.

Results 1880 obstructive respiratory events were analysed from 10 patients and the mean $\mathrm{DS} / \mathrm{RS}$ ratio was $0.46(\mathrm{SE}=0.03) .1428$ central respiratory events were analysed from 8 patients and the mean DS/RS ratio was 0.61 $(S E=0.03) .1032$ Cheyne-Stokes respiration events were analysed from 8 patients the mean $\mathrm{DS} / \mathrm{RS}$ ratio was $0.81(\mathrm{SE}=0.04)$ One way ANOVA analysis of the mean DS/RS ratios revealed a significant difference in the three patterns of sleep disordered breathing $(p=0.001)$. Cheyne-Stokes events had a significantly higher mean DS/RS ratio than either central $(p=0.002)$ or obstructive $(p<0001)$ sleep apnoeic events. Central events had a higher mean DS/RS ratio than obstructive events $(p=0.023)$

Conclusions There appears to be a significant difference in the SpO2 DS/ RS ratios in the different types of sleep-disordered breathing. Further prospective trials are underway to determine if this finding has diagnostic utility.

Supported by Nil.

Nomination Nil.

Conflict of Interest No.

\section{TP-151}

\section{HIGH PREVALENCE OF DEPRESSION AMONGST SNORERS}

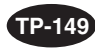

A CASE SERIES OF MORBIDLY OBESE PATIENTS ADMITTED TO INTENSIVE CARE WITH RESPIRATORY FAILURE DUE TO PREVIOUSLY UNDIAGNOSED OBESITY HYPOVENTILATION OR OVERLAP SYNDROME

MP FANNING ${ }^{1}, \mathrm{~K}^{\text {TRAN }}{ }^{2,3}$

${ }^{1}$ Department of Medicine, Logan Hospital, Qld, Australia, ${ }^{2}$ Department of Medicine, Logan Hospital, QId, Australia, and ${ }^{3}$ Sleep Disorders Centre, Princess Alexandra Hospital, Qld, Australia

Aim To describe a case series of morbidly obese patients, who presented to intensive care (ICU) with hypercapnoeic respiratory failure, due to previously undiagnosed obesity hypoventilation syndrome or overlap syndrome. To our knowledge, there is only one other similar case series previously described. Method We collected data on morbidly obese patients who were admitted to the Intensive Care Unit at Logan Hospital in acute on chronic hypercapnoeic respiratory failure and who were later diagnosed with accompanying sleep disordered breathing between 2003 and 2009.

Results Eighteen patients were identified within the study period. The average age was 53 and the average body mass index was 53 . Eight had a smoking history and six had evidence of airflow limitation with two being in the very severe category ( $<35 \%$ predicted). The average apache II score was 20 . Six required intubation and the rest were managed with non-invasive ventilation in ICU. The average length of stay in ICU was 9.28 days. Polysomnographic data will be described.

Conclusions Obesity hypoventilation as a cause of respiratory failure is likely to increase in frequency as the incidence of obesity increases. Increased awareness by the lay public, as well as clinical suspicion and recognition of the condition by all clinicians at an earlier stage, is likely to prevent progression to the point of needing intensive care. It is hoped that this case series may provide a springboard for further study into why these patients presented at such a late stage of their disease process.

Supported by None.

Conflict of Interest None.

Journal Compilation (C) 2011 Asian Pacific Society of Respirology
N DOUGLAS, A YOUNG, T ROEBUCK, S HO, BR MILLER, K KEE, MT NAUGHTON

Dept of Allergy, Immunology \& Respiratory Medicine, Alfred Hospital and Monash University Melbourne, Australia

Rationale Depression, a disorder of disturbed sleep and fatigue, is the commonest cause of disability affecting 150 million with an estimated loss of 1 million pa. Only $10-25 \%$ are recognized and treated. Estimates in sleep apnoea (SA) vary from 7-63\%. Treatment of SA improves depression, whereas if left untreated the risk of developing depression increases 3 -fold. Although SA and sleepiness often co-exist, the commonest cause of sleepiness in a general community is depression, with SA being the 7th most common cause. In order to assist recognition of depression in a snoring population attending a sleep clinic, we introduced a simple two question 'Beyond Blue Questionnaire(BBQ)' into our routine assessment.

Aims To (1) determine the prevalence of depression in snorers using validated questionnaire and/or doctor diagnosis, (2) compare the accuracy of $\mathrm{BBQ}$, and (3) to correlate depression severity with sleep parameters.

Methods Consecutive snorers for investigation underwent clinical assessment by a sleep physician then completed the 14 question Hospital Anxiety and Depression Score (HADS) and BBQ questionnaires in addition to ESS and NYHA plus underwent a sleep study.

Results 142 subjects were approached of whom 133 completed all questionnaires (mean age 52 years, BMI $33 \mathrm{~kg} / \mathrm{m} 2,65 \%$ male). $43 \%$ had either a doctor diagnosis or positive HADS ( $\geq 16)$. HADS and BBQ correlated significantly $(r$ $=0.736, p<0.001$ ). Importantly 18 and $27 \%$ of subjects with no prior doctor diagnosis of depression had positive HADS or BBQ questionnaires respectively. Sensitivity and specificity of BBQ vs doctor diagnosis or +ve HADS were of depression (HADS and BBQ) correlated with NYHA and ESS, minimum $\mathrm{SpO} 2$ but not $\mathrm{AHI}$ or sleep efficiency. nized. The simple 2 question BBQ assists in diagnosis with reasonable sensitivity and specificity.

Conflict of Interest Nil.

Support Nil. 74 and $76 \%$ respectively and PPV and NPV 70 and $79 \%$ respectively. Severity

Conclusion In snorers, depression is common \& frequently under-recog- 


\section{OUTCOMES OF PATIENTS ADMITTED FOR WEANING AT AN AUSTRALIAN NON-ICU VENTILATION WEANING UNIT}

L HANNAN $^{1}$, S TAN ${ }^{1}$, E BAKER ${ }^{1}$, K HOPKINSON $^{1}$, S TAN ${ }^{1}$, D BERLOWITZ ${ }^{1,2}$, C MCDONALD $^{1,2}$, M HOWARD $^{1,2}$

${ }^{1}$ Austin Health, Department of Respiratory and Sleep Medicine, Heidelberg, Victoria, and ${ }^{2}$ Institute for Breathing and Sleep, Austin Health, Heidelberg, Victoria

Introduction The Ventilation Weaning Unit (VWU) was established at Austin Health in 2002 as a state-wide quaternary referral centre for patients with delayed or failed weaning from invasive mechanical ventilation (IMV) in intensive care units (ICU). Outcomes for a non-ICU weaning unit have not been reported previously in Australia.

Methods Retrospective cohort study of patients admitted to the VWU from March 2002 until January 2008. All patients admitted to the unit with the intention of liberation from IMV were included in the study.

Results A total of 78 patients were admitted to the VWU after a median of 27 days of IMV. Weaning success (ventilator free or nocturnal non-invasive ventilation only) was achieved in $78.2 \%(n=61)$ of patients. Inpatient mortality was $10.2 \%(n=8)$ and serious complications were infrequent. Progressive neuromuscular disease (OR 0.10 ) and sepsis (OR 0.09) were predictive of a lower likelihood of weaning success at the time of discharge from the VWU. Overall survival at 12 months following discharge from the VWU was $66.7 \%$ $(n=52)$ with most survivors residing in the community.

Conclusions Selected patients with weaning delay or failure can achieve high rates of successful weaning from IMV in a non-ICU ventilation weaning unit with acceptable complication and mortality rates.

Conflict of Interest None.

\section{BETWEEN INTERPRETER DIFFERENCES IN MULTIPLE NITROGEN BREATH WASHOUT ANALYSIS OF VENTILATION DISTRIBUTION}

\author{
G PALNITKAR ${ }^{1}$, R SCHOEFFEL ${ }^{1}$, S TIMMINS ${ }^{1,2,3,4}$, S MAHADEV ${ }^{1,2,3}$, \\ C SALOME ${ }^{2,3,4}$, G KING $^{1,2,3,4}$ \\ ${ }^{1}$ Department of Respiratory Medicine, Royal North Shore Hospital, ${ }^{2} T$ The \\ Woolcock Institute of Medical Research, ${ }^{3}$ The University of Sydney, ${ }^{4}$ The \\ CRC for Asthma and Airways
}

Background Indices of ventilation distribution in diffusion $\left(\mathrm{S}_{\text {acin }}\right)$ and convection $\left(\mathrm{S}_{\text {cond }}\right)$ dependent airways derived from multiple breath nitrogen washout (MBNW) may vary between interpreters because of differences in calculation of phase III slopes ( $\Delta$ phase III). $_{\text {. }}$.

Aims To compare $S_{\text {cond }}$ and $S_{\text {acin }}$ results of 3 interpreters from a single MBNW in 10 COPD subjects.

Methods 10 subjects with COPD underwent MBNW. Three washouts were analysed independently by 1 experienced and 2 novice interpreters using custom software for automated breath identification. $\Delta$ phase $_{\| \mid}$was fitted automatically by least squares fit between 2 predetermined points, and then adjusted manually. $\mathrm{S}_{\text {cond }}$ was the linear slope of $\Delta$ phase ${ }_{\| 1}$ plotted against lung turnover (cumulative expired volume/FRC), between turnovers 1.5-6. $S_{\text {acin }}$ was the $\Delta$ phase $_{\text {III }}$ of the first breath minus the $S_{\text {cond }}$ component. Differences expressed as ICC and COV, were examined by repeated measures ANOVA. Results Mean \pm SD age was $71 \pm 10$ years. FEV was $62 \pm 15 \%$ predicted. $\mathrm{S}_{\text {cond }}$ was greater while $\mathrm{S}_{\text {acin }}$ was lower from the experienced

\begin{tabular}{llll}
\hline Interpreter & \multicolumn{1}{c}{ Scond L } & \multicolumn{1}{c}{ Sacin $\mathrm{L}^{-1}$} & \multicolumn{1}{c}{ FRC (L) } \\
\hline Experienced & $0.051 \pm 0.030$ & $0.545 \pm 0.218$ & $3.15 \pm 0.85$ \\
Novice1 & $0.036 \pm 0.019$ & $0.6251 \pm 0.230$ & $3.22 \pm 0.87$ \\
Novice2 & $0.036 \pm 0.026$ & $0.616 \pm 0.231$ & $3.12 \pm 0.86$ \\
COV & 0.62 & 0.37 & 0.26 \\
ICC & 0.10 & 0.40 & 0.92 \\
\hline
\end{tabular}

interpreter ( $p=0.01$ and $p<0.0001$, respectively) but not different between novice interpreters. FRC was no different between all three.

Conclusion Differences in MBN analysis may occur between interpreters, which may be dependent on prior analysis experience. It is necessary to standardize MBNW analysis approaches.

Supported by CRC for Asthma and Airways, UPA and ALF Postgraduate Scholarship.

Nomination Nil.

Conflict of Interest No.

\section{THE RELATIONSHIP BETWEEN TRANSFER FACTOR AND AIRWAY REACTIVITY IN ASTHMA}

RL SHRESTHA ${ }^{1}$, IT LING ${ }^{2}$, W NOFFSINGER ${ }^{2}$, B SINGH ${ }^{2}$

Departments of Respiratory Medicine 1 \& Pulmonary Physiology?,

Sir Charles Gairdner Hospital, WA, Australia

Background and Aim The single breath carbon monoxide transfer factor (DLCO) may be normal or increased in patients with asthma. The aim of this study was to examine the relationship between airway reactivity and DLCO in patients with asthma.

Methods Retrospective analysis of all patients who had a methacholine bronchial provocation test (BPT-M) and DLCO on the same day between 2005 and 2010. We examined the relationships between DLCO, a positive BPT-M ( $F E V_{1}$ decreased by $>20 \%$ ), the magnitude of bronchial hyper-responsiveness (PD20), BMI and smoking status.

Results We identified 283 patients who had a BPT-M and DLCO on the same day. The BPT-M was positive in $78(27.5 \%)$ and, of these, 41 patients $(52.5 \%)$ had a clinical diagnosis of asthma. DLCO was elevated $(>120 \%$ predicted, i.e. above upper limit of normal) in $15(6 \%)$ patients. The age and BMI of those with a positive BPT-M were (mean \pm SD) 51 years \pm 16 and $29 \mathrm{~kg} / \mathrm{m}^{2} \pm 6$, respectively. Compared to patients without a clinical diagnosis of asthma, those with a clinical diagnosis of asthma were more likely to have an elevated DLCO corrected for alveolar volume (odds ratio 6.0). There was no relationship between DLCO and PD20. A high DLCO in this subset of asthmatics was associated with the absence of current smoking.

Conclusions An increased DLCO was associated with a clinical diagnosis of asthma but not with objective measures of airway reactivity.

Conflict of Interest Nil. 


\section{B-BLOCKER USAGE BY PATIENTS REFERRED FOR LUNG} FUNCTION TESTING: AN OBSERVATIONAL STUDY

\section{S PRADEEPAN, R TIMO, A THORNTON, S JOHNSTON, H GREVILLE} Department of Thoracic Medicine, Royal Adelaide Hospital. South Australia

Introduction $\beta$-blockers may cause bronchoconstriction and mask the effect of $\beta_{2}$-adrenergic agonists. This has implications for the interpretation of routine diagnostic spirometry and bronchodilator response. This study examined this issue in a routine lung function laboratory, and whether it applied to both cardio-selective $(\mathrm{C})$ and non-selective (NC) preparations.

Method All patients attending the Lung Function Laboratory, Royal Adelaide Hospital over a 2-month period were asked whether they were currently taking a $\beta$-blocker and to identify the drug. Spirometry results were analysed to assess airflow obstruction and reversibility.

Results 407 patients completed the survey and 60 patients (14\%) were taking $\beta_{2}$-blockers. The table shows the results of the 352 patients who could be assessed for reversibility in spirometry. Of the 48 patients in this group 40 patients (83\%) were taking (C) and $8(17 \%)(\mathrm{NC})$ agents. Fifty-three patients were unsure whether they were taking a $\beta_{2}$-blocker. No significant differences were found in the percentage of patients with airflow obstruction or reversibility between the groups.

\begin{tabular}{|c|c|c|c|c|}
\hline & \multicolumn{2}{|c|}{$\beta$ blocker } & \multirow[b]{2}{*}{ Unsure } & \multirow[b]{2}{*}{ No $\beta$ blocker } \\
\hline & $\mathrm{C}$ & $\mathrm{NC}$ & & \\
\hline Patients & 40 & 8 & 53 & 251 \\
\hline $\mathrm{AO}$ & $9(23 \%)$ & $3(38 \%)$ & $16(30 \%)$ & 92 (37\%) \\
\hline REV & $5(13 \%)$ & $1(13 \%)$ & $7(13 \%)$ & $39(16 \%)$ \\
\hline $\mathrm{AO}+\mathrm{REV}$ & $4(10 \%)$ & 0 & $3(6 \%)$ & 31 (12\%) \\
\hline
\end{tabular}

Data are expressed as number of patients and percentage in brackets $A O$ : Airflow Obstruction; REV: Reversible spirometry based on ATS ERS; C: Cardioselective; NC: Non-cardioselective

Conclusion A significant proportion of patients presenting for lung function testing are on $\beta$-blockers. Of interest is that 53 patients were not certain whether they were taking a $\beta 2$-blocker. The patient numbers in the $\beta 2$-blocker groups are small but no obvious pattern of airflow obstruction or reversibility emerged. There is still an onus on the referring physician to provide a current medications list where appropriate.

Conflicts of Interest Nil.

Nomination Janet Elder International travel Award.

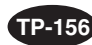

\section{PREDICTORS OF DIFFUSION AND CONVECTION DEPENDENT}

\section{VENTILATION HETEROGENEITY IN COPD}

KO TONGA ${ }^{1}$, RE SCHOEFFEL ${ }^{1}$, SC TIMMINS ${ }^{1,2,3,4}$, S MAHADEV ${ }^{1,2,3}$, CM SALOME ${ }^{2,3,4}$, GG KING KI,2,3,4 $^{1,}$

${ }^{1}$ Department of Respiratory Medicine, Royal North Shore Hospital, St Leonards, NSW, ${ }^{2}$ Woolcock Institute of Medical Research, Glebe NSW, ${ }^{3}$ Northern Clinical School, University of Sydney, NSW, and ${ }^{4}$ CRC for Asthma and Airways, Glebe, NSW

Introduction Multiple Breath Nitrogen Washout (MBNW) measures ventilation heterogeneity in diffusion dependent (Sacin) and convection dependent airways (Scond). Single Breath Nitrogen Washout (SBNW) provides a noncompartmentalized measure of ventilation distribution $\left(\triangle \mathrm{Phase}_{\mathrm{II}}\right)$, and airway closure (closing volume CV and closing capacity $\mathrm{CC}$ ). Both are considered measures of small airway function but the nature of their relationship is unknown.

Aim To determine the relationships between Sacin, Scond, $\Delta$ Phase $_{\text {III }}$ and CV.

Methods 32 patients with COPD and 10 normal subjects underwent spirometry, plethysmograph lung volumes, CV and CC from SBNW, and Sacin and Scond from MBNW to measure ventilation heterogeneity. Predictors of Sacin and Scond were examined by multiple regression analyses.

Results COPD patients were mean \pm SD $69 \pm 8$ years, had $52 \pm 21$ pack/ years smoking history, $\mathrm{FEV}_{1} 59 \pm 17 \%$ predicted, $\mathrm{FEV}_{1} / \mathrm{VC} 53 \pm 12 \%, \Delta$ Phase $_{\text {III }}$ $8.81 \pm 4.57 \% / \mathrm{L}, \mathrm{CV} 18 \pm 8 \%$ of VC, CC $=62 \pm 8 \%$ of TLC, Sacin $0.534 \pm$ $0.171 \mathrm{~L}^{-1}$, Scond $0.054 \pm 0.023 \mathrm{~L}^{-1}$ (normal Sacin $<0.130 \mathrm{~L}^{-1}$, Scond $<0.037 \mathrm{~L}^{-1}$ ). The normal subjects were $31 \pm 7$ years and had normal spirometry, Sacin $0.120 \pm 0.047 \mathrm{~L}^{-1}$, Scond $0.016 \pm 0.01 \mathrm{~L}^{-1}$. Sacin was predicted by $\Delta$ Phase $_{\| I}$, $\mathrm{CV}$ and RV/TLC. In multi-variate analysis, Sacin was predicted by all three parameters (model $r^{2}=0.77, p<0.0001$ ).

Conclusion Sacin is measure of small airways disease that reflects both ventilation heterogeneity and airway closure as measured by SBNW in COPD. There does not appear to be a relationship with Scond.

Support S Mahadev- Asthma Foundation NSW stipend.

Conflict of Interest No.

Journal Compilation @ 2011 Asian Pacific Society of Respirology

\section{SPIROMETRY ALONE MISSES SOME ABNORMAL LUNG FUNCTION PHENOTYPES IN ADULTS}

JL PERRET ${ }^{1,2,3}$, SC DHARMAGE ${ }^{1}$, MC MATHESON $^{1}$, J MARRONE ${ }^{4}$, D MÉSZÁROS ${ }^{4}$, CF MCDONALD ${ }^{2,3}$, PS THOMAS $^{5}$, D JOHNS ${ }^{4}$, MJ ABRAMSON ${ }^{6}$, EH WALTERS ${ }^{4}$

${ }^{1}$ MEGA Epidemiology, Uni of Melbourne, Vic., ${ }^{2}$ Austin Hospital, Melbourne, VIC; ${ }^{3}$ Institute of Breathing and Sleep, Melbourne, Vic., ${ }^{4}$ Menzies Research Institute, Tasmania, and ${ }^{5}$ Faculty of Medicine, UNSW; ${ }^{6}$ Monash University, Melbourne, Vic

Aim To examine patterns of adult lung function in terms of airflow obstruction, hyperinflation and/or reduced diffusing capacity $\left(D_{L} C O\right)$. This can then be related to the life-time history of risk factors such as smoking, asthma and infections.

Methods Using the population-based Tasmanian Longitudinal Health Study (TAHS) cohort followed since 1968, an asthma-enriched sub-sample was selected consisting of $50 \%$ ever with asthma, of whom half reported current asthma.

Measurement of spirometry, $\mathrm{D}_{\mathrm{L}} \mathrm{CO}$ (uncorrected for haemoglobin) and lung volumes was performed, then lung function data were analysed using the mean predicted values. Airflow obstruction was defined as post-bronchodilator $\mathrm{FEV}_{1} / \mathrm{FVC}$ (post-b.d. FER) $<0.7$, hyperinflation as total lung capacity (TLC) $>120 \%$ predicted, and reduced $D_{1} \mathrm{CO}$ as $<80 \%$ predicted.

Results The average age of participants was 44.8 years (range 43-47), with full lung function data available for $n=1197.9 .4 \%(n=112)$ had $F E V_{1} / F V C$ post-b.d. $<0.7$. Lung function was within normal limits in $61 \%(n=732)$.

\begin{tabular}{lcccccccc}
\hline & 732 & 138 & 194 & 21 & 57 & 13 & 29 & 13 \\
Subject $\mathrm{n}=(\%)$ & $(61)$ & $(11.5)$ & $(16.2)$ & $(1.8)$ & $(4.8)$ & $(1.1)$ & $(2.4)$ & $(1.1)$ \\
\hline Post b.d. FER $<0.7$ & - & - & - & - & + & + & + & + \\
TLC $>120 \%$ pred. & - & - & + & + & - & - & + & + \\
$\mathrm{D}_{\mathrm{L} C O}<80 \%$ pred, & - & + & - & + & - & + & - & + \\
\hline
\end{tabular}

Conclusion Isolated elevated TLC or reduced $D_{L} C O$ are commonly seen in middle aged adults, and these individuals would have been classified as having 'normal lung function' if assessed with spirometry alone.

Grant Support NHMRC, Australian Postgraduate Association. Conflict of Interest No. 
FEMALES HAVE LOWER DIFFUSING CAPACITY $\left(D_{L} C O\right)$ THAN MALES, WITH AND WITHOUT CORRECTION FOR ALVEOLAR VOLUME $\left(\mathrm{V}_{\mathrm{A}}\right)$

\author{
JL PERRET ${ }^{1,2}$, EH WALTERS ${ }^{3}$, MC MATHESON ${ }^{1}$, J MARRONE $^{3}$, \\ D MÉSZÁROS ${ }^{3}$, CF MCDONALD ${ }^{2}$, PS THOMAS ${ }^{4}$, MJ ABRAMSON ${ }^{5}$, \\ D JOHNS ${ }^{3}$, SC DHARMAGE ${ }^{1}$ \\ ${ }^{1} M E G A$ Epidemiology, Uni of Melbourne, ${ }^{2}$ Austin Hospital, Melbourne, \\ ${ }^{3}$ Menzies Research Institute, Tasmania, ${ }^{4}$ Medicine, UNSW, ${ }^{5}$ Monash \\ University, Melbourne
}

Aim To examine the gender-specific differences in adult spirometry, $\mathrm{D}_{\mathrm{L}} \mathrm{CO}$ and lung volumes, with a view to relating them to life-time respiratory risk factors. Methods Using the population-based Tasmanian Longitudinal Health Study (TAHS) followed since 1968, an asthma-enriched sub-sample was selected consisting of $50 \%$ ever with asthma, of whom half reported current asthma. Measurement of spirometry, $D_{L} C O$ (corrected for haemoglobin) and lung volumes were performed. Data were analysed using the statistical upper and lower limits of normal of reference equations by NHANES III, Roca et al and Quanjer et al.

Results Of the 1365 Caucasian adults (665 females), $80 \%$ completed all tests.

\begin{tabular}{lll}
\hline Abnormality & Males (n (\%) 95\% Cl) & Females (n (\%) 95\% Cl) \\
\hline Low post-b.d. FEV $_{1}$ & $70(10)(7.8-12.2)$ & $65(9.8)(7.5-12.0)$ \\
Low post-b.d. FEV $/$ FVC & $67(9.6)(7.4-11.8)$ & $68(10)(7.7-12.3)$ \\
Reduced $D_{L}$ CO (corr) & $83(14.1)(11.3-16.9)$ & $114(19.7)(16.4-22.9)$ \\
Reduced $D_{L} C O / V_{A}$ (corr) & $81(13.8)(11.0-16.6)$ & $223(38.5)(34.5-42.5)$ \\
Elevated TLC & $81(13)(10.3-15.5)$ & $77(13)(10.2-15.6)$ \\
\hline
\end{tabular}

Mean age 44.8 years (range 43-47). Elevated rates of airflow obstruction and hyperinflation were seen. Significantly higher proportions of females than males had reduced $D_{L} C O$ and $D_{L} C O / V_{A}(p<0.05)$. Only $3.2 \%(n=18)$ of females had a low $D_{L} C O$ with low $F E V_{1} / F V C$ ratio, and $1.7 \%(n=23)$ had a reduced TLC overall. There were no significant gender differences in $V_{A}, T L C$, or ever and current active smoking. Males and females averaged over $15 \mathrm{~kg}$ more than the Mediterranean adults described by Roca et al., however weight is not relevant to $D_{1} C O$ in males.

Conclusion $\mathrm{A}$ higher percentage of middle aged females have a reduced $\mathrm{D}_{\mathrm{L}} \mathrm{CO}$ and/or $\mathrm{D}_{\mathrm{L}} \mathrm{CO} / \mathrm{N}_{\mathrm{A}}$, compared to males, with an increased rate overall. Grant Support NHMRC, Australian Postgraduate Association.

Conflict of Interest No.

\section{AIRWAY DISTENSIBILITY IS NOT ASSOCIATED WITH AIRWAY} RE-NARROWING FOLLOWING DEEP INSPIRATION

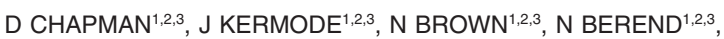
G KING $^{1,2,3,4}$, C SALOME ${ }^{1,2,3}$

${ }^{1}$ Woolcock Institute of Medical Research, Sydney, NSW, ${ }^{2}$ University of Sydney, NSW, ${ }^{3}$ CRC for Asthma, Sydney NSW, and ${ }^{4}$ Department of

Respiratory Medicine, Royal North Shore Hospital, NSW

Background During bronchoconstriction, a deep inspiration (DI) dilates the airways, which then re-narrow once tidal breathing is resumed. Re-narrowing occurs faster in asthmatic subjects and may be due reduced airway distensibility.

Aim To determine the association between baseline airway distensibility and the rate of re-narrowing after DI.

Methods Eleven asthmatic and five non-asthmatic subjects had baseline airway distensibility measured by forced oscillation technique (FOT). After methacholine challenge, respiratory system resistance (Rrs) was measured during 1 min of tidal breathing, followed by DI to total lung capacity (TLC) and passive return to normal tidal breathing. Dilatation was measured as the decrease in Rrs between end tidal inspiration and TLC, and re-narrowing as end-expiratory Rrs immediately after $\mathrm{DI}$, as per cent Rrs at end-tidal expiration before the DI. Distensibility is presented as geometric mean $\pm 95 \% \mathrm{Cl}$ and re-narrowing as mean $\pm 95 \% \mathrm{Cl}$.

Results Airway distensibility was reduced in asthmatic compared to healthy subjects $\left(0.17 \mathrm{~s}^{-1} . \mathrm{cmH}_{2} \mathrm{O}^{-1}(0.11-0.23)\right.$ vs. $0.35 \mathrm{~s}^{-1} . \mathrm{cmH}_{2} \mathrm{O}^{-1}(0.18-0.66), \mathrm{p}=$ $0.04)$. Dilatation did not differ between groups $(p=0.79)$ but re-narrowing was increased in asthmatic compared to healthy subjects $(129 \pm 38 \%$ vs. $53 \pm 13 \%$, $p=0.02)$. Airway distensibility did not correlate with airway re-narrowing $\left(r_{s}=\right.$ $-0.07, p=0.80$ )

Conclusion The increased re-narrowing after $\mathrm{DI}$ in asthmatic subjects is not due to reduced baseline airway distensibility and may be due to increased shortening velocity of airway smooth muscle or reduced elastic recoil.

Supported by The NHMRC and the CRC for Asthma and Airways.

Nomination Nil.

Conflict of Interest No.

\section{PHYSIOTHERAPY SIG: POSTER SESSION}

POSTER WITHDRAWN
MEASUREMENT PROPERTIES OF TWO ACCELEROMETERS IN PEOPLE WITH COPD: EFFECT OF WALK SPEED AND 4-WHEELED WALKER USE

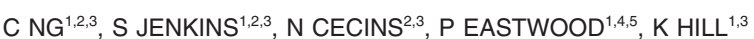
${ }^{1}$ Physiotherapy, Curtin University, Bentley, WA, ${ }^{2}$ Physiotherapy Department \& ${ }^{4}$ Pulmonary Physiology, Sir Charles Gairdner Hospital, Nedlands, WA, ${ }^{3}$ Lung Institute of Western Australia, Nedlands, WA, ${ }^{5}$ School of Anatomy and Human Biology, University of Western Australia, Nedlands, WA

Aim To evaluate the measurement properties of two accelerometers: the ActivPAL and the StepWatch Activity Monitor (SAM) in people with COPD.

Methods The ActivPAL and SAM were attached to the anterior right midthigh and the right ankle, respectively (as per device recommendations). Each participant performed 4 walking tasks; 2 at a self-selected slow speed and 2 at a self-selected normal speed. At each speed, one walk was performed with a 4-wheeled walker (WW) and the other without.

Results 20 participants aged 73 (9) years $\left(\mathrm{FEV}_{1}=35(13) \%\right.$ pred; 8 males) completed the study. The slow and normal speeds were $34(7) \mathrm{m} \cdot \mathrm{min}^{-1}$ and 46 (10) $\mathrm{m} \cdot \mathrm{min}^{-1}$, respectively. Agreement between steps recorded by the SAM with steps counted during observation did not differ with speed or WW use ( $p$ $=0.63$ ). The mean difference was 2 steps $\cdot \mathrm{min}^{-1}$ and the limit of agreement (LOA) was 6 steps $\cdot \mathrm{min}^{-1}$. Agreement between steps recorded by the ActivPAL with steps counted was worse at slow speeds (mean difference 7 steps $\cdot \mathrm{min}^{-1}$ with LOA of 10 steps $\cdot \mathrm{min}^{-1}$ ) compared with normal speeds (mean difference 4 steps $\mathrm{min}^{-1}$ with LOA of 5 steps $\left.\cdot \mathrm{min}^{-1}\right)(p=0.03)$, but was not affected by WW use. Both accelerometers detected the small difference in walk speed irrespective of WW use $(p<0.001)$.

Conclusions Neither the accuracy nor responsiveness of either accelerometer was affected by WW use. In contrast to the ActivPAL, SAM was accurate at both speeds and therefore can be used to detect steps in people who walk very slowly during daily life.

Supported by Sir Charles Gairdner Hospital Research Fund.

Conflict of Interest No.

Journal Compilation @ 2011 Asian Pacific Society of Respirology 


\section{EXPLORING VARIATION IN THE CARE JOURNEYS OF PATIENTS WITH COPD}

AE HOLLAND ${ }^{1,2,3}$, NF TAYLOR ${ }^{2,4}$, A HUTCHINSON ${ }^{5}$, B MILLER ${ }^{1}$, F THIEN $^{4,6}$, SG LEGGAT ${ }^{2}$

${ }^{1}$ Alfred Health, Melbourne Vic., ${ }^{2}$ La Trobe University, Bundoora Vic. ${ }^{3}$ Institute for Breathing and Sleep, Heidelberg Vic., ${ }^{4}$ Eastern Health, Melbourne Vic., ${ }^{5}$ Northern Health, Epping Vic., and ${ }^{6}$ Monash University, Clayton Vic.

Aim To document the care and pathways patients with COPD travel at three metropolitan health services.

Methods Data were extracted from data sets for patients attending the emergency department of the three hospitals with a diagnosis of COPD ove 1 year. The three hospitals included a city-based tertiary/quaternary hospital and two smaller community hospitals. Analysis was completed on similarities and differences in admission and referral rates, average length of stay, and discharge destination, standardized by age, sex and mode of transport to the emergency department.

Results There were 2441 inpatient separations and 1182 emergency department presentations for patients with COPD. Discharge patterns related to the designated role of the hospital, with the community hospitals discharging 75 to $82 \%$ of patients directly home and the more specialized city hospital discharging $24 \%$ to other hospitals and $64 \%$ home. There were significant differences in the admission rates for category 3 and 4 patients among the hospitals We found unexplained variation in the acute average lengths of stay of 5.4 , 6.9 and 7.2 days.

Conclusions The analysis confirmed some expected patterns based on the type of hospital, but also identified unexplained variation that suggests that factors other than patient characteristics may be contributing to the variation in care pathways.

Supported by La Trobe University Faculty of Health Sciences.

Conflict of Interest No.

\section{TP-163}

DO ALL PATIENTS WITH COPD ATTENDING PULMONARY REHABILITATION NEED A PRACTICE 6-MINUTE WALK TEST (6MWT)?

S JENKINS ${ }^{1,2,3}$, N CECINS $^{1,2,3}$

${ }^{1}$ Physiotherapy, Sir Charles Gairdner Hospital, Perth, WA, ${ }^{2}$ School of Physiotherapy, Curtin University, Perth, WA, and ${ }^{3}$ Lung Institute of Western Australia, University of Western Australia, Perth, WA

In patients with COPD, two 6MWTs are recommended prior to commencing a pulmonary rehabilitation program (PRP) to allow for a learning effect.

Aim To determine the characteristics of patients with COPD in whom 6-minute walk distance (6MWD) did not increase on a second test.

Methods 245 patients (162 males) with stable COPD (aged 68, 37 to 86 years) naïve to the 6MWT performed two tests (30 minutes apart) prior to commencing a PRP. Patients were categorized according to their change in 6MWD with test repetition.

Results 6MWD was the same or decreased on the second test in 31 patients $(13 \%)$ (Table). In the remaining 214 patients (87\%), 6MWD increased by $44 \mathrm{~m}$ $(13 \%)(95 \% \mathrm{Cl} 40$ to $48 \mathrm{~m}, 12$ to $15 \%)$. Logistic regression analysis identified $\mathrm{FEV}_{1}(\mathrm{~L})$ as the only significant variable $(\mathrm{p}<0.01)$ that predicted the absence of a learning effect in 6MWD with test repetition.

Conclusions Some patients with severe COPD may not require a practice 6MWT to achieve their maximum performance at a PRP baseline assessment.

\begin{tabular}{|c|c|c|}
\hline & $\begin{array}{l}\text { No change/decrease } \\
\text { in 6MWD }(n=31)\end{array}$ & $\begin{array}{l}\text { Increase in 6MWD } \\
(n=214)\end{array}$ \\
\hline $\mathrm{FEV}_{1}(\mathrm{~L}, \%$ pred $)$ & $0.74(0.25)^{\star} 31(12)^{\star}$ & $1.10(0.50) 43(18)$ \\
\hline 6MWD Test 1 (m, \%pred) & 369 (112) $57(15)$ & $393(119) 60(18)$ \\
\hline Rested n (\%) & $17(55 \%)^{*}$ & $62(29 \%)$ \\
\hline Resting dyspnoea & $1.3(1.3)$ & $1.1(1.1)$ \\
\hline Peak dyspnoea & $5.2(1.8)$ & $4.5(1.7)$ \\
\hline Nadir $\mathrm{SpO}_{2}(\%)$ & $88(5)$ & $89(4)$ \\
\hline Peak heart rate (bpm) & $113(16)$ & $114(13)$ \\
\hline
\end{tabular}

$\mathrm{SpO}_{2}$, oxygen saturation; bpm, beats per minute. Mean (SD), ${ }^{*} \mathrm{p}<0.01$

Conflict of Interest No.
DEFINING THE RELATIONSHIP BETWEEN DAILY ENERGY EXPENDITURE, FIELD WALKING TESTS AND INTENSITY OF DAILY ACTIVITY IN COPD

$\mathrm{K} \mathrm{HILL}^{1,2,3}$, T DOLMAGE ${ }^{1}$, L WOON ${ }^{1}$, D COUTTS ${ }^{4}$, R GOLDSTEIN ${ }^{1,2}$, D BROOKS ${ }^{1,2}$

${ }^{1}$ Respirology, West Park Healthcare Centre, Ontario, Canada, ${ }^{2}$ Physical Therapy and Medicine, University of Toronto, Ontario, Canada,

${ }^{3}$ Physiotherapy and Curtin Health Innovation Research Institute, Curtin University, Bentley, WA, and ${ }^{4}$ Respiratory Medicine, Credit Valley Hospital, Mississauga, Canada

Aims To: (1) determine which tests of exercise capacity relate to average daily energy expenditure (DEE) and; (2) quantify the intensity at which activities of daily living (ADL) are undertaken in people with chronic obstructive pulmonary disease (COPD).

Methods A study was undertaken in 26 subjects with stable COPD (mean, $\mathrm{SD}$ ) aged 66 (7) years with an $\mathrm{FEV}_{1}$ of 50 (16) \% predicted (16 males). Measures were collected of distance walked during the six-minute walk test (6MWD) and incremental shuttle walk test (ISWD) and peak rate of oxygen uptake during a cycle ergometry test $\left(\mathrm{VO}_{2}\right.$ ak $)$. The Sensewear ArmBand $\mathrm{A}$ was worn during the waking hours for 4.4 (1.1) days to measure DEE. The intensity at which activities of daily living were undertaken was expressed as a percentage of $\mathrm{VO}_{2 p e x}$

Results DEE was associated with 6MWD $(r=0.40 ; p=0.046)$, ISWD $(r=$ $0.52 ; p=0.007)$ but not $\mathrm{VO}_{2 \text { peak }}(r=0.07 ; p=0.74)$. Stronger associations were observed between DEE and the body weight-walking product for 6MWD ( $r=$ $0.73 ; p<0.001)$ and ISWD $(r=0.75 ; p<0.001)$. The average intensity of ADL was equal to $58(12 \%)$ of $\mathrm{VO}_{2 \text { peak }}$ (range 39 to $90 \%$ ).

Conclusions 6MWD and ISWD, but not $\mathrm{VO}_{2 \text { peak }}$ were related to DEE. As $\mathrm{ADL}$ were performed at a high percentage of $\mathrm{VO}_{2 \text { peak }}$ it may be more realistic to increase DEE by increasing the frequency or duration, rather than the intensity of physical activity.

Supported by Physicians Services' Incorporated Foundation (Canada).

Conflict of Interest No.

\section{TP-165}

THE CHANGING CHARACTERISTICS OF PATIENTS REFERRED TO PULMONARY REHABILITATION: A 12-YEAR REVIEW

N CECINS ${ }^{1,2,3}$, S JENKINS ${ }^{1,2,3}$

${ }^{1}$ Physiotherapy, Sir Charles Gairdner Hospital, Perth, WA, ${ }^{2}$ School of Physiotherapy, Curtin University, Perth, WA, and ${ }^{3}$ Lung Institute of Western Australia, University of Western Australia, Perth, WA

Aim This study examined the characteristics of patients entering a pulmonary rehabilitation program (PRP) over a 12-year period.

Methods Characteristics of patients entering a hospital-based PRP from 1998 to 2003 were compared with those entering from 2004 to 2009.

Results In the most recent 6 years (2004-2009), patients entering the PRP were older and there were more females (Table). Mean BMI was similar, however, there was a higher proportion of patients in the 'overweight' range ( 28 vs. $36 \%, p=0.03$ ) and a lower proportion in the 'normal' weight range (46 vs. $37 \%, p=0.03$ ). Patients with COPD who entered between 2004 and 2009 had less severe disease.

Conclusions Modifications to the current program may be required to accommodate the older population with more comorbidities being referred to PRP

\begin{tabular}{lll}
\hline Baseline characteristics & $1998-2003(\mathrm{n}=373)$ & $2004-2009(\mathrm{n}=309)$ \\
\hline Sex (M/F \%) & $56 / 44$ & $51 / 49$ \\
Age (years) & $66(10)$ & $70(10)^{*}$ \\
Comorbidities ( $\geq 2, \%)$ & 35 & 41 \\
BMl (kg/m²) & $25.0(6.4)$ & $25.3(6.0)$ \\
GMWD (m, \%pred) & $390(125), 61(18)$ & $404(160), 64(19)$ \\
HRQoL (CRDQ, total score) & $83(25)$ & $85(18)$ \\
Adm and bed days (last 12/12) & $0.8(1.5), 7(15)$ & $0.7(1.0), 7(13)$ \\
Diagnosis of COPD (n, \%) & $284(76 \%)$ & $238(76 \%)$ \\
COPD: FEV 1 (L, \%pred) & $0.96(0.46), 38(18)$ & $1.10(0.51),{ }^{*} 45(17)^{*}$ \\
Completed PRP (n, \%) & $278(75 \%)$ & $252(81 \%){ }^{*}$ \\
\hline
\end{tabular}

BMI, body mass index; HRQoL, health related quality of life; CRDQ, Chronic Respiratory Disease Questionnaire; adm, admissions, Mean (SD), ${ }^{*} p<0.01$

Conflict of Interest No. 
PREVALENCE OF REDUCED 6-MINUTE WALK DISTANCE (6MWD) IN COPD; DIFFERENCES WITH DISEASE SEVERITY AND GENDER

$\mathrm{K} \mathrm{HILL}^{1,2,4,5}$, D BROOKS ${ }^{4,5}$, L WICKERSON ${ }^{4}$, L WOON $^{4}, \mathrm{~N} \mathrm{CECINS}^{1,2,6}$, P EASTWOOD ${ }^{1,3,7}$, R GOLDSTEIN ${ }^{4,5}$, P THOMPSON ${ }^{1,2}$, S JENKINS ${ }^{1,2,6}$ ${ }^{1}$ Physiotherapy, Curtin University, Bentley, WA, 6845, '2Lung Institute of Western Australia and, ${ }^{3}$ Anatomy and Human Biology, University of Western Australia, Perth, WA, ${ }^{4}$ Respirology, West Park Healthcare Centre, Ontario, Canada, ${ }^{5}$ Physical Therapy and Medicine, University of Toronto, Ontario, Canada, ${ }^{6}$ Physiotherapy and, ${ }^{7}$ Pulmonary Physiology, Sir Charles Gairdner Hospital, WA

Aim To determine; (1) the lower limit of normal (LLN) for 6MWD, defined as a distance below the 5th percentile in a healthy population and, (2) the prevalence of people with COPD who have reduced 6MWD, defined as a distance below the LLN.

Methods Equations were developed to estimate normal 6MWD using data from 186 healthy individuals aged 63 (10) year (mean, SD). The prevalence of reduced 6MWD was examined in 217 COPD patients referred for rehabilitation aged 67 (9) year.

Results Equations were; Males: $6 \mathrm{MWD}=1138.4+(-5.6 \times$ age $)+(-3.8 \times$ body mass index $(\mathrm{BMI}))\left(\mathrm{r}^{2}=0.43\right)$ and Females: $6 \mathrm{MWD}=1142.9+(-4.9 \times$ age $)+(-8.3 \times \mathrm{BMI})\left(\mathrm{r}^{2}=0.49\right)$. Reduced 6MWD was evident in $179(82 \%)$ COPD patients, being similar in males and females ( $84 \%$ vs. $80 \% ; p=0.55)$. With patients grouped according to disease severity, $63(29 \%), 92(42 \%)$ and $62(29 \%)$ were classified as GOLD stage $1+2,3$ and 4 , respectively. The prevalence of reduced 6MWD increased with GOLD stage, being $71 \%, 83 \%$ and $94 \%$ at stages $1+2,3$ and 4 , respectively $(p=0.005)$.

Conclusions Reduced 6MWD is evident in the majority of patients, even in those with mild to moderate disease. The prevalence of reduced 6MWD in people with COPD increased with advancing disease but did not differ between genders.

Supported by Ontario Respiratory Care Society.

Conflict of Interest No.

\section{PREVALENCE OF CARDIOVASCULAR (CV) MEDICATION USE IN COPD PATIENTS PARTICIPATING IN PULMONARY REHABILATATION (PR)}

B NOTEBOOM ${ }^{1,2,3}$, S JENKINS ${ }^{1,3,4}$, C NG $^{1,3,4}$, N CECINS $^{1,3,4}$, A MAIORANA ${ }^{1,5}, \mathrm{~K}^{1}$ HILL $^{1,3}$

${ }^{1}$ Physiotherapy, Curtin University, Bentley, WA, ${ }^{2}$ Physiotherapy and ${ }^{5}$ Cardiac Transplant and Advanced Heart Failure Service, Royal Perth Hospital, Perth, WA, ${ }^{3}$ Lung Institute of Western Australia, University of Western Australia, Perth, WA, ${ }^{4}$ Physiotherapy, Sir Charles Gairdner Hospital, Perth, WA

Although people with COPD are at greater risk of death from CV causes compared with the general population, the prevalence of $\mathrm{CV}$ medication use is not well documented.

Aim To determine the prevalence of CV medication use in patients with COPD currently participating in outpatient PR.

Methods Patients participating in PR at four hospitals were asked to provide a list of their current medications. Age, body-mass index (BMI), FEV $\%$ pred and 6-minute walk distance (6MWD) data were acquired from medical records. Results Data were available on 75 patients (mean, SD) aged 70 (9) year with an FEV 1 of $41(16) \%$ pred, of whom $45 \%$ were male. There was a large range in BMI (14 to $38 \mathrm{~kg} \cdot \mathrm{m}^{-2}$ ) and 6MWD (40 to $632 \mathrm{~m}$ ). Twenty five (33\%) patients were not on any CV medication, $14(19 \%)$ were on one CV medication and $36(48 \%)$ were on 2 or more. The most frequently used CV medications are summarized below.

\begin{tabular}{lccccc}
\hline Medication & Statin & $\begin{array}{c}\text { Anti-platelet/ } \\
\text { coagulant }\end{array}$ & $\begin{array}{c}\text { Ca channel } \\
\text { blocker }\end{array}$ & Diuretic & Nitrate \\
\hline $\mathrm{n}(\%)$ & $34(45 \%)$ & $24(32 \%)$ & $21(28 \%)$ & $19(25 \%)$ & $14(19 \%)$ \\
\hline
\end{tabular}

Compared with GOLD stages $1+2$ and 3 , there was a smaller proportion of patients in GOLD stage 4 using at least one CV medication $(74 \%, 80 \%$ vs. $38 \% ; p=0.004)$.

Conclusions The majority of patients with COPD participating in PR have been prescribed $\mathrm{CV}$ medications. The smaller proportion of patients on a CV medication in GOLD stage 4 may reflect a healthy survivor effect.

Conflict of Interest No.

\section{TP-169}

TP-167

\section{EXERCISE INTENSITY OF SUN-STYLE TAI CHI IN PEOPLE WITH COPD}

RWM LEUNG ${ }^{1,2}$, JA ALISON $^{1}$ ZJ MCKEOUGH ${ }^{1}$, MJ PETERS $^{3}$

${ }^{1}$ Discipline of Physiotherapy, The University of Sydney, NSW, ${ }^{2}$ Department of Physiotherapy, Concord Hospital, NSW, and ${ }^{3}$ Respiratory Medicine, Concord Hospital, NSW

Introduction There is increasing interest in Tai Chi as a training mode for people with COPD but the exercise intensity of Tai Chi is unknown.

Aim To investigate the exercise intensity of Sun-style Tai Chi in people with COPD.

Methods Participants with COPD who had completed 12 weeks of Sun-style Tai Chi training were recruited. Oxygen consumption $\left(\mathrm{VO}_{2}\right)$ during the incremental shuttle walk test (ISWT) and during 15 minutes of Tai Chi was measured using a portable gas analysis system (Cosmed k4b2). The exercise intensity during Tai Chi was determined by comparing the average $\mathrm{VO}_{2}$ during the last 14 minutes of Tai Chi to the peak $\mathrm{VO}_{2}$ of the ISWT. Heart rate during Tai Chi was compared to peak heart rate from ISWT.

Results Six participants with COPD (mean (SD) age 76 (10) years and FEV 1 65 (17) \% predicted) completed the study. The mean $\mathrm{VO}_{2}$ during Tai Chi training was $0.66(0.14) \mathrm{L} / \mathrm{min}$ and the mean peak $\mathrm{VO}_{2}$ of ISWT was $1.08(0.14)$ $\mathrm{L} / \mathrm{min}$. The VO $\mathrm{VO}_{2}$ during Tai Chi was $62(19) \%$ peak VO 2 . The exercise intensity of Tai Chi was greater than $60 \%$ peak $\mathrm{VO}_{2}$ in 3 participants and between $45 \%$ and $52 \%$ peak $\mathrm{VO}_{2}$ in 3 participants. The heart rate (HR) during Tai Chi was $89(15) \%$ of peak HR in ISWT.

Conclusions The exercise intensity of Sun-style Tai Chi was moderate, and was within the recommended exercise training intensity of $60 \%$ peak $\mathrm{VO}_{2}$, which is known to elicit physiological training responses in people with COPD. However, the variation in $\mathrm{VO}_{2}$ between participants performing Tai Chi needs to be evaluated in a larger cohort.

Supported by Physiotherapy Research Foundation and Physiotherapy Registration Board.

Nomination No.

Conflict of Interest No.

\section{COMMUNITY-BASED PULMONARY REHABILITATION: A PILOT PROGRAM IN A RECREATIONAL FACILITY}

\section{N CECINS ${ }^{1,2,3}$, J APPLEBEE ${ }^{2}$, A HAMERSLEY ${ }^{2}$, L GANDERTON ${ }^{2,3}$,} $J^{J}$ COCKRAM $^{2}$, B MIDDLETON ${ }^{2}$

${ }^{1}$ Physiotherapy, Sir Charles Gairdner Hospital, Perth, WA, ${ }^{2}$ Community Physiotherapy Services, Perth, WA, and ${ }^{3}$ Lung Institute of Western Australia, University of Western Australia, Perth, WA

Pulmonary rehabilitation programs (PRP) provide a non-pharmacological, cost effective, beneficial intervention for patients with chronic lung disease. Access to initial programs is variable in Western Australia and mostly provided by secondary and tertiary hospitals. Reasons for poor uptake and attrition from these programs include: too far to travel, inadequate parking and poor access to public transport.

Aim To determine the feasibility and safety of conducting a PRP in a recreational facility for individuals with chronic lung disease at a low-risk of adverse events.

Methods Individuals referred to a tertiary hospital outpatient PRP were screened for suitability to attend a community-based PRP. Inclusion criteria comprised: diagnosis of chronic lung disease and limited by breathlessness; resting oxygen saturation $\left(\mathrm{SpO}_{2}\right) \geq 92 \%$; low cardiovascular risk; motivated and willing to attend a community program. Exclusion criteria comprised: $\mathrm{SpO}_{2}$ $<85 \%$ on 6 -minute walk test; severe musculoskeletal or neurological condition impacting on ability to exercise. Suitable individuals were invited to attend the PRP, assessed and entered an 8-week program (2 supervised sessions + home exercise program). The PRP comprised a 20-minute walking program, and upper and lower limb exercises requiring minimal equipment. Informal education sessions were incorporated into the program. A physiotherapist conducted the PRP.

Results To date, 10 individuals (mean age $70,60-85$ years), were deemed suitable for the PRP. Eight had a diagnosis of COPD (mean, SD FEV ${ }_{1}$ 0.82L $(0.19), 32(5) \% p r e d)$. Two individuals were not suitable and referred to hydrotherapy. Mean baseline 6-minute walk distance was 416 (43)m.

Conclusions It is anticipated that this pilot program will provide evidence for the feasibility and safety of PRPs conducted in recreational facilities.

Conflict of Interest No.

Journal Compilation @ 2011 Asian Pacific Society of Respirology 
CHARACTERISTICS OF IMPAIRMENT AND DISABILITY IN INDIVIDUALS WITH IDIOPATHIC PULMONARY FIBROSIS (IPF) STRATIFIED BY MEDICAL RESEARCH COUNCIL (MRC) DYSPNOEA GRADE

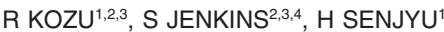

${ }^{1}$ Rehabilitation Medicine, Nagasaki University, Nagasaki, Japan,

${ }^{2}$ Physiotherapy, Sir Charles Gairdner Hospital, WA, ${ }^{3}$ School of

Physiotherapy, Curtin University, WA, and ${ }^{4}$ Lung Institute of Western

Australia, University of Western Australia, WA

Aim To compare measures of impairment and disability in individuals with IPF stratified by the MRC Dyspnoea Grade.

Methods 65 subjects (46 males), aged 68 (7) years, with stable IPF were evaluated in this study. Demographic data and measures of pulmonary function (spirometry, diffusing capacity for carbon monoxide, $\left(\mathrm{DL}_{\mathrm{co}}\right)$ ), dyspnoea (Baseline Dyspnoea Index, BDI), peripheral muscle force (isometric quadriceps force (QF) and handgrip force (HF)), functional exercise capacity (6-minute walk distance, 6MWD), limitation in daily activities (Activities of Daily Living (ADL) score), and health status (SF-36) were assessed. Relationships between $6 \mathrm{MWD}$ and MRC Grade, pulmonary function, QF, BDI and ADL score were examined.

Results The number of subjects in MRC Grades 2, 3, 4 and 5 was $16(25 \%)$, $17(26 \%), 17(26 \%)$ and $15(23 \%)$, respectively. Pulmonary function, BDI, QF, HF, 6MWD, ADL score, and SF-36 decreased significantly with increasing MRC Grade (all $p<0.05)$. Moderate to strong correlations were found between 6MWD and MRC Grade $(r=-0.89), \mathrm{DL}_{\mathrm{co}}(\mathrm{r}=0.67)$, QF $(r=0.66)$, BDI $(r=$ 0.87 ) and $A D L$ score $(r=0.85)$ (all $p<0.005$ ).

Conclusions These findings suggest that the MRC Dyspnoea Scale can be used to discriminate and classify subjects with IPF according to the severity of impairment and disability.

Conflict of Interest No.

\section{TRACHEOSTOMY WEANING IN A RESPIRATORY HIGH DEPENDENCY UNIT}

K NEHYBA 1 , I LING ${ }^{2}$, B SINGH ${ }^{2}$

Departments of ${ }^{1}$ Physiotherapy and ${ }^{2}$ Pulmonary Physiology, Sir Charles Gairdner Hospital, WA, 6009

Background Patients with tracheostomies have high clinical acuity and complex care needs. Tracheostomy weaning is best individualized and undertaken by staff with specialist knowledge and skills. The literature identifies differences in outcomes for tracheostomy weaning.

Aim To examine the characteristics and outcomes of tracheostomy patients managed by a Respiratory High Dependency Unit (RHDU) at an adult tertiary hospital and to compare this with published reports of tracheostomy weaning. Methods Data were collected prospectively for all patients with a tracheostomy admitted to the RHDU between August 2009 and August 2010.

Results Tracheostomy weaning was initiated and completed by the RHDU in 22 patients. Time to decannulation, reported complications and discharge location compared favourably with published data. There was an increase in admissions for tracheostomy weaning in 2009 associated with H1N1 influenza activity. Conclusion Tracheostomy weaning in a specialized RHDU is safe and effective.

Conflict of Interest None.

\begin{tabular}{ll}
\hline & \multicolumn{1}{c}{$\mathrm{n}=22$} \\
\hline Age (years) & $54.5(23-79)$ \\
Males (n, \%) & $10,45 \%$ \\
Time: RHDU admission to cuff deflation (days) & $1(0-5)$ \\
Time: RHDU admission to decannulation (days) & $5.5(1-19)$ \\
Time: tracheostomy insertion to decannulation (days) & $15(6-27)$ \\
Discharged home (n, \%) & $10,45 \%$ \\
Readmission to Intensive Care (n, \%) & $1,4.5 \%$ \\
In-hospital mortality & Nil \\
\hline
\end{tabular}

Median (range)

RESPIRATORY INFECTIOUS DISEASES SIG: POSTER SESSION

TP-173

\section{OXIDATIVE STRESS INDUCES MITOCHONDRIAL DYSFUNCTION IN AIRWAY EPITHELIAL CELLS AND IMPAIRS RESPONSE TO RHINOVIRUS}

K PARSONS $^{1}$, M TOOZE $^{1}$, A HSU $^{1}$, PAB WARK $^{1,2}$

${ }^{1}$ Centre for Asthma and Respiratory Disease, Hunter Medical Research Institute, University of Newcastle, NSW, and ${ }^{2}$ Department of Respiratory and Sleep Medicine John Hunter Hospital, Newcastle, NSW

Infection with rhinovirus (RV) is known to trigger acute exacerbations in subjects with asthma and these subjects also have increased susceptibility to the effects of RV. The mechanisms remain poorly understood, but appear to involve a host innate immune defect in the airway epithelium.

Aim We sought to determine in bronchial epithelial cells (BECs) if oxidative stress in the form of exposure to cigarette smoke extract (CSE), hydrogen peroxide $\left(\mathrm{H}_{2} \mathrm{O}_{2}\right)$ and eosinophil peroxidise (EPO) results in impaired mitochondrial function and if this directly impairs signalling of $R V$ infection through MDA5 and alters the release of type I and type III interferons (IFNs).

Methods pBECs were grown to confluence. Cells were then exposed to CSE (1\%, no filter) or $\mathrm{H}_{2} \mathrm{O}_{2}(0.2 \mathrm{mM})$ or EPO. Cells were then infected with RV1-B $(\mathrm{MOI}=20)$. Virus replication was measured by cell titration assay. Following infection, IL-6, CXCL-8, CXCL-10 was measured using cytometric bead array and flow cytometry. Supernatants and whole cell lysates were collected for IFN- $\beta$, BAX and MDA5 detection by western blot. IFN- $\lambda$ and cytochrome-c was measured using conventional ELISA. Cell viability was assessed by Annexin V-PE staining and flow cytometry.

Results RV infection alone induced CXCL-8, IL-6, CXCL-10 and IFN- $\lambda$. pBECs treated with each of the oxidative stressors had increased cytochrome$\mathrm{C}$ release and increased apoptosis. This mitochondrial dysfunction led to degradation of MDA5 expression and resulted in specific suppression of CXCL-10 and IFN- $\lambda$.

Conclusions Exposure of BECs to an oxidative stress results in mitochondrial dysfunction in airway epithelial cells. This leads to defective antiviral signalling in the airway epithelium after infection with RV.

Supported by the NHMRC Australia.

Nomination Nil.

Conflict of Interest None. 
INTRAPLEURAL TISSUE PLASMINOGEN ACTIVATOR (T-PA) AND DEOXYRIBONUCLEASE (DNASE) ENHANCE PLEURAL DRAINAGE IN EMPYEMA

\author{
F PICCOLO 1 , RL SHRESTHA ${ }^{1}$, L CRUICKSHANK ${ }^{1}$, N POPOWICZ $^{2}$, \\ YCG LEE LE, $^{1,3}$ \\ ${ }^{1}$ Departments of Respiratory Medicine and ${ }^{2}$ Pharmacy, Sir Charles Gairdner \\ Hospital, WA, Australia, and ${ }^{3}$ Centre for Asthma, Allergy and Respiratory \\ Research and School of Medicine and Pharmacology, University of Western \\ Australia
}

Introduction Pleural infection is associated with high morbidity. Prompt drainage is key, but pus is often loculated and thick making drainage difficult. Based on promising animal studies, we hypothesize that intrapleural therapy with t-PA and DNase, which lyse adhesions and reduce fluid viscosity respectively, can significantly improve pus evacuation in pleural infection.

Methods Consecutive patients with pleural infection were treated with standard antibiotics and intercostal chest tube (ICT) drainage. Additionally, t-PA $10 \mathrm{mg}$ and DNase $5 \mathrm{mg}$ (each in $50 \mathrm{~mL}$ of $0.9 \% \mathrm{NaCl}$ ) were instilled intrapleurally via an ICT twice daily for up to six doses. The ICT was clamped for 45 minutes after each instillation. Patients were followed clinically and with serial CXR. Opacity from pleural effusion was quantified on chest radiographs.

Results Eleven patients (8 male; mean age 51) were treated. Nine effusions were associated with community acquired pneumonia, of these, eight were visibly purulent, five were culture positive and the mean fluid $\mathrm{pH}$ was 7.0 (range $6.45-7.50)$. Ten patients $(91 \%)$ were successfully managed conservatively and one patient required surgery. Median hospital stay from first intrapleural treatment dose to discharge was 7 days (range 4-20). The median amount of fluid drained in the 24 hours preceding t-PA/DNase treatment was $180 \mathrm{~mL}$ (range $0-1365$ ), and improved significantly to $1475 \mathrm{~mL}$ (range 200-4150) following two doses of treatment. This was paralleled by a significant reduction in radiographic opacity by a mean value of $26 \%$ of the hemithorax (range $10-48 \%$ ). Four patients showed an initial rise in CRP following t-PA/DNase, but all patients had resolution of sepsis and significant reduction in CRP. There were no major complications. Pleuritic chest pain requiring opioid analgesia developed in three patients.

Conclusion Intrapleural combination of tPA/DNase is practical, safe and enhances pleural drainage in empyema.

Support and Conflict of Interest Nil.

\section{TP-175}

\section{CLINICAL SIGNS AND WHO-DEFINED XRAY-CONFIRMED PNEUMONIA IN CENTRAL AUSTRALIAN ABORIGINAL CHILDREN}

KF O'GRADY', PJ TORZILLO ${ }^{2,3}$, A RUBEN $N^{4,5}$, P VALERY $^{6}$, AB CHANG $^{1,7}$ ${ }^{1}$ Queensland Children's Medical Research Institute (QCMRI), QId, ${ }^{2}$ Department of Respiratory Medicine, University of Sydney, NSW, ${ }^{3}$ Royal Prince Alfred Hospital, NSW, ${ }^{4}$ Northern Territory Clinical School, NT, ${ }^{5}$ Northern Territory Department of Health \& Families, NT, ${ }^{6}$ Queensland Institute of Medical Research, Qld, and ${ }^{7}$ Menzies School of Health Research, NT

Aim To evaluate the World Health Organization's (WHO) definition of xrayconfirmed lobar pneumonia in the clinical context in Central Australian Aboriginal children hospitalized with acute lower respiratory illness (ALRI) and treated with IV antibiotics.

Methods Clinical data were collected using a standardized form for Aboriginal children aged 29 days $-<60$ months hospitalized with ALRI and enrolled in a RCT of Vitamin A/Zinc supplementation were matched with data collected during a population-based study of WHO-defined primary endpoint pneumonia (WHO-P). Sensitivities, specificities, positive and negative predictive values (PPV, NPV) for these signs were compared between WHO-P cases and lobar pneumonia assigned by a respiratory paediatrician.

Results In 147 episodes of hospitalized ALRI, WHO-P was diagnosed in 40 $(27.2 \%)$; the respiratory paediatrician classified $70(47.6 \%)$ as lobar pneumonia. The sensitivities of clinical signs ranged from a high of $45 \%$ for tachypnoea to $5 \%$ for fever + tachypnoea + chest-indrawing; the PPV range was $40 \%$ to $20 \%$, respectively. Higher PPVs were observed against the paediatric respiratory physicians diagnosis compared to WHO-P.

Conclusions Clinical signs on admission are not useful in predicting WHO-P in this population, presenting challenges for future pneumonia research in this population. WHO-P may underestimate alveolar consolidation in a clinical context and its use in clinical practice or in research designed to inform clinical management in this population should be avoided.

Supported by KOG and AC are supported by NHMRC Fellowships (grant numbers: 490338 and 545216).

Conflict of Interest None.

\section{INDUCED SPUTUM STILL HAS A ROLE IN INCREASING DIAGNOSTIC YIELD IN THE INVESTIGATION OF PULMONARY TUBERCULOSIS IN SELECTED HIGH RISK PATIENTS WITHIN LOW PREVALENCE COMMUNITIES}

B MUPUNGA ${ }^{1}$, GW EATHER ${ }^{1,2}$, A KONSTANTINOS ${ }^{2}$

${ }^{1}$ Department of Respiratory and Sleep Medicine, Princess Alexandra

Hospital, Ipswich Rd, Woolloongabba, Qld, Australia, and²Queensland

Tuberculosis Control Centre, Cornwall St, Annerley, QId, Australia

Aim To determine whether induced sputum production increases diagnostic yield in patients being investigated for pulmonary tuberculosis (PTB) who have had either difficulties producing adequate sputum samples, or failure to isolate M. tuberculosis on standard sputum smears and cultures.

Methods All cases of suspected PTB who were smear and culture negative on standard, spontaneously produced sputum samples during the period from April 2007 to April 2009, were included in a retrospective chart review.

Results 74 patients met the study criteria. The age range was between 18 and 83 years with equal gender distribution. Forty-eight $(65 \%)$ of cases were migrants from high tuberculosis prevalent regions of the world such as the Indian subcontinent and Africa. Fifty-four (73\%) of cases had 2 or more standard sputum smear examinations and culture while $22(27 \%)$ had one or less sputum submitted. Fifty-nine (79\%) of all cases had abnormal chest radiographs. Induced sputum smear and culture isolated $M$. tuberculosis in an extra $31(41 \%)$ of the initially culture-negative patients. Bronchoscopy was performed in a further 4 patients adding an extra 3 PTB cases. $4(5 \%)$ were diagnosed as clinical PTB, $14(20 \%)$ have annual follow-up; 19(26\%) discharged; and $2(3 \%)$ returned to countries of origin.

Conclusions Induced sputum increased the diagnostic yield in patients with suspected PTB by a magnitude of $31(41 \%)$ of patients over a 2 -year period. Induced sputum examination potentially saved $31(41 \%)$ fibre-optic bronchoscope examinations with the avoidance of the potentially added risks to patients and staff.

Conflict of Interest None.

Acknowledgements Staff of Queensland Tuberculosis Control Centre, Qld.

\section{MARIJUANA 'BONG' SMOKING AND TUBERCULOSIS}

K THU, M HAYES

Department of General Medicine, Calvary Mater Newcastle, NSW

The incidence of TB in the non-Indigenous Australian population is uncommon at 0.9 cases per 100000 population 1 . In this paper, we report three cases of pulmonary tuberculosis in young Australian born, non-indigenous adults in the Hunter New England area where marijuana possibly was a significant risk factor in transmission and severity of disease. All three cases had severe cavitating disease at time of presentation. Contact tracing from the first case, a regular heavy marijuana user, identified 26 Mantoux positive contacts, one of whom developed active pulmonary tuberculosis. All contacts, mainly young adult males, denied sharing marijuana with the index case. Contact tracing from the second case identified 5 Mantoux positive contacts, 4 of whom use marijuana regularly and shared bongs (water pipes) with the index case. There were 3 positive Mantoux contacts of the third case, one of whom shared bongs with the index case. Health professionals need to remain aware of the possibility of tuberculosis in groups with historically low incidence rates. Marijuana bong smoking is possibly associated with transmission and severity of tuberculosis ${ }^{2}$

Conflict of Interest No.

References

1 Barry C, Konstantinos A et al. uberculosis notifications in Australia 2007 Commun Dis Intell 2009; 33 (3) 304-15.

2 Munckhof WJ, Konstantinos A, Wamsley M, et al. A cluster of tuberculosis associated with use of a marijuana water pipe. Int J Tuberc Lung Dis 2003; 7: 860-5.

Journal Compilation @ 2011 Asian Pacific Society of Respirology 
TP-178

HEAT SHOCK PROTEIN 70 IS RELEASED FROM MESOTHELIAL CELLS AND ELEVATED IN INFECTIOUS PLEURAL EFFUSIONS

JF VARANO DELLA VERGILIANA ${ }^{1,2}$, S LANSLEY ${ }^{1,2}$, J CREANEY ${ }^{3}$, $S$ TEMPLE $^{4}$, G WATERER ${ }^{4}$, YCG LEE ${ }^{5}$

${ }^{1}$ Centre for Asthma, Allergy and Respiratory Research, School of Medicine \& Pharmacology, University of Western Australia, Perth, Western Australia, ${ }^{2}$ Lung Institute of Western Australia, University of Western Australia, Perth, Western Australia, ${ }^{3}$ National Research Centre for Asbestos Related Diseases, ${ }^{4}$ Western Australia Institute for Medical Research, University of Western Australia, Perth, Western Australia, and ${ }^{5}$ Respiratory Dept, Sir Charles Gairdner Hospital, Perth, Western Australia

Aim To determine if heat shock protein 70 (HSP70) is present in pleural effusions of various etiologies and whether bacterial stimulation of pleural mesothelial cells induces HSP70 release.

Methods Pleural effusions were obtained from patients with a range of common pleural etiologies and HSP70 concentrations determined by ELISA The benign MeT-5A pleural mesothelial cell line was treated with live or heat killed Streptococcus pneumoniae and HSP70 release determined.

Results HSP70 concentrations were 7.25-fold higher in pleural fluids compared to the corresponding serum samples. When compared to all other etiologies, pleural fluid HSP70 concentrations were higher in patients with pleural infection, which were further elevated in patients with empyema. At concentration which did not cause cell death, live and heat killed $S$. pneumoniae induced significant HSP70 release from MeT-5A cells.

Conclusions HSP70 is released from pleural mesothelial cells in response to bacterial stimulation and may contribute to the elevated HSP70 concentrations observed in infectious pleural effusions. This may present a potential target for anti-inflammatory therapies in serosal inflammation.

Supported by Australian NHMRC.

Conflict of Interest None.
STREPTOCOCCUS PYOGENES: AN UNUSUAL ORGANISM CAUSING SEVERE COMMUNITY ACQUIRED PNEUMONIA IN TWO YOUNG WOMEN WHO SURVIVED FOLLOWING INTERHOSPITAL TRANSFER ON EXTRACORPOREAL MEMBRANE OXYGENATION (ECMO)

SE MILES 1,2, AB TIERNEY ${ }^{1,3}$ PA WILSON ${ }^{1,3}$, PJ TORZILLO ${ }^{4}$, RG HERKES ${ }^{5}$, AP FORREST ${ }^{6}$

${ }^{1}$ Department of General Medicine, Calvary Mater Newcastle, NSW, Australia, ${ }^{2}$ Department of Respiratory and Sleep Medicine, John Hunter Hospital, Newcastle, NSW, ${ }^{3}$ Department of Infectious Diseases and Immunology, John Hunter Hospital, ${ }^{4}$ Department of Respiratory Medicine, ${ }^{5}$ Intensive Care Service, and ${ }^{6}$ Department of Anaesthetics, Royal Prince Alfred Hospital, Sydney NSW

Introduction In 2007, these previously well women survived and made a good recovery from severe pneumonia and acute lung injury after retrieval on ECMO. Streptococcus pyogenes is an unusual cause of pneumonia in adults. Case 1 A 22-year-old veterinarian with a history of mild asthma presented with 4 days of fever and respiratory symptoms. The diagnosis was confirmed by a fourfold rise in the anti-streptococcal antibody. This was complicated by respiratory failure, septic shock, acute renal failure, severe pulmonary hypertension and bilateral parapneumonic effusions. Despite maximal interventions she deteriorated. Femoral venous-venous ECMO was initiated on day 5 at the Calvary Mater Hospital in Newcastle by a retrieval team from Royal Prince Alfred Hospital (RPA), Sydney. She was transferred $158 \mathrm{kms}$ on ECMO in a large multipurpose ambulance. She developed lung abscesses and recurrent pneumothoraces and she required a pleurodesis. She required 40 days of ventilation and 15 days of ECMO. Three months later she was asymptomatic, with mildly restrictive spirometry and minor CXR change.

Case 2 A 47-year-old office worker with $S$ pyogenes bacteraemia made a similar presentation to our institution. She was ventilated for 24 days, ECMO was initiated by the retrieval team and continued for 7 days. Three months later she was asymptomatic with a normal CXR and pulmonary function tests. Case 1 was the first intercity transfer on ECMO in NSW. There have been 46 interhospital transfers on ECMO in NSW since 2007. Since May, 2009 a formal ECMO retrieval service has operated in NSW, as a collaboration between RPA and St. Vincent's Hospital, Sydney.

Conflict of Interest No.

\section{TP-179}

LOW PREVALENCE OF POSITIVE URINARY PNEUMOCOCCAL ANTIGEN TESTS IN COMMUNITY ACQUIRED PNEUMONIA

$\mathrm{L}^{\mathrm{TROY}}{ }^{1}, \mathrm{~K}$ WONG $^{1,2}, \mathrm{R}_{\mathrm{CHAN}}{ }^{3}, \mathrm{D}^{\mathrm{B}} \mathrm{BARNES}^{1}$

${ }^{1}$ Respiratory Medicine, Royal Prince Alfred Hospital, NSW, Australia,

${ }^{2}$ Woolcock Institute of Medical Research, NSW, Australia, ${ }^{3}$ Microbiology \&

Infectious Diseases, Royal Prince Alfred Hospital, NSW, Australia

Introduction The urinary pneumococcal antigen (UPA) test has been shown to have superior sensitivity to other investigations in determining the aetiology of community-acquired pneumonia (CAP), but there is very limited data on its performance in local populations. The aims of this study are to establish the prevalence of positive UPA testing in patients admitted to hospital with CAP, and determine its utility. Secondary aims are to identify associations with positive testing, as well as to determine if a positive test influences clinical outcomes.

Methods The study is a prospective, single-centre study that is still recruiting. Adult patients are included upon admission to hospital if they have the diagnosis of CAP, as defined by new infiltrates on chest radiograph along with consistent clinical features. Clinical data including CURB-65 score of severity, current and prior antibiotics, co-morbidities, mortality and length of hospital stay are recorded.

Results Preliminary results show a positive test prevalence of 9/124 (7.3\%, 95\% Cl 3.6-13.7\%) amongst patients admitted with CAP. Overall prevalence of pneumococcal pneumonia is $12 / 124(9.7 \%, \mathrm{Cl} 5.3-16.7 \%)$. Patients with a positive UPA result have a higher mean CURB-65 score of 2.7 compared with 1.5 in those with a negative result $(p=0.02) .44 .4 \%$ of patients with a positive result were admitted to the intensive care unit, compared with $13.0 \%$ those with a negative result $(p=0.04)$.

Conclusions The overall prevalence of positive UPA testing in patients admitted to hospital with CAP is low. Preliminary data suggests that patients with positive results are more likely to have greater severity pneumonia and to require intensive care support. Comparative data on length of stay, mortality, previous antibiotic use and specific co-morbidities has not revealed any statistically significant differences between positive and negative groups.

Conflict of Interests No.
TP-181

\section{RHODOCOCCUS EQUI PULMONARY INFECTION IN AN IMMUNO-COMPETENT HOST: A CASE REPORT AND REVIEW OF LITERATURE}

\section{S HERATH ${ }^{1}$, C LEWIS $^{2}$, M NISBET $^{1,2}$}

${ }^{1}$ Respiratory Department, Auckland City Hospital, Auckland, New Zealand, and ${ }^{2}$ Infectious Diseases Department, Auckland City Hospital, Auckland, New Zealand

Rhodococcus equi (R. equi), previously known as Corynebacterium equi is a Gram positive bacillus that is found in soil and causes infection in grazing livestock. It is infrequently isolated from clinical specimens. It is usually associated with human disease in immunocompromised patients and is an uncommon cause of infection in immunocompetent patients. Infection is usually acquired by the airborne route with pneumonia being the most common manifestation but it can also be acquired orally or by direct inoculation. We present a case of pneumonia caused by $R$. equi infection in a 55 year old male builder who presented with cough, dyspnoea and night sweats. $R$. equi was cultured from a transbronchial aspirate from a subcarinal lymph node. Despite extensive investigation, no contributing host immune defect was identified. The patient recovered after three months of antibiotic treatment, initially with intravenous vancomycin and meropenem followed by oral clarithromycin and rifampicin. Although infections due to $R$. equi have been increasingly reported in immunocompromised patients, since 1989 there have only been 24 cases described in patients where no associated host immune defect was reported. In this cohort, the median age at presentation was 53 years (range 16-83) and $18(72 \%)$ patients were male. Ten $(42 \%)$ of these cases had pulmonary infection. Two $(8 \%)$ patients died and the remainder were successfully treated with prolonged antibiotics. $R$. equi is an uncommon cause of infection in humans and rarely occurs in patients where a host immune defect cannot be identified.

Conflict of Interest No. 


\section{PULMONARY INVOLVEMENT IN PATIENTS PRESENTING WITH EXTRA PULMONARY TUBERCULOSIS: THINKING BEYOND A NORMAL CXR}

\author{
$S$ HERATH, C LEWIS \\ Department of Respiratory Medicine, Auckland City Hospital, Auckland, \\ New Zealand
}

Introduction Recognition of pulmonary involvement in Extra Pulmonary Tuberculosis (EP-TB) may be an important public health issue, as it has been estimated that patients with smear negative Pulmonary TB (PTB) are responsible for $17 \%$ of new infections. Usually, all patients with EP-TB have a chest $x$-ray but sputum cultures are requested only if there is an abnormality. Methods In this retrospective clinical audit, we aimed to evaluate the percentage of EP-TB patients with PTB despite a normal Chest X Ray (CXR), and to explore any clinical characteristics of this group. Clinical notes, microwith EP-TB between 2007 and 2010

Results Of 103 patients with EP-TB, $47 \%$ were male and the mean age was 41 (range 16 to 98). Most patients were of Asian ethnicity $(n=70,68 \%)$. The commonest presentation of EP-TB was lymphadenopathy $(n=51,50 \%)$, followed by pleural $(n=2423 \%)$ and bone $(n=6,5.8 \%)$ disease. EP-TB was diagnosed by biopsy/excision of the EP site in the majority $(n=77,74.8 \%)$, in $n=67,(88 \%)$ overall, with $n=46(67 \%)$ being positive. There was higher inflammatory markers in the sputum culture positive group (ESR 64.2 vs. 37.7, $p=0.0088$ and CRP 50.8 vs. 28.2, $p=0.0203$ ). The majority had CXR abnormalities $(n=76,74 \%)$. In the group with normal CXR $(n=27), 15(55 \%)$ had sputum cultures performed. Of these, 5 were culture positive and 2 of these also $1+$ smear positive (1 on immunosuppression, 1 with cough).

Conclusion A small number of patients with EP-TB and normal CXR had pulmonary TB, of whom 2 were smear positive. Thus, induced sputum testing should be considered in patients with EP-TB even if CXR is normal. This may aid diagnosis and determine infectivity.

Conflict of Interest No. biology and CXR reports were reviewed from consecutive patients presenting and by sputum testing alone in $26(25.2 \%)$. Sputum cultures were performed

\section{NONTUBERCULOUS MYCOBACTERIA (NTM) IN THE BRISBANE POTABLE WATER DISTRIBUTION SYSTEM}

\author{
R THOMSON $N^{1,2,3}$, C TOLSON ${ }^{4}$, R CARTER $^{4}$, C COULTER $^{4}$, F HUYGENS $^{5}$,
} M HARGREAVES ${ }^{5}$

${ }^{1}$ Thoracic Medicine, The Prince Charles Hospital, ${ }^{2} Q L D$ TB Control Centre, ${ }^{3}$ Greenslopes Private Hospital, ${ }^{4}$ QLD Mycobacterial Reference Laboratory, and ${ }^{5}$ Queensland University of Technology, Qld, Australia

NTM are normal inhabitants of environmental reservoirs including water. Disease due to NTM has been increasing in QLD. ${ }^{1}$

Aim To document the presence of NTM in potable water in Brisbane, to compare the species isolated during summer and winter and to relate this to the geographic distribution of patients with NTM.

Methods Water samples (1L) were collected from 196 routine collection sites in winter 2007 and 190 sites in summer 2008. Samples were processed in triplicate as previously described. ${ }^{2} 7 \mathrm{H} 11$ subcultures were taken from positive specimens, DNA extracted, followed by $16 \mathrm{~s}$ rRNA sequencing. Patient addresses were obtained from the QLD TB control centre database.

Results Of 1960 winter specimens, 847 (43.2\%) were positive, $397(20.3 \%)$ positive with contaminants, $188(9.6 \%)$ overgrown and $528(26.9 \%)$ negative. Of 1140 summer samples, $572(50.2 \%)$ were positive, 207 (18.2\%) positive with contaminants, $343(30.1 \%)$ overgrown and $18(1.6 \%)$ were negative. Mycobacteria were identified from $81.1 \%$ sites sampled in winter and $39.5 \%$ sites in summer. There were seasonal differences in the species found. Species identified in human samples from Brisbane residents, also found in water include $M$. gordonae, $M$. kansasii, $M$. abscessus/chelonae, $M$. fortuitum complex, M. simiae, $M$. intracellulare, $M$. avium, $M$. flavescens, $M$. interjectum, M. lentiflavum, M. mucogenicum, M. szulgai, and M. terrae.

Conclusion Pathogenic and nonpathogenic NTM can be found in water distributions systems in Brisbane, an area with a relatively high prevalence of NTM disease.

References

1 Thomson R. EID 2010; 16: 1576-83.

2 Thomson R, Carter R, Gilpin C, Coulter C, Hargreaves M. Appl Environ Microbiol 2008; 74: 3094-8.

Support Gallipoli Medical Research Foundation, The Prince Charles Hospital Foundation.

Conflict of Interest No.

\section{TP-185}

COMPARISION OF DIFFERENT TYPES OF NASAL SAMPLING FOR THE DETECTION OF HUMAN RHINOVIRUS IN CHILDREN

DW COX ${ }^{1,2}$, S-K KHOO 2 , J BIZZINTINO ${ }^{2}$, W-M LEE ${ }^{3}$, P DAVIS $^{2}$, L WEEKE ${ }^{2}$, EN SCHULTZ2, GC GEELHOED ${ }^{2}$, JE GERN ${ }^{3}$, J GOLDBLATT ${ }^{2}$, PN LE SOUEF ${ }^{1,2}$, IA LAING ${ }^{2}$

${ }^{1}$ Respiratory Medicine, Princess Margaret Hospital for Children, ${ }^{2}$ School of Paediatrics and Child Health, University of Western Australia, WA, Australia, and ${ }^{3}$ Department of Paediatrics, University of Wisconsin-Madison, Madison, WI, USA

CC DOBLER
${ }^{1}$ Woolcock Institute of Medical Research, The University of Sydney, NSW, and ${ }^{2}$ Department of Respiratory Medicine, Liverpool Hospital, Sydney, NSW

Aim To determine the incidence rate and nature of adverse events in patients taking treatment for latent tuberculosis infection (LTBI).

Methods Records of all patients who received treatment for LTBI at the Chest Clinic of a large tertiary hospital between 01/2000 and 04/2008 were reviewed. An adverse event was defined as any change in health status or side effect that led to treatment interruption or cessation. Liver function tests were not performed routinely during follow-up, except when the patient was considered to be at an increased risk of developing hepatitis.

Results Of 201 patients in whom treatment for LTBI was initiated $143(71 \%)$ received isoniazid for 6 months, $32(15 \%)$ received a combination of isoniazid and rifampicin for 6 months, and the remainder were treated with different regimens. Their mean (SD) age was 21 (17) years and $44 \%$ were male. Nineteen patients (9.5\%) experienced an adverse event. Seven patients developed a rash, four had lethargy and/or mood disorders, three had subclinical hepatitis, four experienced severe nausea, vomiting and/or other gastrointestinal symptoms and three had features of peripheral neuropathy. In eight patients who experienced an adverse event medication was temporarily ceased and then re-started without change; in four the treatment regimen was changed; and in seven the treatment was ceased completely. The risk of adverse events was not significantly related to age, sex, drug regimen (single drug versus combination therapy) or baseline transaminase levels.

Conclusions In this cohort almost 1 in 10 patients on treatment for LTBI experienced an adverse event. Although the adverse events were generally mild to moderate, this risk has to be taken into account when deciding whether to advise treatment for LTBI.

Supported by International Postgraduate Research Scholarship, University of Sydney.

Conflict of Interest No.
Introduction Human rhinovirus (HRV) is the commonest cause of asthma exacerbations in children. Pernasal aspirate (PNA) is the gold standard for microbiological sampling but is invasive and distressing for children. Studies have showed that less invasive swabs may be just as efficacious.

Aim To test the hypothesis that HRV detection is as efficient using nasal flocked swabs or washes and more comfortable, compared with PNA in children with respiratory illnesses. ment with respiratory symptoms. PNA was collected from one nostril of all children recruited and nasal flocked swab $(n=21)$ or wash $(n=12)$ collected from the other nostril alternately. Subjects rated the comfort of each sampling method 1 to 10 (least to most). Viral RNA was extracted and reverse transcribed. A two-step PCR of the HRV 5' UTR was used for detection, followed by sequencing for typing.

Results To date, 33 children ( $56 \%$ male, mean age of 3.32 years) had paired samples taken. Of these children, $67 \%(n=22)$ presented with a diagnosis of viral induced wheeze and $73 \%(n=24)$ had a HRV positive sample. Compared with PNAs, nasal flocked swabs were $85 \%$ (11 of 13 PNA positive) effective in detecting HRV, whilst nasal washes showed $100 \%$ (11 of 11 PNA positive) efficacy. Of the 25 successfully typed samples, 3 had HRVA and 22 had HRVC. Nasal washes had a better comfort rating (mean 9.09, $n=11$ ) than flocked swabs (mean 6.15, $\mathrm{n}=13$ ) and PNAs (mean 3.21, $\mathrm{n}=24$ ).

Conclusion Our findings suggest that whilst nasal flocked swabs are an effective sampling method for HRV detection, nasal washes were more effective, being as effective as PNAs and were the most comfortable.

Support NHMRC, PMH Foundation.

Nomination Nil.

Conflict of Interest No.

Journal Compilation (C) 2011 Asian Pacific Society of Respirology
Methods Children were recruited on presentation to the emergency depart- 
WITHDRAWN
DIFFERENTIAL IMPACT OF PANDEMIC H1N1 INFLUENZA ON REMOTE AND INDIGENOUS GROUPS OF THE NORTHERN TERRITORY: CROSS-SECTIONAL SEROLOGICAL STUDIES

JM TRAUER ${ }^{1}, \mathrm{KL}$ LAURIE $^{2}$, J MCDONNELL ${ }^{3}$, A KELSO $^{2}$, PG MARKEY $^{1}$ ${ }^{1}$ Centre for Disease Control, NT, Australia, ${ }^{2}$ WHO Collaborating Centre for Reference and Research in Influenza, Vic., Australia, and ${ }^{3}$ Menzies School for Health Research, NT, Australia

Aim To gauge the full impact of pandemic H1N1 influenza across demographic groups in the Northern Territory, particularly Indigenous and remoteliving individuals.

Methods We performed two cross-sectional serological surveys on specimens from residents of the Northern Territory, with 445 specimens obtained from January to May 2009 (pre-pandemic) and 1689 specimens from September 2009 (post-pandemic). Specimens were selected from among serum tubes collected from ambulatory outpatients. Antibody titres were measured by haemagglutination inhibition against the A/California/7/2009 reference virus. All specimens had available data for gender, age, and address, with Indigenous status determined in $94.1 \%$ of cases.

Results Protective antibody levels, defined as a titre of 40 or greater, were present in $7.6 \%$ of pre-pandemic specimens and $19.5 \%$ of post-pandemic specimens. The pre-pandemic proportion immune was greater with increasing age, but did not differ by other demographic characteristics. The post-pandemic proportion immune was greater among Aboriginal and Torres Strait Islanders and in younger age groups, but did not differ by gender or SocioEconomic Index For Area. However, the proportion immune was geographically heterogeneous, particularly among remote-living and Indigenous groups. The Northern Territory-wide attack rate adjusted to 2009 age, region and Indigenous status was $14.9 \%$.

Conclusions Pandemic influenza disproportionately affected children and Indigenous Australians in the Northern Territory in 2009. The proportion of specimens demonstrating post-pandemic immunity was particularly variable among Indigenous and remote-living individuals.

Conflict of Interest No.
TP-187

CARRIAGE OF HUMAN RHINOVIRUS (HRV) A WAS MORE COMMON THAN HRVC, IN ASYMPTOMATIC ABORIGINAL AND NON-ABORIGINAL CHILDREN FOLLOWED FROM BIRTH TO 2 YEARS OF AGE

A ANNAMALAY ${ }^{1}$, S-K KHOO ${ }^{1}$, J BIZZINTINO ${ }^{1}$, EN SCHULTZ ${ }^{1}$, G CHIDLOW ${ }^{2}$, W-M LEE ${ }^{3}, \mathrm{PJACOBY}^{4}, \mathrm{HC} \mathrm{MOORE}^{4}$, GB HARNETT ${ }^{2}$, DW SMITH ${ }^{2}$, JE GERN ${ }^{3}$, J GOLDBLATT ${ }^{1}$, D LEHMANN ${ }^{4}$, PN LE SOUEF ${ }^{1}$, IA LAING $^{1,4}$ AND THE KALGOORLIE OTITIS MEDIA RESEARCH PROJECT (KORMP) TEAM ${ }^{1}$ School of Paeds \& Child Health, Uni of WA, ${ }^{2}$ Pathwest Laboratories, ${ }^{3}$ Dept of Paeds, University of Wisconsin-Madison, Madison, WI, USA, and

${ }^{4}$ Telethon Institute for Child Health Research

The KORMP found asymptomatic Aboriginal children (AC) had more HRV than asymptomatic non-Aboriginal children (non-AC) in a longitudinal communitybased cohort study where infants had nasopharyngeal aspirates (NPA) collected regularly from birth to 2 years of age.

Aim To compare the frequency of HRV groups in asymptomatic AC and non-AC in the KORMP.

Methods NPA positive for HRV $(n=191)$ from the 1011 NPA previously tested for respiratory viruses, had viral RNA extracted and reverse transcribed. HRV was detected and typed using a two-step PCR of the HRV 5' UTR, followed by DNA sequencing for typing. Chi-square analyses were used.

Results HRV was detected and typed in 164 NPA (from 106 children; 55 AC and 51 non-AC), 16 could not be typed and 11 were not positive for HRV. AC had more HRV in summer and autumn than non- $A C$ and were more likely to be co-infected with at least $1 / 3$ bacterial species identified. HRVA, B \& C were found in $51.8,15.9$ and $32.3 \%$ of HRV detected. HRVB \& C were increased in infants exposed than not exposed to tobacco smoke in utero (HRVB; 4.3 vs. $2.0 \%, p=0.04$ and HRVC; 7.4 vs. $4.3 \%, p=0.046$ ). Of the $1011 \mathrm{NPA}$, HRV-A was detected more often in NPA from AC than non-AC (11.1 vs. $6.1 \%$, $p=0.006)$, particularly at $3-4$ months of age $(p=0.008)$ and during summer $(p<0.05)$. HRVB was detected more often in NPA from AC than non-AC in autumn $(p<0.05)$. HRVC was detected as often in AC as non-AC in each season except summer.

Conclusion The pattern of HRV group carriage varies with exposure to tobacco smoke, ethnicity, age and season.

Support NHMRC

Nomination Nil.

Conflict of Interest Nil.

\section{TP-189}

FEASABILITY OF LATENT TUBERCULOSIS INFECTION DIAGNOSIS BY IGRA, REMOTE FROM TESTING FACILITIES/319TRAUERJ IFEASIBILITY OF LATENT TUBERCULOSIS INFECTION DIAGNOSIS BY IGRA, REMOTE FROM TESTING FACILITIES

JM TRAUER ${ }^{1}, \mathrm{~K}^{\prime}$ HAJKOWICZ ${ }^{2}$, K FREEMAN ${ }^{3}$, VL KRAUSE ${ }^{1}$

${ }^{1}$ Tuberculosis Unit, Centre for Disease Control, NT, Australia, ${ }^{2}$ Department of Infectious Diseases, Royal Darwin Hospital, NT, Australia, and

${ }^{3}$ Department of Pathology, Royal Darwin Hospital, NT, Australia

Aim To determine whether interferon-gamma release assay (IGRA) can be effectively used for diagnosis of latent tuberculosis infection in a remote location.

Methods Subjects were enrolled from the Darwin Centre for Disease Control Tuberculosis clinic and were eligible if a tuberculin skin test (TST) of $10 \mathrm{~mm}$ or greater had been recorded for any indication. IGRAs were performed using Quantiferon®-TB Gold Whole Blood In-Tube assay according to manufacturer's instructions. Specimens were incubated and centrifuged at the local laboratory before refrigeration for transport. Interferon assay was performed at the reference laboratory, over $3000 \mathrm{~km}$ away.

Results 62 IGRAs were performed, with 42 patients $(68 \%)$ recording negative results, $19(31 \%)$ positive and only one result $(2 \%)$ indeterminate. Negative, and therefore discordant, test results were more common in BCG vaccinated individuals. This effect was not limited to those with TST results of $10-14 \mathrm{~mm}$, but was seen primarily in those with results of $15 \mathrm{~mm}$ and above. Conclusions These results are broadly comparable to findings for IGRA use in less remote settings. In particular, our low rate of indeterminate results suggests that IGRA testing is feasible at a remote site after local processing. This approach could be considered for use in the Northern Territory Tuberculosis Control Program.

Conflict of Interest No. 
RESPIRATORY NURSES SIG: POSTER SESSION

TP-190

\section{TACKLING NON-ADHERENT PATIENTS: A TREATMENT ADHERENCE TRAINING PROGRAM FOR CLINICIANS}

P MASEL ${ }^{1,2}$, C BOYLE $^{3}$, G POST $^{3,4}$, P FULBROOK $^{5,6}, \mathrm{~K} \mathrm{CLARK}^{7}$ ${ }^{1}$ Department of Thoracic Medicine, The Prince Charles Hospital, Qld, ${ }^{2}$ University of Queensland, Qld, ${ }^{3}$ Department of Mental Health, The Prince Charles Hospital, Qld, ${ }^{4}$ Australian Catholic University, Qld, ${ }^{5}$ Nursing Research \& Practice Development Centre. The Prince Charles Hospital, Qld, ${ }^{6}$ Australian Catholic University, Qld, ${ }^{7}$ Clinical Education and Training, Qld

Aim Informing a patient that they are at risk of developing a disease [such as COPD in a smoker] is rarely sufficient to change behaviour. Motivational Interviewing [MI] enhances clinician's effectiveness by initiating and supporting change. The aim of this training was to determine whether a treatment adherence program including MI techniques improved clinicians' knowledge of treatment adherence including the impact of non adherence, causative factors and management strategies.

Methods Participants were enrolled in a full day education program outlining strategies employed in Ml for health professionals. Validated questionnaires were administered prior to, immediately after and one month following the program.

Results Clinicians had increased awareness of the effectiveness of MI and stated stronger intentions to engage with their patients about their treatments and to explore adherence issues. Participants' knowledge was enhanced with the greatest development occurring in awareness of the impact of non-adherence to treatment strategies.

Conclusions The adherence training program did positively influence clinicians' knowledge of and attitude to adherence issues. Currently, further research will determine the efficacy of training on patient outcomes. Grant Support Office of the Chief Nursing Officer, Queensland. Conflict of Interest No.

\section{TP-191}

\section{FEEDBACK FROM CONSUMERS BRINGS COPD MODEL OF} CARE BACK TO BASICS

M Young ${ }^{1,5}$, M Brown $^{2}$, M Brooksbank ${ }^{3}$, G Crawford $^{3,4}$, T Burgess $^{3}$, A Crockett ${ }^{3}$, K Hancock ${ }^{3}$, R Antic ${ }^{5}$, D Kralik ${ }^{6}$, J Taylor ${ }^{7}$

${ }^{1}$ Transitional \& Community Service, ${ }^{2}$ The University of South Australia Adelaide, SA 5000, ${ }^{3}$ The University of Adelaide, Adelaide, SA, 5005, ${ }^{4}$ The Mary Potter Hospice, North Adelaide, SA,5006, ${ }^{5}$ Thoracic Medicine, The Royal Adelaide Hospital, Adelaide, SA, 5000, ${ }^{6}$ The Royal District Nursing Service, Wayville, SA 5034, and ${ }^{7}$ The Palliative Care Council of SA Eastwood, SA 5063

Introduction: The Adelaide Health Service is in the process of developing a new and innovative model of COPD community based care. A number of initiatives have informed this development including a recent research project examining the experiences of 15 participants with end stage COPD and their carers. A growing body of evidence indicates the importance of a palliative approach, however this often takes the form of referral to a palliative care service rather than a broader application of palliative principles in both specialist and primary care.

Methods: Fifteen participants were interviewed twice at 6 monthly intervals to explore their needs and the services they accessed. A series of focus groups with key service providers in SA was also undertaken. Data were analysed to identify how hospital, specialist palliative care units and primary care services currently interface to meet identified patient and carer needs.

Results: The current service model is episodic and reactive with services activated through the acute care system. Our research has shown that, as COPD advances, current models of care do not address the importance of supporting quality of life (including a focus on ADLs) and carers in their ongoing role. Also emphasised was the lack of co-ordination of care, collaboration between service providers and communication - the basics of chronic disease management.

Conclusions The outcomes of this study will inform the development of a proactive, multidisciplinary model of care which is no longer reliant on tertiary care, but places primary care at the centre of the model. Greater collaboration between respiratory, palliative and primary care services will provide an integrated approach, focusing on the needs of the patient and carer.

Supported by: NHMRC.

Nomination: Nil.

Conflict of interest No.

\section{AUDIT OF A WARD BASED NIV SERVICE IN A TERTIARY} HOSPITAL

\author{
JE HISLOP ${ }^{1}$, K PARKER ${ }^{1}$, J TOGHIL ${ }^{1}$, B BORG ${ }^{1}$, MT NAUGHTON ${ }^{1,2}$, \\ A YOUNG ${ }^{1,2}$ \\ The Alfred Sleep Disorders and Ventilatory Service, Dept of Allergy \\ Immunology \& Respiratory Medicine ${ }^{1}$, The Alfred and Monash University ${ }^{2}$, \\ Victoria, Australia
}

Aim To describe the inpatients treated by a dedicated NIV service. Methods A retrospective audit of inpatients treated by the Alfred NIV service between 1 January 2009 and 30 June 2009. The definition of NIV included patients treated with CPAP and bilevel positive pressure ventilation.

Results 150 patients (age: $58 \pm 17$ years (mean \pm SD), gender: $60 \%$ male) were treated with NIV on 172 occasions (repeat admissions 11 patients). Commonest indications for NIV were OSA ( $n=49,28 \%)$, acute exacerbations (AE) of COPD ( $n=33,19 \%)$, acute cardiogenic pulmonary oedema (ACPO) $(\mathrm{n}=18,10 \%)$ and post-lung transplantation $(\mathrm{n}=16,9 \%)$. Treatment was delivered primarily in the respiratory ward $(n=37,21 \%)$, cardiac ward $(n=19$, $11 \%)$, ICU $(n=16,9 \%)$ and general medical ward $(n=15,9 \%) .65$ episodes of CPAP (mean pressure $10 \pm 3 \mathrm{cmH} 2 \mathrm{O}$ ), OSA and ACPO made up $76 \%$ of those treated. Seventy-two episodes of bilevel PAP (mean IPAP $13 \pm 3 \mathrm{cmH} 2 \mathrm{O}$ and EPAP $6 \pm 1 \mathrm{cmH} 2 \mathrm{O}$ ), AECOPD and weaning post-mechanical ventilation made up $43 \%$ of those treated. Outcome data was available in a subgroup of patients with ACPO $(n=17)$ andAECOPD $(n=33)$. In the ACPO group, 9 patients $(53 \%)$ improved and NIV was ceased. Three patients $(18 \%)$ deteriorated and were intubated and 4 patients $(23 \%)$ were palliated. In the AECOPD group, 23 patients $(70 \%)$ improved andNIV was ceased or they were discharged on therapy. 10 patients either deteriorated on NIV or could not tolerate therapy, of these $7(21 \%)$ continued ward management and $3(9 \%)$ were palliated.

Conclusion The Alfred NIV service model has managed a large number of referrals across a range of diseases in a variety of wards. This is likely to have reduced demand on ICU, HDU and respiratory ward beds. Compared to the published literature, theoutcomes for ACPO are worse than expected but comparable for AECOPD. This may be explained by local referral patterns for ACPO. We believe that our service model provides a viable means of administering NIV to an ever expanding referral base. Conflict of Interest No. 
CONFUSING OUR PATIENTS: PRESCRIPTION AND ADMINISTRATION OF INHALED MEDICATIONS ON MEDICAL WARDS

\section{J BINNERSLEY, JG SERGINSON}

Caboolture Hospital, QId, Australia

Inhaled medications form the mainstay of drug treatment for patients with airways disease. Effectiveness of therapy is dependent on the appropriate selection and prescription of drug and device, correct supply and adherence to therapy with an effective technique. Patients frequently admit to acute medical wards both with acute exacerbations and for other co-morbidities eg heart failure or pneumonia. Inpatient episodes provide an opportunity to review inhaled therapy however anecdotally add to patient confusion and introduce complexity (rational or ad hoc changes to inhaled drug, device, strength, dose or frequency).

Aim Identify prescribing accuracy and effectiveness of patients' inhaler technique. Describe any discrepancies between inhaled therapy: (1) used prior to admission, (2) prescribed for inpatient use, (3) available at the bedside and (4) administered, prior to and after implementation of an inhaler prescribing and administration guide.

Methods A single day audit of all inpatients on 2 general medical wards was conducted October 2010 (review of medication charts and inhalers in patients' bedside lockers, brief questioning and direct observation of patients' inhaler technique. Results compared to post implement of the 'Prescribing and Administering Inhalers' tool (audit in December 2010).

Results 19 from 58 (33\%) patients had inhalers prescribed, (mean: 2.4 prescriptions per patient). $95 \%$ of prescriptions were accurate ( $88 \%$ patient had no errors). Discrepancies between used prior to admission and inpatient prescriptions were found in $7(41 \%)$ patients while those between inpatient prescriptions and available at the bedside were found in $29 \%$. Self-administration ('S') was noted on medication charts of $79(53 \%)$ patients, 3 of whom had an ineffective inhaler technique. $2 / 17$ patients has a spacer at the bedside with a further $r 5$ prescribed metered aerosol inhalers. Post-intervention differences in prescribing, supply, administration and technique errors will be discussed.

Conclusions A combination of errors and prescription discrepancies reduce the effectiveness of inhaled therapy for inpatients.

Conflict of Interest No. 